\title{
ANALYSIS OF MEASUREMENTS OF DEFECTS IN MULTIAXIAL WARP KNITTED FABRICS FOR CFRP COMPOSITES
}

By

Diane Suk-Ching Liu

B.Eng. Ryerson University, 2008

\author{
A thesis \\ Presented to Ryerson University
}

In partial fulfillment of the

Requirements for the degree of

Master of Applied Science

in the Program of

Mechanical Engineering

Toronto, Ontario, Canada, 2011

CDiane Suk-Ching Liu 2011 


\section{AUTHOR'S DECLARATION}

I hereby declare that I am the sole author of this thesis or dissertation.

I authorise Ryerson University to lend this thesis or dissertation to other institutions or individuals for the purpose of scholarly research.

Diane Suk-Ching Liu

Toronto, Ontario, Canada

May 11, 2011

I further authorise Ryerson University to reproduce this thesis or dissertation by photocopying or by other means, in total or part, at the request of other institutions or individuals for the purpose of scholarly research.

Diane Suk-Ching Liu

Toronto, Ontario, Canada

May 11, 2011 


\section{BORROWER}

Ryerson University requires the signatures of all persons using or photocopying this thesis.

Please sign below and give address and date. 


\section{ABSTRACT}

Analysis of Measurements of Defects in Multiaxial Warp Knitted Fabrics for CFRP Composites Diane Suk-Ching Liu

Master of Applied Science

2011

Mechanical Engineering

Ryerson University

Multiaxial Warp Knitted (MWK) Fabrics are used to create Carbon Fibre Reinforced Plastic (CFRP) laminates. In contrast to Prepregs, CFRP laminates made with MWK fabrics are of interest because they could lower costs and processing time by being already constructed with multiple layers and through the use of a hot air oven instead of an autoclave. Defect in the form of fibre angle orientation plays an important role in the compression strength of a laminate, where compression strength has been found to be lower than tensile strength for laminates made with MWK fabrics. The in-plane and out-of-plane waviness of the fibres were characterised by the standard deviation of the angular waviness: sample Standard deviation of Fibre In-plane (SFI) and the sample Standard deviation of Fibre Out-of-plane (SFO). The SFI value was found in two ways: analySIS (Multiple Field Image Analysis (MFIA) technique) software and Fibre Image Analysis software. Measurements of the holes in the carbon fibre textile, colloquially known as "fisheyes," caused by sewing the textile together were also gathered. The SFI, SFO, and "fisheye" dimensions were together used in the FMB-PMB model and the Unit Cell Model to calculate the compression strength. These predicted compression strengths were compared to the laboratory results. Also, a reliability model was developed to find $R$, the reliability of each textile, to be used as a textile classification tool. It has been found that the compression strength predictions found using analySIS and Fibre Image Analysis yielded similar results, with predictions from analySIS closer to the laboratory results. The $R$ value yielded a positive correlation with the results from analySIS. A large percentage of difference between the predicted and the actual compression strength was observed for some textiles. This could be attributed to the inherent lack of regularity for some of the examined textiles and variability in determining the SFI and "fisheye" parameters. Improvements would involve devising rules and methods to determine the SFI and "fisheye" parameters, modifying the FMB-PMB and Unit Cell Models, and making the analysis process faster and more applicable for on-line quality process control. 


\section{ACKNOWLEDGEMENTS}

This thesis would not have been possible without those who supported and assisted me through this journey.

Thanks to those at Ryerson University: Dr. Kouroush Jenab (supervisor), Dr. Greg Kawall (Director, Graduate Studies, Mechanical and Industrial Engineering), Dr. Liping Fang (Chair, Mechanical and Industrial Engineering), and the administrative staff at the Department of Mechanical and Industrial Engineering and at the School of Graduate Studies. Also, thanks to my oral examining committee members: Dr. Hua Lu and Dr. Habiba Bougherara for reviewing my thesis.

Special thanks to the people who are and were in Bremen, Germany: Dipl.-Ing. Julian Kuntz (project supervisor; Premium AEROTEC GmbH), Dipl.-Ing. Mathias Wietgrefe (project co-supervisor; DLR), Dr.-Ing. Klaus Edelmann (Airbus Operations GmbH) on "Fisheyes," Stefan Schwaneberg on MFIA and "Fisheyes," Dr. Andrea Miene on Fibre Image Analysis, Dipl.-Ing. Steffen Pangerl for scientific support, Norbert Czelk for technical support in the laboratory, Chinh Nguyen and Romain Cucheval for assistance in the SFO laboratory results, Umut Polat for assistance with German to English translations, Benjamin Saage for assistance with Fibre Image Analysis, and the administrative staff at Airbus Operations GmbH. Also thanks to Monika Bannas, former landlady, for her emotional support during my stay in Germany.

Thanks to my family: Amy Liu (mother), David Lau (brother), and Winston Lau (brother), for their emotional support on a journey that has witnessed the difference of two continents and the passing of my father, Yick-Cheong Liu. 


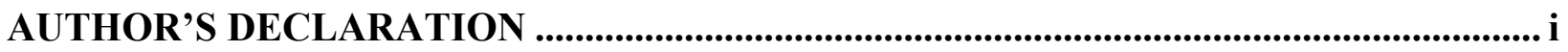

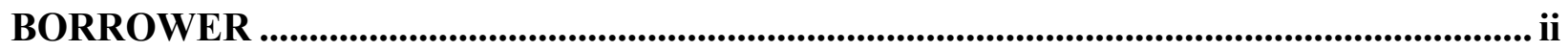

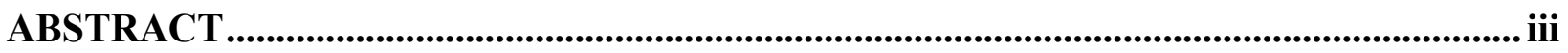

ACKNOWLEDGEMENTS ............................................................................................................. iv

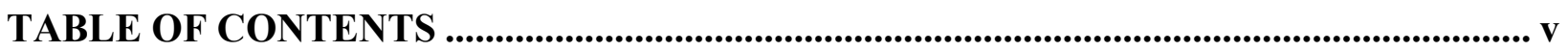

LIST OF TABLES ............................................................................................................................... vii

LIST OF FIGURES ............................................................................................................

LIST OF ABBREVIATIONS ........................................................................................................... xi

NOMENCLATURE............................................................................................................................. xiv

CHAPTER 1: INTRODUCTION............................................................................................. 1

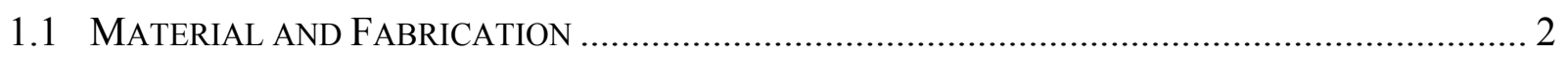

CHAPTER 2: LITERATURE REVIEW ON COMPOSITE DEFECT ................................. 8

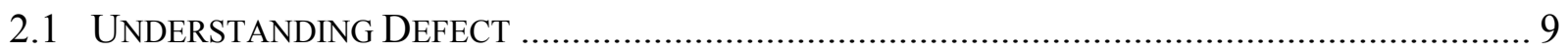

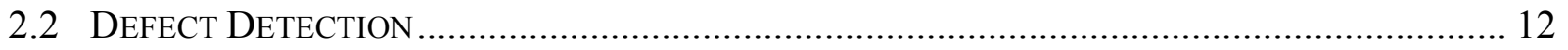

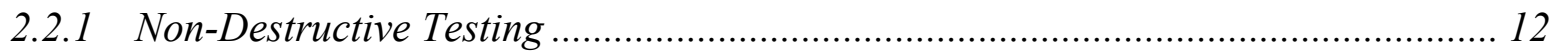

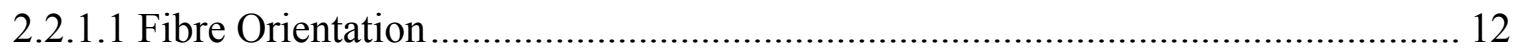

2.2.1.2 Fibre Orientation, "Fisheyes" and Other Defects ............................................. 24

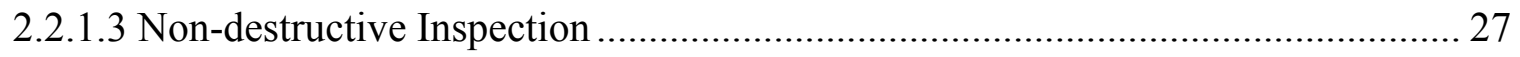

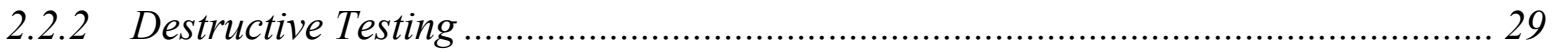

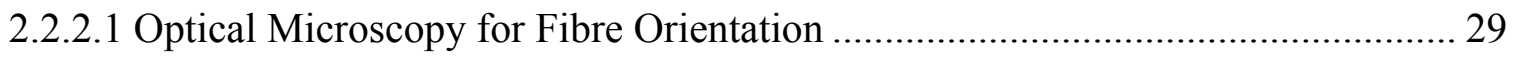

2.2.2.2 Compression and Tensile Testing ............................................................... 34

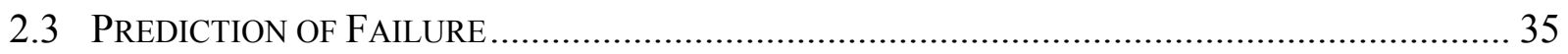

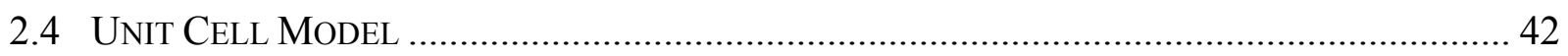

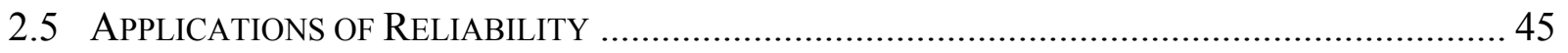

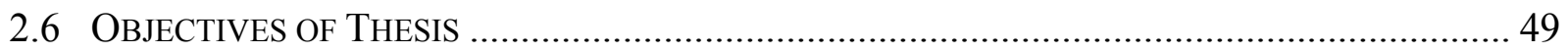

CHAPTER 3: ANALYSIS OF MEASUREMENTS OF COMPOSITE DEFECT................50

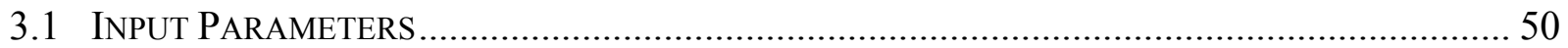

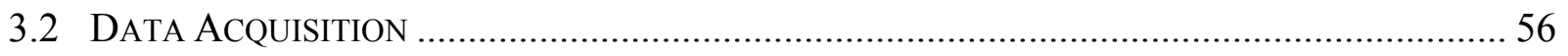

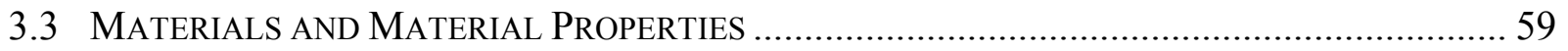

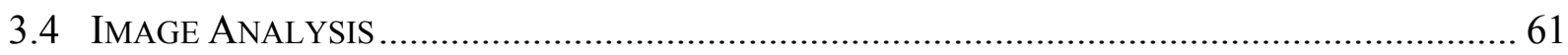

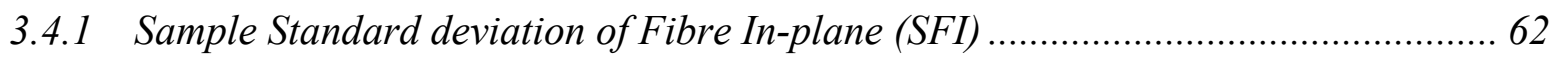

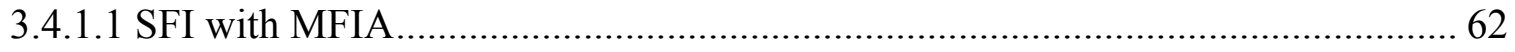

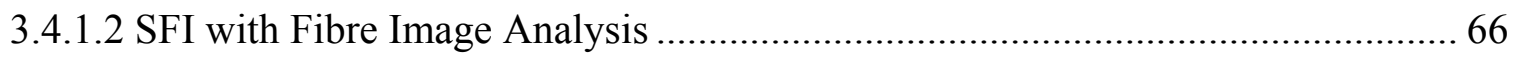


3.4.2 Fisheyes or Stitching Yarn Distortions ....................................................... 75

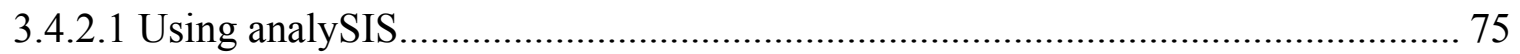

3.4.2.2 Using Fibre Image Analysis........................................................................ 84

3.4.2.3 Parametric Study with the Unit Cell Model.................................................... 85

3.5 Sample Standard Deviation of Fibre Out-OF-Plane (SFO) ................................... 87

3.5.1 Analysis of Collected Waviness Data ........................................................... 88

CHAPTER 4: RELIABILITY MODEL FOR COMPOSITES .............................................. 93

4.1 Probability Density Function (PDF) FitTING......................................................... 93

4.2 Compression Strength of A Ply Based on ContinuUm Damage Model.................. 95

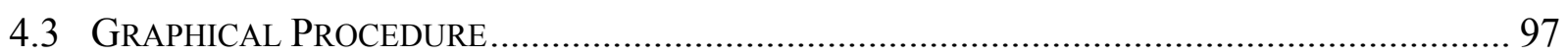

4.4 Proposed Numerical Procedure, Applied to Current Problem............................ 98

4.5 AN EXAMPLE USING THE RELIABILITY MODEL ....................................................... 101

CHAPTER 5: RESULTS AND CONCLUSIONS ........................................................... 104

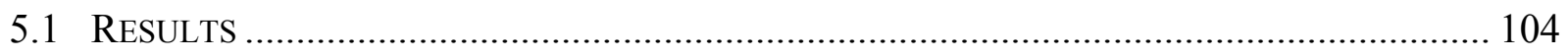

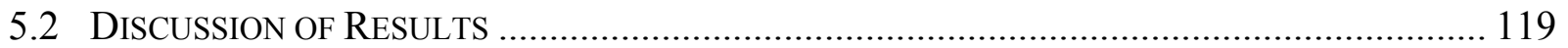

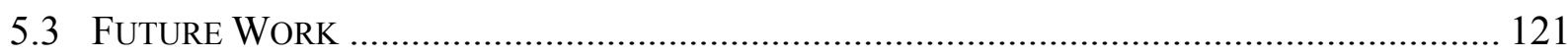

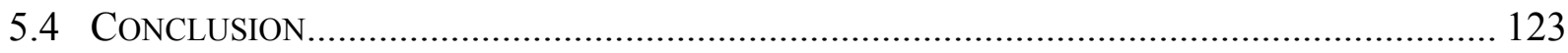

REFERENCES ...................................................................................................................................... 124

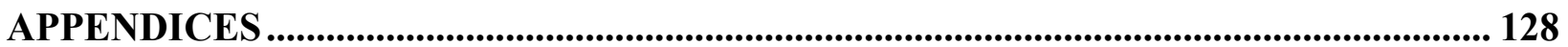

A. FORMULA FOR THE SAMPLE STANDARD DEVIATION OF TWO

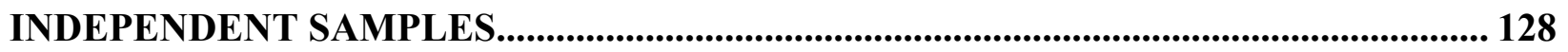

B. MODIFIED FORMULAS FOR THE SAMPLE STANDARD DEVIATION OF TWO

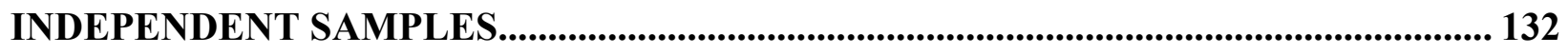

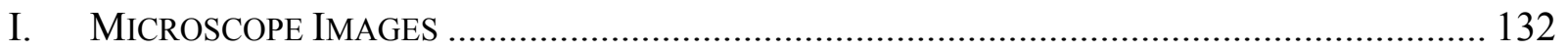

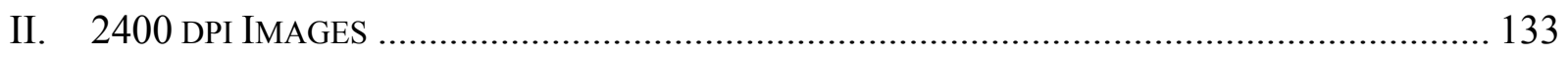

C. NORMALISATION USING CLASSICAL LAMINATION THEORY .................... 135 


\section{LIST OF TABLES}

Table 1: Defects and Appropriate NDI Methods (Armstrong \& Barrett, 1998) .......................... 28

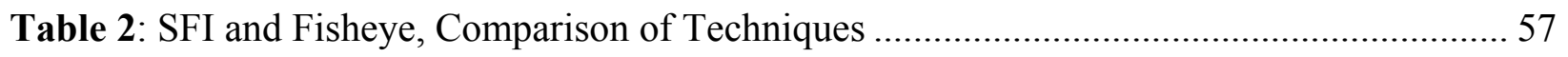

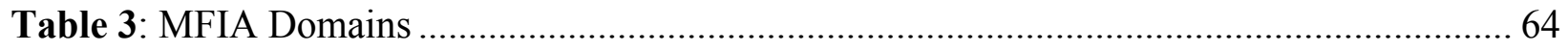

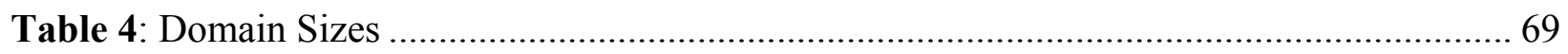

Table 5: Comparing SFI found from MFIA and Fibre Image Analysis (textile: 11-2 (DS))...... 69

Table 6: Image Size and Calculation Time with Fibre Image Analysis with 2038 MB RAM

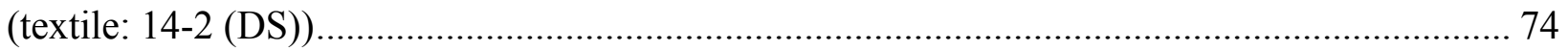

Table 7: Image Size and Calculation Time with Fibre Image Analysis with 1014 MB RAM

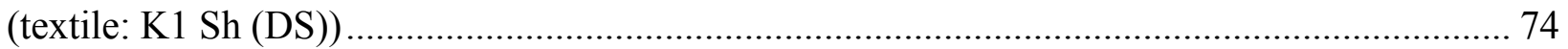

Table 8: Image Size and Calculation Time with Fibre Image Analysis with 4096 MB RAM

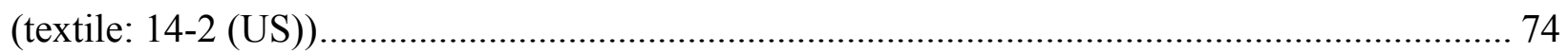

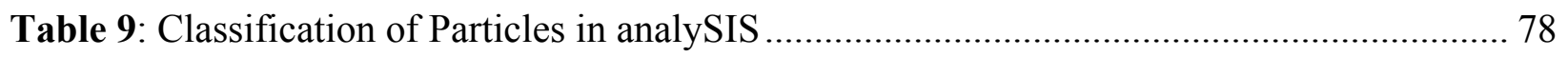

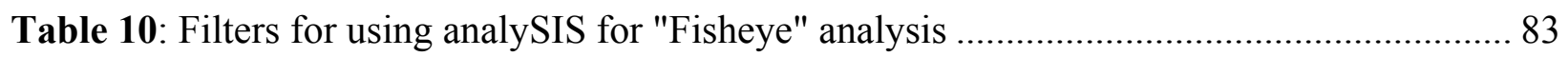

Table 11: Hypothetical Compressing Testing Results ............................................................. 101

Table 12: $P$-values for Fitted PDF's for Hypothetical Compression Testing Results................ 101

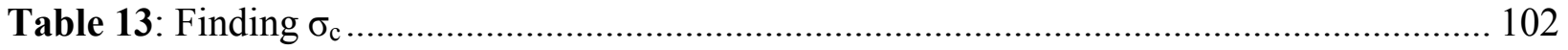

Table 14: $P$-values for Fitted PDF's for Results in "Column 5" of Table 13 ……..................... 103

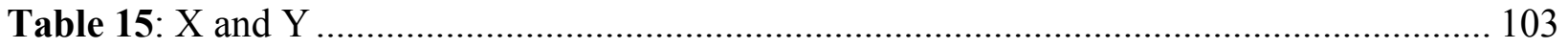

Table 16: (Normalised and Corrected, with Correction Factor $=2.6413045648049$ ) Predicted Compression Results from analySIS and Fibre Image Analysis, Compared to Laboratory Results

108

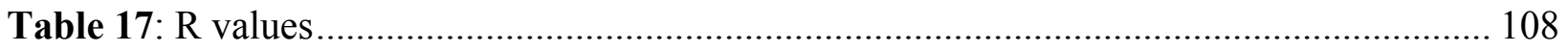

Table 18: Percent Difference between Actual Results............................................................. 109

Table 19: Anova: Two-Factor Without Replication (*11-1 Sh (DS) data has been removed from ANOVA analysis)....

Table 20: Correlation between R value, SFI, SFO, and Compression Strength Values, Data from

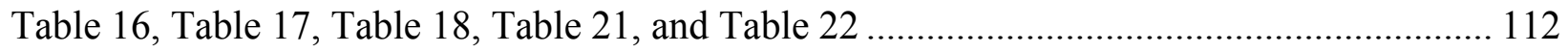

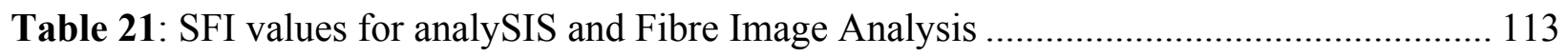

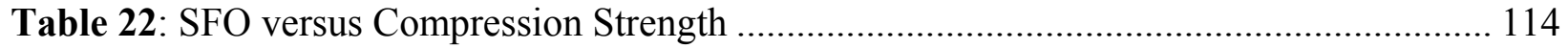

Table 23: Fisheye Width and Length Versus Compression Strength from analySIS ................ 115

Table 24: Fisheye Width and Length versus Compression Strength from Fibre Image Analysis

Table 25: Correlation between $\mathrm{R}$ value, Compression Strength, Length, and Width of Fisheyes found with analySIS and Fibre Image Analysis, Data from Table 17, Table 23, and Table 24. 117 Table 26: Comparison between Percent Differene and Standard Deviation between Actual Compression Testing Results 118

Table 27: Correlation between Percentage Difference and Standard Deviation of Actual Results 


\section{LIST OF FIGURES}

Figure 1: Usage of composite materials in various components of the Boeing 777 aircraft

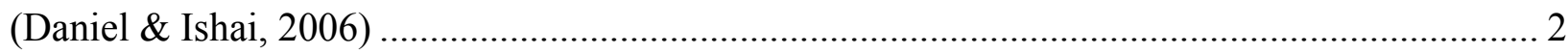

Figure 2: Classification of composite material systems (Daniel \& Ishai, 2006) ........................... 3 Figure 3: 2D orthogonal weave fabric (plain weave), 3D orthogonal woven fabric (Miller, 1996)

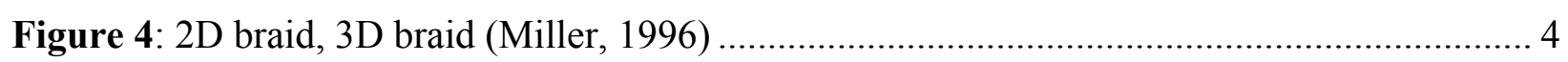

Figure 5: Knitted fabric, Warp (Tricot) Knit (Miller, 1996).................................................... 4

Figure 6: Schematic of multiaxial warp knitting processing (Hörsting, Wulhorst, Franzke, \&

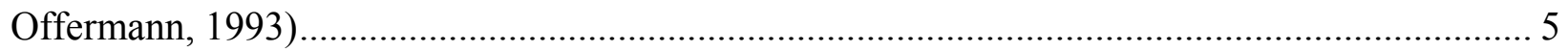

Figure 7: Rosen's Fibre Micro-buckling Model (Schultheisz \& Waas, 1996) ............................ 10

Figure 8: Geometry of kink bands (Schultheisz \& Waas, 1996) ............................................... 11

Figure 9: I-randomness Procedure for Discontinuous Fibres (Pourdeyhimi et al., 1996a) ......... 17

Figure 10: $\mu$-randomness Technique for Continuous Fibres (Pourdeyhimi et al., 1996a).......... 18

Figure 11: Square Raster Direction Coding (Pourdeyhimi et al. , 1996b) ................................... 19

Figure 12: Line Representation and Corresponding Hough Transform (Pourdeyhimi et al., 2002)

Figure 13: Schematic of the Directional Lighting System Used to Capture Nonwoven Images (Pourdeyhimi et al., 1999)

Figure 14: Edge Thresholded Image (Pourdeyhimi, Dent, Jerbi, Tanaka, \& Deshpande, 1999) 23

Figure 15: Thinning and Pruning Examples: (a) Top: Ideal Image with Minimal Branching, (b) Middle: Noisy Image Resulting in Excessive Branching, (c) Bottom: Noisy Image Filtered to Minimize Branching (Pourdeyhimi et al., 1999) …………...................................................... 23

Figure 16: NCF with Exemplary Defects (Schneider et al., 2004) ......................................... 24

Figure 17: Detailed Picture of NCF (Schneider et al., 2004) ……………................................ 25

Figure 18: Detail of Analysed NCF (Schneider et al., 2004) ................................................... 26

Figure 19: Summary of Final Results (Schneider et al., 2004) ................................................ 26

Figure 20: Ellipse resulting from the plane section of a right circular cylinder (left); Definition

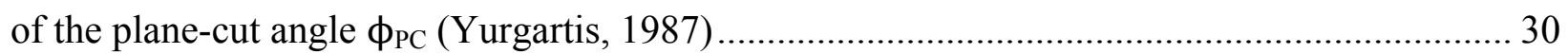

Figure 21: Definition of the in-plane and out-of-plane angles, $\varnothing$ and $\theta$, respectively. The fibre

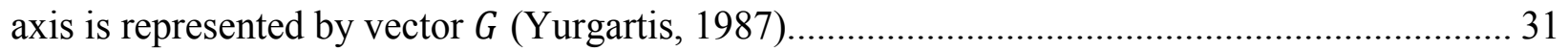

Figure 22: Optical micrograph of a pultruded composite, section cut in parallel to pultrusion direction, with a domain and pixel array highlighted (left); Schematic depiction of domains $\left(A_{j}\right.$ centred at $\mathrm{x}_{\mathrm{j}}, \mathrm{y}_{\mathrm{j}}$ ) within which the average fibre orientation $\varnothing$ is to be measured (right) (Creighton et al., 2001) 33

Figure 23: Schematic depiction of a 1D array of pixels within an image, inclined at angle $\theta$ to the reference direction, showing typical intensity distributions along the length of the array, for two values of $\theta$, one which is equal to and the other greater than the fibre misalignment angle $\varnothing$ (Creighton et al., 2001) 33 
Figure 24: a) Optical micrograph showing the free surface of prepregged sheet material, with the centroid of each measurement domain marked by a black dot, b) Corresponding contour map of measured average misalignment angle within each domain, with each grid intersection point corresponding to one of the black dots on the micrograph (Creighton et al., 2001) .................. 33

Figure 25: IITRI compression test fixture (Mallick, 1997) ................................................ 34

Figure 26: Hierarchical structure of the FMB-PMB approach (Pansart, 2008) ........................ 36

Figure 27: Idealization of the composites's microstructure and FMB FE model geometry

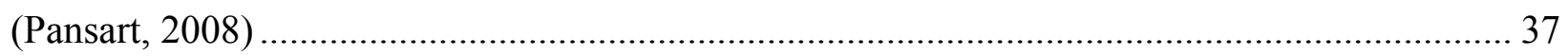

Figure 28: Loads of FMB (Pansart, 2008) ........................................................................... 38

Figure 29: Finite element ply meso-buckling model (Pansart, 2008) .................................... 40

Figure 30: Addition of two angles in perpendicular planes (Pansart, 2008)............................ 40

Figure 31:(a, b) NCF manufacturing process, (c, d) structural stitching of [A1-B-A2] NCF layup [21-23]. (a) NCF production, (b) example of finished NCF, (c) through-thickness stitching to insert structural yarn, (d) structurally stitched laminate of NCF layers (tension laminate; modified lock stitch) (Heß et al., 2007) ........................................................................ 42

Figure 32: NCF lay-up of tension and compression CFRP laminates (Heß et al., 2007)........... 43

Figure 33: Micrograph through a $-45^{\circ}$ layer of a structurally stitched [A1-B-A2] CFRP laminate parallel to laminate plane (Heß et al., 2007) ....................................................................... 43

Figure 34: Unit-cell model of stitched [A1-B-A2] CFRP tension laminate (Heß et al., 2007) ... 44 Figure 35: Structural stitching of NCF laminates: stitch patterns (spacing s and pitch length $p$ ) and stitch density SD (Heß et al., 2007) ...................................................................... 44

Figure 36: Probability distribution functions for breaking and operating loads (left), Additional safety factors as functions of the coefficient of variation. $f_{b}=1.3$ (1) and 1.5 (2) (right) (Rabotnov

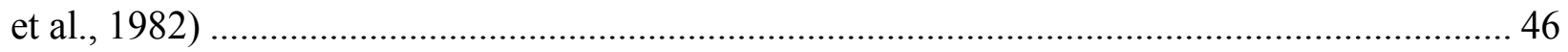

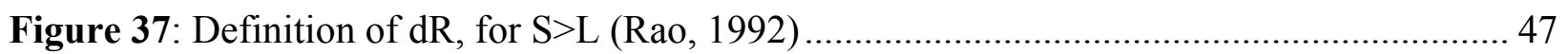

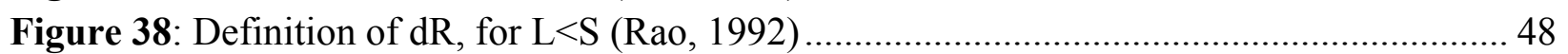

Figure 39: Interference Area, Probability of Failure (Rao, 1992) ........................................ 48

Figure 40: FMB and PMB Calculations for Prediction of Compression Strength .................... 52

Figure 41: Determination of Compression Strength of Overall System (Rehbein, 2009) .......... 56

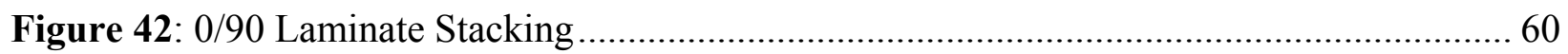

Figure 43: Sheared 0/90 Laminate Stacking .................................................................... 61

Figure 44: "Trial" in MFIA clamping device, a) without lid b) with lid ................................ 63

Figure 45: MFIA Microscope Image with Domains ........................................................... 63

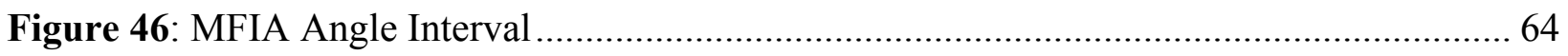

Figure 47: Analysed Microscope Image using Fibre Image Analysis, SFI versus Domain Size

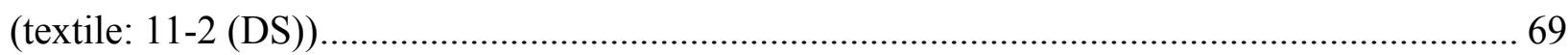

Figure 48: Fibre Image Analysis, Result File 2 .......................................................... 71

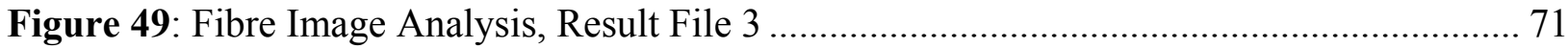

Figure 50: Fibre Image Analysis, Result File 1 ............................................................ 72

Figure 51: Fibre Image Analysis, Result File 4 .......................................................... 73

Figure 52: Phase Analysis using AnalySIS, Brightness Histogram...................................... 76 
Figure 53: Derivation Graph, Derivation of Brightness Histogram......................................... 77

Figure 54: Extension Interior Min (Ausdehnung Innen Min) ............................................... 77

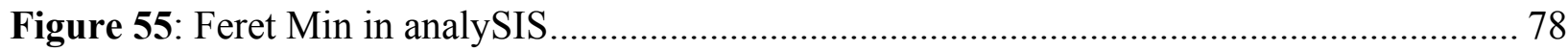

Figure 56: Calculation of Average Length of "Fisheye" ................................................... 79

Figure 57: Textile 11, Clear Identifiable "Fisheyes" a) Original Textile b) Original Textile after Particle Analysis c) Sheared Original Textile d) Sheared Original Textile after Particle Analysis

Figure 58: Textile KMV, Not Clear Identifiable "Fisheyes" a) Original Textile b) Original Textile after Particle Analysis c) Sheared Original Textile d) Sheared Original Textile after Particle Analysis .................................................................................................... 81

Figure 59: Feret Min - Extension Interior Min (Ausdehnung Innen Min)............................... 82

Figure 60: Parameters of Unit Cell Model (Rehbein, 2009) ................................................. 86

Figure 61: Measurement of Waviness using a Perthometer manufactured by Mahr................. 88

Figure 62: Measurement of SFO (Up Side, Down Side) ..................................................... 88

Figure 63: Equilibrium Stress for Various Fibre Misalignment Angles $(\alpha)$ (Barbero, Prediction of compression strength of unidirectional polymer matrix composites, 1998) ......................... 96

Figure 64: Imperfection Sensitivity (Barbero, Prediction of compression strength of

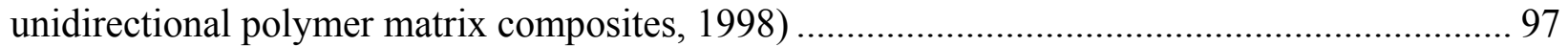

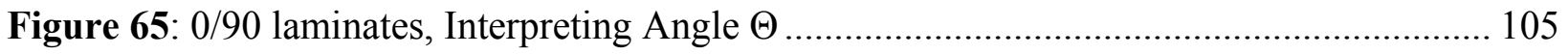

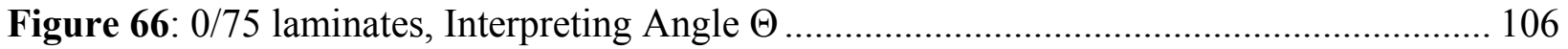

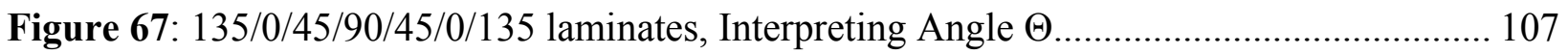

Figure 68: (Normalised and Corrected) Predicted Compression Strength vs. Textile, Plot of Table 16 and Table 18.................................................................................................... 110

Figure 69: Plot of Table 21, Predicted Compression Strength versus SFI .............................. 113

Figure 70: Plot of Table 22, Predicted Compression Strength versus SFO ............................ 114

Figure 71: Scatter of Data from Table 23 and Table 24, Compression Strength versus Length of "Fisheyes" 116

Figure 72: Scatter of Data from Table 23 and Table 24, Compression Strength versus Width of "Fisheyes". 116

Figure 73: Scatter of Data from Table 23 and Table 24, Length versus Width of "Fisheyes".. 117 


\section{LIST OF ABBREVIATIONS}

\begin{tabular}{|c|c|}
\hline ANOVA & Analysis of Variance \\
\hline CCD & Charged-couple Device \\
\hline $\mathrm{CDF}$ & Cumulative Distribution Function \\
\hline $\mathrm{CDF}$ & Cumulative Distribution Function \\
\hline CFRP & Carbon Fibre Reinforced Plastic \\
\hline CLT & Classical Lamination Theory \\
\hline CRT & Cathode-ray Tube \\
\hline DS & Down Side \\
\hline DT & Destructive Testing \\
\hline DT & Destructive Testing \\
\hline EPCM & Electric Potential Change Method \\
\hline ERCM & Electric Resistance Change Method \\
\hline $\mathrm{FE}$ & Finite Element \\
\hline FEM & Finite Element Model \\
\hline FEM & Finite Element Method/Model \\
\hline FFT & Fast Fourier Transform \\
\hline FIBRE & Faserinstitut Bremen \\
\hline FMB & Fibre Micro-Buckling \\
\hline FMB & Fibre Micro-Buckling Model \\
\hline FORM & First-order stochastic approximation \\
\hline FRP & Fibre Reinforced Plastic(s) \\
\hline $\mathrm{FVC}$ & Fibre Volume Content \\
\hline
\end{tabular}




\begin{tabular}{|c|c|}
\hline FVF & Fibre Volume Fraction, see FVC \\
\hline GUI & Graphical User Interface \\
\hline HS & High Strength Carbon Fibre \\
\hline IM & Intermediate Strength Carbon Fibre \\
\hline IVW & Institut für Verbundwerkstoffe \\
\hline $\mathrm{K}-\mathrm{S}$ & Kolmogorov-Smirknov test \\
\hline MFIA & Multiple Fibre Image Analysis \\
\hline MFIA & Multiple Field Image Analysis \\
\hline MWK & Multiaxial Warp Knitted Fabric(s) \\
\hline NCF & Non-Crimp Fabric \\
\hline NDI & Non-destructive Inspection \\
\hline NDT & Non-Destructive Testing \\
\hline NDT & Non-Destructive Testing \\
\hline ODF & Fibre Orientation Distribution \\
\hline PDF & Probability Density Function \\
\hline PDF & Probability Density Function \\
\hline PMB & Ply Meso-Buckling \\
\hline PMB & Ply Meso-buckling Model \\
\hline Prepreg & Pre-impregnated tape \\
\hline RAM & Random Access Memory \\
\hline $\mathrm{RC}$ & Reinforced Concrete \\
\hline RTM & Resin Transfer Moulding \\
\hline SFI & Standard Deviation of Fibre In-Plane \\
\hline SFI & Standard deviation of Fibre In-plane \\
\hline
\end{tabular}


SFO Standard Deviation of Fibre Out-of-Plane

SFO Standard deviation of Fibres Out-of-plane

SMC Sheet Moulding Compound

SMC Sheet Moulding Compound

UD Unidirectional Laminate

US Up Side

VBA Visual Basic for Applications 


\begin{tabular}{|c|c|}
\hline$\tilde{\epsilon}_{\text {crit }}$ & Ply Critical Strain, in PMB model (2.3) \\
\hline$\epsilon_{\text {crit }}$ & Critical Strain, in FMB model (2.3) \\
\hline$|\Delta \mathrm{G}|$ & Edge Magnitude (2.2.1.1) \\
\hline$\varnothing$ & Angle with respect with the $0^{\circ}$ Direction $(2.2 .2 .1)$ \\
\hline$\emptyset_{0}$ & Sum of Original Misorientation (2.2.2.1) \\
\hline$\emptyset_{p c}$ & Plane-cut Angle (2.2.2.1) \\
\hline$\Delta_{H}$ & Horizontal Difference (2.2.1.1) \\
\hline$\Delta_{V}$ & Vertical Difference (2.2.1.1) \\
\hline$\Delta \mathrm{x}$ & Gradient Operator in x Direction (2.2.1.1) \\
\hline$\Delta y$ & Gradient Operator in y Direction (2.2.1.1) \\
\hline$A$ & Source Image (2.2.1.1) \\
\hline$A_{0}$ & Amplitude of Fibre (2.3) \\
\hline$b$ & Average Width of "Fisheye" [mm] (3.4.2.3) \\
\hline$c_{R, 0}$ & Regression Coefficient (3.5) \\
\hline$c_{R, 1}$ & Regression Coefficient (3.5) \\
\hline$d$ & Length of Distance, $\mu$-randomness, Hough Transform (2.2.1.1) \\
\hline$E_{11, f}$ & Elastic Modulus of Fibre, In-fibre Direction (2.3) \\
\hline$E_{11}$ & Elastic Modulus of Laminate, In-fibre Direction (2.3) \\
\hline$E_{m}$ & Elastic Modulus of Matrix (2.3) \\
\hline$F$ & Maximum Number of Sampling Points (3.5) \\
\hline$f_{L}(l)$ & Probability Density Function (PDF) of Load (L) (2.5) \\
\hline$f_{S}(s)$ & Probability Density Function (PDF) of Strength (S) (2.5) \\
\hline$G_{12 c}$ & Shear Modulus of Composite (2.2.2.1) \\
\hline$I$ & Light Intensity (2.2.2.1) \\
\hline$\ell_{H}$ & Half Length of "Fisheyes" (3.4.2.1) \\
\hline \multirow[t]{2}{*}{$\ell$} & Length, I-randomness, Hough Transform (2.2.1.1) \\
\hline & Average Length of "Fisheye" [mm] (3.4.2.3) \\
\hline$L$ & Load (2.5) \\
\hline
\end{tabular}




\begin{tabular}{|c|c|}
\hline$\ell_{\mathrm{F}}$ & Average Length of "Fisheye" (3.4.2.1) \\
\hline$m_{a}$ & Areal Weight of Everything except the Carbon Fibre $\left[\mathrm{g} / \mathrm{m}^{2}\right]$ (3.3) \\
\hline$m_{l}$ & Areal Weight of the Layer $\left[\mathrm{g} / \mathrm{m}^{2}\right](3.3)$ \\
\hline$n$ & Number of Layers with the Same Areal Weight of the Layer (3.3) \\
\hline$N$ & Number of All Measured Points (3.5) \\
\hline$N_{a}$ & Number of Items in Trial A (3.4.1.1) \\
\hline$N_{b}$ & Number of Items in Trial B (3.4.1.1) \\
\hline$N_{t}$ & Total Number of Items in Trial A and B (3.4.1.1) \\
\hline$N_{\theta}(k)$ & Number of Points Satisfying $\frac{(2 k-1) \pi}{2 n} \leq \theta<\frac{2 k+1}{2 n}(2.2 .1 .1)$ \\
\hline$p$ & Stitch Length $[\mathrm{mm}]$ (3.4.2.3) \\
\hline$P_{0} b$ & Median of the Breaking Load (2.5) \\
\hline$P_{0} s$ & Median of the Operating Load (2.5) \\
\hline $\mathrm{R}$ & Reliability, Probability of Success (2.5 and 4) \\
\hline \multirow[t]{2}{*}{$r$} & Pearson Product-Moment Correlation Coefficient (5) \\
\hline & Perpendicular Distance from Origin, Hough Transform (2.2.1.1) \\
\hline$R(e)$ & $\begin{array}{l}\text { Placement Rule or Relation for element e, equal to texture } \mathrm{f} \\
(2.2 .1 .1)\end{array}$ \\
\hline$r_{s}$ & Spearman's Coefficient of Rank Correlation (2.2.1.1) \\
\hline$s$ & Stitch Distance $[\mathrm{mm}](3.4 .2 .3)$ \\
\hline \multirow[t]{2}{*}{$\beta$} & Probability of Failure over Specified Period of Service (2.5) \\
\hline & Angle of Kink Band (2.1) \\
\hline$t$ & Mean Measured Thickness of the Laminate [mm] (3.3) \\
\hline$u_{1, c r i t}^{r 1}$ & Critical Displacement of Node r1 (2.3) \\
\hline$u_{x, c r i t}^{r 3}$ & Critical Displacement of Node r3 (2.3) \\
\hline$u_{x, F M B}^{r 3}$ & Displacement which $\frac{\sigma_{11, e q}}{\widetilde{\sigma}_{11}^{c u, F M B}} \geq 1$ is reached for the First Time (2.3) \\
\hline$V_{f}$ & Fibre Volume Content (FVC), Three-dimensional (2.3) \\
\hline$V_{f}^{*}$ & Fibre Volume Content (FVC), Two-dimensional (2.3) \\
\hline $\mathrm{w}$ & Kink Band Width (2.1) \\
\hline $\mathrm{w}_{\mathrm{y}}$ & Average Width of Yarn (3.4.2.1) \\
\hline
\end{tabular}




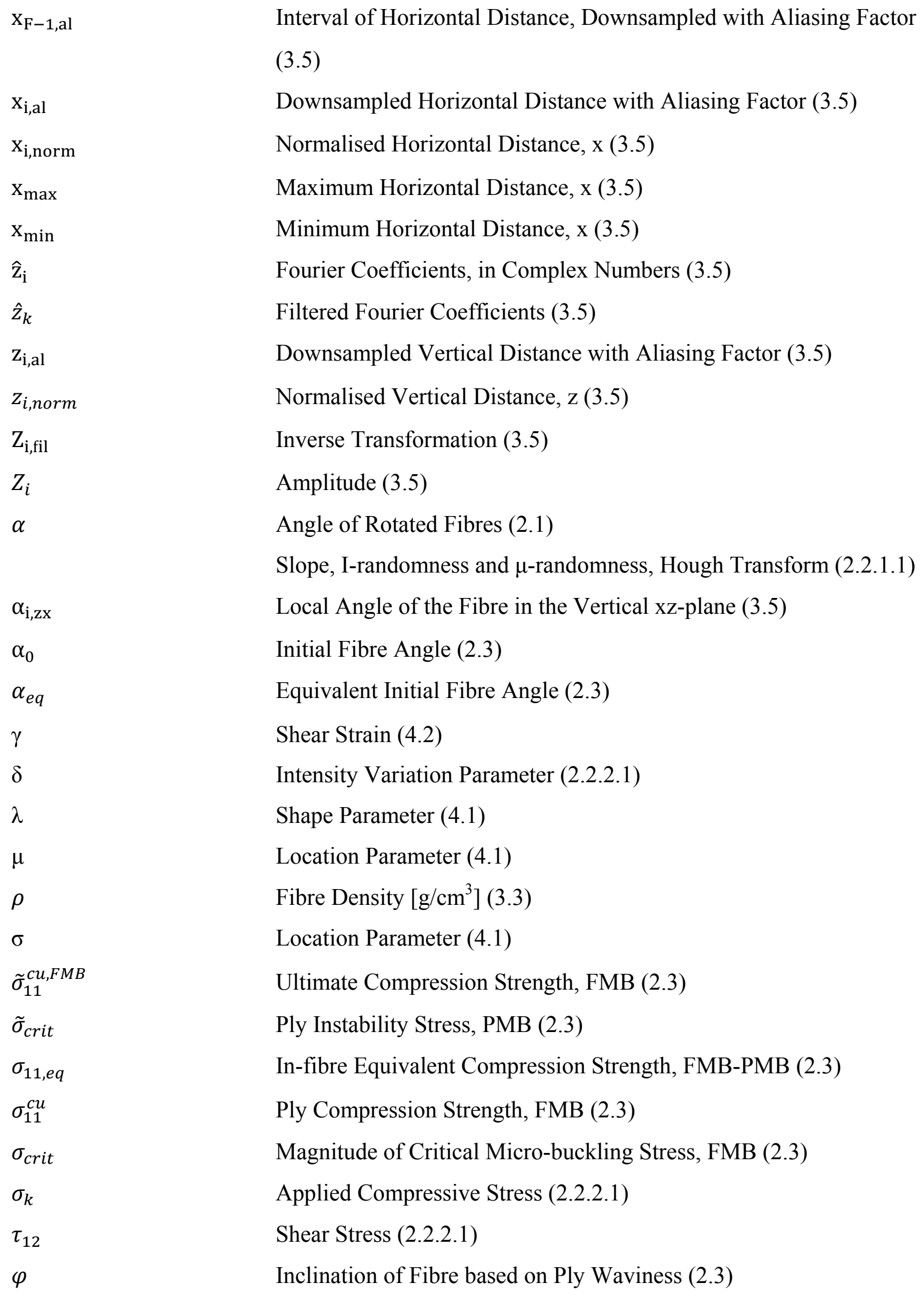


$\epsilon$

$\varphi_{i}$

$\omega$

$f(\omega)$

$\psi(\alpha)$
FVC (3.3)

Phase (3.5)

Fibre Angle Misalignment (2.2.2.1)

Distribution of Fibre Angle Misalignment (2.2.2.1)

fibre orientation distribution (ODF) (2.2.1.1) 


\section{CHAPTER 1: INTRODUCTION}

\section{OUTLINE OF CHAPTER 1}

- Different classification of composite material systems will be explained, ex: particulate, discontinuous, continuous.

- Continuous fibres: Carbon Fibre Reinforced Plastic (CFRP) composites are the focus of this study.

- There are two types of CFRP composites: Multiaxial Warp Knitted (MWK) fabrics and Prepregs. MWK fabrics, a type of Non-Crimp (NC) fabric, are the focus of this study.

- Resin Transfer Moulding (RTM) and Vacuum Infusion are two processes that can be used to create carbon composite laminates.

- Prepreg unidirectional (UD) tapes can be also used to create CFRP composites, just as MWK fabrics. Prepregs have been widely investigated and perfected. MWK fabrics are newer and not as well understood.

A composite is a material made of two or more phases. The combination of these two phases has mechanical properties and performance better than the individual phases alone. The stiffer phase is known as the reinforcement and the weaker phase is known as the matrix. How uniformly or homogeneously the reinforcement is distributed in the matrix determines the variation in material properties, thus failure (Daniel \& Ishai, 2006).

Although composites can be found in the human body, plants, and animals, its application is very new. It was not until the early 1960's that scientists and engineers have tried to fabricate composite materials (Herakovich, 1998). Materials can be placed into three broad categories: metals, ceramics and polymers. Accordingly, the reinforcement and the matrix can be made of metal, ceramic, or carbon. Polymers, such as epoxy resin, can only be used as matrices. There are two types of polymers: thermoset and thermoplastic. Thermoset polymers, the most common, decompose upon reheating whereas thermoplastic polymers soften upon reheating. Fibrereinforced composites, the focus here, can have polymer, metal, ceramic, or carbon based matrices (Daniel \& Ishai, 2006). The different combinations of these materials are chosen with care to maximise structural efficiency. 
There are many applications of composite materials: aerospace and aircraft, automotive, sports equipment, infrastructure structures such as bridges and buildings, and biomedical applications. Of particular interest here is how composite materials are used in the aerospace and aircraft industry. The lightweight, high stiffness, dimensional stability, corrosion resistance, design adaptability, durability and reduced maintenance characteristics of composite materials make them indispensable in the aerospace and aircraft industry (Daniel \& Ishai, 2006), as shown in Figure 1.

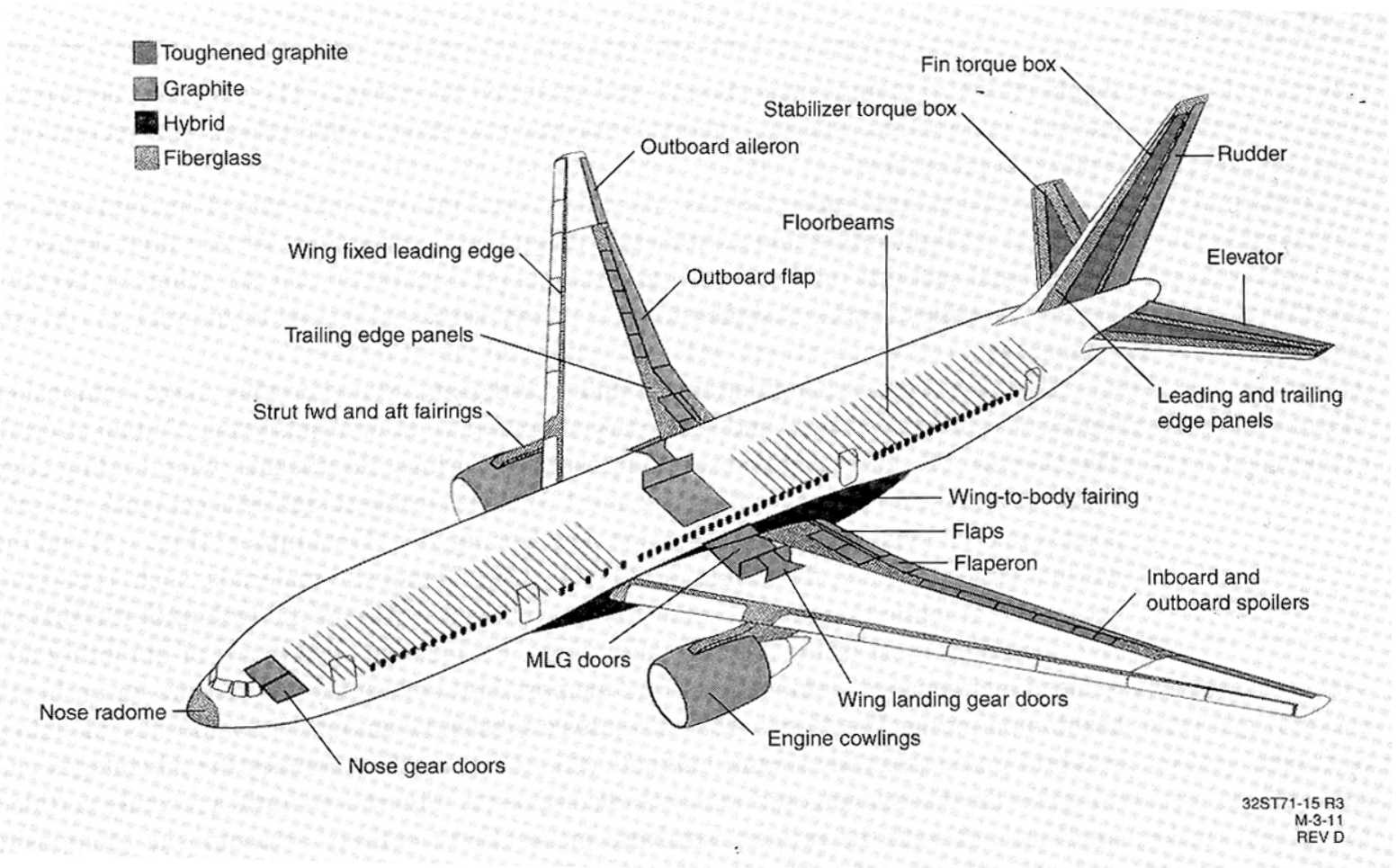

Figure 1: Usage of composite materials in various components of the Boeing 777 aircraft (Daniel \& Ishai, 2006)

\subsection{MATERIAL AND FABRICATION}

Composites can be made into flat plates, "laminate plates," with each laminate composted of many lamina or plies, or formed into complex, curved geometries. There are different ways to make these structures, depending on how the reinforcing fibre is used.

As Figure 2 illustrates, the reinforcement can be in the form of particles, discontinuous fibres, or continuous fibres. An example of reinforcement with particles is concrete reinforced with mica flakes; an example of reinforcement with discontinuous fibres are nano-composites 
reinforced with carbon nanotubes; an example of reinforcement with continuous fibres are Carbon Fibre Reinforced Plastic (CFRP) plates reinforced with carbon fibres and filled with epoxy resin. For the latter, the fibres can be arranged parallel to each other, as in the case for unidirectional (UD) laminates, arranged $90^{\circ}$ from each other to form a weave or crossply that has one layer stacked above the other, or arranged in multi-directions. In the case of multidirectional continuous fibre composites, the layers will need to be stacked with different fibre orientations for each layer (Daniel \& Ishai, 2006). Alternatively, the fibres could be knitted or braided.

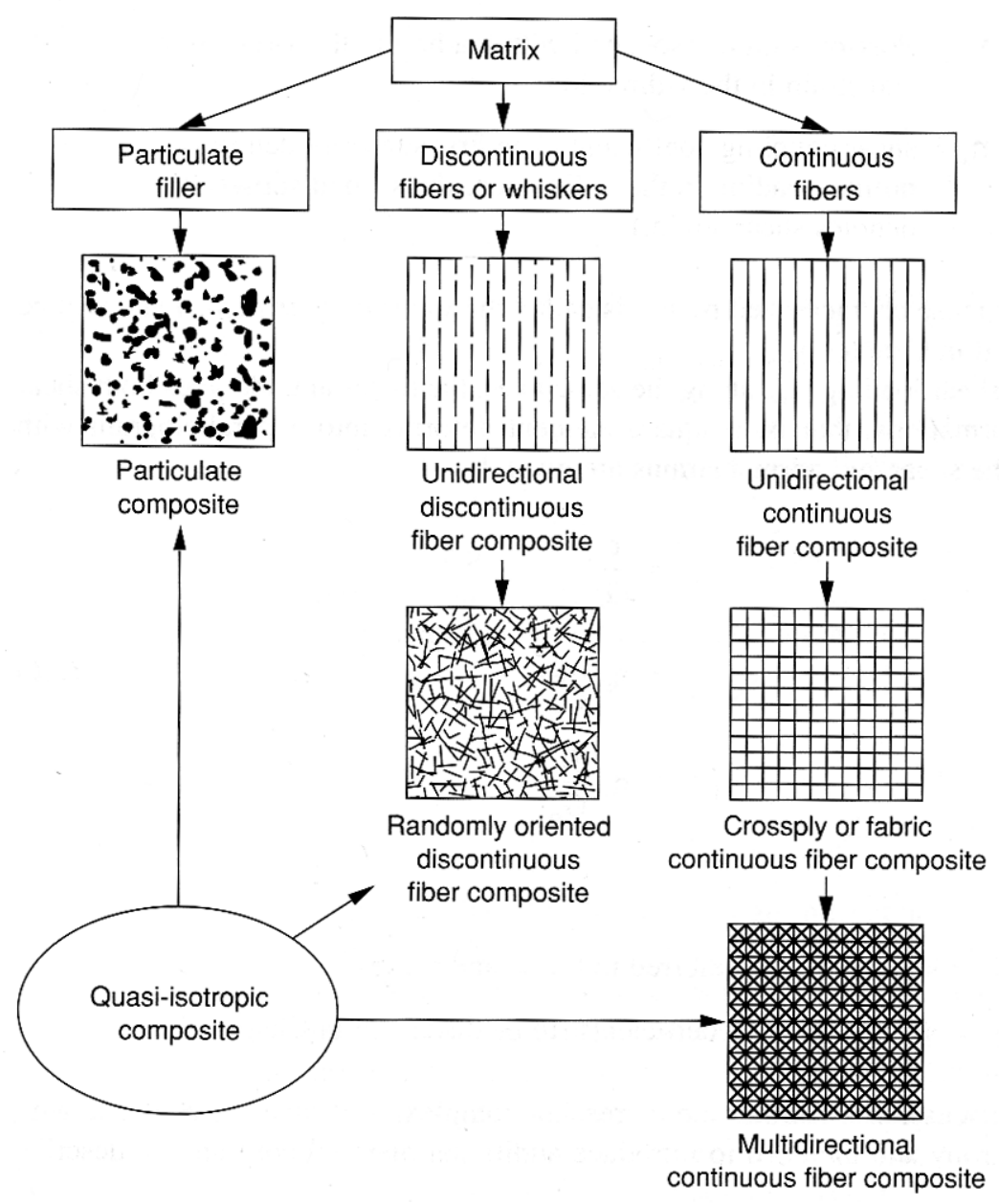

Figure 2: Classification of composite material systems (Daniel \& Ishai, 2006) 
As shown in Figure 3 to Figure 5, the reinforcing fibre can be classified as linear for the case of particulate composite, unidirectional discontinuous fibre composite and unidirectional continuous fibre composite, two dimensional for crossply or fabric continuous fibre composite, and three dimensional for multidirectional continuous fibre composite (Mallick, 1997).

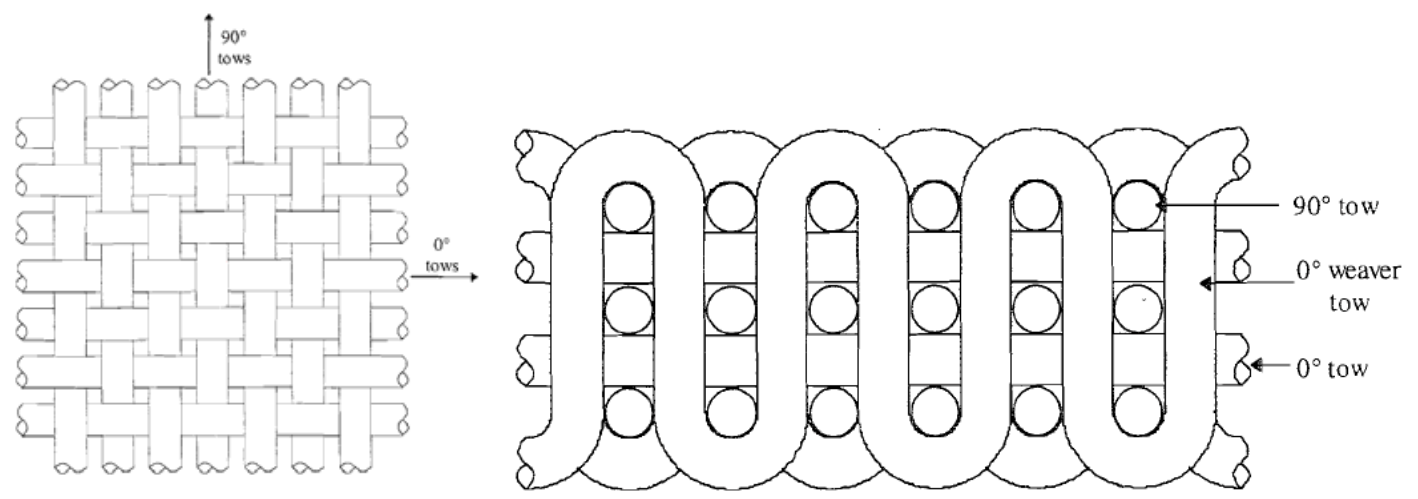

Figure 3: 2D orthogonal weave fabric (plain weave), 3D orthogonal woven fabric (Miller, 1996)

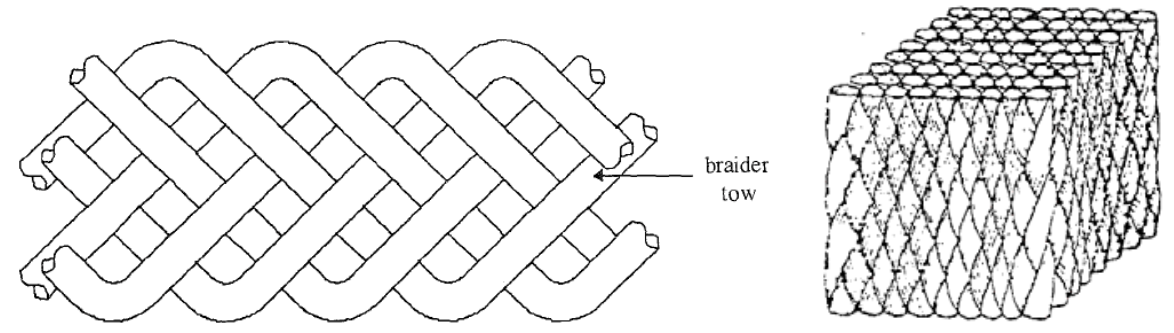

Figure 4: 2D braid, 3D braid (Miller, 1996)

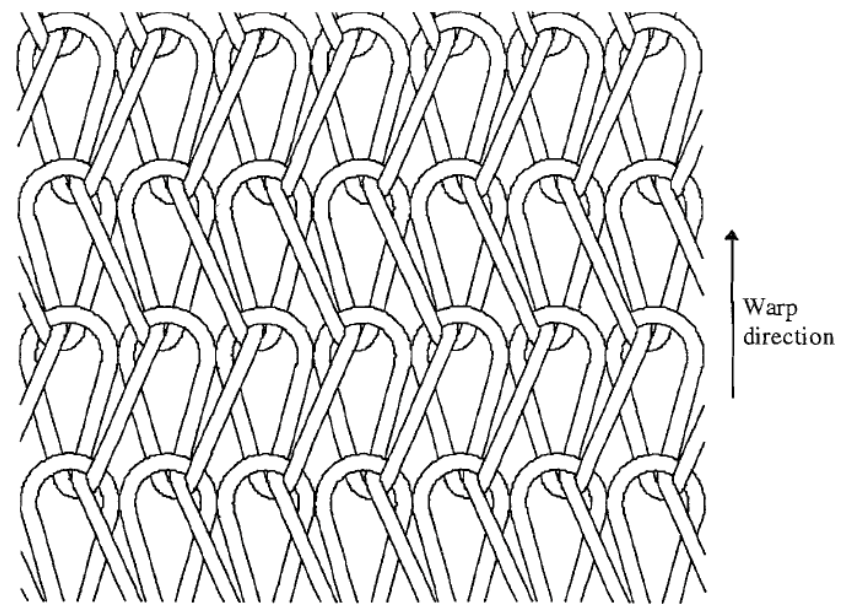

Figure 5: Knitted fabric, Warp (Tricot) Knit (Miller, 1996) 
Carbon Fibre Reinforced Plastic or Polymer (CFRP) laminates, are of particular interest in this study. There are two types: Non Crimp Fabrics (NCF) and prepreg (preimpregnated tape).

Non Crimp Fabrics (NCF) used for Carbon Fibre Reinforced Plastic or Polymer (CFRP) laminates, are of particular interest in this study. Non Crimp Fabrics (NCF) is another name for Multiaxial Warp Knitted Fabrics (MWK), originally developed for the marine and construction industries (Bibo, Hogg, \& Kemp, 1997). As shown in Figure 6, Multiaxial Warp Knitted Fabrics can be used to create a type of multidirectional continuous fibre composite. They are composed of one to four layers of parallel fibres arranged at different orientations from each other, then stitched together with a thread that is made of polyester, forming a fabric. Here, the stitching pattern is known as "warp knitting" because many separate pieces of the thread, also known as yarn, runs in a zig-zag or straight pattern along the fabric, interlinked in the form of a chain on the back side of the fabric. If the fibre is arranged along the zig-zag pattern, this is called the warp $\left(0^{\circ}\right)$ direction, versus the filling direction $\left(90^{\circ}\right)$ (Mallick, 1997).

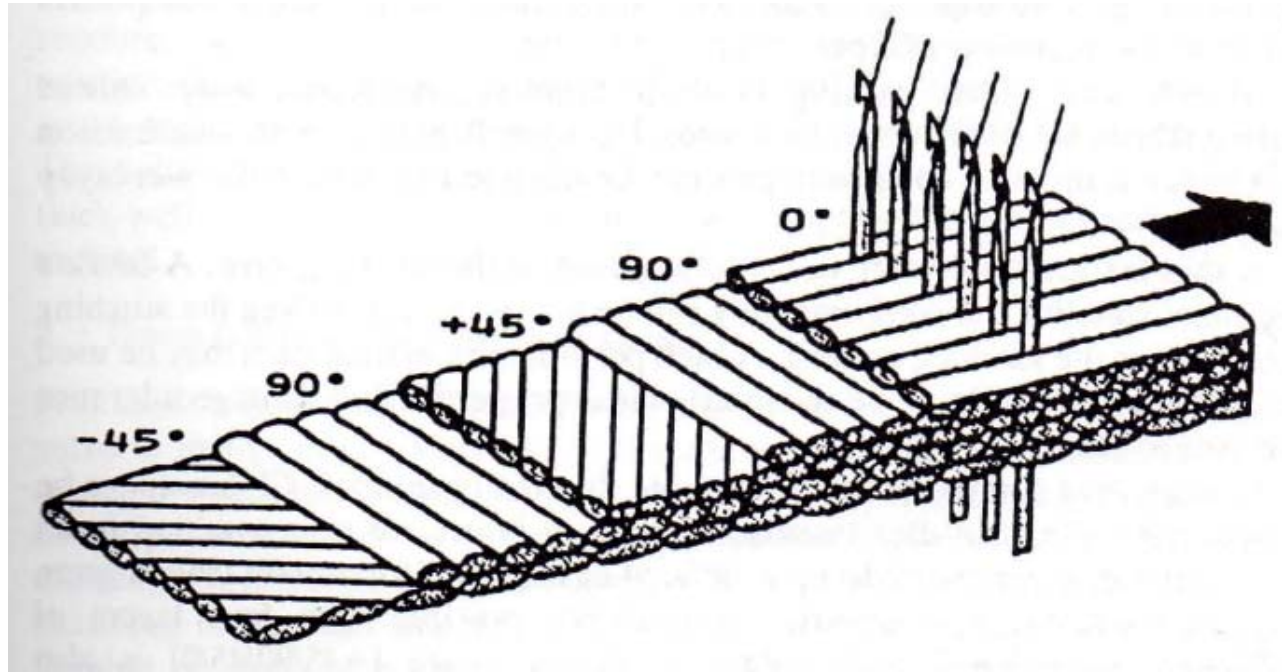

Figure 6: Schematic of multiaxial warp knitting processing (Hörsting, Wulhorst, Franzke, \& Offermann, 1993)

Multiaxial Warp Knitted (MWK) fabrics allow for fibre movement and helps maintain uniform fibre spacing within the fabric, making them the ideal candidate for constructing complicated shapes without much pre and post processing: multiple layers are handled in a single step and post-consolidation machining is eliminated. They allow for close fibre packing, thus creating a high fibre volume fraction (Mallick, 1997). Most importantly, they keep the fibres straight, thus taking full advantage of the stiffness of the fibres since a woven fibre would have compromised stiffness, thus the name "Non Crimp Fabric." 
Resin Transfer Moulding (RTM) and Vacuum Infusion can be used for creating the part. RTM involves enclosing the fabric and two sides of the mould in a heated press, infusing the setup with the matrix resin, and applying pressure. Vacuum Infusion involves a single sided mould with vacuum bag on the other side, placing the set-up in a hot air oven, placing another vacuum bag on set-up, keeping the resin pot at ambient pressure, and not applying elevated pressure. These are much less expensive manufacturing process compared to using an autoclave, which can be quite huge and expensive to acquire and to maintain.

Resin Transfer Moulding (RTM) can also be used for woven fabrics. In addition to RTM, Pultrusion, which involves pulling fibres and fabrics through a bath of liquid resin, through formers that align the material to their desired configuration, and then to a heated die can be use. Discontinuous fibres are made into Sheet Moulding Compound (SMC), which are sheets of matrix resin with discontinuous fibres inside, a type of semi-finished part. In the case where the matrix does not need to be injected into the fibre, the autoclave is used. This is the situation with filament winding in which matrix coated fibres are wound around a mandrel with the desired final shape, with braiding in which the matrix coated fibres are knitted in curved geometries, and with prepreg tapes. Prepregs are expensive materials, very thin, about $0.125 \mathrm{~mm}$ thick, which mean creating thick parts will be extremely time consuming, and need to be carefully stored in freezer (Herakovich, 1998), making NCF's an ever more attractive alternative.

Prepreg tapes, as previously discussed, is another type of semi-finished part to produce Carbon Fibre Reinforce Plastics (CFRP). Pre-impregnation of the resin in the fibre can come in the form of UD prepreg tape, prepregged NCF, and prepregged woven fabric. It has been found that laminates made with prepreg NCF and the prepreg woven fabric had a much lower unnotched compression strength (-30\%) than those made with UD prepreg's. This is a result of "crimping" due to the stitching of the thread through the fabric, the thread that keeps the layers together. The tensile strength, on the other hand, showed little difference, as long as the sewing thread is not too thick. Further investigations of performance differences between prepreg's and NCF's, NCF's with aviation-qualified high strength (HS) (tensile modulus between 317 to 394 $\mathrm{GPa}$ ) or intermediate strength (IM) (tensile modulus between 255 to $317 \mathrm{Gpa}$ ) carbon fibres in combination with highest performance epoxy resin systems, revealed that the notched tension strength of NCF's outperformed prepregs's $(+20 \%)$; notched compression strength was worse for NCF's $(-15 \%)$, as well as bolt-loaded bearing strength $(-30 \%)$, and compression strength after 
impact (-30\%). Deficiencies in the bearing strength were revealed to be due to compression strength and that compression strength after impact is due to the type of matrix used. In prepregs, some thermoplastic additives are used, which increases their fracture toughness, thus compression strength after impact. From all this, it can be seen how much compression strength is severely affected in NCF's, when compared with prepreg's, an older technology (Pansart, 2008).

As a result, areas that might be investigated in composites include: the creation of curved and cylindrical geometries, the permeability of the carbon fibre fabric/textiles, and the curing properties of resins and adhesives. 


\section{CHAPTER 2: LITERATURE REVIEW ON COMPOSITE DEFECT}

\section{OUTLINE OF CHAPTER 2}

- Defect in terms of fibre kinking and micro-buckling will be described.

- Non-Destructive Techniques (NDT) of investigating defects will be discussed. These techniques involve analysing scanned images for fibre orientations, "fisheyes," and other defects.

- There are many ways to find the fibre orientation: statistical method, direct tracking, Hough transform

- Other defects include: pilling and wrinkling, which apply more to fabrics used to make clothing.

- Non-Destructive Inspection (NDI) will be discussed, with respect to investigating and repairing an aircraft that have entered service.

- Destructive Techniques (DT) for investigating defects will be discussed. These include using optical microscopy to find the fibre orientation, and compression and tensile testing using a coupon (sample) in the laboratory.

- Several methods of predicting compression strength of CFRP laminate will be discussed and the ones used for this study have been thoroughly described: the FMB-PMB model and the Unit Cell Model.

- The way reliability has been used with respect with quality control of CFRP laminates will be discussed.

- The objectives of the thesis are listed at the end of this chapter.

Due to the strict requirements of composites for aerospace applications, having a reliable way for understanding and detecting defect needs becomes important. The next Section, 2.2, discusses the existing theory behind composite defect, in terms of kinking and micro-buckling. The next Sections, 2.2.1 to 2.6, discuss non-destructive and destructive techniques for investigating defect, existing models to predict composite failure, some of the applications of reliability in composites, and the objectives of this thesis. 


\subsection{UNDERSTANDING DEFECT}

In a study (Schultheisz \& Waas, 1996) to understand defect, it was found that there was complexity in predicting compression strength due to structural instability such as material geometry and stress concentrations. Stress concentrations can cause failures within the grips of the test specimen instead of the test section. It has been found that the matrix plays important role in resisting compressive failure because dry fibres do not support compressive load (Mallick, 1997).

In composite materials, compression strength is lower than tensile strength, which limits its application. Carbon fibres are anisotropic, therefore, compression strength maybe only $10-50 \%$ of tensile strength depending on precursor and heat treatment of fibre. Also, there is geometrical and material nonlinearity. As the result, there is great need to accurately predict compression strength.

Usually, two dimensional laminates are built, therefore, Classical Laminate Theory (CLT), only for thin laminates (36-48 plies), can be used. However, CLT is only for anisotropic materials, not for isotropic material. Therefore, it cannot predict material breakdown.

In the case with unidirectional (UD) composites under uniaxial compression, there exist some micromechanical failure theories. These theories look into the individual fibres. Predominant failure modes include: micro-buckling and kinking. It is possible for the failure modes to combine and certain failure modes to dominate for certain conditions. Also, the test method may induce stress concentrations, thus induce a particular failure. These failure modes most often caused by initial imperfections from manufacturing: fibre misalignments, waviness, residual stresses, and porosity.

Micro-buckling is the most studied failure mode. In high volume fraction composites, micro-buckling can be found in matrix stiffness in shear that is affected by time, history, strain rate, and the environment. The micro-buckling occurs when the fibre compression strength is greater or equal to the tensile strength of carbon or glass fibres.

Rosen (1965) showed fibres act individually as columns in matrix, with the each fibre forming layers (plates) that are perfectly straight and evenly spaced. The fibre and matrix are linearly elastic and failure is expected to occur in two ways: extension mode and shear mode, 
with the matrix predominantly in extension (out-of-phase deformation) or shear (in-phase deformation). As shown in Figure 7, micro-buckling is assumed to occur everywhere in the composite at about the same time.

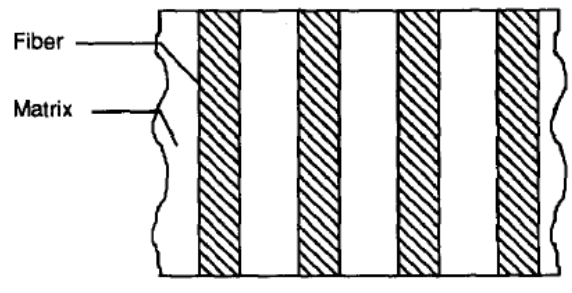

a.) Initial Configuration

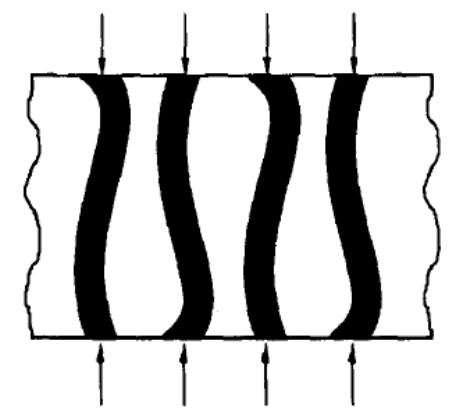

b.) Extension Mode

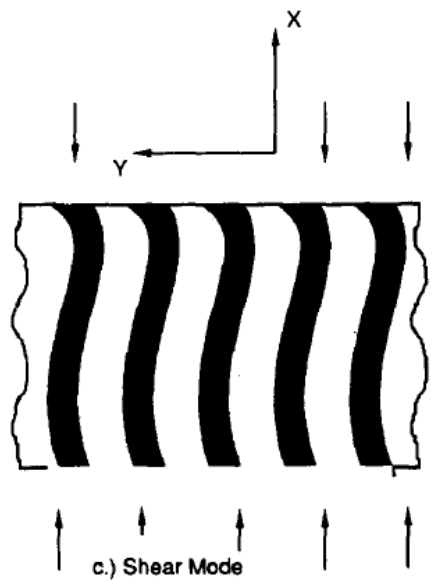

Figure 7: Rosen's Fibre Micro-buckling Model (Schultheisz \& Waas, 1996)

Kinking is defined to be fibre fracture and localized shear deformation of the matrix that is often considered to be the final, irreversible, consequence of micro-buckling, only for the case of very soft matrices at high temperature. Fibre failure or collapse can be easily identified. It is only for weak fibres such as Kevlar. Strong fibres such as glass, carbon, and boron would not experience fibre fracture. Meanwhile, strong fibres in weak matrices produce longitudinal cracking, which is influenced by fracture toughness of matrix or interface, mismatch in Poisson ratio between fibre and matrix, and contaminated resin, forming an "interphase" between fibre and matrix (Rosen, 1965).

In kinking, deformation occurs in the form of a band across specimen where fibres have rotated a large amount, causing matrix-shearing deformation. Figure 8 shows the kinking parameters, which are: kink band width, w, angle of rotated fibres, $\alpha$, and angle of kink band, $\beta$. The kink band width decreases with specimen width. The fibres are usually broken at the kink 
band boundary, but weak fibres such as Kevlar may not break. Complementary kinks may occur to relieve transverse offset cause by a single kink band (Schultheisz \& Waas, 1996).

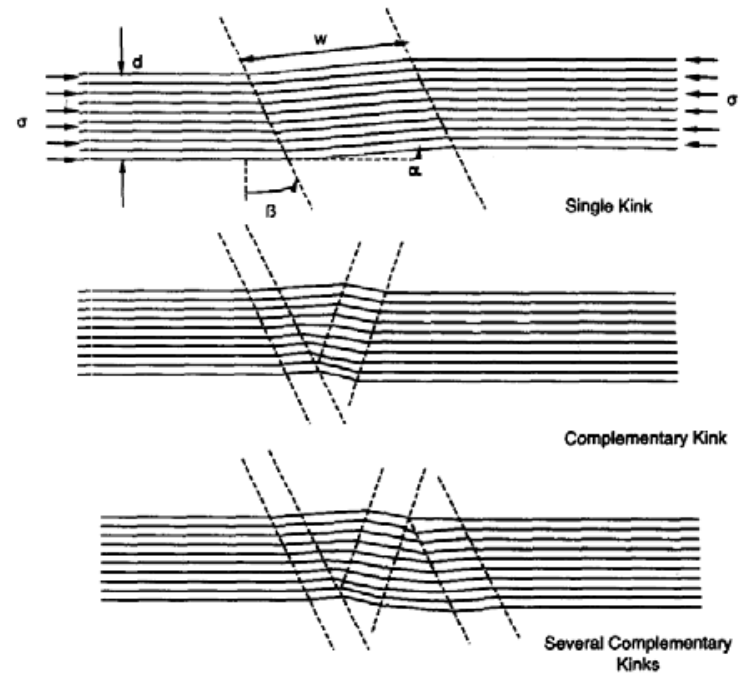

Figure 8: Geometry of kink bands (Schultheisz \& Waas, 1996)

Kinking occurs from some kind of defect and is most commonly found after testing. It is catastrophic and has a short duration. It could be caused by sudden event such as fibre fracture, localised fibre buckling, and matrix shear, which propagates to undamaged regions. There is plastic deformation of the matrix that is initiated through fibre collapse (Kevlar fibre), fibre fracture (carbon) or matrix yielding (glass) (Schultheisz \& Waas, 1996).

Argon (1972) investigated the relationship between local initial fibre misalignment and kink band formation. Chaplin (1977) found that the angle of kinked fibres $(\alpha)$ is related to angle of kink band ( $\beta$ ), $\alpha=2 \beta$. Also, it was found that with $\alpha$ between $48^{\circ}$ and $67^{\circ}$, the kink width is related to tensile breaking strength of fibres (Evans \& Adler, 1978). This is under combined compression and bending in the kink band. Here, the stress to propagate the kink band is approximately two orders of magnitude less than the stress required to initiate kinking through micro-buckling (Steif, 1990a,b). However, further investigations indicated that wavy fibres become kink bands only if there is local matrix cracking (Schapery, 1991). Barbero (2008) used the Weibull distribution to describe the dispersion of fibre stress under tension, the stress that the fibres are able to withstand, at a particular fibre length. Barbero (2008) also used the Gaussian distribution to describe the probability of finding a fibre with misalignment angle, $\alpha$, for the fibre buckling strength. 
Poor adhesion between fibre and matrix can cause longitudinal cracking (delamination) and fibre compression failure. However, poor adhesion between fibre with different surface treatments (different interfacial strength) and matrix can cause micro-buckling or kinking. The adhesion between fibre and matrix, interfacial strength, can be used to determine the failure mode. With the lowest interfacial strength, the failure mode is longitudinal cracking, meanwhile, with intermediate interfacial strength, micro-buckling or kinking, with highest interfacial strength, fibre compression failure (Madhukar \& Drzal, 1992). It has been found that longitudinal cracking is a precursor to kinking (Berg \& Salama, 1973). Therefore, kinking could be due to micro-buckling.

It has been proven that Rosen's model overestimates the failure stress of most composite materials and this could be due to the fact that the three dimensionality of structure is neglected. This overestimation occurs by a factor of 2 to 3 for carbon composites (Weaver \& Williams, 1975; Wronski \& Parry, 1982).

\subsection{DEFECT DETECTION}

There are different ways to investigate how defect occurs in composites: Non-destructive Testing (NDT) and Destructive Testing (DT). Also, there are theoretical models that predict failure under tension and compression, in which failure due to compression will be the focus of Sections 2.2.1 to 2.2.2.2.

\subsubsection{Non-Destructive Testing}

Non-destructive Testing is unlike Destructive Testing in which a laminate is not manufactured and then destroyed to determine its ability to withstand tensile and compressive stress. The first part of this Section, 2.2.1.1, will discuss the analysis of images of textiles. This is used to find properties such as fibre orientation, as an indication of textile quality. The last part, 2.2.1.3, will briefly discuss Non-Destructive Inspection (NDI) of components that have entered into service.

\subsubsection{Fibre Orientation}

Based on Miene, Herrmann, and Göttinger (2008), a study was performed to analyse scanned images of textiles in order to find the average and standard deviation of fibre orientation for each textile. A larger standard deviation would indicate a MWK fabric that has a more 
"wavy" arrangement of fibres, which could potentially lower the compression strength of the manufactured laminate. The described method, which is one of the methods of how fibre orientation measurements have been measured in this thesis, is based on using image processing algorithms from Intel ${ }^{\circledR}$ Computer Vision Library (OpenCV). This led to the development of software known as "Fibre Image Analysis," which will be discussed in detail in Section 3.4 in Chapter 3. Other computer algorithm-based methods have been used to measure fibre orientation, such as: Direct Tracking and Hough Transformation, which will be discussed in greater detail.

Miene et al. (2008) used a method that uses gradient filters to measure local fibre orientations, the direction histograms, the distribution of edge orientation, where the edge would represent the fibre in this situation. It is a statistical approach based on Tamura, Mori, \& Yamawaki (1978). The purpose is to find the texture direction histogram, which is the probability of local edge direction angles over the entire image. Here, $\theta$ is the edge direction, with a range of $[0, \pi]$, measured counter clockwise, where the horizontal is zero, and $|\Delta G|$ is the edge magnitude. Both are calculated from gradient operators $\Delta x$ and $\Delta y$. $\mathrm{A}$ is the source image.

$$
\begin{aligned}
& |\Delta G|=\sqrt{\Delta_{x}^{2}+\Delta_{y}^{2}} \\
& \theta=\tan ^{-1}\left(\frac{\Delta_{y}}{\Delta_{x}}\right)+\frac{\pi}{2} \\
& \Delta_{x}=\left[\begin{array}{ccc}
-1 & 0 & +1 \\
-1 & 0 & +1 \\
-1 & 0 & +1
\end{array}\right] \cdot A \\
& \Delta_{y}=\left[\begin{array}{ccc}
-1 & -1 & -1 \\
0 & 0 & 0 \\
+1 & +1 & +1
\end{array}\right] \cdot A
\end{aligned}
$$

The range of directions is placed into $k$ intervals and the edge direction is counted in the direction histogram only when $|\Delta \mathrm{G}|$ exceeds a threshold, $t$. In order to find the edge direction histogram, a Prewitt operator/filter is used, with similar formulas as in 2.3 and 2.4. Tamura et al. 
(1978) proposed to use a $3 \times 3$ Prewitt operator with $k=16$ (16 texture orientations) and $t=12$. However, for the case of Miene et al. (2008), more accuracy was needed. Therefore, either a $5 \mathrm{x}$ 5 or $7 \times 7$ Prewitt operator was used with $k=180$.

In addition to finding the fibre orientation of the textile, the "sewing threads" used to sew MWK textiles together need to be identified and removed from the direction histogram because they are usually oriented in an opposing direction from the fibre. The "sewing threads" are identified after a local adaptive binarization of the image, when the pixels of the image has been turned into black or white.

The resulting texture direction histogram is usually "bell" shaped, with a peak that identifies the most measured angle. An image can be split into many regions, called domains. The image region is used to find the texture direction histogram, the probability of local edge direction angles over the entire image. The size of the domain can have an impact on whether the resulting histogram has a smooth peak and is statistically significant. If the histogram is not smooth, noise has to be removed. In addition to domain size, the quality of the image can influence the direction histogram, especially sharpness and illumination, and the amount of fibre in the domain.

Due to the above mentioned problems that could cause falsely calculated fibre orientations, two criterions are used: 1) standard deviation of the angles within each domain, and 2) percentage of edge (fibre) pixels in each domain. A large standard deviation means that the peak is very flat and there may be arbitrary angles. A low percentage of fibre pixels in a domain means that the image region captured by the domain is blurred or is at the edge of the textile where part of the background (not textile) is included in the analysis. A dark vector would be created in the domain that represents the calculated fibre orientation if either one of these criteria is violated. Otherwise, the vector would be light.

In addition to the two criteria mentioned above, a tolerance-based warning system is used to identify how good the measured fibre orientation is. Two angle orientation tolerances are specified, where Tolerance \#1 is less than Tolerance \#2. If the resulting domain has results that fall below both tolerances, then the vector in the domain is green. If only the first tolerance is exceeded, a yellow warning vector results. If both tolerances are exceeded, a red "error" vector results. 
In addition to the two criteria and the tolerance-based warning system, the fibre orientation can be locally compared within each domain to an expected reference matrix, and/or globally compared within the entire image to an expected fibre orientation.

The process that Miene et al. (2008) used is similar to the process used by Tamura et al. (1978). In both situations, the same directionality equation, equation 2.2 , is used. In addition to directionality, Tamura et al. (1978) also looked at five other basic textural features using computer software: coarseness, contrast, line-likeliness, regularity, and roughness. These features were compared to human visual perceptions and have resulted in a good correlation to digital analysis, despite of some discrepancies that some can be resolved through more sophisticated techniques.

Tamura et al. (1978) define textures in a macroscopic region as a function, in which its elements are arranged according to a placement rule:

$$
f=R(e)
$$

Where,

$R$ is placement rule or relation

$e$ is element

$f$ is texture

Given this definition of texture, microtexture is when $f$ can replace $e$ recursively. Texture can be defined in two levels: statistical and structural. For statistical texture, statistics are taken local properties, from naturally occurring textures, and generally describe textures with much variation. As a result, $R$ and $e$ also have much variation, and cannot be separately approximated. Therefore, statistics are extracted from local measurements. Quite contrarily, structural texture describes textures without much variation. There is more information about $R$ and $e$ that help define them. Only a part of the infinite number of texture patterns can be distinguished by this method. It is possible that $R$ and $e$ have statistics that interfere and cancel each other. Despite of this, for practical applications, a simplified and computationally easier statistics can be used, for example, Haralick’s “grey-level co-occurrence matrices” (Tamura et al., 1978).

According to Tamura et al. (1978), the computational measures were compared to results from human subjects, 28 men and 20 women. This part of the study was a psychological experiment, in which 16 patterns were digitized to 256 × 256 pixels, 64 bit grey level, shown on a 
CRT monitor, and compared using pair comparison, which is commonly used in the psychometrics field, to find the pattern that coarser, higher in contrast, etc, based on the six textural features mentioned earlier. These results were then further processed using "the law of comparative judgment" (Thurstone, 1927) to create a one dimensional scale for each feature. Finally, these results were compared to the authors' results before being compared to the computational measures. This was performed using Spearman's coefficient of rank correlation:

$$
r_{s}=1-\frac{6}{n^{3}-n} \cdot \sum_{i=1}^{n} d_{i}
$$

Where,

$d_{i}$ is the difference between ranks in the $i^{\text {th }}$ object in two measurements

$n$ represents the 16 different pair wise combinations between the textural features

From this calculation, it was found that there were some discrepancies between the judgment of Tamura et al. (1978) and the human subjects, most likely due to inaccurate definitions of the six features, especially in the definition of "regularity."

For each of the six texture features, different specific formulations were defined, in the form of equation 2.5. Of particular interest here is the definition by Tamura et al. (1978) for directionality, which resembles equation 2.1:

$$
|\Delta G|=\frac{\left(\left|\Delta_{H}\right|+\left|\Delta_{V}\right|\right)}{2}
$$

The definition by Tamura et al. (1978) for $\theta$ is the same as equation 2.2.

Here, $\Delta_{H}$ is the horizontal difference and $\Delta_{V}$ is the vertical difference and $0 \leq \theta<\pi$, measured counter clockwise. The formulation for the "Directionality Histogram" in the form of equation 2.5 is:

$$
H_{D}(k)=\frac{N_{\theta}(k)}{\sum_{i=0}^{n-1} N_{\theta}(i)}
$$

Where,

$k=0,1, \ldots, n-1$ and $N_{\theta}(k)$ is the number of points which satisfies $\frac{(2 k-1) \pi}{2 n} \leq \theta<\frac{2 k+1}{2 n}$ 
Like in the work by Miene et al. (2008), there is a threshold, $t$, for the gradient: $|\Delta G| \geq t$

Tamura et al. (1978) used $n=16$ and $t=12$. As a result, the histogram, $H_{D}$, is not sensitive to the threshold value. As in the case with Miene et al. (2008), the peak of the histogram determines the directionality. It is possible that there is more than one peak. If there are two peaks, this means that the analysed image is bidirectional.

During the test of similarity, images were grouped together. The results of human subjects and the computer were compared. This resulted in large differences, which indicates an area that is still in need of further development.

Another study was performed to find the "directionality" in an image. This study resulted in a six part journal series. The series started with the creation of simulated images with different orientations. Eventually, "Real Web" fabrics were analysed. Finally, different methods were proposed to find the directionality: "Direct Tracking," "Fast Fourier Transform," and the "Hough Transform." A selection of these journal papers will be summarised in the next paragraphs.

In Part I of the series by Pourdeyhimi, Ramanathan, \& Dent (1996a), computer simulated images with continuous and discontinuous fibres were created. They were used as a point of comparison with different algorithms that will be mentioned in the series to find the fibre orientation. Two procedures were introduced to create these simulated images: I-randomness (internal randomness) and $\mu$-randomness (mean free path).

I-randomness has been found to work well in the creation of images with discontinuous (staple) fibres due to unbiased, which are spatially homogenous, arrays. In this procedure, point $P(x, y)$ is randomly chosen in a plane larger than the image by length " $\ell$." Then, the slope according to angle, $\alpha$, is sampled from a distribution. The point $P(x, y)$ is chosen as the centre of mass of the resulting line, with total length " $P$ " and slope $\alpha$, as shown in Figure 9.
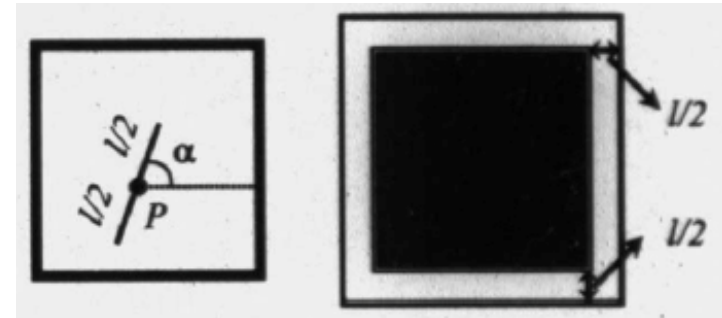

Discontinuous

Figure 9: I-randomness Procedure for Discontinuous Fibres (Pourdeyhimi et al., 1996a) 
The $\mu$-randomness procedure has been found to work well in the creation of images with continuous fibres for unbiased arrays. In this procedure, a reference point is chosen, $O$, preferably at the centre of the image. The slope, $\alpha$, is sampled from a distribution, which represents the angle drawn from the horizontal, $0^{\circ}$, position to $d$. The length of distance, $d$, is sampled from a distribution that is limited to the diagonal of the image. A continuous line is drawn perpendicular to $d$ as shown in Figure 10 .
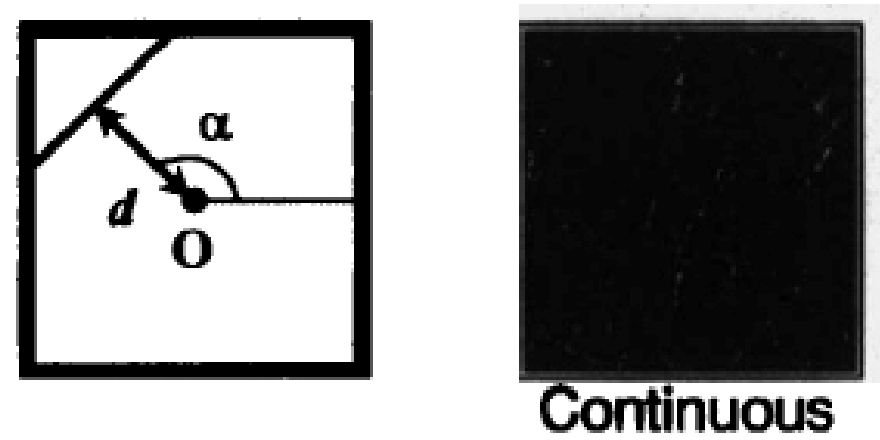

Figure 10: $\mu$-randomness Technique for Continuous Fibres (Pourdeyhimi et al., 1996a)

In addition to these two procedures to create simulated images, a sine wave is used in conjunction with these procedures to model crimp or wave of the fibre. Other key structural parameters have been taken into account are: web density, fibre orientation distribution (ODF), fibre diameter, and fibre length. For the fibre orientation distribution function, there is a segment length limit for a particular slope. This is the chord orientation calculated from the first and last point. Pixel deviations from the ideal line are errors. Errors are minimized with a length greater than 20 to 24 pixels.

The first method in the series that is used to find the fibre orientation angle is "Direct Tracking" (Pourdeyhimi, Ramanathan, \& Dent, 1996b). The method is a simple, rule-based, system. The speed of execution is slow, but works well with continuous, discontinuous, and crimped fibres. As depicted in Figure 11, the images must be binary, and each pixel is surrounded by eight pixels, allowing the direction of the line to proceed in eight possible directions. 


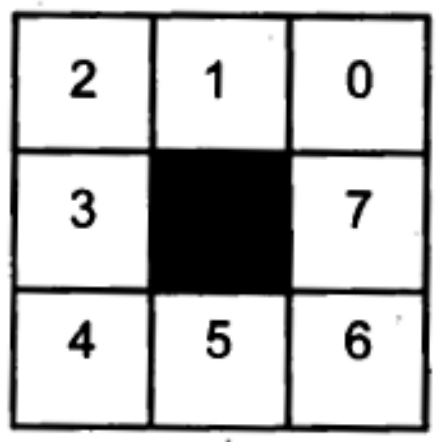

Figure 11: Square Raster Direction Coding (Pourdeyhimi et al. , 1996b)

The "property of the chord" applies to this procedure. This property basically states that in order for a digital line to be considered a digital line, there must be no more than a one pixel deviation from the digital line.

This procedure works by searching for direction between $-45^{\circ}$ and $45^{\circ}$. Given that there is more than one possibility for a search direction, a direction is chosen at random. The pixel with the least value of summed square deviations of all possibilities is taken as the next pixel in the track. The search ends when the image boundary has been reached or when the chord property is not satisfied. Also, a predetermined number of searches can be set. Fibres are tagged to prevent double counting. The chord length can be specified, and it has been found that a segment length of 24 pixels is suitable for straight and crimped fibres. These results were compared to the simulated images with specified orientation angles and it was found that this method is accurate and reliable.

Another method that can be used to extract the fibre orientation distribution (ODF), $\psi(\alpha)$, is through the use of the "Hough Transform" (Pourdeyhimi \& Kim, 2002). This method has been employed for nonwoven fabrics that can be used to detect straight and curved lines. Its use has been explored in the 90's, but with limited success due to limited computing speeds. It is now more feasible due to higher computing speeds.

The concept behind the Hough Transform evolves around the definition of a straight line:

$$
Y=m X+b
$$

Where, $X$ and $Y$ are defined as pixel coordinates, where $m$ is the slope and $b$ is the $y$ intercept. With the $y$-axis rearranged, equation 2.9 can be re-written as: 


$$
b=-X m+Y
$$

Where, $b$ and $m$ are the pixel coordinates, $X$ is the slope, and $Y$ is the $y$-intercept in the $(m, b)$ Hough space.

Due to this configuration, a point, $(x, y)$ generates a line in $(m, b)$ space. In addition to this, collinear points in $(x, y)$ space result in lines that intersect at a point in $(m, b)$ space and more lines intersecting in $(m, b)$ space means that there are more collinear points in $(x, y)$ space. However, since a vertical line has a slope of infinity, the normal form the line is used:

$$
x \cos \omega+y \sin \omega=r
$$

Here, $r$ is the perpendicular distance from $(0,0)$ to the line and $\omega$ is the angle perpendicular to the line formed between the $x$-axis as shown in Figure 12.

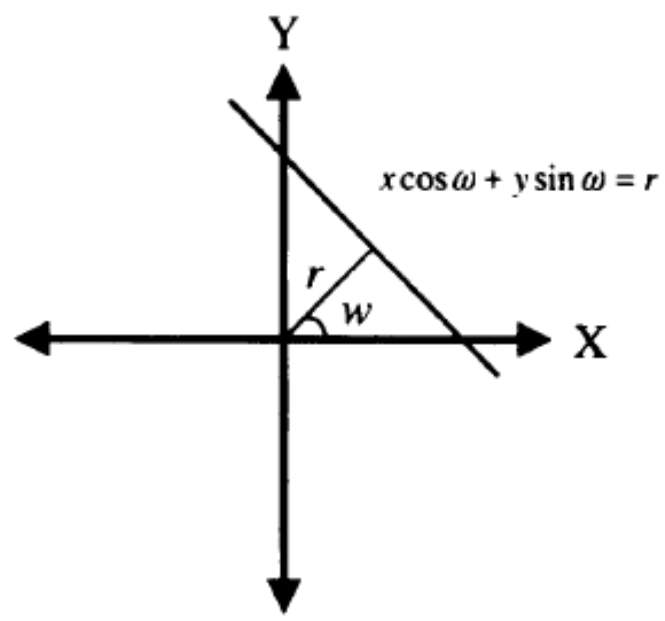

Figure 12: Line Representation and Corresponding Hough Transform (Pourdeyhimi et al., 2002)

To convert from slope-intercept form to normal form, the following equations are used with equation 2.11:

$$
\omega=\tan ^{-1}\left(-\frac{1}{m}\right)
$$




$$
r=b \sin \omega
$$

Where, $90^{\circ}<\omega<90^{\circ}$

It is possible for $r$ to have a maximum, as given in the following equation:

$$
r_{\text {max }}=\sqrt{(\text { image width })^{2}+(\text { image height })^{2}}
$$

As from before, each point in $(x, y)$ space is a curve in $(r, \omega)$ space and collinear points in $(x, y)$ are intersecting curves in $(r, \omega)$. Points of intersection in the Hough space, called peaks, indicate straight line segments in the $(x, y)$ space. It is important to note that collinear points separated by gaps correspond to the same peak. Therefore, the Hough transform method cannot measure length. The resulting Hough image would be covered in curves. The grey level intensity is used to find the relative frequency of the orientation angle. Given the correct filtering/ binning criteria of the orientation angle data to find the ODF, the result of the Hough Transform resembles that of the Fast Fourier Transform.

In the previous methods to find the fibre orientation distribution function (ODF), such as the "Direct Tracking" method and the Hough Transform, they were used on simulated images and their results were compared to the simulated images' parameters. Obviously, images of real textiles would present certain difficulties that simulated images would not. This is the case with real nonwoven fabrics, such as carded crimp fibre web, as discussed by Pourdeyhimi, Dent, Jerbi, Tanaka, and Deshpande (1999). Because there is more than one layer for these fabrics, the digitised microscope images at high magnifications are fuzzy due to the poor field of depth. Further, the reflection from the surface fibres can result in low contrast images. Therefore, the images will be non-uniform. The problem is resolved by an "on-axis" lighting system that passes the light through a diffuser and a beam splitter. The sample is placed on a mirror and the split beam of light passes through the fabric and is reflected to the camera as depicted in Figure 13. 


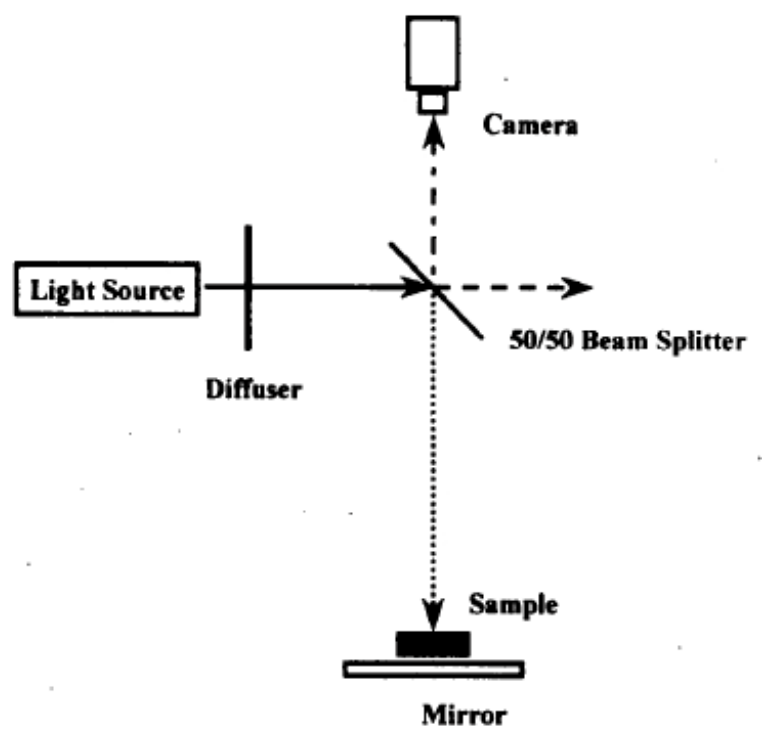

Figure 13: Schematic of the Directional Lighting System Used to Capture Nonwoven Images (Pourdeyhimi et al., 1999)

Next, the resulting image must go through a number of processes before the ODF can be found. These processes are: relaxation, thresholding (segmentation), and possibly edge thresholding and skeletonising.

Relaxation refers to the use of a local contrast enhancement procedure to improve the results prior to thresholding. This process regroups the grey level values in a histogram. This procedure is performed because performing thresholding alone may result in loss of detail in smaller fibre segments.

Thresholding, also known as segmentation, is performed to separate the background from the foreground. In this step, the grey values are classified as light or dark based on a threshold or cut-off value. Usually, the mean intensity is used as the threshold value. However, this is only acceptable with bimodal histograms, indicating a clear distinction between light and dark. This step is important and has a direct influence on the final results.

Sometimes, edge thresholding is performed on an image. This procedure is performed when there is not sufficient separation between foreground and background and normal thresholding does not suffice. As shown in Figure 14, these nonwovens usually have large uniform grey areas. This procedure finds the edges (fibres). 


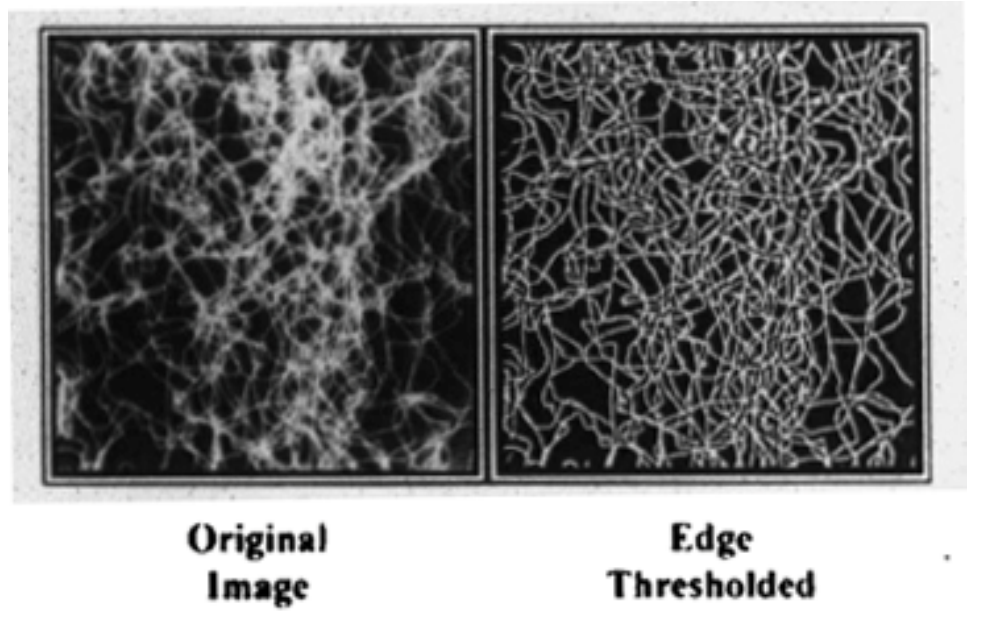

Figure 14: Edge Thresholded Image (Pourdeyhimi, Dent, Jerbi, Tanaka, \& Deshpande, 1999)

Also, skeletonising, known as thinning, is used on an image. In this process, the backbone of the object is extracted. A "morphological filter" is used to remove small isolated black regions before branching and pruning as shown in Figure 15.
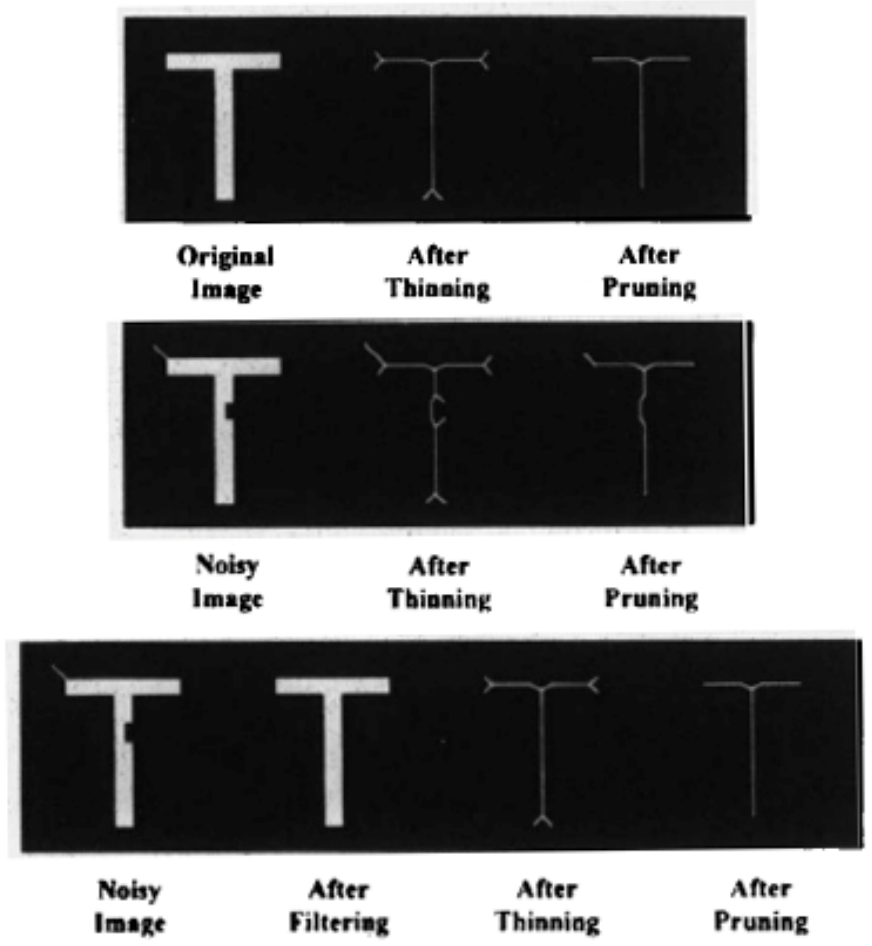

Figure 15: Thinning and Pruning Examples: (a) Top: Ideal Image with Minimal Branching, (b) Middle: Noisy Image Resulting in Excessive Branching, (c) Bottom: Noisy Image Filtered to Minimize Branching (Pourdeyhimi et al., 1999) 


\subsubsection{Fibre Orientation, "Fisheyes" and Other Defects}

In addition to the fibre orientation, which is often used as a measure of quality, fabrics may have other aspects that influence its quality that may be considered a defect. The first paper that will be discussed involves Non-Crimp Fabrics (NCF) and the second paper involves normal fabrics used in the textile industry.

Schneider, Edelmann, and Tiltmann (2004) discussed the need of an objective and automatic way of performing quality control for the large array of sophisticated NCF textiles created in the past two decades. Quality control was previously based on visual inspection and the mechanical testing of the final composite laminate. Visual inspection is time consuming and mechanical testing is expensive. Therefore, a method that can speed up the production process and find suitable textile manufacturing configurations improving the final laminate performance is very much desired. The mechanical performance of a laminate is determined by the type of fibre and resin used. Also, it is determined by the textile quality: fibre orientation, gaps, fuzzballs, missing sewing yarns/sewing thread, "fisheyes" or voids caused by the sewing thread, etc. as shown in Figure 16. Fibre orientation is determined during the textile manufacturing process and also the laminate manufacturing process.

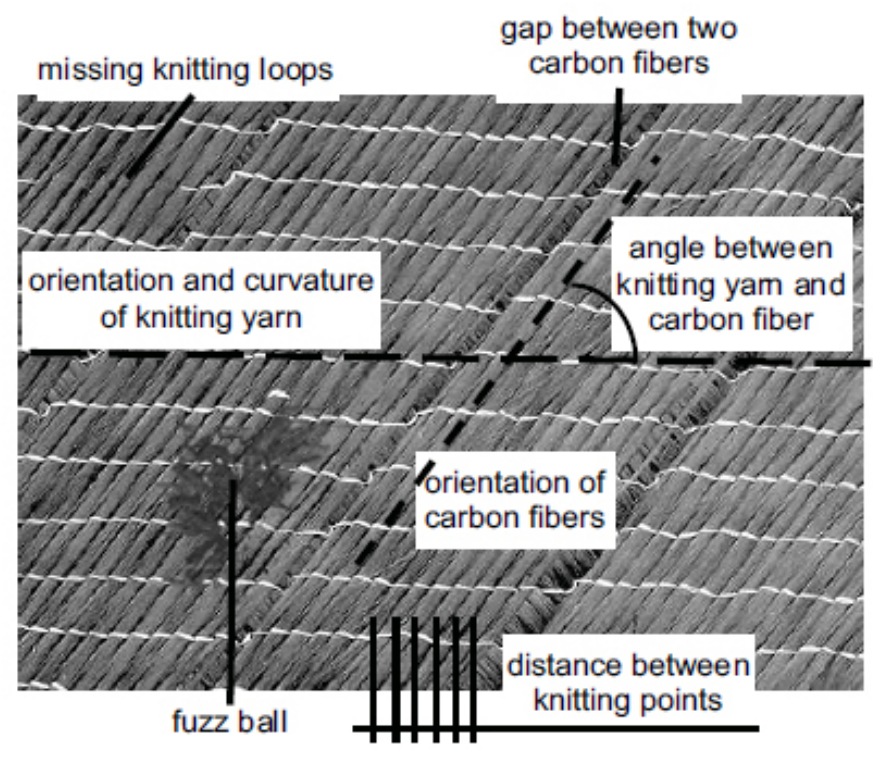

Figure 16: NCF with Exemplary Defects (Schneider et al., 2004)

The proposed method to deal with the aforementioned problem involves taking digital images of the NCF's and using software, analySIS, to analyse the images, and using Excel VBA to further analyse the results. Schneider et al. (2004) took digitised images of the textiles using a 
flatbed scanner. In particular, a $10 \times 10 \mathrm{~cm}$ size of the textile was scanned under a resolution of $720 \mathrm{dpi}$ and saved as an 8 bit grey scale tiff file. This size and resolution was chosen as a compromise between file size and computer processing speed.

Of particular interest of this study has been on the topic of "fisheyes," which are voids in the textile at the location where the sewing yarn/thread is inserted into the textile. This is done to hold more than one layer of fabric, oriented in different directions, together. As a result of these "fisheyes," the carbon fibres are no longer straight and the voids become unreinforced resin rich areas in the laminate. These areas may cause final failure of the laminate as depicted in Figure 17.

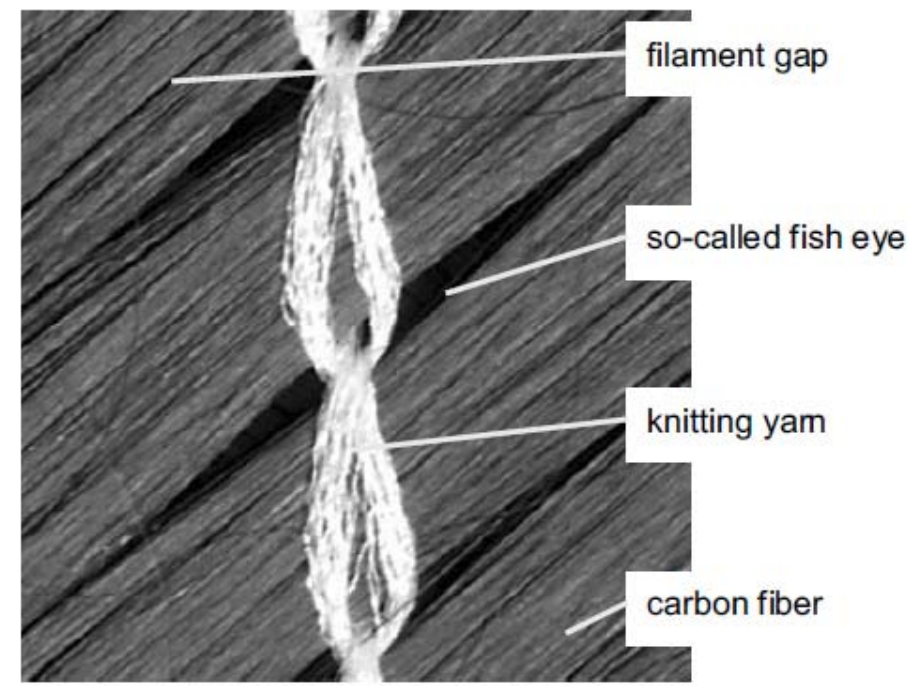

Figure 17: Detailed Picture of NCF (Schneider et al., 2004)

The analySIS software analyses the digital image of the textile to count and to give measurements of the particles in the image. A specific code written in the analySIS software performs this task. Figure 18 shows an example of an analysed image. Furthermore, these results are further analysed using Excel VBA to categorise the particles in the categories of "filament gap," fisheyes," "gap," and "knitting yarn." Also, statistics such as the count of each particle, mean and maximum length and width in millimetres, orientation in degrees, and area in $\mathrm{mm}^{2}$ are calculated and tabulated in a table as shown in Figure 19. 


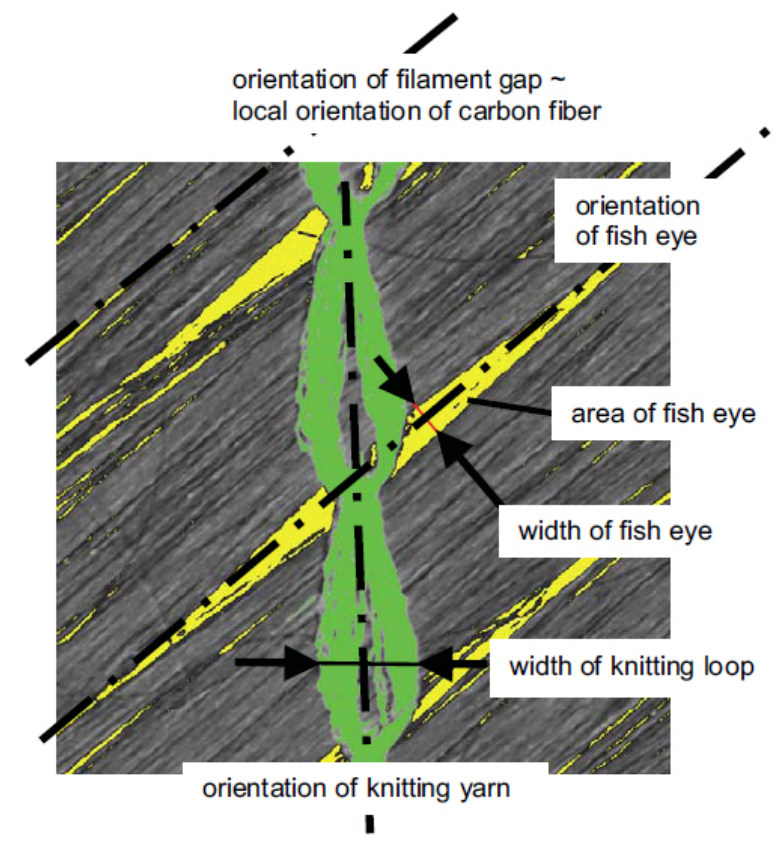

Figure 18: Detail of Analysed NCF (Schneider et al., 2004)

\begin{tabular}{|c|c|c|c|c|c|c|c|c|c|c|c|c|c|c|c|}
\hline & \multicolumn{3}{|c|}{\begin{tabular}{|c|} 
filament gaps \\
(carbon fiber orient.)
\end{tabular}} & \multicolumn{3}{|c|}{ fish eyes } & \multicolumn{3}{|c|}{$\begin{array}{c}\text { gap } \\
\text { (width }>2 \mathrm{~mm} \text { ) }\end{array}$} & \multicolumn{3}{|c|}{$\begin{array}{c}\text { knitting } \\
\text { parameter }\end{array}$} & \multirow{2}{*}{$\begin{array}{l}\text { carbon } \\
\text { fiber } \\
\text { orient. vs. } \\
\text { knitting } \\
\text { yarn } \\
\text { orient. }\end{array}$} \\
\hline & & & $\frac{\mathbb{2}}{\frac{\pi}{2}}$ & $\frac{5}{\frac{5}{3}}$ & 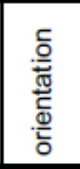 & 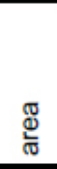 & $\frac{5}{\frac{5}{3}}$ & 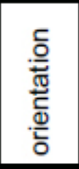 & $\frac{\mathbb{W}}{\mathscr{\Phi}}$ & $\frac{5}{3}$ & 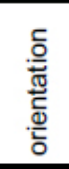 & 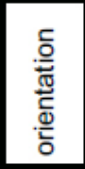 & 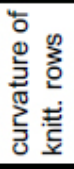 & 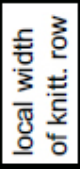 & \\
\hline \multirow{3}{*}{ 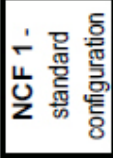 } & \multirow{3}{*}{$\begin{array}{c}\text { statistics of all } \\
\text { detected } \\
\text { partides }\end{array}$} & $\begin{array}{c}\text { number of } \\
\text { detected part. }\end{array}$ & \multicolumn{3}{|c|}{3659} & \multicolumn{3}{|c|}{1894} & \multicolumn{3}{|c|}{0} & \multicolumn{3}{|c|}{25} & \\
\hline & & mean value & 0,03 & 0,1 & 12,9 & 0,4 & 0,2 & 14,1 & & & & 135,7 & 1,4 & 0,9 & 47,8 \\
\hline & & maximum & 0,30 & 0,1 & & 1,7 & 0,4 & & & & & & 1,7 & 1,0 & \\
\hline \multirow{3}{*}{ 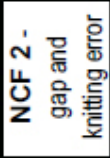 } & \multirow{3}{*}{$\begin{array}{c}\text { statistics of all } \\
\text { detected } \\
\text { partides }\end{array}$} & $\begin{array}{c}\text { number of } \\
\text { detected part. }\end{array}$ & \multicolumn{3}{|c|}{1775} & \multicolumn{3}{|c|}{1757} & \multicolumn{3}{|c|}{14} & \multicolumn{3}{|c|}{37} & \\
\hline & & mean value & 0,03 & 0,1 & 149,0 & 1,4 & 0,5 & 163,4 & 15,7 & 2,9 & 27,3 & 47,2 & 1,5 & 0,9 & 47,2 \\
\hline & & maximum & 0,30 & 0,1 & & 3,3 & 0,7 & & 18,4 & 3,5 & & & 3,3 & 3,2 & \\
\hline \multirow{3}{*}{ 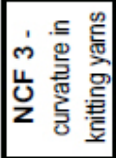 } & \multirow{3}{*}{$\begin{array}{c}\text { statistics of all } \\
\text { detected } \\
\text { partides }\end{array}$} & $\begin{array}{c}\text { number of } \\
\text { detected part. }\end{array}$ & \multicolumn{3}{|c|}{2899} & \multicolumn{3}{|c|}{2139} & \multicolumn{3}{|c|}{0} & \multicolumn{3}{|c|}{27} & \\
\hline & & mean value & 0,03 & 0,1 & 30,7 & 0,4 & 0,2 & 37,4 & & & & 136,0 & 3,1 & 0,8 & 44,5 \\
\hline & & maximum & 0,40 & 0,1 & & 3,4 & 0,7 & & & & & & 6,5 & 1,0 & \\
\hline
\end{tabular}

Figure 19: Summary of Final Results (Schneider et al., 2004)

Hu and Xin (2004) had the same problem that Schneider et al. (2004) had with an objective and automated way of performing quality control on fabrics. Hu and Xin's objective was slightly different in which they were trying to analyse pilling, polar fleece, and wrinkling of fabrics. In the past, fabrics were assigned a grade based on visual inspection, given that there were templates of the different possible conditions of the fabric. For these different kinds of defects, they proposed three different ways to quantify them. 
For pilling, a CCD camera was used to capture images of the fabric. However, this presented a problem with patterned fabric. The solution to this problem was using a laser scanner and structural lighting to create a three dimensional surface profile. A two parameter Gaussian function was used to model a pill, a template for pill detection. The six step process is: image acquisition, template training, template matching, image segmentation, feature extraction, and grade rating. Template training involves finding the fitted Gaussian function, "the template." Template matching involves comparing the template with the image. Image segmentation produces a binary image highlighting the pills. In feature extraction, pill properties are found by evaluating: pill number, area (size), contrast, and density. Finally, a grade rating is given to the pilled fabric. The objective rating was compared to the subjective rating there was a good correlation.

For polar fleece fabrics, the texture comes in the form of being dense or being "fluffy." Therefore, fractal analysis was used to perform dilation and erosion operations on fabric images because it has been found that the grade rating is correlated to the fractal dimension. If there is more than one value for fractal dimension, the extended morphological fractal analysis is used. The Bayes distance function is used to convert from the fractal dimension to an objective grade for the fabric. It has been found that there is a good correlation between the objective and subjective grades.

For wrinkling, the crease was considered to be three dimensional. A laser probe was used to scan the surface of the fabric. The fabric was illuminated at four different directions. Then, the acquired data was used to reconstruct the actual three dimensional surface of the fabric. Two functions, $P$ and $Q$, were defined and used for feature extraction and the wrinkling status of the fabric. Here, $P$ is the wrinkling in the $x$ direction and $Q$ is the wrinkling in the $y$ direction. Therefore, $P+Q$ is the wrinkling in both directions. It has been found that there is a high correlation between $P+Q$ and the subjective rating (Hu \& Xin, 2004).

\subsubsection{Non-destructive Inspection}

Non-Destructive Testing (NDT) performed prior to entry into service and post-production of a part, is an important step. When the composite part has entered into service, such as use in an aircraft, Non-Destructive Testing comes in the form of Non-Destructive Inspection (NDI). Armstrong and Barrett (1998) described the different types of damage and NDI techniques. At 
the manufacturing stage, before the aircraft enters service, the aircraft is inspected. Voids in the matrix, broken or buckled fibres, incorrect fibre type, incorrect fibre orientation, and disbonding due to contamination are searched. Possible damages that can occur are disbonding, delamination, impact, cracking, heat, chemical, and moisture ingress. These damages would need to be mapped and cut away.

Table 1 is a rough guide for which NDI method can be used for which defect. Some defects may not be visible. Therefore, it may not be possible to determine which method to use. Out of all of these methods, the ultrasonic inspection method covers the most ground and is the best solution listed.

\begin{tabular}{|c|c|c|c|c|c|c|c|c|}
\hline \multirow[b]{2}{*}{ Service Defects } & \multicolumn{8}{|c|}{ Inspection Method } \\
\hline & Visual & $\begin{array}{l}\text { Tap } \\
\text { Test }\end{array}$ & $\begin{array}{l}\text { Acoustic } \\
\text { Impedance }\end{array}$ & Ultrasonic & Thermography & $\begin{array}{l}\text { X- } \\
\text { Ray }\end{array}$ & Hologram & $\begin{array}{l}\text { Eddy } \\
\text { Current }\end{array}$ \\
\hline Disbond & $\mathrm{x}$ & $\mathrm{x}$ & $\mathrm{x}$ & $\mathrm{x}$ & $\mathrm{x}$ & & $\mathrm{x}$ & \\
\hline Delamination & $\mathrm{x}$ & $\mathrm{x}$ & $\mathrm{x}$ & $\mathrm{x}$ & $\mathrm{x}$ & & $\mathrm{x}$ & \\
\hline Impact & $\mathrm{x}$ & & & $\mathrm{x}$ & & $\mathrm{x}$ & $\mathrm{x}$ & \\
\hline Cracks & $\mathrm{x}$ & & & $\mathrm{x}$ & & $\mathrm{x}$ & & $\mathrm{x}$ \\
\hline Heat Damage & $\mathrm{x}$ & $\mathrm{x}$ & & $\mathrm{x}$ & & & $\mathrm{x}$ & \\
\hline Chemical Damage & $\mathrm{x}$ & & & & & & & \\
\hline Moisture & & & & $\mathrm{x}$ & $\mathrm{x}$ & $\mathrm{x}$ & & \\
\hline
\end{tabular}

Table 1: Defects and Appropriate NDI Methods (Armstrong \& Barrett, 1998)

The tap test is only used for outer surface inspection. Other methods such as the acoustic impedance method should be used for inspecting the underlying layers, as well as x-ray, and thermography. X-ray and thermography is particularly suited for the detection of moisture. Penetrant-enhanced x-radiography is used when the surface crack is visible. This process uses a penetrant to reach the delaminated layer. It is toxic and can cause contamination. Therefore, it can only be used in a laboratory.

In addition to the NDI methods listed in Table 1, there are some new developments. One of them is the Electric Resistance Change Method (ERCM). This method is suited for high accuracy estimation of delaminations. It is also used for estimating the strain, the delamination crack, matrix crack monitoring, defects that are difficult to visually detect. However, this method requires mounting many electrodes. This method works on the basis that carbon fibres have high electrical conductivity and that epoxy matrix has insulation resistance. This method is ideal because electrical conductance in fibre direction is very high. Fibre breakage causes decrease in electrical conductance in cross sectional area and matrix cracking results in increase in electrical resistance (Todoroki, Hirai, \& Matsuzaki, 2008). 
Another NDT method related to ERCM is the two-stage electric potential change method (EPCM). This method is for large structures or parts that require rapid monitoring. Fewer electrodes need to be mounted. The basis of this method is on the fact that carbon fibres have piezoresistivity. Under strain, electrical resistance increases proportionally and electric potential changes (Todoroki A., 2008).

Fiber Bragg Gratings can be used for NDT. They are sensor fibres embedded into the fabrication of the composite or adhered to the surface of composite. They reflect a specific wavelength, $810-840 \mathrm{~nm}$, around $1 \%$ of light and are read by illuminating gratings with broadband light source. Analysing the reflected radiation with imaging spectrometer reveals damage information (Brönnimann, Nellen, \& Sennhauser, 2000).

Vibrothermography is performed through vibration through mechanical shaker. Mechanical energy is converted to thermal energy due to acoustic damping. Defects have stronger damping, therefore, higher temperature generation. An infrared camera and software can be used to pick-up defects (Rantala, Wu, \& Busse, 1995).

\subsubsection{Destructive Testing}

There are different ways to perform destructive testing. They all use a sample of the cured laminate to perform tests by which needs to be cut or destroyed in other ways to obtain results. Two types are discussed here: using optical microscopy to find the fibre orientation and tensile/compression testing.

\subsubsection{Optical Microscopy for Fibre Orientation}

Yugartis (1987) described a way to obtain fibre orientation through optical microscopy. In this method, fibre misalignments between $\pm 10^{\circ}$ (suited for small misalignments) with estimated resolution of $\pm 0.25^{\circ}$ were obtained. In-plane and out-of-plane misalignments were found for prepregs. Previous to this study, analysis was performed on short fibre composites, with direct measurements of fibre angle with a protractor on micrographs or angular reticule in microscopes. In order for this method to be successful, sample sections showing sufficient contrast between fibre and matrix need to be prepared. This method was only useful for large angle misorientations. 
Recall that local initial fibre misalignment causes kink band formation (Argon, 1972) and angle of kinked fibres $(\alpha)$ related to angle of kink band $(\beta), \alpha=2 \beta$. This means that change from $1^{\circ}$ to $2^{\circ}$ results in a $50 \%$ drop in strength since the strength of the laminate is highest when the load is in the direction of the fibre.

A sample infused in resin is sectioned flat with swing-grinder (estimate $\emptyset_{\mathrm{pc}}$ ). Then, metallographic polishing is performed, and the sample photographed with reflected light microscopy.

From Figure 20, it can be shown that:

$$
\sin \omega=\frac{d}{l}
$$

Where $d$ is the cylinder diameter

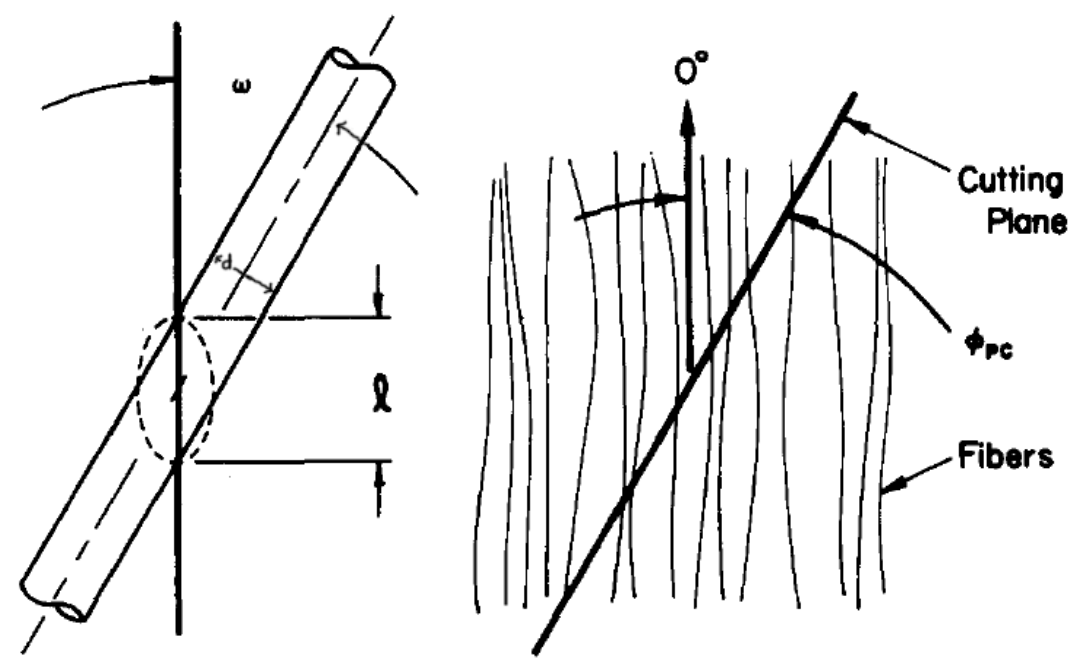

Figure 20: Ellipse resulting from the plane section of a right circular cylinder (left); Definition of the plane-cut angle $\phi_{P C}$ (Yurgartis, 1987)

The distribution of $l_{i}$ should give a distribution of $\omega_{i}$ and the assumptions are that short section of fibre is approximately straight and all fibres have same circular diameter.

The process involves sectioning and polishing composite laminate sample, measuring distribution of $l_{i}$ to calculate $\omega$, transforming $\omega_{i}$ to $\varnothing_{i}$, finding the distribution of $\varnothing$, the angle of the fibre with respect with the $0^{\circ}$ direction, mean fibre direction. 
The value, $\omega_{i}$, is the angle of the $i^{t h}$ fibre segment with respect to the sectioning plane. It is the angle of the fibre with respect with the mean fibre angle. Transforming $\omega_{i}$ to $\varnothing_{i}$ will give the angle with respect with the $0^{\circ}$ direction.

$$
\varnothing=\omega-\emptyset_{p c}
$$

Where $\emptyset_{p c}$ is the plane-cut angle, the mean of $f(\omega)$, therefore theoretically, $\emptyset_{p c}=\bar{\omega}$.

Some attention needs to be paid to $\omega$ because sensitivity of $\ell$ to $\omega$ is greatest when $\omega$ is small. As $\omega$ approaches $0, \ell$ becomes large and makes $\ell$ difficult to measure. Also, when $\omega<\emptyset_{p c}$ the measured distribution folds back upon itself. According to equation 2.16 this results in a negative angle. Therefore, it is assumed that true distribution is symmetric. In addition, small $\omega$ is less sensitive to variations in $d$. With $\omega \approx 0, \ell$ is probably improperly measured because $\ell$ is large because measured fibre segments cannot be assumed to be straight due to curvature. The value, $\emptyset_{p c}$, needs to be carefully chosen.

Next, $f(\varnothing)$ is found from $f(\omega)$ at $\omega \geq \omega_{\text {median }}$ with transformation $\emptyset=\omega-\omega_{\text {median }}$. Here, $\bar{\omega}$ is changed to $\emptyset_{p c}=\omega_{\text {median }}$ since $\omega_{\text {median }}$ has been shown to be more accurately obtained. The disadvantage in doing this is discarding data and possibly reducing angular resolution due to reduced sensitivity at large angles.

The value, $\varnothing$, refers to in-plane misalignment angle and $\theta$ refers to out-of-plane misalignment angle as shown in Figure 21. The angle $\theta$ is measured independently of $\varnothing$, by a sectioning plane taken at $90^{\circ}$ from each other. If $\varnothing$ and $\theta$ are statistically independent, no correlation between each other, then, $f(\emptyset, \theta)=f(\varnothing) f(\theta)$.

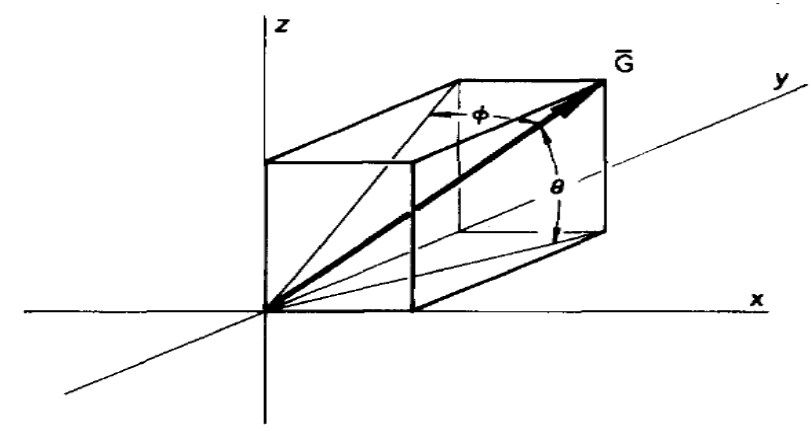

Figure 21: Definition of the in-plane and out-of-plane angles, $\varnothing$ and $\theta$, respectively. The fibre axis is represented by vector $\bar{G}$ (Yurgartis, 1987) 
The fallback to this study is that the distribution of fibre diameters uncertain and it has been found that lamination can change the fibre misalignment distribution.

In another study (Creighton, Sutcliffe, \& Clyne, 2001), the two dimensional fibre alignment of a laminate has been found through a technique called Multiple Field Image Analysis (MFIA).

The relationship between compressive stress and fibre angle can be summarised in equation 2.17 defined by Budiansky and Fleck (1993).

$$
\sigma_{k}=\left(\frac{1}{G_{12 c}}+\frac{\emptyset_{0}}{\tau_{12}}\right)^{-1}
$$

The value, $\sigma_{k}$, is applied compressive stress; $\emptyset_{0}$ is sum of original misorientation; $\tau_{12}$ is shear stress; $G_{12 c}$ is shear modulus of the composite.

The MFIA technique uses optical micrograph of pultruded composite and prepreg. Creighton et al. (2001) indicated that the prepreg material is not suitable for Yugartis technique since it is difficult to choose representative points for sectioning and it is difficult ensuring universal reference direction over large area of specimen.

There was an attempt to obtain the three dimensional orientation distribution. However, it became too cumbersome and was only applicable for small number of fibres at few locations.

In MFIA technique, the fibre alignment angles found for each domain, automated with computer program. The light intensity, $I$, changes with the different orientations of the fibre at the surface of the sample as shown in Figure 22. As depicted in Figure 23, the orientation based on a pixel array. The grid points within each domain are rotated to an angle $\theta$ relative to the reference direction. The corresponding contour map of measured average misalignment angle within each domain is shown in Figure 24.

The value, $\delta$, is the intensity variation parameter. Based on each $\theta$, the following equation results:

$$
\delta_{\theta}=\frac{1}{2 m+1} \sum_{i=-m}^{i=+m}\left|I\left(P_{0}\right)-I\left(P_{I}\right)\right|
$$



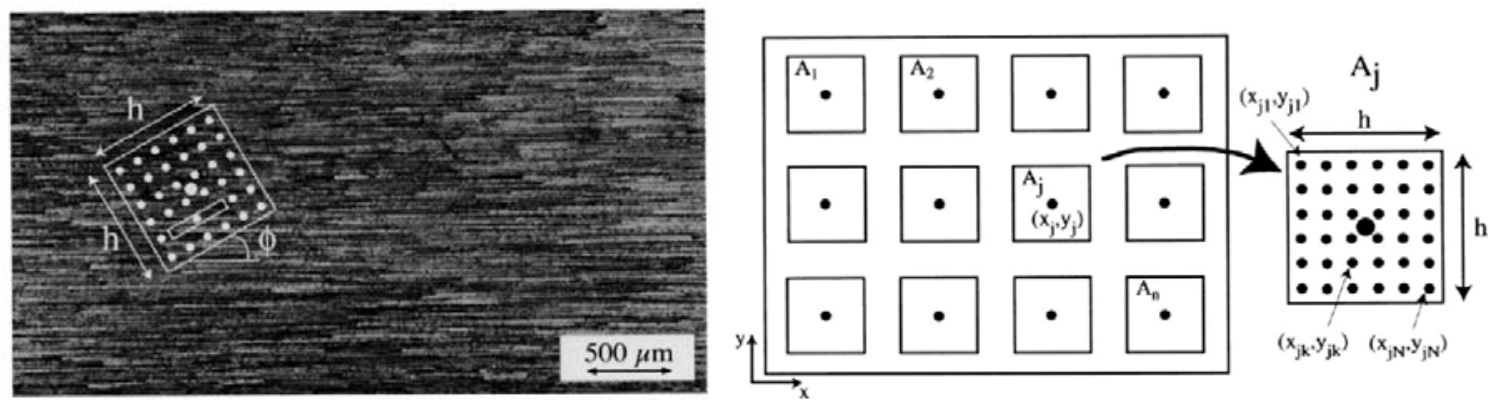

Figure 22: Optical micrograph of a pultruded composite, section cut in parallel to pultrusion direction, with a domain and pixel array highlighted (left); Schematic depiction of domains $\left(A_{j}\right.$ centred at $\left.x_{j}, y_{j}\right)$ within which the average fibre orientation $\varnothing$ is to be measured (right) (Creighton et al., 2001)

1-D pixel array of length $L$

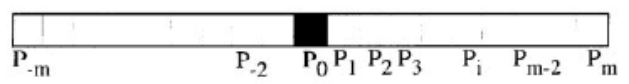

Pixel array superimposed on image at an angle $\theta$ to reference direction

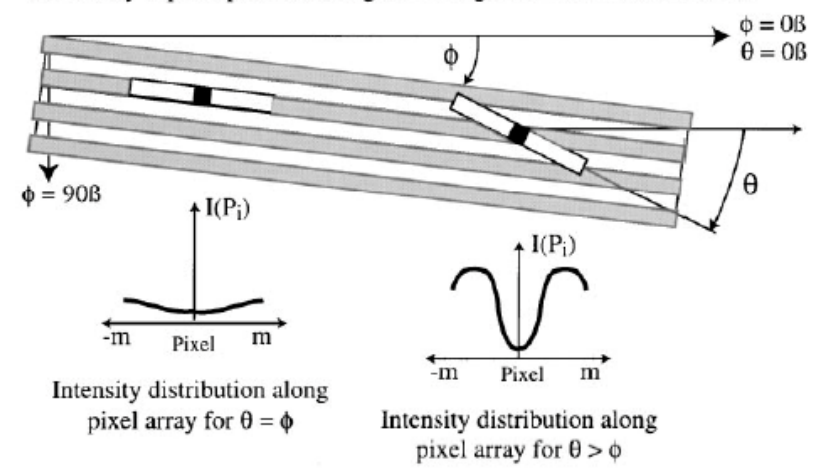

Figure 23: Schematic depiction of a 1D array of pixels within an image, inclined at angle $\theta$ to the reference direction, showing typical intensity distributions along the length of the array, for two values of $\theta$, one which is equal to and the other greater than the fibre misalignment angle $\sigma$ (Creighton et al., 2001)

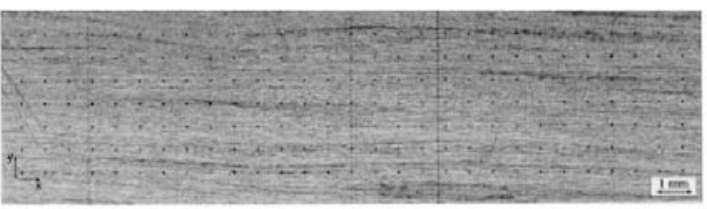

(a)

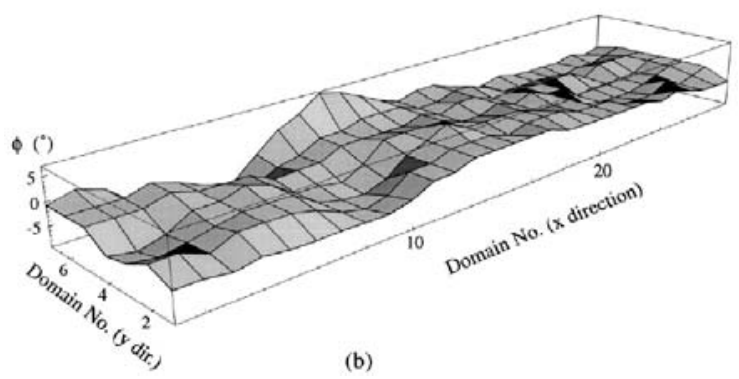

Figure 24: a) Optical micrograph showing the free surface of prepregged sheet material, with the centroid of each measurement domain marked by a black dot, b) Corresponding contour map of measured average misalignment angle within each domain, with each grid intersection point corresponding to one of the black dots on the micrograph (Creighton et al., 2001) 
The intensities are sampled at $2 \mathrm{~m}+1$ regularly spaced points along length of array, a process which measures the variation of intensity along the array. Then, the average is found:

$$
\delta_{\theta j}=\frac{1}{N} \sum_{k=1}^{k=N} \delta_{\theta}
$$

Then, the plot $\delta_{\theta j}$ against $\theta$ is created. The minimum of the plot for the corresponding $\theta$ is the $ø$ for the domain is found, through cubic interpolation algorithm.

To implement the MFIA method, the length of an array needs to be specified. This value needs to be greater than a characteristic feature length. Also, the size of domain needs to be specified to a value that is not too small or unreliable results will be the outcome.

\subsubsection{Compression and Tensile Testing}

In compression and tension testing, the sample is placed in a machine that loads the sample in compression or tension until failure. The amount of compression stress that a sample can tolerate until failure is called the compression strength. Likewise, for tension, it is called the tensile strength. Compression testing is the most difficult of the intrinsic material properties to measure since "slight specimen geometric variations result in eccentricity of the applied load, thereby enhancing the opportunity for failure to occur due to geometric instability" (Whitney, Daniel, \& Pipes, 1984). Complicated loading fixtures and specimen configurations are required.

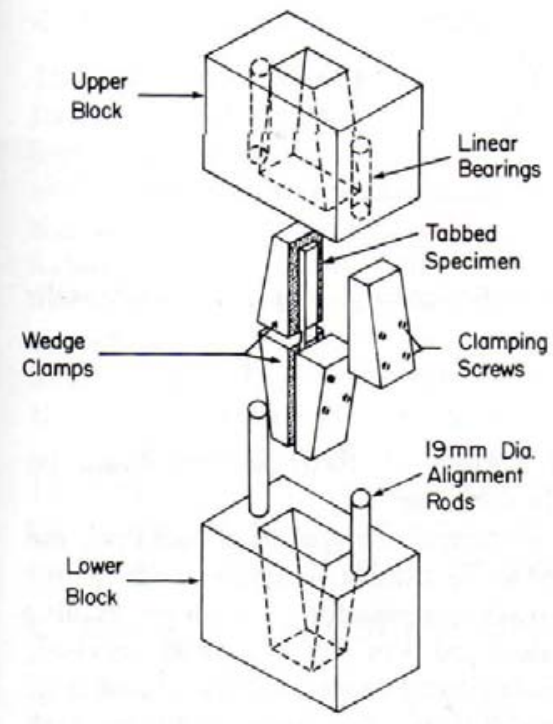

Figure 25: IITRI compression test fixture (Mallick, 1997) 
There can be a large variability in results, which indicates a need to improve test methodology, especially the mode and location of failure, related to stress concentrations in specimens (Spragg \& Drzal, 1996).

The tensile test is performed similarly to the compression test. "The tensile test is performed in order to determine uniaxial tensile strength, Young's modulus and Poisson's ratio." (Whitney, Daniel, \& Pipes, 1984)

\subsection{PREDICTION OF FAILURE}

Naik and Kumar (1999) performed a study that looked at the different models used for the prediction of compression strength. The studies have been based on UD composites and there are many models, many controversies. As discussed in Section 2.1 in Chapter 2, micro-buckling and kinking go hand in hand. Fibre failure due to micro-buckling results in kinking. Models based on micro-buckling include: Rosen Model, Lo-Chim Model, Xu-Reifsnider Model, and the Lagoudas-Tadjbakhsh-Fares Model. Models based on kinking are: Budiansky Model and Lagoudas-Saleh Model. In the study by Naik and Kumar (1999), these models were evaluated and compared to data obtained from the literature. Some models had parameters that were specific to lay-up technique, material defects, bonding, etc. Therefore, only the critical parameters were chosen in the evaluation. Also, in this study, only E-glass/epoxy and carbon/epoxy combinations were studies since these combinations had the most referenced compressive strengths. The result was that the Xu-Reifsnider Model and Budiansky Model predicted compression strength quite accurately.

The aforementioned models were all mathematical models. Pansart (2008) and Wietgrefe (2007) developed a way to simulate compression failure due to Fibre Micro-Buckling (FMB) or Ply Meso-buckling (PMB) with the use of Finite Element analysis with MSC Patran. FMB occurs through kink band propagation. PMB occurs through buckling of a wavy of the ply. This model is important because different prediction models have different conclusions and place different characteristics as more important. Interaction between microscopic and mesoscopic properties have not been considered in previous models.

Figure 26 visually illustrates how the results from the FMB model and the PMB model are combined to form the FMB-PMB model and to find the wavy ply compression strength. The FMB Patran model takes parameters regarding the fibre, such as different initial angle 
misalignments. A model is created and a simulation is run to determine the maximum compression strength under different initial angle misalignments. From the maximum compression stress obtained under each initial fibre angle misalignment, a sensitivity curve is plotted using MS Excel. The result of the FMB model is the combination of the sensitivity curve and the initial fibre angle misalignment distribution. The maximum of this combination is the ply compression strength with respect with the FMB model. The initial fibre angle misalignment distribution is assumed to be Gaussian. Given that initial fibre angle misalignment angles have been specified, the standard deviation also needs to be specified. The PMB Patran model takes parameters regarding the ply, such as ply waviness morphology. A model is created and a simulation is run to determine the ply instability stress and the stresses in the ply. Then, these results are combined with the results from the FMB model to determine the wavy ply compression strength. The following paragraphs describe these steps mathematically.

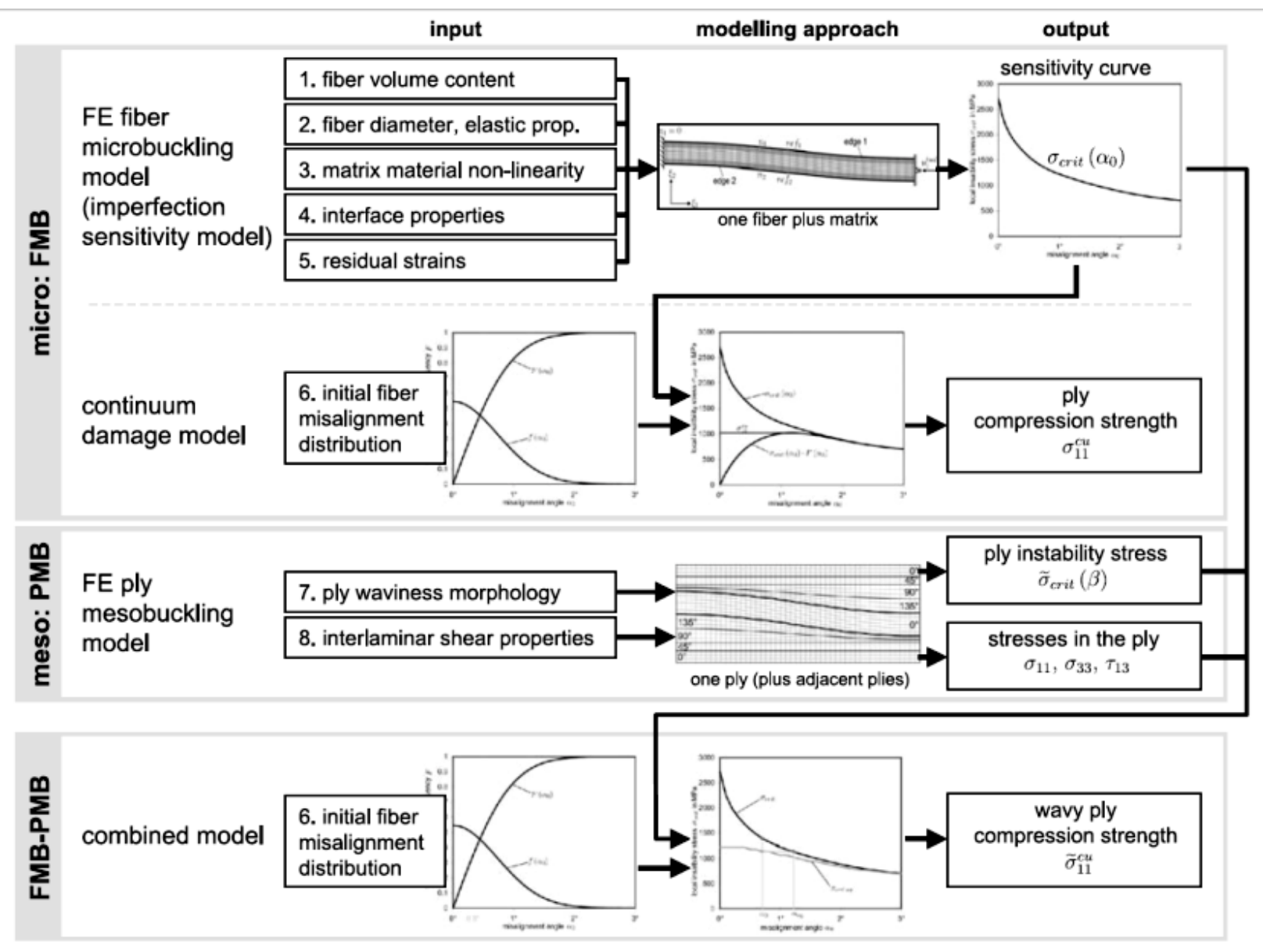

Figure 26: Hierarchical structure of the FMB-PMB approach (Pansart, 2008) 
The characteristics for micro-buckling are fibre volume content, fibre bending stiffness, material non-linearity, fibre matrix interface properties, residual strains in matrix, and initial fibre misalignment. The characteristics for meso-buckling are: ply waviness, interlaminar shear properties.

The FMB is based on initial (before loading) fibre misalignment angle $\alpha_{0}$ that assumes smooth changes in fibre angle and few values greater than $6^{\circ}$. The purpose is to find composite ultimate compression strength, $\sigma_{11}^{c u}$, of a single UD ply by: 1) using material properties, find micro-buckling stress, $\sigma_{\text {crit }}$, with respect to $\alpha_{0}$ using FEM $\left(\sigma_{\text {crit }}\left(\alpha_{0}\right)\right.$ imperfection sensitivity function results) and 2) Continuum damage model by combining $\sigma_{\text {crit }}\left(\alpha_{0}\right)$ with stochastic distribution of misalignment angles.

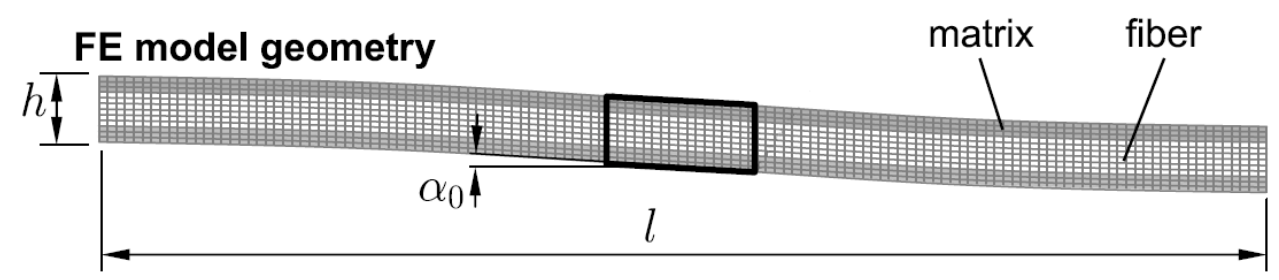

Figure 27: Idealization of the composites's microstructure and FMB FE model geometry (Pansart, 2008)

$$
\tan \alpha_{0}=A_{0} \frac{\pi}{l}
$$

From equation 2.20, it can be derived that initial fibre angle, $\alpha_{0}$, depends on model length, $\ell$, and amplitude, $A_{0}$. This assumes there are adjacent fibres on both sides (top and bottom) of the modelled fibre and only the centre part of the model is used for analysis. A FEM (Patran) is used to deal with many interacting non-linear material characteristics from embedding fibre in matrix such as: material non-linearity and rupture, fibre-matrix interface, and residual strength. In addition to all this, there is geometrical non-linearity of the fibre due to deflection before reaching critical load (failure).

The physical fibre volume content, $V_{f}$, based on hexagonal fibre packing, is not the same as the fibre packing fibre volume content in the two dimensional model, $V_{f}^{*}$, used for a three dimensional representation. 


$$
V_{f}^{*}=1.05 \sqrt{V_{f}}
$$

The FMB model is a two dimensional representation of three dimensions and the given fibre volume content (FVC) of a composite, $V_{f}$, is given for the two dimensional representation. In this study, $V_{f}$ is used. The FVC is important because it determines how the stress value is calculated in the FMB model since the result is given in force. Stress is equal to force divided by area. Model height, $\mathrm{h}$, is used as area here and the height is the fibre diameter divided by FVC.

In addition, $E_{11, f}, E_{22, f}, G_{12, f}, v_{12, f}$ are used to specify the material. $E_{11, f}$ is the largest stiffness which needs to be inclined by the initial fibre misalignment angle $\alpha_{0}$. The matrix failure is taken as an independent parameter influencing compression strength, while $E_{m}$ is the matrix modulus. During laminate production, the fibre and matrix undergo volume changes that result in residual strain. The epoxy polymer shrinks during curing and the cured matrix shrinks during cooling. The fibre-matrix interface is taken into account since premature failure can cause fibre micro-buckling and the magnitude of critical micro-buckling stress, $\sigma_{\text {crit }}$.

$$
\sigma_{c r i t}=\left[V_{f} E_{11, f}+\left(1-V_{f}\right) E_{m}\right] \cdot \frac{u_{1, \text { crit }}^{r 1}}{l}
$$

Where

$$
\begin{aligned}
& E_{11}=V_{f} E_{11, f}+\left(1-V_{f}\right) E_{m} \\
& \epsilon_{\text {crit }}=\frac{u_{1, \text { crit }}^{r 1}}{l}
\end{aligned}
$$

For $u_{1, c r i t}^{r 1}$, this is the critical displacement of node 1 (at the right of the modelled fibre, where left of the modelled fibre is assumed to be fixed) in one dimensions as shown in Figure 28.

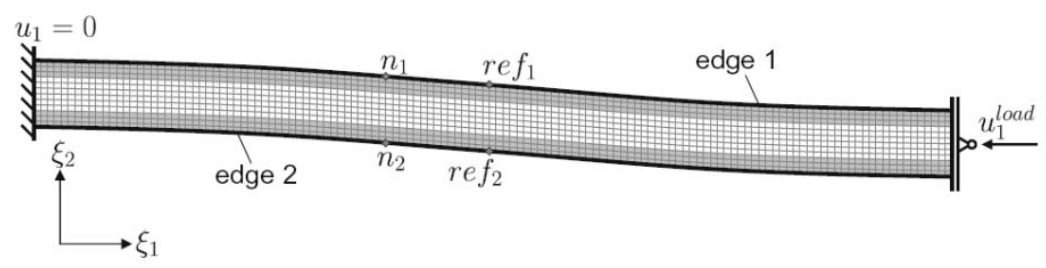

Figure 28: Loads of FMB (Pansart, 2008) 
The initial fibre angle, $\alpha_{0}$, is changed iteratively to obtain new $\sigma_{\text {crit }}$. This results in a sensitivity curve $\sigma_{\text {crit }}\left(\alpha_{0}\right)$. The sensitivity curve, $\sigma_{\text {crit }}\left(\alpha_{0}\right)$, is combined with the cumulative distribution function (CDF) of fibre misalignment $F\left(\alpha_{0}\right)$. The probability density function (PDF) is found experimentally, which is described in Section 3.1: Input Parameters and Section 3.4.1: Sample Standard deviation of Fibre In-plane (SFI) in Chapter 3.

For the FMB model, the compression strength of the ply is:

$$
\sigma_{11}^{c u}=\max _{\alpha_{0}}\left[\sigma_{c r i t}\left(\alpha_{0}\right) \cdot F\left(\alpha_{0}\right)\right]
$$

Where $\sigma_{11}^{c u}$ is positive for compression.

The current $\sigma_{11}^{c u}$ does not take into account if the ply is wavy in the $\mathrm{z}$ direction, thus the PMB model is needed. The goal of this model is: 1) to estimate load level for the ply to become instable, ply instability stress, $\tilde{\sigma}_{c r i t}$, and 2) estimate shear stresses in the ply, $\tau_{12}$.

Like the FMB model, the material properties are represented through:

$$
E_{11}=V_{f} E_{11, f}+\left(V_{f}-1\right) E_{m}
$$

This equation applies rules of mixture, where individual fibre and matrix properties are mixed together to find: $E_{22}, E_{33}, G_{12}, G_{13}, G_{23}, v_{12}, v_{13}, v_{23}$. Next, $\tilde{\sigma}_{c r i t}$ can be determined:

$$
\tilde{\sigma}_{c r i t}=E_{11} \cdot \frac{u_{x, \text { crit }}^{r 3}}{L}
$$

Where $\tilde{\epsilon}_{c r i t}=\frac{u_{x, c r i t}^{r 3}}{L}$

The critical displacement of node $\mathrm{r} 3$ in the $x$ direction is $u_{x, \text { crit }}^{r 3}$ (at the right of the modelled fibre, where left of the modelled fibre is assumed to be fixed), as depicted in Figure 29. 


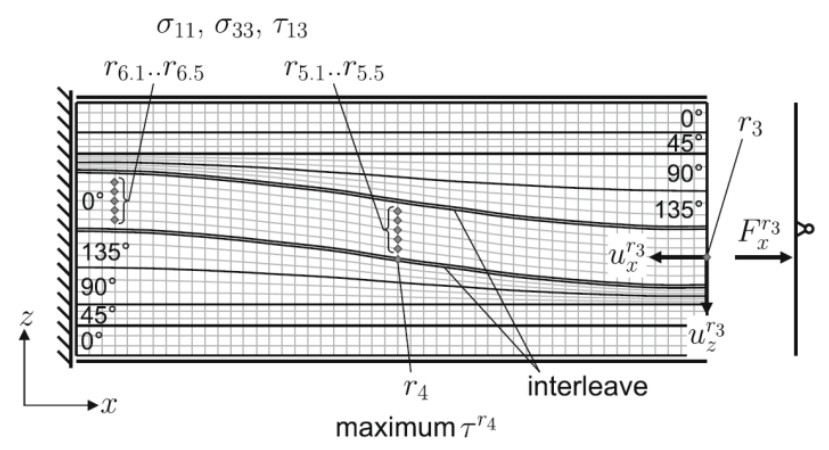

Figure 29: Finite element ply meso-buckling model (Pansart, 2008)

There is no $\sigma_{\text {crit }}\left(\beta_{0}\right)$ curve because it is assumed that the laminate will fail immediately after one $0^{\circ}$ ply has failed, instead of a progressive failure. Also, it can be noticed that $\sigma_{11}, \sigma_{33}, \tau_{13}$ found from the PMB model are not used in the PMB model to find $\tilde{\sigma}_{c r i t}$, equation 2.25 .

The Combined Micro-mesoscale Model (FMB-PMB) is performed. This step combines $\sigma_{11}^{c u}$ from FMB and $\tilde{\sigma}_{c r i t}$ from PMB to get $\tilde{\sigma}_{11}^{c u}$.

$$
\begin{aligned}
& \sigma_{11, e q}=\frac{\sigma_{11}+\sigma_{33}}{2}+\sqrt{\left(\frac{\sigma_{11}-\sigma_{33}}{2}\right)^{2}+\tau_{13}^{2}} \\
& \tan 2 \varphi=\frac{2 \tau_{13}}{\sigma_{11}-\sigma_{33}}
\end{aligned}
$$

Here, additional shear stress, $\tau_{13}$, is considered, which is shear due to inclination of $\varphi$ of fibre. It has been found that shear stresses lower compression strength. Next, $\alpha_{0}$ and $\varphi$ needs to be combined to form $\alpha_{e q}$, the equivalent initial fibre angle that can be visually represented in Figure 30.

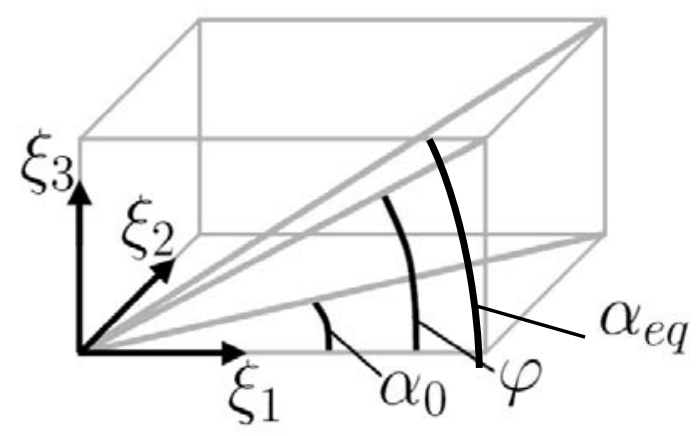

Figure 30: Addition of two angles in perpendicular planes (Pansart, 2008) 
Mathematically,

$$
\cos \alpha_{e q}=\frac{1}{\sqrt{1+\tan ^{2} \alpha_{0}+\tan ^{2} \varphi}}
$$

Then, according to equation 2.26, let $\sigma_{\text {crit,eq }}\left(\alpha_{0}\right)=\sigma_{\text {crit }}\left(\alpha_{\text {eq }}\right)$. Now, $\sigma_{\text {crit,eq }}$ is the new equivalent sensitivity curve for $\alpha_{\text {eq }}$.

Now the ultimate compression strength can be found:

$$
\tilde{\sigma}_{11}^{c u, F M B}=\max _{\alpha_{0}}\left[\sigma_{c r i t, e q}\left(\alpha_{0}\right) \cdot F\left(\alpha_{0}\right)\right]
$$

If actual local principal stress, $\sigma_{11, e q}$, compared to predicted local failure stress, $\tilde{\sigma}_{11}^{c u, F M B}$ is greater, then there is a failure. This represents an internal failure due to fibre microbuckling/kinking, which has been experimentally determined to occur first, expressed by equation 2.30, as follows:

$$
\frac{\sigma_{11, e q}}{\tilde{\sigma}_{11}^{c u, F M B}} \geq 1
$$

The compression strength of a wavy ply is estimated by:

$$
\tilde{\sigma}_{11}^{c u}=E_{11} \cdot \frac{\min \left[u_{x, c r i t}^{r 3}, u_{x, F M B}^{r 3}\right]}{L}
$$

Where, $u_{x, F M B}^{r 3}$ is the displacement for which $\frac{\sigma_{11, e q}}{\widetilde{\sigma}_{11}^{c u, F M B}} \geq 1$ is reached for the first time and $u_{x, c r i t}^{r 3}$ is the displacement for ply meso-buckling.

Since an internal failure due to fibre micro-buckling/kinking has been experimentally determined to occur first before ply mesobuckling, the compression strength prediction for the UD ply is the value of $\tilde{\sigma}_{11}^{c u, F M B}$ that satisfies equation 2.30 . 


\subsection{UNIT CELL MODEL}

Due to the "fisheyes" mentioned in Section 2.2.1.2 in Chapter 2, Heß, Roth, and Himmel (2007) developed a finite element unit cell model to estimate the elastic constants of NCF CFRP composites. Elastic constants are used in classical lamination theory to find the compressive strength of a laminate. Stitching the NCF fabric together in the thickness direction increases outof-plane properties but decreases the in-plane properties, creating NCF voids, also known as "fisheyes" or "resin pockets." In particular, the Unit Cell Model was used to determine the effect of relatively thick structural reinforcement stitching yarn used to stitch multiple layers of textile together, instead of the internal NCF stitching. Figure 31 and Figure 32 illustrate how the NCF voids are formed and the lay-up of the textiles to form a laminates used for tension and compression testing.

A numerical unit cell model was created that models the NCF voids. In this model, the yarn or sewing thread diameter, stitching pattern, cross sectional area and width of the voids, stitch spacing and pitch length, load direction across multiple layers, local fibre volume fraction (FVF) changes, as well as the number, thickness, and fibre orientation of the layers were modelled. Also, micrographs were analysed to find the mean, standard deviation, and through thickness distributions of the "fisheyes," as illustrated in Figure 33. This data was then used in the linear finite element model (ANSYS) to find the in-plane elastic constants and engineering constants, as shown in Figure 34. The model results were compared to experimental results of laminates constructed with Non-Crimp Fabrics and to analytical calculations using classical laminate theory for undisturbed fibre orientations (no voids). The analytical calculations were performed assuming that the laminate is transversely isotropic and isotropic with constrained transverse strains.

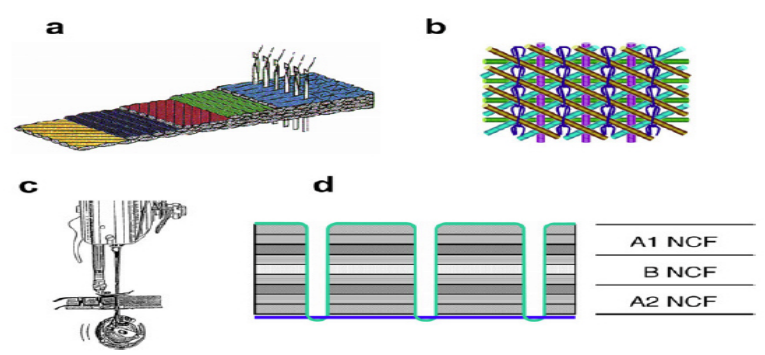

Figure 31:(a, b) NCF manufacturing process, (c, d) structural stitching of [A1-B-A2] NCF lay-up [21-23]. (a) NCF production, (b) example of finished NCF, (c) through-thickness stitching to insert structural yarn, (d) structurally stitched laminate of NCF layers (tension laminate; modified lock stitch) (Heß et al., 2007) 


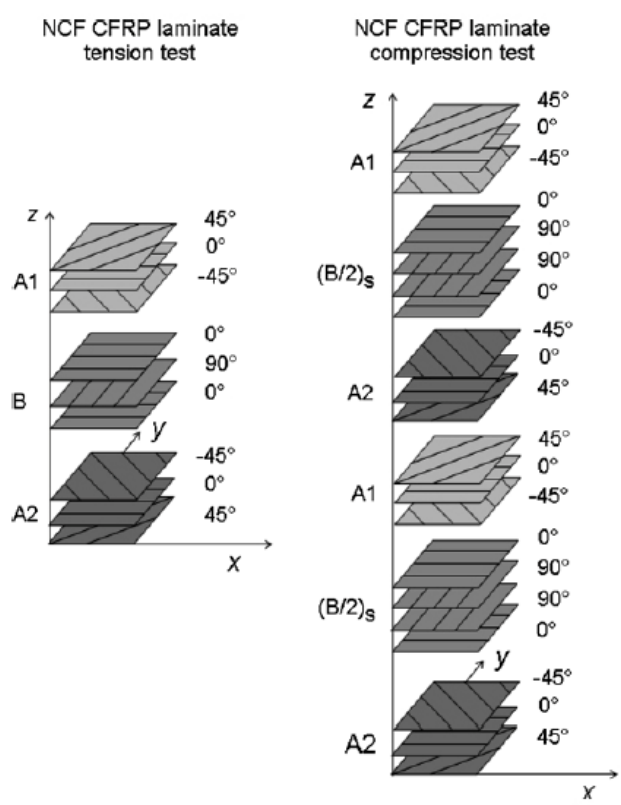

Figure 32: NCF lay-up of tension and compression CFRP laminates (Heß et al., 2007)

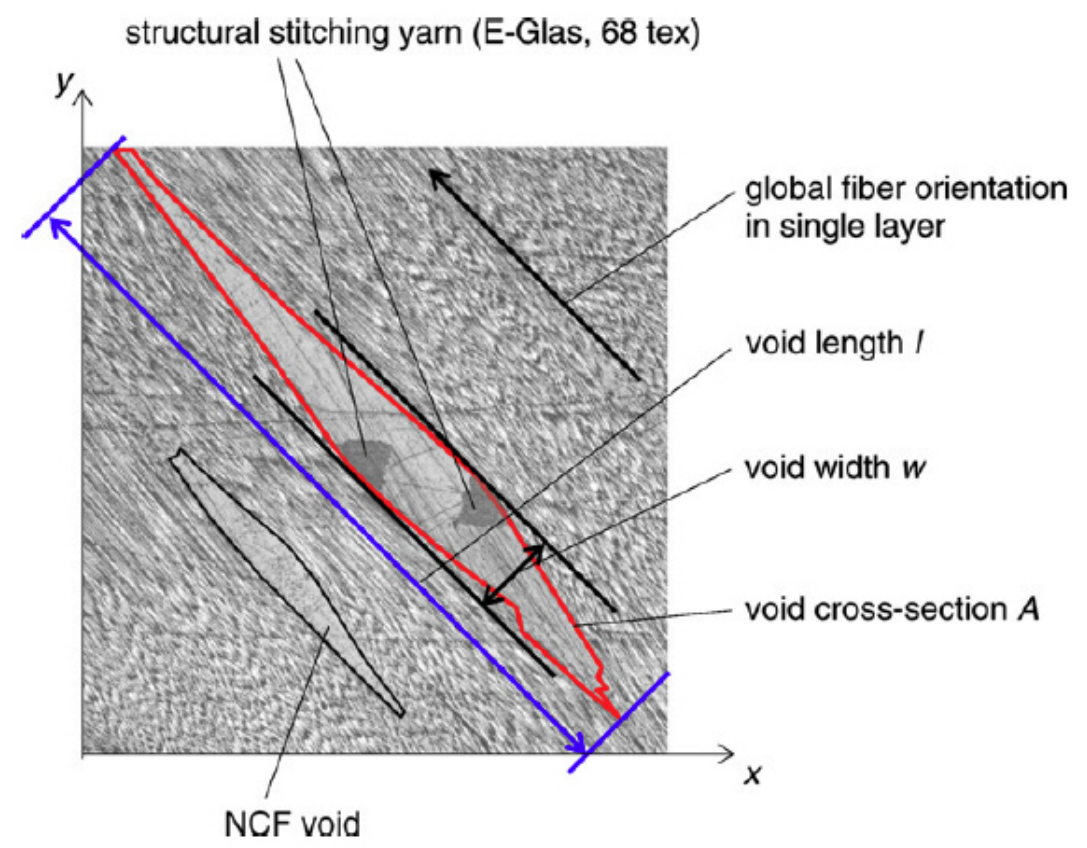

Figure 33: Micrograph through a $-45^{\circ}$ layer of a structurally stitched [A1-B-A2] CFRP laminate parallel to laminate plane (Heß et al., 2007) 


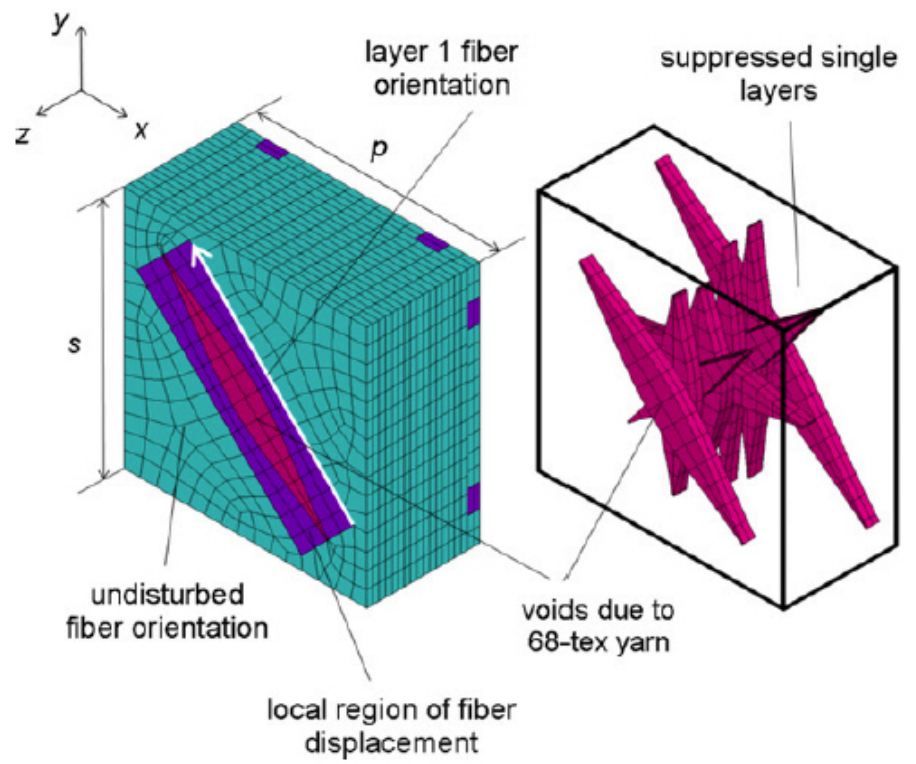

Figure 34: Unit-cell model of stitched [A1-B-A2] CFRP tension laminate (Heß et al., 2007)

Through this study, it has been found that the in-plane tensile modulus can be reduced by $29 \%$ for stitched fabrics, compared to unstitched fabrics. This can be avoided by the optimal selection of stitching parameters, such as stitch spacing and stitch length as shown in Figure 35. However, a clear tendency could not be observed for compression. Also, Young's Modulus has been reasonably estimated for the stitched laminates using the unit cell model and the mean deviation between the simulation and experimental results for tension and compression loading is $4.8 \%$ and $6.3 \%$, respectively.
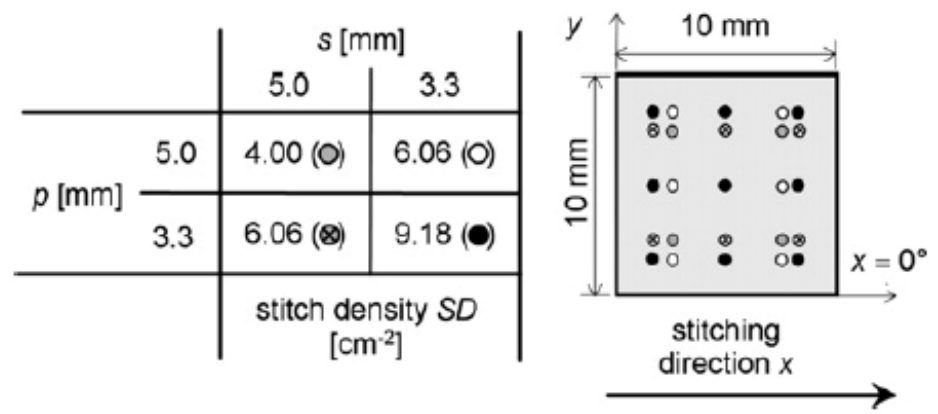

Figure 35: Structural stitching of NCF laminates: stitch patterns (spacing s and pitch length p) and stitch density SD (Heß et al., 2007) 


\subsection{APPLICATIONS OF RELIABILITY}

Reliability is understood as the probability of success, or one minus the probability of failure. Reliability in composites is most often applied as structural reliability, as detection of damage after the part has been created, such as for remaining service life of civil structures. Reliability for the use in the design phase or quality control is not frequently investigated, which is also important.

An example where reliability has been used for composites in the design phase was by Rabotnov, Tupolev, Kut'inov, Kogaev, Berezin, and Sul (1982). Here, safety factors, f, were found for composite materials.

Safety factor for composites is based on the theory of reliability, especially the probability of failure over specified period of service, $\beta$.

$\beta \propto \mathrm{f}$

$$
\mathrm{f}=\frac{P_{0} b}{P_{0} s}
$$

Where,

$P_{0} b$ median of the breaking load

$P_{0} s$ median of the operating load

Here, $f$ depends on the position and shape of distribution functions. The value, $\gamma$, is coefficient of variation of the strength properties of the composite and $\gamma=0.08$ for ordinary aircraft construction materials. Composites have larger coefficient of variation, $\gamma$. In the design stage, we use 1) additional safety factor $\mathrm{f}_{\mathrm{a}}$, which depends on coefficient of variation, $\gamma$, or 2) basic safety factor, $\mathrm{f}_{\mathrm{b}}$. Here, $\mathrm{f}_{\mathrm{a}}=1.25$ for critical or complicated structural units and elements. From Figure 36 , at $\mathrm{f}_{\mathrm{a}}=1.25, \gamma=0.15$ for $\mathrm{f}_{\mathrm{b}}=1.5$ to ensure reliability of composite structure. This is obtainable given there is quality control over raw materials and stable fabrication technology and composite coefficient of variation, $\gamma$, will not be over 0.15 , which should be close to that of metals. 

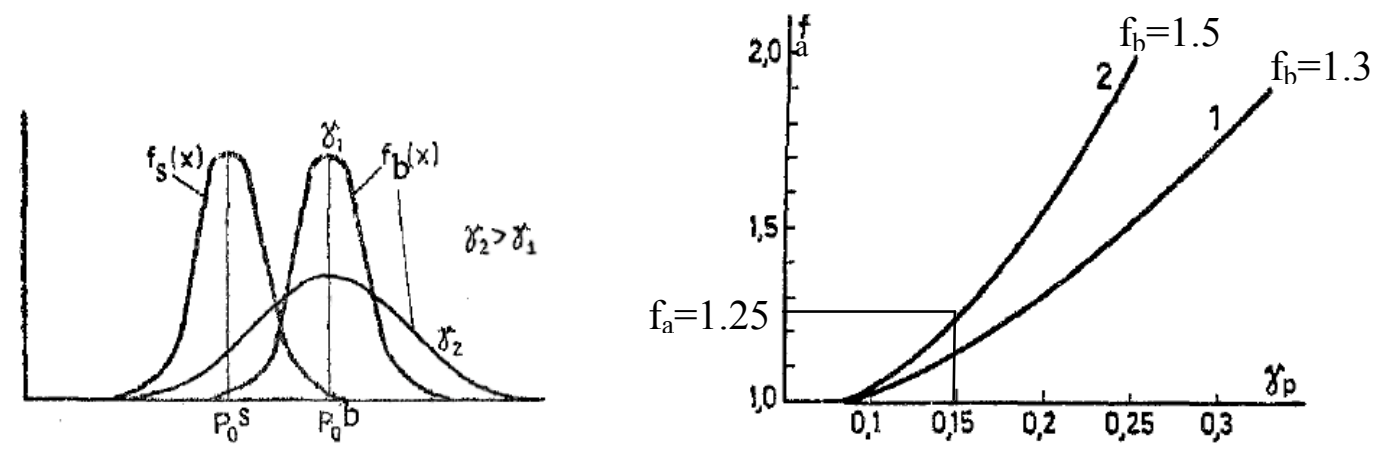

Figure 36: Probability distribution functions for breaking and operating loads (left), Additional safety factors as functions of the coefficient of variation. $f_{b}=1.3$ (1) and 1.5 (2) (right) (Rabotnov et al., 1982)

The aforementioned safety factor calculation is related to the "Strength Based Reliability and Interference Theory" by Rao (1992) describes a way to determine the reliability, R, of a component given that a probability distribution of the strength (S) and the load (L) can be found. The calculated reliability, $\mathrm{R}$, the probability of success, can have a value between 0 and 1 , where a higher number would be represent a more ideal situation.

$$
\begin{aligned}
& R=P(S>L) \\
& \qquad d R=f_{L}(l) d l \int_{l}^{\infty} f_{S}(s) d s=f_{L}(l) d l\left[1-F_{S}(l)\right]=A_{l} \cdot A_{S}
\end{aligned}
$$

Therefore,

$$
R=\int d R=\int_{-\infty}^{\infty} f_{L}(l)\left[\int_{l}^{\infty} f_{S}(s) d s\right] d l=\int_{-\infty}^{\infty} f_{L}(l)\left[1-F_{S}(l)\right] d l
$$

Strength $(S)$ and load $(L)$ need to be in the same units. Although interpretations for strength and load can vary, in most structural designs, strength can be seen as the permissible stress and load can be seen as the induced or applied stress. The probability density function, $f_{S}(s)$, corresponds to strength and $f_{L}(l)$ corresponds to load. Figure 37 gives a visual explanation of equation 2.34 . 


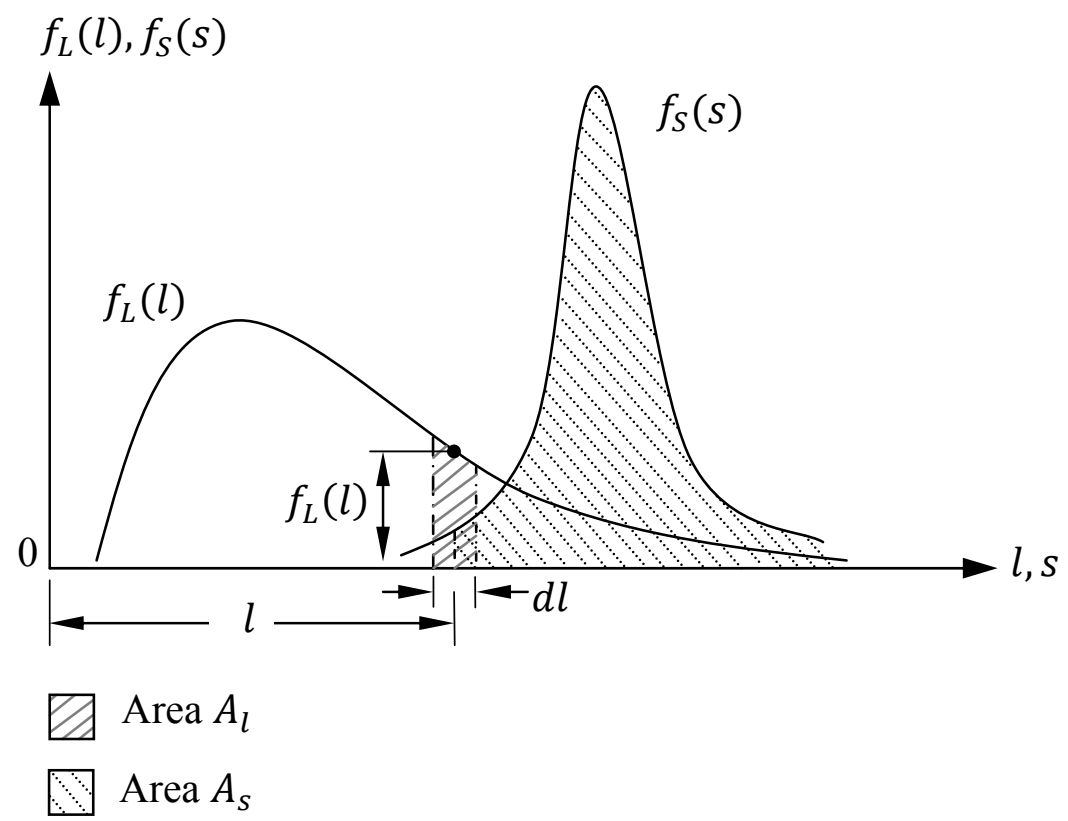

Figure 37: Definition of dR, for $S>L$ (Rao, 1992)

Alternatively, equation 2.33 can be expressed as:

$$
\begin{gathered}
R=P(L<S) \\
d R=P(L<S)=f_{S}(s) d s \int_{-\infty}^{S} f_{L}(l) d l=f_{S}(s) d s F_{L}(s)=A_{S}^{\prime} \cdot A^{\prime}{ }_{l} \\
R=\int d R=\int_{-\infty}^{\infty} f_{S}(s)\left[\int_{-\infty}^{S} f_{L}(l) d l\right] d s=\int_{-\infty}^{\infty} f_{S}(s) F_{L}(s) d s
\end{gathered}
$$

Figure 38 gives a visual explanation of equation 2.37 . 


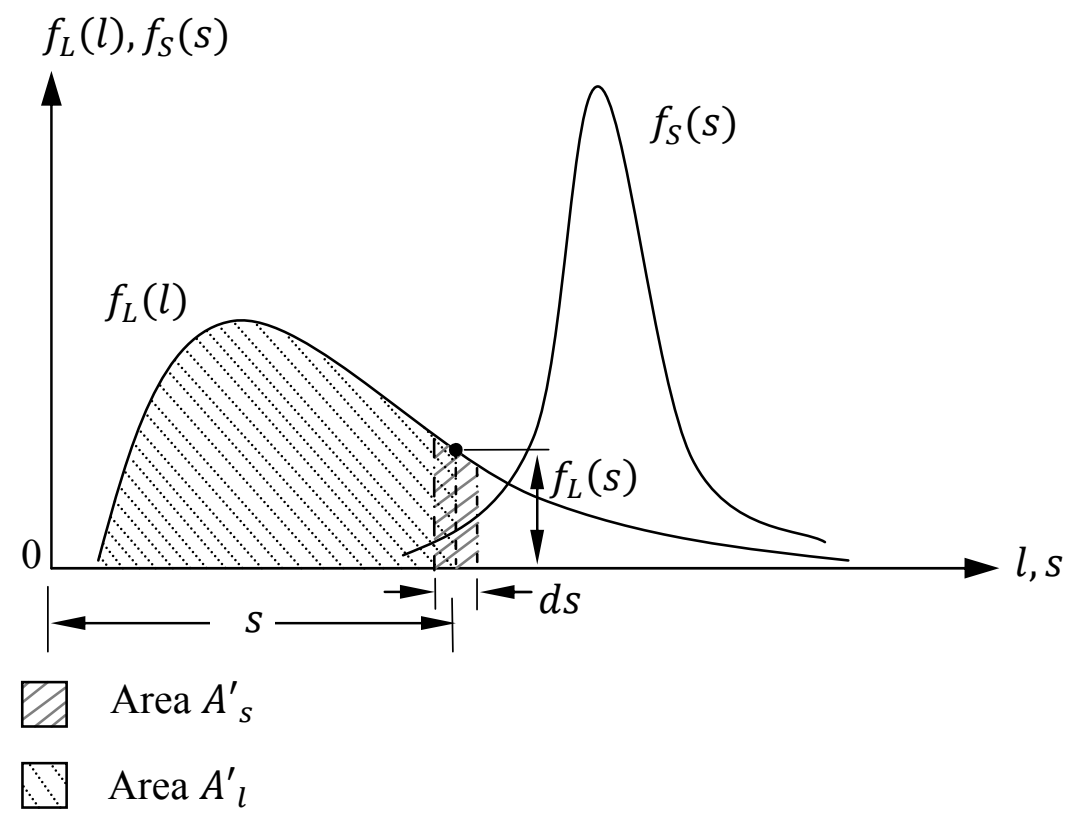

Figure 38: Definition of $d R$, for $L<S($ Rao, 1992)

The probability of failure, which is $1-R$, can be seen as the overlapping area between the load and the strength probability distributions, as shown in Figure 39.

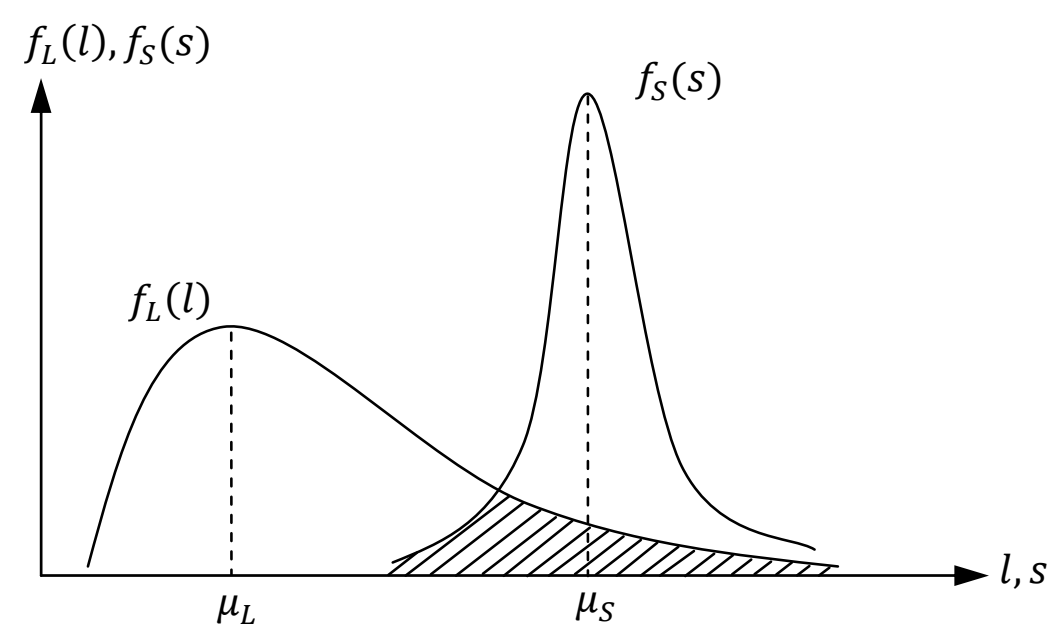

Figure 39: Interference Area, Probability of Failure (Rao, 1992) 


\subsection{OBJECTIVES OF THESIS}

The objectives of this thesis are listed below. The details will be discussed in later chapters.

- Perform and modify existing Non-Destructive Testing techniques: use Fibre Image Analysis to find fibre orientations and to quantify "fisheyes."

- Use optical microscope and built-in software, analySIS, to analyse dry CFRP textiles, textiles that have not been made into laminates, to find the fibre orientation (MFIA technique) and to quantify "fisheyes."

- Modify existing "prediction of failure" models: Fibre Micro-Buckling (FMB) Model, Ply Meso-buckling (PMB) Model

- Use modified "prediction of failure" models and Unit Cell Model with fibre orientation data, "fisheye" data, and fibre waviness data (to be discussed later) to calculate the compression strength.

- Compare compression testing results (Destructive Testing) with compression strength predictions.

- Develop a "reliability model" based on fibre orientation and compression testing results (Destructive Testing) to find the reliability, $R$, of a textile, as a measure of textile quality.

The next chapter, Chapter 3, discusses the experimental parameters specific to this thesis. Chapter 4 discusses the reliability model, and Chapter 5 discusses the results and conclusion. 


\section{CHAPTER 3: ANALYSIS OF MEASUREMENTS OF COMPOSITE DEFECT}

\section{OUTLINE OF CHAPTER 3}

- Input parameters for the FMB-PMB model will be thoroughly described: SFI and SFO. The ways the files and programs work will also be discussed.

- The way that data is acquired through scanned images, the software used, and how the study will progress through the analysis and computation of data will be discussed.

- The materials used will be thoroughly discussed: the arrangement of layers and material properties.

- Image analysis to find the SFI and "fisheyes" will be described.

- The way the SFO is found will also be discussed.

In this thesis, measurements were taken to quantify defects that were used to predict their influence on a failure due to compression. These predictions were compared to the compression testing results. A modified version of S. Pansart's FMB PMB Model was used to find the compression strength prediction. The result was combined with the Unit Cell Model to analyse the influence of "fisheyes" or "stitching/knitting yarn distortions" on the compression strength. This process was repeated by using software, Fibre Image Analysis, developed by the Faserinstitut Bremen (FIBRE) e.V. (Bremen Fibre Institute) at the University of Bremen in Germany.

\subsection{INPUT PARAMETERS}

In the adapted FMB-PMB Model, the input parameters used for the prediction of compression strength are: SFI, standard deviation of in-plane misorientations, and SFO, standard deviation of out-of-plane misorientations. These two values represent the two dimensional and three dimensional fibre deviations from being perfectly straight, the ideal case.

In addition to fibre deviations that could influence the compression strength, there are also holes in the multi-axial warp knitted fabric due to the needle puncturing through the fabric, in order to sew the fabric together. These holes are colloquially called "fisheyes" and more officially called "knitting yarn distortions" or "sewing yarn distortions." The Unit Cell Model 
that is used to predict the influence of fisheyes on the compression strength requires the length and width of the fisheye as well as the distance between successive needle punctures into the textile that is perpendicular to the fibre direction of the fibre ply (stitch distance), and the distance between new pieces of thread in fibre direction of the ply (stitch length). More about this model will be described in later Sections, 3.2 and 3.4.2. This Section will focus on Pansart's model.

Based on Pansart's model, optical microscopy on laminate section cuts was used to find the SFI. In the studies after Pansart, the waviness of a textile was determined by the SFO and the SFI. Multiple Field Image Analysis (MFIA), which uses analySIS software developed by Olympus is used to find the SFI. In this thesis, another method, using Fibre Image Analysis, is used to find the SFI. Both analySIS and Fibre Image Analysis is used in this thesis to analyse scanned images of textiles for SFI and "fisheyes." The next Sections, 3.2 to 3.5.1, will give more details on these measurement techniques.

The composite ultimate compression strength prediction was calculated according to the steps illustrated in Figure 40. Figure 40 not only illustrates the current, but also the previous way that the compression strength predictions were calculated. The dashed line represents the new way, the way that is used in this study. This new way is called "Estimation of Compression Strength on Basis of the SFO." The old ways is called "Estimation of Compression Strength on Basis of Critical Combination." More detail on these two methods will be described later.

As described in Section 2.3 in Chapter 2, according to Pansart, the purpose of the FMB model is illustrated by equation 2.23. The FMB Patran model is a two-dimensional model that models a piece of carbon fibre in resin under compression. In order to run this model, preprocess and post-process session files and an input file are required. The session files tell Patran what to do and the input file specify parameter values. The pre-process session file contains the instructions to create the model, such as the geometry, finite element mesh, boundary conditions, applied loads, material properties, element properties, and submitting the model to the solver. The solver is Nastran, which solves for displacements, computes strains, and computes stresses. With the results from the solver, the post-processing session file gives instructions on creating deformation plots, stress fringe plots, and reports. The resulting report file contains: $x$ displacement, $y$ displacement, force, and the maximum shear stress. However, the $y$ displacement and the maximum shear stress are not used in the FMB calculation. 


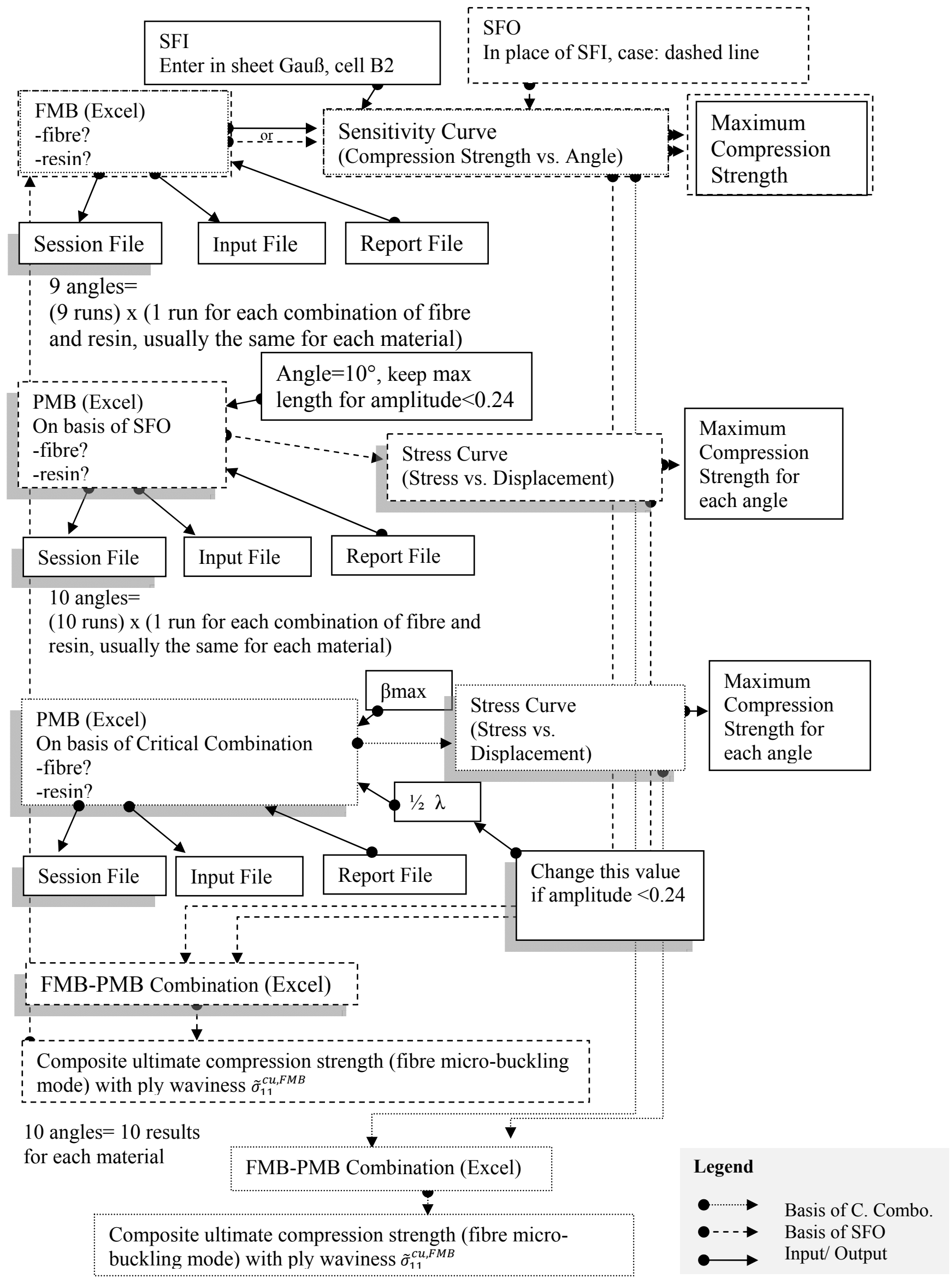

Figure 40: FMB and PMB Calculations for Prediction of Compression Strength 
As illustrated in Figure 40, an Excel file is used to automate the process in order to create the session files and the input file (top left). The resulting report file from Patran is also imported into this Excel file for further analysis.

It is worth mentioning that before the FMB model is run, an angle of initial deformation of the fibre needs to be specified. In this study, 9 angles, $\alpha$, were used: $0.1^{\circ}, 0.2^{\circ}, 0.4^{\circ}, 0.8^{\circ}, 1.6^{\circ}$, $3.2^{\circ}, 4.5^{\circ}, 5^{\circ}$, and $6^{\circ}$. For each angle, a new input file is created, thus a new report file is generated. Since the model geometry and other properties were the same for each angle, the session files were the same for each angle. The input file will change depending on the fibre and resin specified, and the angle of deformation of the fibre. With the report file imported into the FMB Excel file, macros in this file will calculate the failure stress from the force for each angle. The failure stress or failure force occurs when a sudden drop in stress or force is experienced. With all of the report file results imported into the FMB Excel file, a sensitivity curve, $\sigma_{\text {crit }}\left(\alpha_{0}\right)$, is created, which is a plot of the failure stress versus the angle.

Barbero (1998) established a Gaussian distribution for the best representation of the misalignment angle of each fibre in a composite laminate. The Gaussian probability density function is:

$$
f(\alpha)=\frac{1}{\Omega \sqrt{2 \pi}} \exp \left(\frac{-\alpha^{2}}{2 \Omega^{2}}\right), \quad-\infty<\alpha<\infty
$$

With this function and setting the misalignment angle, $\alpha$, to interpolated values of those 9 angles that were previously mentioned, $F\left(\alpha_{0}\right)$ is found for the interpolated angles. Here, $\Omega$ is the standard deviation of fibre in-plane (SFI) value. Also, interpolation is performed for the $\sigma_{\text {crit }}\left(\alpha_{0}\right)$ values. $F\left(\alpha_{0}\right)$ and $\sigma_{\text {crit }}\left(\alpha_{0}\right)$ are multiplied together to form a new curve. The maximum of this resulting curve is defined as $\sigma_{11}^{c u}$, the compression strength prediction due to fibre micro-buckling, FMB. $F\left(\alpha_{0}\right)$ and $\sigma_{\text {crit }}\left(\alpha_{0}\right)$ are then later used in the combined FMB PMB model.

As described in Section 3.3 in Chapter 3, according to Pansart, the purpose of the PMB model is illustrated by equation 2.25. The PMB Patran model is a two-dimensional model that models a stack of plies of carbon fibre textile in resin under compression. Like the FMB model, the Patran model is run with the pre-process session file, post-process session file, and an input 
file. Like for the FMB model, the session files will be the same in any given situation. The input file will change depending on the fibre and resin specified and the amplitude of deformation of the middle $0^{\circ}$ ply; the amplitude will be discussed later. The resulting report file contains the $x$ displacement, $z$ displacement, force, and stresses in the $x, z$, and $x z$ direction for each of the 10 nodes $(5.1$ to $5.5,6.1$ to 6.5$)$ inside the middle $0^{\circ}$ ply in the model and for the interleave, as shown in Figure 29. Just like the FMB model, an Excel file is used to automate the process of creating the session files and the input file, as illustrated in Figure 40 (middle). The resulting report file from Patran is also imported into this Excel file for further analysis.

Just like the FMB model, an angle of deformation of the middle $0^{\circ}$ ply needs to be specified for PMB on basis of SFO, the method used in this study. Ten angles, $\beta$, were used: $0.1^{\circ}, 0.2^{\circ}, 0.4^{\circ}, 0.8^{\circ}, 1.6^{\circ}, 3.2^{\circ}, 4.5^{\circ}, 5^{\circ}, 6^{\circ}$, and $10^{\circ}$. For each of these angles, the total length of the model is kept constant, at a value that for the amplitude is less than 0.240 for an angle of $10^{\circ}$, recall equation 2.20. This is done to prevent the layers in the model from overlapping, due to the "wave" in the middle $0^{\circ}$ ply. For each angle, a new input file is created, thus a new report file is generated. Since the model geometry and other properties were the same for each angle, the session files were the same for each angle. With the report file imported into the PMB Excel file, macros in this file will make graphs from the report file data, stress versus $x$ displacement. The failure stress or failure force occurs when a sudden drop in stress or force is experienced. Immediately after importing each report file into the PMB Excel file, macros in the Excel file will take the contents of the last imported report file and sort it. The sorted data from the report file is then saved in a separate Excel, to be used in the combined FMB PMB model. The report file in the PMB model contains the stresses, $\sigma_{11}, \sigma_{33}, \tau_{13}$, in each of the 10 nodes that were previously discussed. Stress in the in-fibre direction, $\sigma_{11}$, represents the sum of the stresses in the 10 nodes for a corresponding value of $x$ and $z$ displacement and the force in the in-fibre direction. Likewise, $\sigma_{33}$ and $\tau_{13}$ represent the sum of the stresses in the 10 nodes for a corresponding value of $x$ and $z$ displacement and force in the transverse direction and in the shear direction.

The PMB model on basis of critical combination does not require the angles, $\beta$, to vary. So, a sensitivity curve for a continuum damage model is not created. This was the original model that Pansart used. It was assumed in Pansart's model that the entire laminate will immediately fail after one $0^{\circ}$ ply has failed, instead of a slow failure that was a result of the $0^{\circ}$ ply buckling and the remainder of the plies carrying the load. In the case of the FMB model, it is assumed that 
there is a slow failure that is a result of a fibre buckling and the remainder of the fibres carrying the load.

The PMB model on basis of critical combination functions in a similar way that was described for the PMB model on basis of SFO. Instead of varying the angles $\beta$, the combination of the half wavelength, total length of the model, and the maximum gradient, maximum $\beta$, is used for the amplitude to be less than 0.24. The half wavelength and the maximum gradient are found from waviness measurements, which will be described later.

The FMB PMB combination is done completely using Excel. The results from the FMB and PMB model is combined to find a compression strength prediction based on the fibres that are not straight and based on plies that are wavy. $F\left(\alpha_{0}\right)$ and $\sigma_{\text {crit }}\left(\alpha_{0}\right)$ are taken from the FMB model. The sorted data, which corresponds to $\sigma_{11}, \sigma_{33}, \tau_{13}$, from the PMB report file are taken from the PMB model. Using $\sigma_{11}, \sigma_{33}, \tau_{13}$, equation 2.27 is used to find $\varphi$. Then, equation 2.28 is used to find $\alpha_{e q}$, the equivalent initial fibre angle, due to the fibre angle misalignment and ply waviness. To calculate $\sigma_{\text {crit }}\left(\alpha_{\text {eq }}\right)$ using linear interpolation, a value that varies based on $\alpha_{e q}$, interpolating between values $\sigma_{\text {crit }}\left(\alpha_{0}\right)$ and $\alpha_{0}$ is used. Then, allowing $\sigma_{\text {crit }}\left(\alpha_{\text {eq }}\right)$ to be the new sensitivity curve, thus $\sigma_{\text {crit }}\left(\alpha_{\text {eq }}\right)=\sigma_{\text {crit }}\left(\alpha_{0}\right)$, the ultimate compression strength $\tilde{\sigma}_{11}^{c u, F M B}$ from equation 2.23 is found through $\max _{\alpha_{0}}\left[\sigma_{\text {crit }}\left(\alpha_{\mathrm{eq}}\right) \cdot F\left(\alpha_{0}\right)\right]$. This process is repeated for each new combination of $\varphi$ based on displacement and the nodes (5.1 to 5.5, 6.1 to 6.5). This would result in a matrix of ultimate compression strength values. Correspondingly, there will a matrix of local principal compression strength values, $\sigma_{11, e q}$, from equation 2.26. Based on equation 2.30 , at the first value that $\sigma_{11, e q}$ divided by $\tilde{\sigma}_{11}^{c u, F M B}$ is equal to or greater than 1 , there is a failure. This is the compression strength prediction for the UD ply. This is the final value used in FMB PMB on basis of critical combination.

If the FMB PMB on basis of SFO is used, the compression strength prediction, $\tilde{\sigma}_{11}^{c u, F M B}$, for the UD ply is found for each varying angle, $\beta$. Then, each value is used to create a sensitivity curve that is placed in the FMB Excel file. This is done because the steps are exactly the same as that for the FMB analysis. In the FMB Excel file, the sample standard deviation of fibres out-ofplane (SFO) is used instead of the sample standard deviation of fibres in-plane (SFI). The difference in how these values are obtained will be described next. 


\subsection{DATA ACQUISITION}

Three types of data have been acquired for this study: Standard deviation In-plane (SFI), Standard deviation Out-of-Plane (SFO), and "fisheye" or stitching distortion data such as: width, length, as well as stitch distance and stitch length, which are within and between thread distances of "fisheyes," respectively. These three types of data are further used to determine the compression strength of a CFRP laminate, which will be further described in the next Sections, 3.4.1 to 3.5.1.

For this study, the standard deviation in-plane (SFI) value was found through sampling carbon fibre textiles, with microscope images. According to the MFIA technique, "the old technique," using analySIS software, a sample of textile was cut into two pieces, two trials. Having two trials was performed to increase the number of measurements, in order to find results that better represented the textile. The two samples were used to find two separate SFI values. The Excel macros were only able to handle results from one sample. Therefore, the macros were revised to combine the SFI values of the two samples to create one SFI value. Also, for each sample of textile, there are two sides, and this technique is performed on each side of the textile. As Figure 41 illustrates, the SFI value feeds into the FMB model. The result of the FMB model is the failure stress of each angle $\left(0.1^{\circ}, 0.2^{\circ}, 0.4^{\circ}, 0.8^{\circ}, 1.6^{\circ}, 3.2^{\circ}, 4.5^{\circ}, 5^{\circ}, 6^{\circ}\right.$, and sometimes $10^{\circ}$ ) of the fibre, as found with Patran.

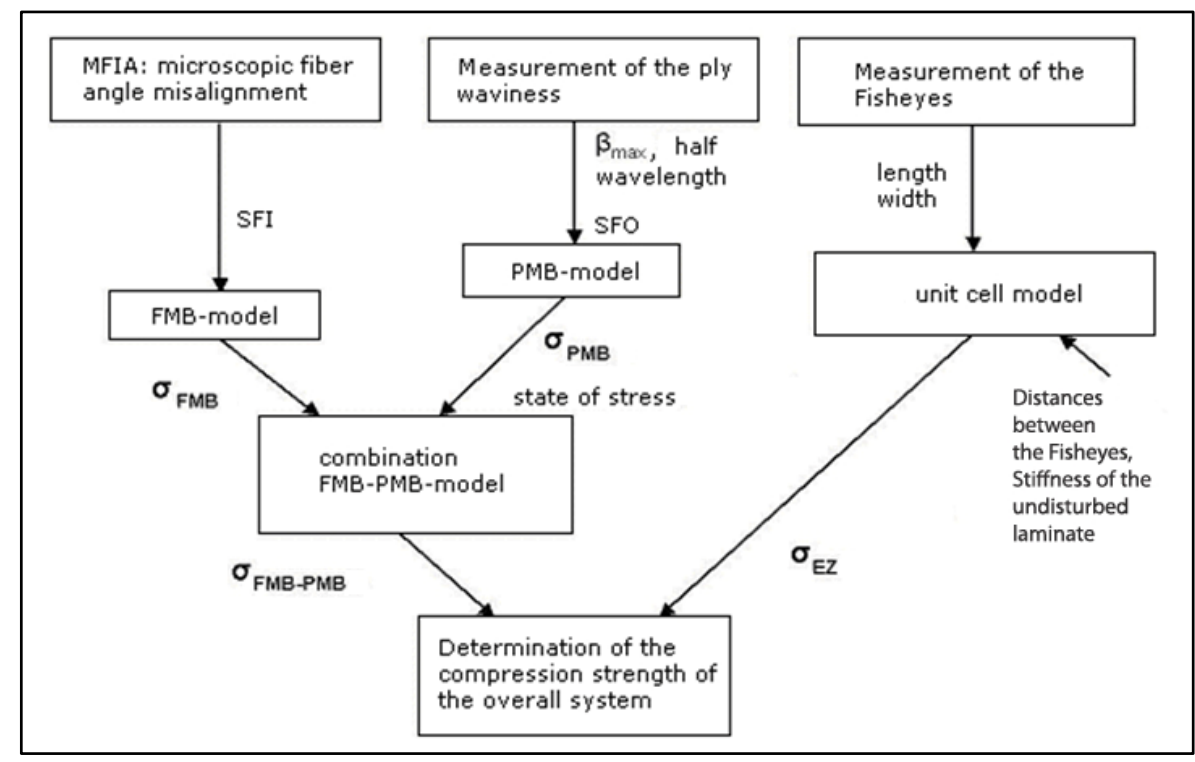

Figure 41: Determination of Compression Strength of Overall System (Rehbein, 2009) 
The results of the MFIA technique were compared to a newer technique that uses software by the the Faserinstitut Bremen (FIBRE) e.V. (Bremen Fibre Institute), Fibre Image Analysis. This software is capable of taking images, images created by scanning a textile sample on a flatbed scanner, and analysing them for the orientation of the fibres and the length and width of the "fisheyes." However, Fibre Image Analysis was specifically created for analysing images of photographed textiles in tension. For comparison with the results from analySIS, $2400 \mathrm{dpi}$ scanned images of the textiles were used with Fibre Image Analysis, as shown in Table 2.

\begin{tabular}{|l|l|l|}
\hline $2400 \mathrm{dpi}$ & analySIS & Fibre Image Analysis \\
\hline $720 \mathrm{dpi}$ & Fisheye & Fibre Angle (SFI) \\
\hline Microscope & Fibre Angle with MFIA (SFI) & $\begin{array}{l}\text { Fibre Angle (SFI) } \\
\text { (only for one image, for } \\
\text { comparison purposes only) }\end{array}$ \\
\hline
\end{tabular}

Table 2: SFI and Fisheye, Comparison of Techniques

Also analysed for SFI are 720 dpi images, to be used as a comparison with 2400 dpi images. This was done to deal with the event that something goes wrong with the 2400 dpi images. In addition, one microscope image was analysed with Fibre Image Analysis. This was done to see if there is a substantial difference due to the Fibre Image Analysis software, and not the method for capturing the image.

The standard deviation out-of-plane (SFO) is also found through sampling carbon fibre textiles, by using a device with a pin that moves across the surface of the textile which measures the waviness of that surface. There are two samples of a textile, an effort to try to see if there are any major differences between two samples of a textile. For each sample of a textile, there are five waviness measurements for each side of the textile. For 0/90 textiles, described more thoroughly in the next Section, 3.3, one side is labelled the "up side" and one the "down side." These names were given to the way the roll of textile is often unrolled onto the table for cutting. The "up side" is also called the $0^{\circ}$ direction and the "down side" the $90^{\circ}$ direction. For the $0^{\circ}$ 
direction, the general direction of the fibres is in the same direction of the sewing thread that holds the textile together, whereas for the $90^{\circ}$ direction, the general direction of the fibres is perpendicular to the sewing thread. Further, waviness is measured in two directions: one along the general direction of the fibres and one perpendicular to the fibres for each side of the textile. The results of only one of these two directions are actually needed, depending on which side of the textile the waviness is being measured. For the "up side," $0^{\circ}$ direction, the waviness results along the direction of the fibres should be used, and vice versa for the "down side." Waviness results from both directions are taken only for cross checking purposes. In addition to the SFO value that results from the waviness measurements, the $\beta_{\max }$, which is the maximum gradient, and the half wavelength are also determined. These two values are used in the PMB model on basis of critical combination, which was not used in this study but in previous studies. They correspond to the maximum angle of deflection of the pin that moves across the surface of the textile and the distance moved by the pin for this deflection angle. As shown in Figure 41, the SFO value feeds into the PMB model. The result is the force from Patran that causes the middle $0^{\circ}$ ply to fail for each angle of inclination of the middle $0^{\circ}$ ply $\left(0.1^{\circ}, 0.2^{\circ}, 0.4^{\circ}, 0.8^{\circ}, 1.6^{\circ}, 3.2^{\circ}\right.$, $4.5^{\circ}, 5^{\circ}, 6^{\circ}$, and sometimes $10^{\circ}$ ).

Next, the FMB and PMB results are combined using Excel macros. On basis of SFO, as long as there is a failure stress value for FMB and a failure force value for PMB, there will be a combined result for each angle of inclination $\left(0.1^{\circ}, 0.2^{\circ}, 0.4^{\circ}, 0.8^{\circ}, 1.6^{\circ}, 3.2^{\circ}, 4.5^{\circ}, 5^{\circ}, 6^{\circ}\right.$, and sometimes $10^{\circ}$ ). As described in Section 3.1 in Chapter 3, the ultimate compression strength, $\tilde{\sigma}_{11}^{C u, F M B}$, is found for each angle. The ultimate compression strength could occur at any of the nodes described earlier (5.1 to $5.5,6.1$ to 6.5$)$. Then, the ultimate compression strength associated with an angle is combined to find the compression strength prediction. As previously described, this is performed by creating a sensitivity curve through the macros contained in the FMB Excel file and using the SFO value instead of the SFI value.

The "fisheyes" or stitching yarn distortions, holes caused by the stitching of the thread through the carbon fibres to form a fabric, can also have an impact on the quality of the laminate. These holes are characterised by their length and their width, as well as the distance between the holes. More about this model will be described later, in the Section dedicated to "fisheyes," 3.4.2. Based on these parameters, the Unit Cell model, a model that was funded by Institut für Verbundwerkstoffe (IVW) (Institute of Composite Materials) based at the TU Kaiserslautern 
(University of Kaiserslautern), is a FE-Model used to simulate the influence of stitching: the length, width, stitch distance, and stitch length of "fisheyes." In the Diploma thesis of Lam Pham (Lam, 2008), a database was created that allows the compression strength values to be found by cubic interpolation between known values.

In order to find the total effect of the "fisheyes," the waviness of the textile, and the distortion of the fibres in a textile, a knock-down factor needs to be applied to the FMB PMB combined result. This knock-down factor is based on how much of an impact the "fisheyes" have on an undisturbed laminate. An undisturbed laminate is a perfect laminate that does not have any quality issues resulting from stitching. The knock-down factor is the compression strength of an undisturbed laminate divided by the compression strength of the disturbed laminate. The resulting number should be between 0 and 1 . This number is then multiplied to the compression strength prediction found from the FMB PMB combined model. The final result is the compression strength of the overall system.

\subsection{MATERIALS AND MATERIAL PROPERTIES}

A total of 8 different carbon fibre textiles were analysed in this study. Out of these 8 textiles, 6 are considered to be 0/90 textiles. The other two textiles are 135/0/45/90 and 45/0/135 which are together used to form one laminate. The laminates are infused with epoxy resin.

For the 0/90 textiles, two layers of carbon fibres are stacked at $90^{\circ}$ from each other. These two layers are sewn together to form the textile. One layer, the one with the stitching thread in the direction of the fibres, is called the $0^{\circ}$ layer. Likewise, the layer with the stitching thread perpendicular to the fibres is called the $90^{\circ}$ layer.

As shown in Figure 42, 0/90 laminates used in this thesis are composed of 8 layers of 0/90 textiles. Here, it is shown that top 4 layers are stacked in the 0/90 arrangement, where the $0^{\circ}$ layer is on top. Also, the bottom 4 layers are stacked in the 90/0 arrangement, where the $90^{\circ}$ layer is on the bottom. The Modified Vacuum Infusion technique, similar to the Vacuum Infusion technique described in Section 1.1 in Chapter 1, is used to infiltrate the cut textiles to form the laminates. The resulting laminate is about $4 \mathrm{~mm}$ thick. For most of the 0/90 textiles, the areal weight of the textile, usually $267 \mathrm{~g} / \mathrm{m}^{2}$, is the same for the $0^{\circ}$ layer and the $90^{\circ}$ layer, thus the resulting $0 / 90$ laminate would have proportion of $0^{\circ}$ to $90^{\circ}$ to be $50 \%$ to $50 \%$ or $50 / 50$. There are two textiles that have an areal weight of $282 \mathrm{~g} / \mathrm{m}^{2}$ for the $0^{\circ}$ layer and $268 \mathrm{~g} / \mathrm{m}^{2}$ for the 
$90^{\circ}$ layer. The fibre density for the $0 / 90$ laminate is $1.78 \mathrm{~g} / \mathrm{m}^{3}$ and the fibre volume content is about $60 \%$. The six $0 / 90$ textiles differ from each other in terms of the way they are stitched together. For example, the stitching may be closer or farther apart and the tension tighter for some.

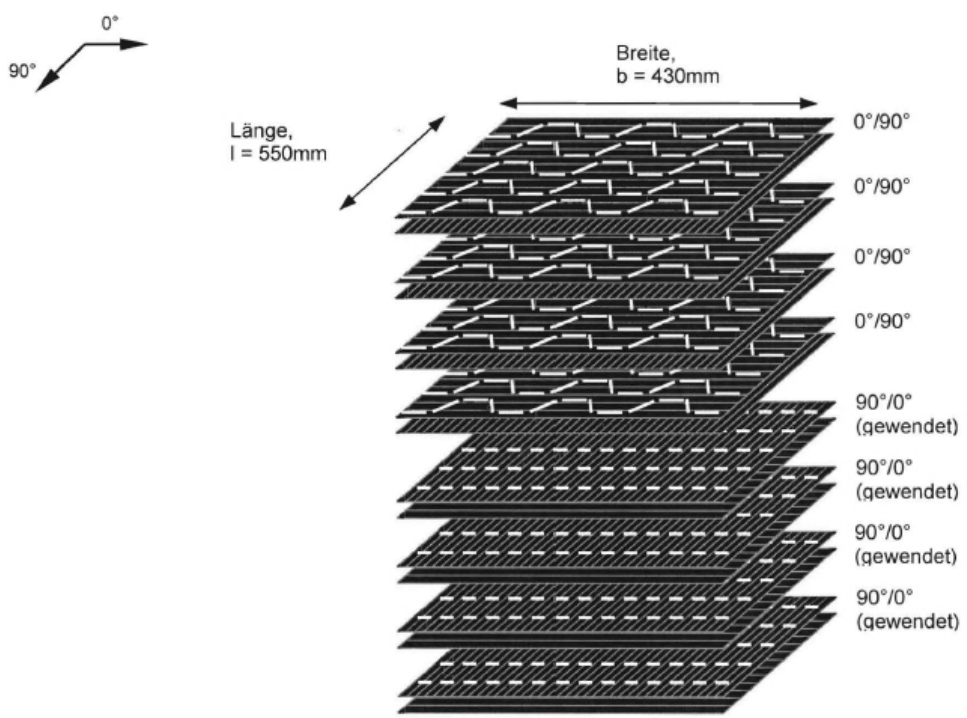

Figure 42: 0/90 Laminate Stacking

For the $135 / 0 / 45 / 90$ and $45 / 0 / 135$ textiles, they together form a laminate that is $50 / 40 / 10$. This means that $50 \%$ of the laminate can be contributed to the $0^{\circ}$ layer, $20 \%$ to the $45^{\circ}$ layer, $20 \%$ to the $135^{\circ}$ layer, and $10 \%$ to the $90^{\circ}$ layer. In the $50 / 40 / 10$ laminate, the areal weights are different for the layers. The $0^{\circ}$ layer has an areal weight of $394 \mathrm{~g} / \mathrm{m}^{2}$ and the other three layers have an areal weight of $160 \mathrm{~g} / \mathrm{m}^{2}$. The fibre density for the $50 / 40 / 10$ laminate is $1.8 \mathrm{~g} / \mathrm{m}^{3}$ and the fibre volume content is about $60 \%$.

An equation can be used to calculate the fibre volume content:

$$
\epsilon=\frac{\left(m_{l}-m_{a}\right) \cdot n}{\rho \cdot t \cdot 1000} \cdot 100 \%
$$

Where

$\epsilon$ is the fibre volume content [\%]

$m_{l}$ is the areal weight of the layer $\left[\mathrm{g} / \mathrm{m}^{2}\right]$

$m_{a}$ is the areal weight of everything except the carbon fibre $\left[\mathrm{g} / \mathrm{m}^{2}\right]$

$\rho$ is the fibre density $\left[\mathrm{g} / \mathrm{cm}^{3}\right]$ 
$t$ is the mean measured thickness of the laminate [mm]

$\mathrm{n}$ is the number of layers with the same areal weight of the layer

For four of the six 0/90 laminates, sheared laminates were produced. These laminates were made with textiles that have been sheared $15^{\circ}$ from the $0^{\circ}$ direction. Recall that a textile can have 2 samples, sample 1 and sample 2 . Shearing of the textile is only performed on sample 1.
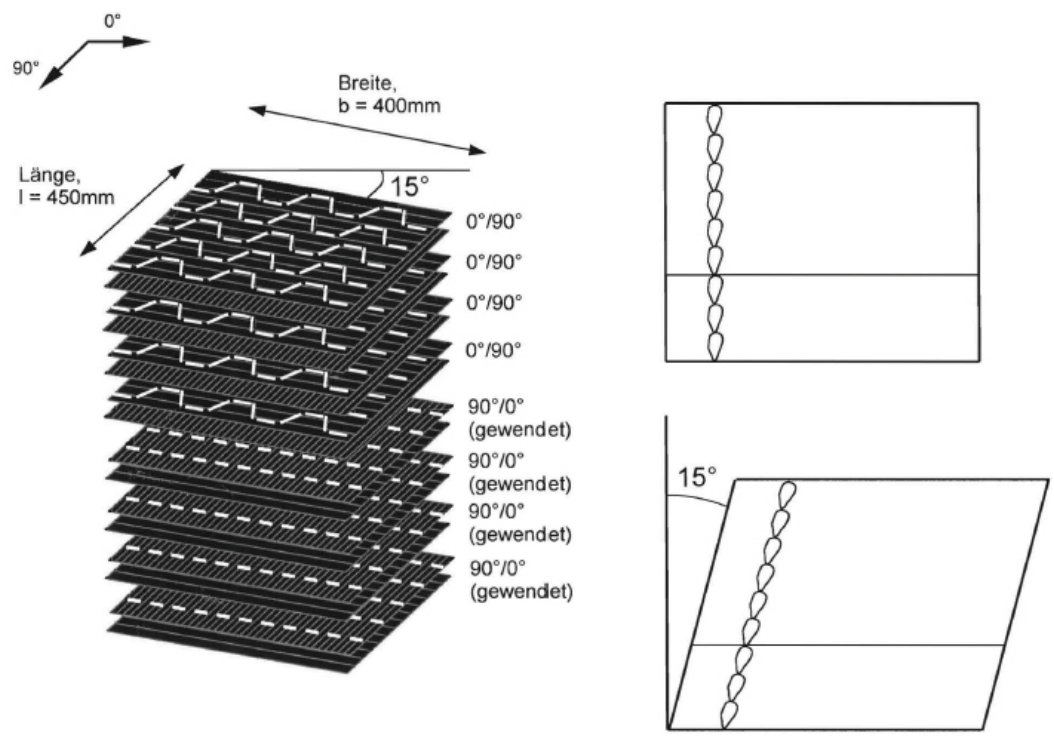

Figure 43: Sheared 0/90 Laminate Stacking

In the results, a textile can be classified into sample 1 or sample 2. For each sample, it can be further classified as up side or down side. The "side" classification identifies the side which the SFI, SFO, and fisheyes were found as well as how the orientation for the coupon for compression testing was cut. Loading for compression testing is always parallel to the fibre direction with respect to "side" classification.

\subsection{IMAGE ANALYSIS}

In order to find the sample standard deviation of fibres in-plane (SFI), and the length and width of "fisheyes," images of the carbon fibre textiles are analysed. These images can come in the form of microscope images, as for the MFIA method for finding the SFI, or in the form of flatbed scanner images. Software such as analySIS can be used to analyse these images as well as the Fibre Image Analysis. 


\subsubsection{Sample Standard deviation of Fibre In-plane (SFI)}

\subsubsection{SFI with MFIA}

The SFI value refers to the sample standard deviation of fibres in-plane. Traditionally, the method used to find this value is through optical microscopy and the Multiple Field Image Analysis (MFIA) technique, as described in Section 2.2.2.1 in Chapter 2. A similar method to the MFIA technique used by Creighton et al. (2001) was used in this study. The MFIA technique involves cutting a sample of textile, placing it in a clamping device, using a microscope, Olympus SZX12, with an attached camera, CC12 Soft Imaging System, for a digital output to a computer monitor. When the desired image is acquired through zooming and focusing, the image is then captured. Then, further analysis is performed on the image using analySIS software. The analySIS software is packaged with the Olympus microscope. The software company that owns analySIS is Soft Imaging Solution, Five Digital Imaging Solutions, based on analysis platform. With code that is specifically written to analyse the image based on light and dark areas, the average fibre angle for each domain is determined. By default, there are 54 domains in each image. In the rectangular "overview" window seen in Figure 44 (b), 7 magnified microscope images were taken in each trial. As mentioned in Section 3.2 in Chapter 3, two samples were cut for each textile, and two additional samples were cut from each sample. These two additional samples are called "trials" for the purpose of nomenclature in this study. These 7 magnified microscope images, captured at 90x, were taken in an area where there was no sewing thread or other abnormalities, such as tangled strands of carbon fibre threads as shown in Figure 45. This was required to prevent the sewing thread and other abnormalities from affecting the SFI result, which is supposed to be strictly about the orientation of the carbon fibres.

As depicted in Figure 44, the trial is cut in such a way that the fibres are oriented in a $45^{\circ}$ angle with respect to the square shape of the cut. The trial is about $8 \times 8 \mathrm{~cm}$, with space in the clamping device for the trial to move around. This is beneficial to better represent the textile because it is better to have a larger sampling area of the textile. It is better to capture the 7 magnified microscope images from all around the surface of the trial instead of within the small "overview" window. Also, the trial has the edges taped to prevent frilling or ruffling of the edges. 

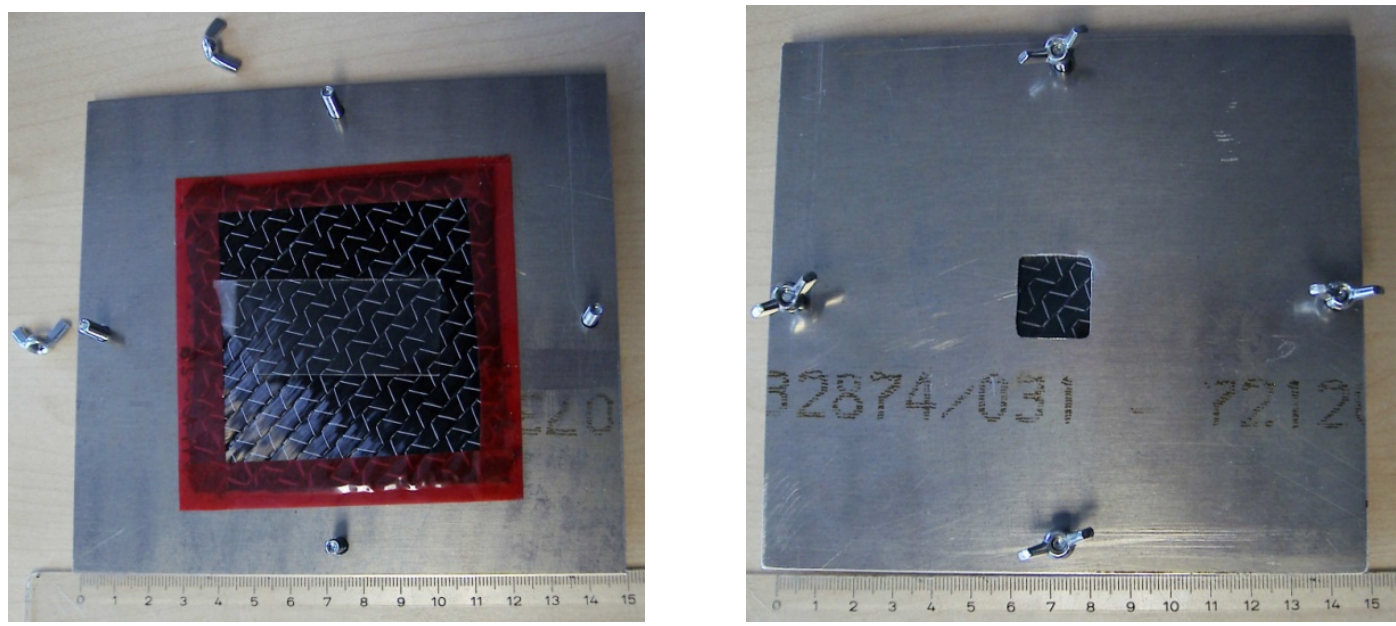

Figure 44: "Trial" in MFIA clamping device, a) without lid b) with lid

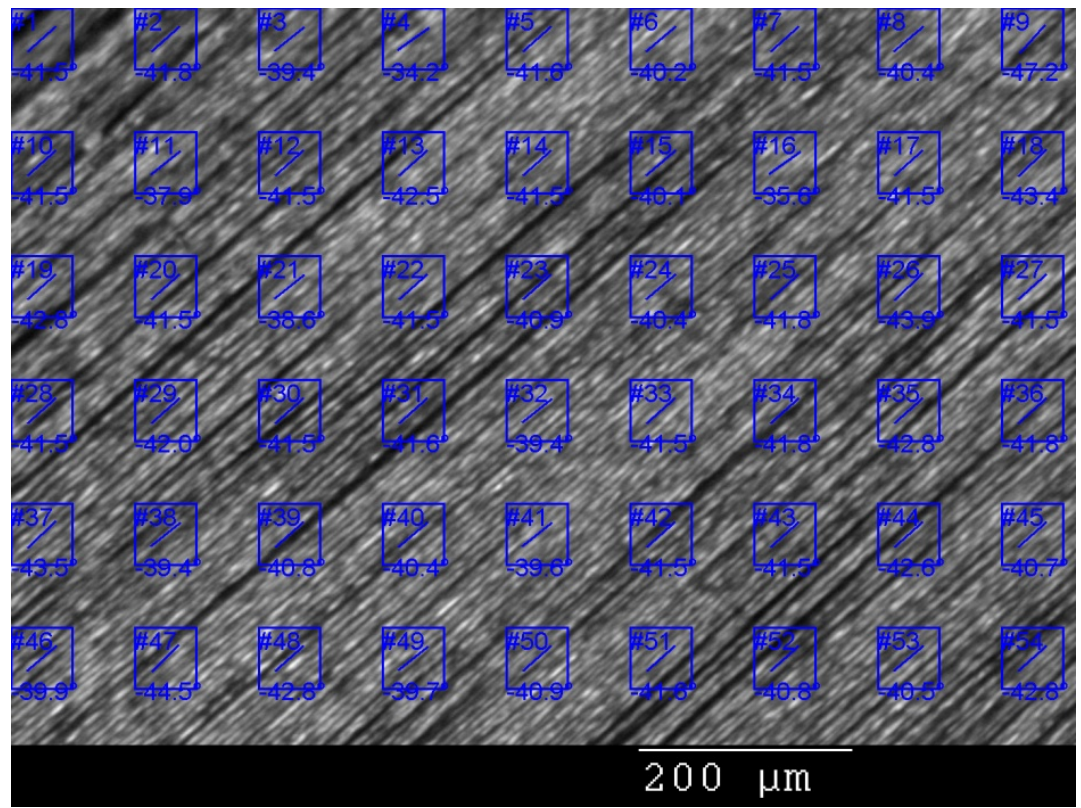

Figure 45: MFIA Microscope Image with Domains

From Figure 45, it can be derived how the 54 domains are sampled across the captured microscope image. Table 3 and Figure 46 summarises how the domains were created and how the average fibre angle was found. Each domain is numbered and contains an arrow that represents the average fibre angle found for that domain. A domain represents an area where the fibre angle was sampled. Inside a domain, a maximum of $50 \mathrm{~N}$ points were taken as reference points. Then, an array of 40 pixels, including the middle anchor point that did not turn, was created from these $N$ points to determine the average fibre angle. In order to determine the average fibre angle from the array, a range for the angles to be tested needs to be specified. Also, 
a narrow range of $10^{\circ}$ has been specified to make the program to run faster. At an angle step sizes of $0.1^{\circ}$, the program will test the array of pixels from the start angle of $-50^{\circ}$ to $-40^{\circ}$ for the angle that best describes the fibre orientation of the point chosen. Recall that the trials have been cut so that the fibres were oriented in $45^{\circ}$. It has been concluded from experience that this angle gives the most meaningful results with analySIS and avoids the confusion of having negative result values. Once a run has been completed, an Excel file will be generated, which lists the average fibre angle for each of the 54 domains. Seven microscope images were taken over the surface of a trial. There were 2 trials.

\begin{tabular}{|c|c|c|}
\hline & Pixel & $\mu \mathrm{m}$ \\
\hline Domain width & 80 & 58 \\
\hline Domain height & 80 & 58 \\
\hline Distance between domain in $x$ direction & 80 & 58 \\
\hline Distance between domain in $y$ direction & 80 & 58 \\
\hline Length of displayed arrow & 50 & 36 \\
\hline Length of array & 40 & 29 \\
\hline Maximum number of $N$ points & 50 & \\
\hline Start angle & -50 & \\
\hline Angle length & 10 & \\
\hline Angle step interval & 0.1 & \\
\hline
\end{tabular}

Table 3: MFIA Domains

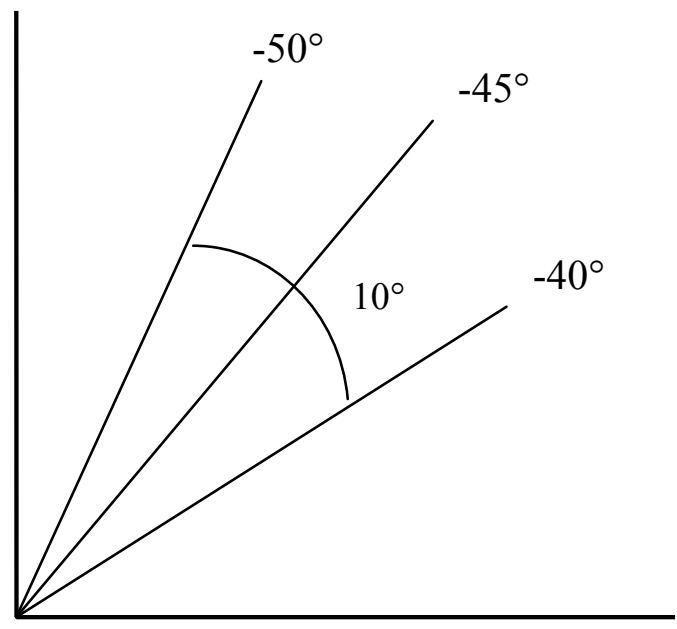

Figure 46: MFIA Angle Interval 
Later, an Excel macro calculates the SFI from the results of each domain in each image. This is done by finding the average fibre angle orientation of the entire image by averaging the fibre angle value for each domain. The difference between the average fibre angle orientation of the entire image and the average fibre angle orientation in each domain of the image was calculated for each domain. In the past, only 7 images from 1 sample were taken. With this arrangement, the SFI of the sample was calculated as the standard deviation across all of this difference across all of the images. Here, a large sample was further cut into two smaller pieces, two "trials," with 7 images per trial, and a total of 14 images per sample. This is a modification from the original setup. An equation was derived, by the author, for this study, to find the representative SFI value for these two trials. This equation assumes that there was independent sampling. This means that the selected areas of the textile used to find the SFI value have no effect on one another. The sample variance is given by:

$$
\sigma_{t}^{2}=\frac{\sigma_{a}^{2}\left(N_{a}-1\right)+\sigma_{b}^{2}\left(N_{b}-1\right)+N_{a} \mu_{a}^{2}+N_{b} \mu_{b}^{2}-N_{t} \mu_{t}^{2}}{N_{t}-1}
$$

Where,

$N_{a}$ is the number of items in trial A

$N_{b}$ is the number of items in trial B

$N_{t}=N_{a}+N_{b}$

$\Sigma \mathrm{a}$ is the sum of items in trial A

$\Sigma \mathrm{b}$ is the sum of items in trial B

$\sum t=\sum a+\sum b$

$\Sigma \mathrm{a}^{2}$ is the sum of squares of items in trial A

$\Sigma \mathrm{b}^{2}$ is the sum of squares of items in trial B

$\sum t^{2}=\sum a^{2}+\sum b^{2}$

$\mu_{\mathrm{a}}$ is the sample mean of trial A

$\mu_{\mathrm{b}}$ is the sample mean of trial B

$\mu_{\mathrm{t}}$ is the sample mean of the combined trials

$\sigma_{\mathrm{a}}$ is the sample standard deviation of trial A

$\sigma_{b}$ is the sample standard deviation of trial B

$\sigma_{\mathrm{t}}$ is the sample standard deviation of the combined trials 
This equation is equivalent to finding the sample standard deviation of the differences between the average fibre orientation of an image and the domain values for the 14 images. The advantage of the equation is that it simplifies the steps to calculate the standard deviation. This is especially true if there are more than 2 trials. Referring to the steps outlined in Appendix A, equation 3.3 can be modified to include more trials. Equation 3.3 can be modified to find the SFI for 4 trials as follows:

$$
\sigma_{t}^{2}=\frac{\sigma_{a}^{2}\left(N_{a}-1\right)+\sigma_{b}^{2}\left(N_{b}-1\right)+\sigma_{c}^{2}\left(N_{c}-1\right)+\sigma_{d}^{2}\left(N_{d}-1\right)+N_{a} \mu_{a}^{2}+N_{b} \mu_{b}^{2}+N_{c} \mu_{c}^{2}+N_{d} \mu_{d}^{2}-N_{t} \mu_{t}^{2}}{N_{t}-1}
$$

\subsubsection{SFI with Fibre Image Analysis}

As mentioned earlier, another way was used to find the SFI value. This was through using Fibre Image Analysis software developed by the Faserinstitut Bremen (FIBRE) e.V. (Bremen Fibre Institute) at the University of Bremen in Germany. In Section 2.2.1.1 in Chapter 2, Miene et al. (2008) used software called "PROSA Faserwinkelsoftware." It is the predecessor of Fibre Image Analysis. Fibre Image Analysis is based on image processing algorithms from Intel ${ }^{\circledR}$ Computer Vision Library (OpenCV), and was developed and programmed only by Dr. Miene, with the requirements set out by Mr. Kuntz (same author as on the topic of "SFO," see reference list).

This method is a non-destructive way to find the SFI, as is the MFIA technique mentioned earlier. In this process, pieces of textiles are scanned using a flatbed scanner, instead of using microscope images as in the aforementioned MFIA technique, which is a process similar to the process by Schneider et al. (2004). As mentioned in Section 3.2 in Chapter 3, Fibre Image Analysis has been used to find the SFI value for both 720 and 2400 dpi images. It was also used to find the SFI value for a select number of microscope images.

Given a number of parameters that can be changed, the Fibre Image Analysis software creates a number of result files. These are different versions of the original scanned image, analysed under different conditions.

Among the parameters, there are only a few that play an important role in the final result, as concluded from the experiences of the author. In the case with scanned images, the parameters 
that will need to be changed are: background segmentation method, gap global threshold, and pixel per millimetre. The parameter that is recommended to be adjusted is mask threshold.

The background segmentation method parameter can have four values: $0,1,2$ or 3 . Having scanned images, there is no background behind the textile and no ruler. This is because they would have been removed using photo finishing software such as Corel Paint Shop Pro or the scan area has been carefully specified so that the background is not captured during the scanning process. Therefore, this parameter is assigned with a value of 0 (deactivated). Since some textile images may contain a background and a ruler, a number in the range of 1 to 3 is used for images with a background, with a ruler, or with both a background and a ruler, respectively.

The gap global threshold parameter is usually assigned a value of 0 (off). However, for scanned images, there is an undesirable possibility that many small adjacent holes are identified. These holes are classified as gaps ("fisheyes"), and this is due to a small neighbourhood size (mask window size) value. Therefore, the gap global threshold parameter is assigned a value of 10 , a value found from past experience.

Mask window size, as with mask threshold, is a parameter used to differentiate between the fibre and the sewing thread (knitting yarn, seam). A mask is used to remove the categorised sewing thread areas from all calculations, including finding the SFI value. Here, mask window size is an odd number, which specifies the square area that will be analysed. For example, if the mask window size value is five, then the area is a $5 \times 5$ pixel area. The centre of this square area is the pixel that is being analysed and categorised as fibre or sewing thread. The specified mask threshold value is subtracted from the average brightness of each pixel in the mask window size area, the $5 \times 5$ area. If the value (brightness) of the centre pixel is greater than the resulting value, then the centre pixel is classified sewing thread. Through shifting this window throughout the entire area of the scanned image, the pixels of the entire image are classified as fibre or sewing thread. As a result, if the mask threshold value is changed from -80 to -40 , more pixels will be classified as sewing thread. Care needs to be given to selecting the window size. It should be selected with respect to the image resolution. If the window is too small, only the outline of the sewing thread will be classified as sewing thread. From experience, it has been concluded that a mask threshold value of -80 , instead of the default value of -40 , works well with scanned images. 
The pixel per millimetre parameter is important since this specifies, to the software, how many pixels there are for 1 millimetre. This is necessary for the analysed results to be logical. This value would change depending on the resolution of the image. The resolution of the images is in "dpi," which stands for dots per inch, which is equivalent to pixel per inch. An image with a resolution in "dpi" can be converted to pixel per millimetre by dividing it by 25.4. This step is only performed for scanner images (720 dpi and $2400 \mathrm{dpi}$ ) and microscope images (200 dpi).

In the case of finding the SFI value, the parameters that need to be adjusted according to each image are: domain size, activate analysis function, save results, maximum standard deviation inside the domains, minimum edge percent, angle tolerance 1, angle tolerance 2, minimum percent unmasked, and maximum width seam millimetre.

The domain size parameter specifies the size, in pixels, of each sub-image in the entire image. The fibre angle for each sub-image will be later found and used to calculate the deviations of the fibre angles (SFI). The smallest domain size that can be specified is $50 \times 50$ pixels. There are no guidelines for the size of the domain. However, through experimenting with different domain sizes on a microscope image (90x magnification), it has been found that the SFI value decreases as the domain size increases. The SFI value will decrease to a value close to zero or form a plateau at around a small value such as 0.5 . The speed at which this decrease occurs depends strongly on the quality of the image. If the image is very clear, not blurry, and does not have any non-uniform grey areas, then there may be a plateau as depicted in Figure 47.

It is interesting to note that the analysed microscope images had a maximum SFI value between 1.5 and 2, under an initial domain size of 50 x 50 pixels $(36.23 \times 36.23)$. This corresponds well with the SFI values found from the MFIA method that uses microscope images. In the MFIA method, the domain size used was $80 \times 80$ pixels. Under a 90x magnification, this corresponds to 57.97 x $57.97 \mu \mathrm{m}$. The graph in Figure 47 was analysed under different domain sizes for one of the fourteen microscope images used for MFIA. Also, the 720 dpi and the 2400 dpi images were analysed under a domain size of 50 x 50 pixels. This is summarised in Table 4.

Table 5 summarises the SFI values found using different methods for textile 11-2 DS. For this textile, the fourteen 200 dpi microscope images were analysed using Fibre Image Analysis. Then, the SFI results from these fourteen images were consolidated using a formula similar to equation 3.3. (See Appendix B, Section I for details). For the 2400 dpi image, the SFI value is 
consolidated from four SFI values. (See Appendix B, Section II for details). Table 5 shows that the SFI values using analySIS and Fibre Image Analysis are very close to each other, with respect with the 200 dpi and the 720 dpi images. However, a significant difference can be observed with the 2400 dpi image.

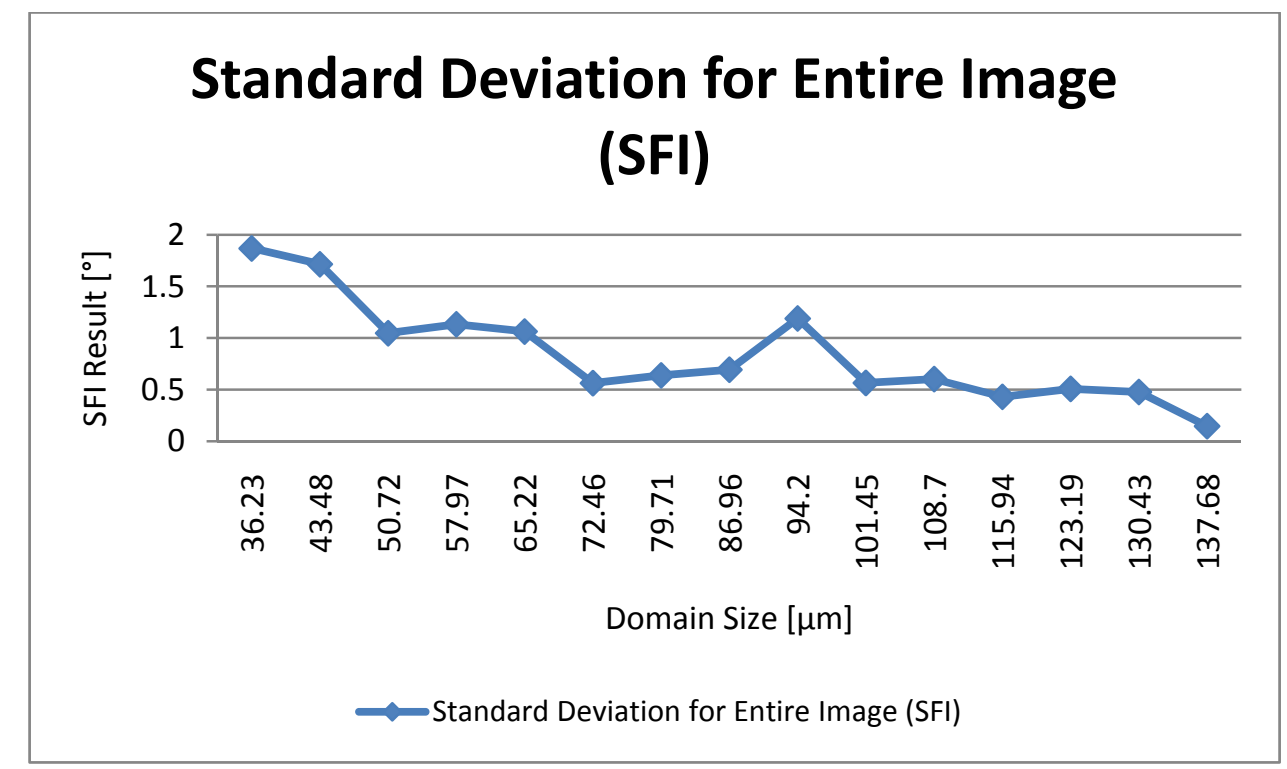

Figure 47: Analysed Microscope Image using Fibre Image Analysis, SFI versus Domain Size (textile: 11-2 (DS))

\begin{tabular}{|l|l|l|}
\hline Image & Pixel & $\boldsymbol{\mu m}$ \\
\hline Microscope & $80 \times 80$ & $57.97 \times 57.97$ \\
\hline Microscope & $50 \times 50$ & $36.23 \times 36.23$ \\
\hline 2400 dpi & $50 \times 50$ & $529.16 \times 529.16$ \\
\hline 720 dpi & $50 \times 50$ & $1763.8 \times 1763.8$ \\
\hline
\end{tabular}

Table 4: Domain Sizes

\begin{tabular}{|c|c|c|c|c|}
\hline \multirow[t]{2}{*}{ Textile } & \multirow{2}{*}{$\begin{array}{l}\text { MFIA with analySIS } \\
200 \text { dpi (M, microscope images) } \\
\text { Domain Size }: 80 \text { x } 80 \text { pixel }\end{array}$} & \multicolumn{3}{|c|}{$\begin{array}{l}\text { Fibre Image Analysis } \\
\text { Domain Size: } 50 \text { x } 50 \text { pixel }\end{array}$} \\
\hline & & 200 dpi (M) & $720 \mathrm{dpi}$ & $2400 \mathrm{dpi}$ \\
\hline $11-2(\mathrm{DS})$ & 1.541503889 & 2.533442112 & 2.304 & 4.339132778 \\
\hline
\end{tabular}

Table 5: Comparing SFI found from MFIA and Fibre Image Analysis (textile: 11-2 (DS))

The activate analysis function and the save results parameters indicate which analysis functions are activated and which results are saved. The Fibre Image Analysis software is capable of performing more than one function while displaying certain results.

By default, all activate analysis functions are activated. Currently, the parameter is set at 100010. The first digit is for activating fibre orientation. This is desired for finding the SFI, and 
is set at 1. The second digit indicates is for activating loop detection. A loop is a curved, "looped," piece of fibre, a defect, like the "fisheyes" mentioned earlier, but do not occur regularly over the entire surface of the textile. Therefore, it is not activated and is set at 0 . The third digit is for finding the width of the sample and for visualising the boundary between the sample and background. This is inactivated since it is unnecessary since the images have been cropped to remove the background. The fourth digit is for identifying the top layer and measuring its width, as in the case for mutli-layer textiles. This is also inactivated. The fifth digit is for finding the "fisheyes," called "gaps" here. This is inactivated for finding the SFI value for 2400 dpi images to save computing time, but is activated for 720 dpi images. The sixth digit is for identifying and masking markers. A silver inked marker was used to draw markers on the textile to indicate the starting position of the probe used to find the SFO, which will be discussed later. This is inactivated since all marker lines have been cropped out of the image using Corel Paint Shop Pro.

The save results parameters are all activated by default. Currently, they are set at 10101111. The first digit is for saving the results for the sample edge. The "edge" here refers to the fibre. Therefore, this is activated. The second digit refers to loops, so is inactivated. The third digit refers to gaps (fisheyes), so are activated. The fourth digit refers to markers, so is inactivated. The last four digits refer to finding the fibre orientation, through the display of four result files. So, all are activated.

The four result files that are derived from finding the fibre orientation are: fibre angle matrix, standard deviation of angles within a domain, percentage of edge points, and masking of sewing thread. Recall from Section 2.2.1.1 in Chapter 2 that there were two criteria used to avoid falsely calculated fibre orientations. The two were: 1) standard deviation of angles within a domain, and 2) percentage of edge points within a domain. Also, there was a tolerance-based warning system in place.

A large standard deviation means that the peak is very flat and there may be arbitrary angles. The maximum standard deviation parameter is used here, for criterion 1 . It is set at a default of 44 . The second fibre angle result file would show a green vector in the domain if this threshold has not been surpassed. Otherwise, a red vector would be shown in Figure 48.

A low percentage of fibre pixels in a domain means that the image region captured by the domain is: 1) blurred or 2) at the edge of the textile and part of the background is included in the 
analysis. The minimum edge percent parameter is used for criterion 2 . It is set at a default of 70 , meaning $70 \%$. This means that the domain must contain at least $70 \%$ fibre (edge). As depicted in Figure 49, if the domain contains $70 \%$ fibre, the vector in the corresponding domain in the third result file will be green. Otherwise, a red vector would be shown.

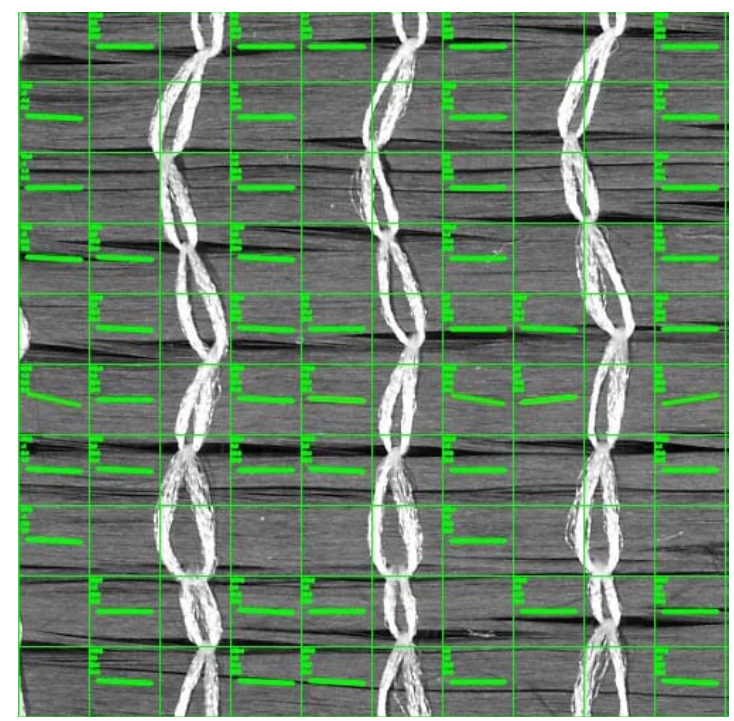

Figure 48: Fibre Image Analysis, Result File 2

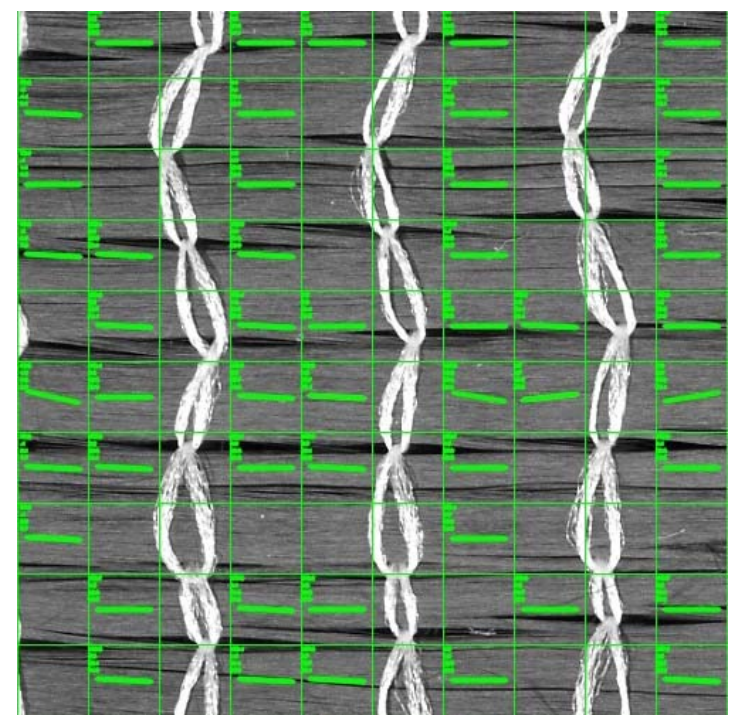

Figure 49: Fibre Image Analysis, Result File 3

Figure 50, result file 1, shows the fibre angle matrix in which a vector represents the fibre angle orientation. Because neither criterion 1 or 2 has been surpassed, which can be respectively represented by the green vectors in result file 2 and 3, none of the vectors in result file 1 are dark. However, it can be observed that there are red and yellow vectors in Figure 50 obtained from the tolerance-based warning system. Using angle Tolerances \#1 and \#2, the mean angle within the 
domain is subtracted from the mean angle of the entire image. This is required to observe the local deviations, in a domain, from the "global" fibre orientation in the whole image knowing the default value is 4 . If the resulting value is within angle Tolerance \#1, then the vector is green in the first result file. If the resulting value is between angle Tolerances $\# 1$ and \#2, then the resulting vector will be yellow. Finally, knowing the default value for angle Tolerance \#2 is 8 , if the resulting value exceeds angle tolerance 2 , then the resulting vector will be red.

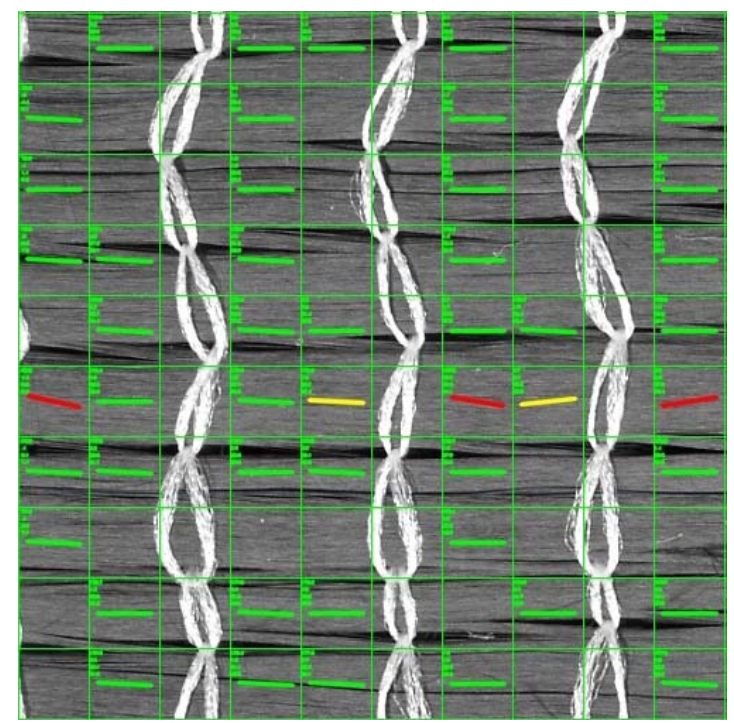

Figure 50: Fibre Image Analysis, Result File 1

As it can be observed in the result files, a portion of the domains do not have any vectors. This is because of masking. The mask window size and mask th parameters need to be adjusted to determine how the sewing thread can be correctly masked in the calculation of the fibre angles. Also, the maximum width seam millimetre parameter must be adjusted. This parameter is set at a default of 0.57 millimetres, which is the maximum width of the sewing threads. In addition to these three parameters, the minimum percent unmasked parameter is used to determine the minimum allowable percentage of the domain that is not masked for the fibre orientation calculations to occur, set at a default value of 10, representing 10\%. However, from experience, it has been concluded that this value is too low and results in erroneous fibre angle calculations in domains containing the sewing threads. Therefore, this parameter is usually set at around $90 \%$ or more to avoid fibre angle calculations to occur in domains containing the sewing thread. Figure 51 represents which parts of the image have been masked.

In addition to the image result files, there is also a resulting text file. There is possibly a fifth result image file if the detection of markers from the silver inked pen has been activated. 
The text file contains numerical results of the three aforementioned image result files: 1) fibre angle matrix, 2) standard deviation of angles within a domain, and 3) percentage of edge points. It also contains a matrix that calculates the deviation of the calculated angle in the domain from the mean angle of the entire image for the tolerance-based warnings. Also, the file contains the calculated mean fibre angle of the entire image, the SFI value, and the size of the "fisheyes" in length and width. The SFI value is calculated so that the dark vectors in the first result file are not included.

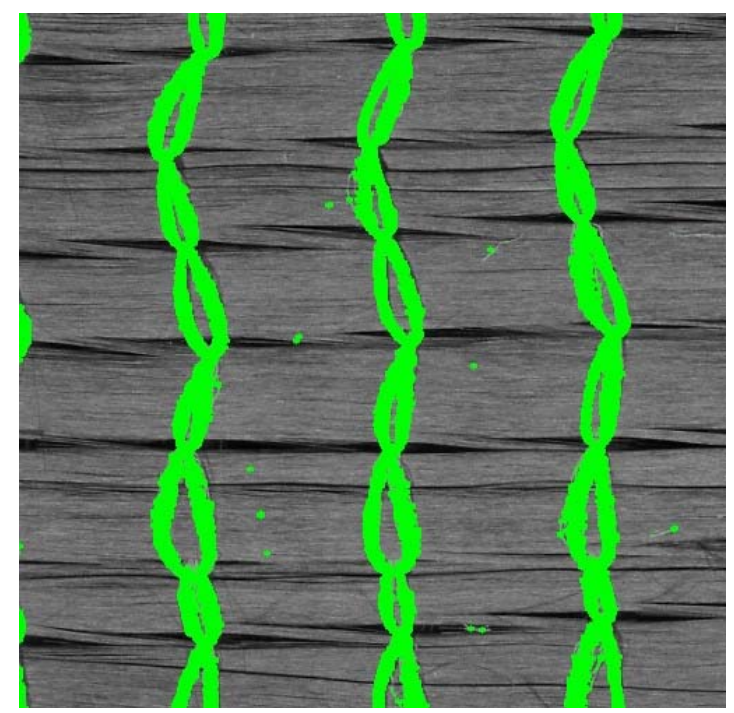

Figure 51: Fibre Image Analysis, Result File 4

In this study, 2400 dpi images were analysed using Fibre Image Analysis. The outcome shows that only images with a maximum of $9000 \times 13000$ pixels $(3.75 \times 5.42$ inches or $9.525 \times$ $13.76 \mathrm{~cm}$ ) with 2400 dpi can be analysed. This finding was found using a computer with 2038 MB RAM and a computing speed of $2.53 \mathrm{Ghz}$, but also applies to a computer with better specifications, $4096 \mathrm{MB}$ RAM and a computing speed of 3.00Ghz. Since many of the scanned images were larger than this area, the Fibre Image Analysis program would crash. To deal with this, the original 2400 dpi images were partitioned into quarters. An experiment with a computer with worse specifications, $1014 \mathrm{MB}$ RAM, $1.60 \mathrm{Ghz}$, was used to test the area that the $2400 \mathrm{dpi}$ image would need to be for the program to run. It was found that an area as small as $1 \mathrm{x} 1 \mathrm{~cm}$ $(0.39 \times 0.39 \mathrm{inch})$ would not run. It was also found that all files used should be placed on the desktop or hard drive of the computer instead of on an external drive in order for the runs to occur quickly. 
On the topic of speed, Table 6,Table 7, andTable 8 summarise the computing speed of various images, given the image's resolution and actual size, and the computer's specifications. It must be emphasized that the computing speed varies, depending on the parameter settings, the number of "fisheyes" that have been identified, and the number of result files generated. Given that the SFI and fisheyes are identified, which represents all of the files needed for this study and the maximum computing time required, it can be derived from the aforementioned tables that the computing time is generally higher for 2400 dpi images, no matter the computer's specifications. These images are denser, with more data per area.

\begin{tabular}{|l|l|l|l|}
\hline \multicolumn{2}{|l|}{ Image Size } & Memory, CPU \\
\hline Resolution & Pixels & Inches & 2038 MB RAM, 2.53 Ghz \\
\hline 720 dpi & $5586 \times 5377$ & $7.758 \times 7.468$ & $57-58$ seconds \\
\hline 2400 dpi & $5048 \times 6560$ & $2.103 \times 2.733$ & 151 seconds \\
\hline
\end{tabular}

Table 6: Image Size and Calculation Time with Fibre Image Analysis with 2038 MB RAM (textile: 14-2 (DS))

\begin{tabular}{|l|l|l|l|}
\hline \multicolumn{2}{|l|}{ Image Size } & Memory, CPU \\
\hline Resolution & Pixels & Inches & 1014 MB RAM, 1.60 Ghz \\
\hline 720 dpi & $4316 \times 5697$ & $5.994 \times 7.912$ & 307 seconds \\
\hline 2400 dpi & $4717 \times 6431$ & $1.965 \times 2.680$ & 529 seconds \\
\hline
\end{tabular}

Table 7: Image Size and Calculation Time with Fibre Image Analysis with 1014 MB RAM (textile: K1 Sh (DS))

\begin{tabular}{|l|l|}
\hline Memory, CPU & $\begin{array}{l}\text { Image Size } \\
720 \mathrm{dpi}, 5040 \text { x } 6389 \text { pixels, } 7 \times 8.874 \text { inches }\end{array}$ \\
\hline $4096 \mathrm{MB}$ RAM, 3.00 Ghz & 61 seconds \\
\hline $2038 \mathrm{MB}$ RAM, 2.53 Ghz & 61 seconds \\
\hline
\end{tabular}

Table 8: Image Size and Calculation Time with Fibre Image Analysis with 4096 MB RAM (textile: 14-2 (US))

Also, Table 8 shows that given the same image, over twofold increase in memory from 2038 RAM does not make a difference in the computing time. The computing speed is almost the same here, at $2.53 \mathrm{Ghz}$ versus $3.00 \mathrm{Ghz}$.

More studies on the computing time with respect with the image size and computing specifications would need to be performed in order to better understand what contributes to the computing time. The computing time would be a major factor in whether this process would be 
feasible in the future to be used for on-line quality control and in determining a computer's requirements for a more comprehensive analysis.

\subsubsection{Fisheyes or Stitching Yarn Distortions}

Based on the discussion in Section 3.2 in Chapter 3, the comparative analysis of "fisheyes" was performed using analySIS and using Fibre Image Analysis softwares.

To perform "fisheye" analysis using either software, the textile needs to be scanned with a flatbed scanner under a resolution of $720 \mathrm{dpi}$ and 8 bit grey scale. Some built-in filters from the scanner software (Epson) were used to make the scanned image appear brighter and have higher contrast. This is necessary since the carbon fibre textiles are naturally dark coloured and would normally appear dark and blurry in the scanned image if these adjustments were not made. A scan area of about $10 \times 10 \mathrm{~cm}$ was used. Once the image has been scanned, the same image is used for analySIS and Fibre Image Analysis.

\subsubsection{Using analySIS}

As mentioned Section 3.4.1.1 in Chapter 3, analySIS is the software packaged with the Olympus microscope used in finding the SFI with MFIA. The company that owns analySIS is "Soft Imaging Solution, Five Digital Imaging Solutions, based on analysis platform." This software is used to analyse microscope images, especially in the context of analysing particles and other surface texture of the sample under the microscope. For "fisheye" analysis, the analysis of the "fisheye" particles and other defects, as mentioned by Schneider et al. (2004), is sought. This is accomplished by specifying the analysis area of the image, using a threshold histogram to differentiate between the fibre and the sewing thread (phase analysis), definition of the classes, and finally, the analysis of the image using built-in functions used to classify the particles. An Excel output file is automatically created upon completion of analysis. The Excel file is further analysed by an Excel VBA macro to find the average length and width, in millimetres, of a "fisheye."

The average length and width of "fisheyes" is then fed into a Matlab program by Lam (2008) that has been specifically written to calculate the compression strength. This compression strength is completely based on the effect of fisheyes on the laminates. This value is divided by the compression strength of the undisturbed, ideal, laminate with completely straight fibres to find the knockdown factor. This factor is then multiplied to the compression strength value that 
was previously found using the FMB-PMB model to adjust for the effect of fisheyes on the laminates.

In SFI with Fibre Image Analysis, the sewing thread is masked. Here, the fibres are identified as a different "phase" from the sewing thread. Due to the fact that sewing threads are lighter than fibres, this can be easily accomplished through phase analysis with analySIS. Figure 52 shows a histogram of the scanned image. The green area to the left of the histogram is chosen as phase 1, the dark carbon fibres. The yellow area to the right of the histogram is chosen as phase 2, the light sewing thread. As this chosen, the image changes to green or yellow to give the user an immediate feedback on the area selected. It is important that a particular area of the image has been specified before this step is done. Otherwise, the program may not progress to analysis. It is also helpful to experiment with smaller areas of the image and progress to analysis to see if these settings have been set correctly.

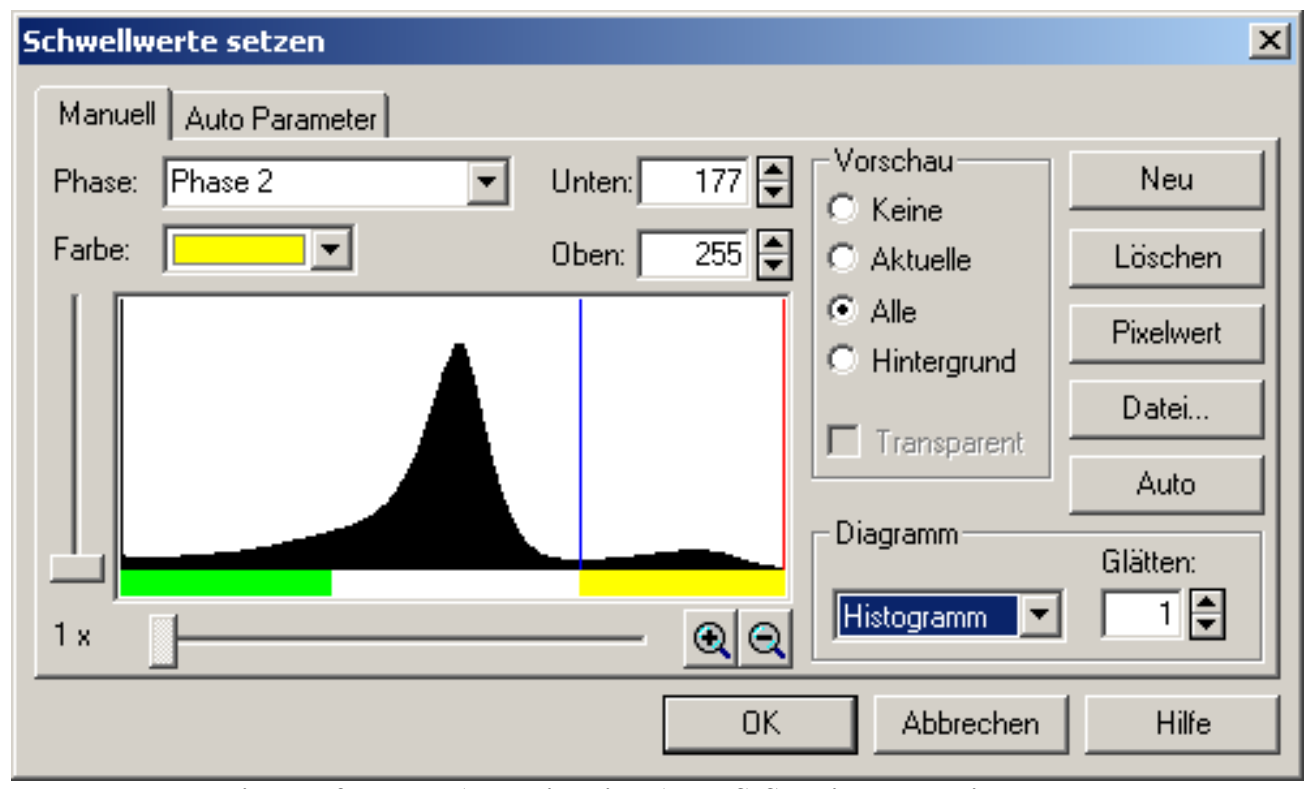

Figure 52: Phase Analysis using AnalySIS, Brightness Histogram

If the fibre and the sewing thread have been selected correctly, the derivation graph of the histogram would be at zero at the end point of phase 1 and at the start point of phase 2 as shown in Figure 53.

Next, different classes of particles are defined based on a built-in function in analySIS. One of these functions is the Phase Name that was previously described. The criterion used to classify particles is Extension Exterior Min (Ausdehnung Innen Min). This function takes the 
minimum length of the line formed within the particle, passing through its centre, given that every angle has been tried, as depicted in Figure 54.

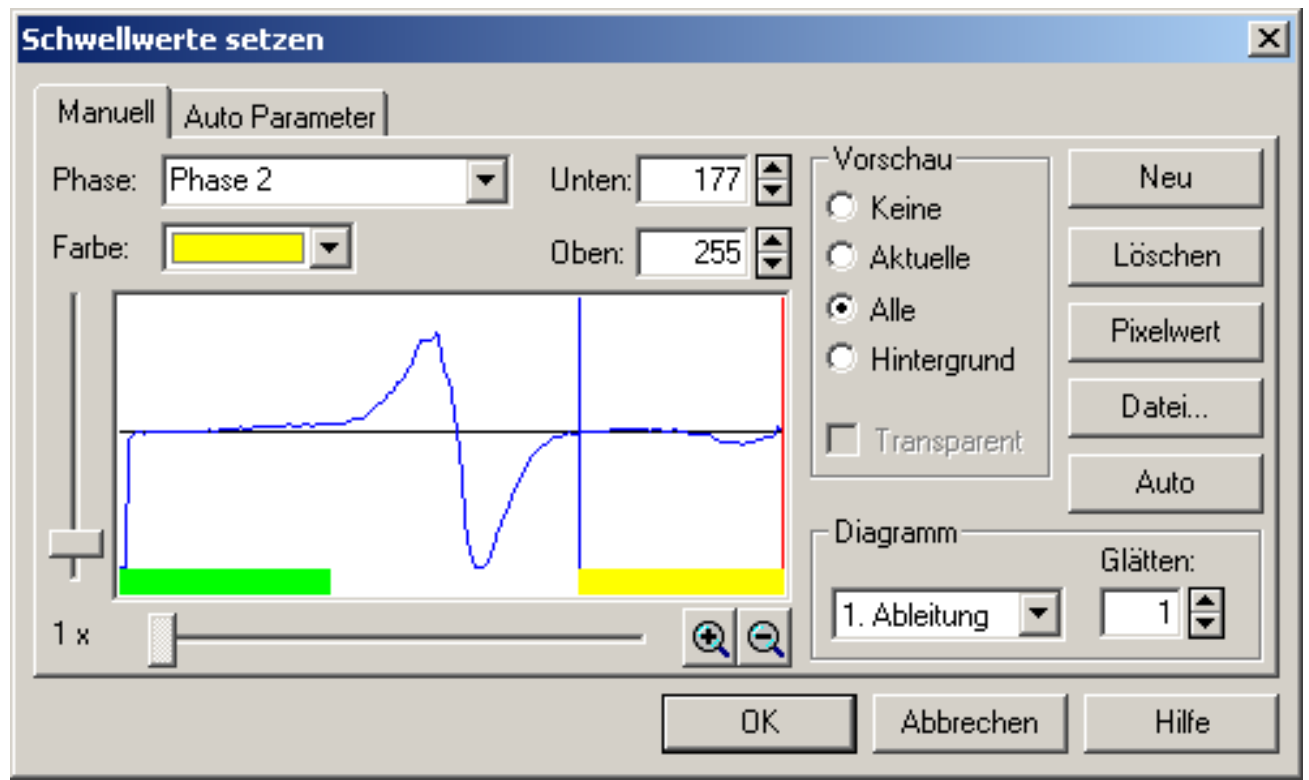

Figure 53: Derivation Graph, Derivation of Brightness Histogram

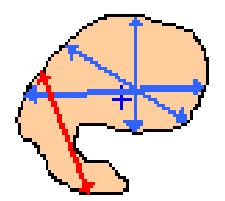

Figure 54: Extension Interior Min (Ausdehnung Innen Min)

Using the length found from Extension Interior Min, the Class Name function can be used to classify the particles. The particles were classified according to Table 9. In addition to these four functions for classifying and naming the particles, four other functions are used to give four other types of information about each particle: Area, Orientation, Diameter Max, and Feret Min. The area is the area of the particle in $\mathrm{mm}^{2}$. The diameter max is the maximum diameter of the particle in millimetres. The orientation is the orientation of the particle in degree $\left(^{\circ}\right)$ that is defined as the angle formed between the maximum diameter line of the particle and the $x$-axis. Feret Min is the minimum length of the line formed by parallel tangents on the border of the particle as shown in Figure 55. 


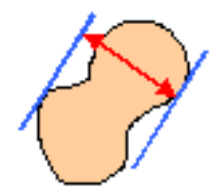

Figure 55: Feret Min in analySIS

The analySIS software creates an Excel file upon completion of the analysis of an image. Inside the file is a list of all particles found and their respective categories and measurements. With this file, further analysis can be performed by using Excel VBA macros. Based on different combinations of if-then rules that involve particle Phase Name, Extension Interior Min length, Class Name, Area, Orientation, Diameter Max, and Feret Min, the particles are further classified as: fuzzball, splice, and sewing thread. Here, a fuzzball is a particle with an area greater than 100 $\mathrm{mm}^{2}$ and a splice is a particle with an area between $10 \mathrm{~mm}^{2}$ to $100 \mathrm{~mm}^{2}$. Also, for the particles listed in Table 9, the standard deviation of the width, length, area, and orientation of the particles were calculated, and the count, average, and maximum length of the particles were derived. New classifications of the particles were based on these values.

\begin{tabular}{|l|l|l|}
\hline Extension Interior Min Length (mm) & Classification & ID Class \\
\hline 0.00 to 0.10 & Filament-Gap & 1 \\
\hline 0.10 to 0.70 & Fisheye & 2 \\
\hline 0.70 to 1.50 & Gap 0 & 3 \\
\hline 1.50 to 2.00 & Gap 1 & 4 \\
\hline 2.00 to 15.00 & Gap 2 & 5 \\
\hline
\end{tabular}

Table 9: Classification of Particles in analySIS

Of particular interest of this classification method is the average length and width of fisheyes and the average width $\left(\mathrm{w}_{\mathrm{y}}\right)$ of the sewing thread. These values are required for the next step, in the Matlab program, which will be discussed later. Since the "fisheyes" are separated by the sewing thread, the following formula is used to calculate the actual average length $\left(\ell_{\mathrm{F}}\right)$ of the "fisheye," given that the average length of the half "fisheyes" $\left(\ell_{H}\right)$ have been found. See Figure 56 for a visual explanation.

$$
\ell_{\mathrm{F}}=2 \cdot \ell_{H}+\mathrm{w}_{\mathrm{y}}
$$




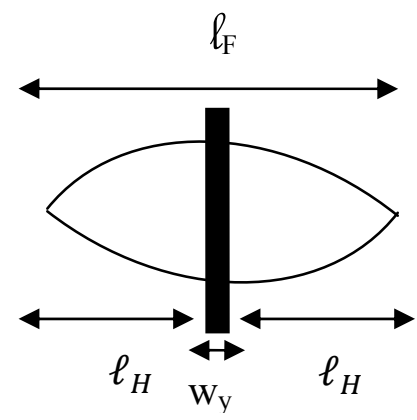

Figure 56: Calculation of Average Length of "Fisheye"

The Excel VBA macro was originally designed for only two types of textiles. These two textiles were relatively neat and had clearly identifiable "fisheyes" as shown in Figure 57. Here, the green particles are "fisheyes." They have a noticeably regular pattern with a horizontal orientation. Figure 58 shows a textile that did not have such clear and identifiable "fisheyes." Also, as mentioned in Section 3.3 in Chapter 3, some of the textiles were sheared. Therefore, further developments were made, by the author, on the Excel VBA macro to account for the additional requirements needed to identify and quantify "fisheyes" in sheared textiles, especially those that did not have clearly identifiable "fisheyes."

One of the problems with analysing sheared textiles, especially those textiles that originally do not have clear identifiable "fisheyes," is that there are many dark, curved pieces of fibre that are identified as "fisheyes" with analySIS, as shown in the many curved green particles in Figure $58 \mathrm{~d}$. Since numerous attempts to filter out these erroneous classifications failed using analySIS, these erroneous classifications were filtered out from the Excel result file using a modified Excel VBA macro. 


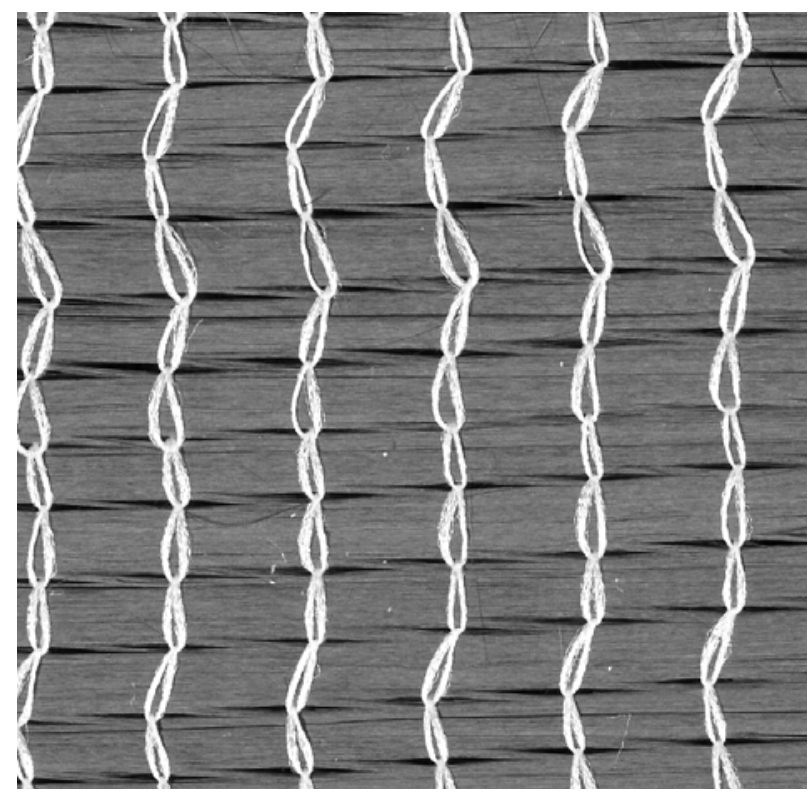

a)

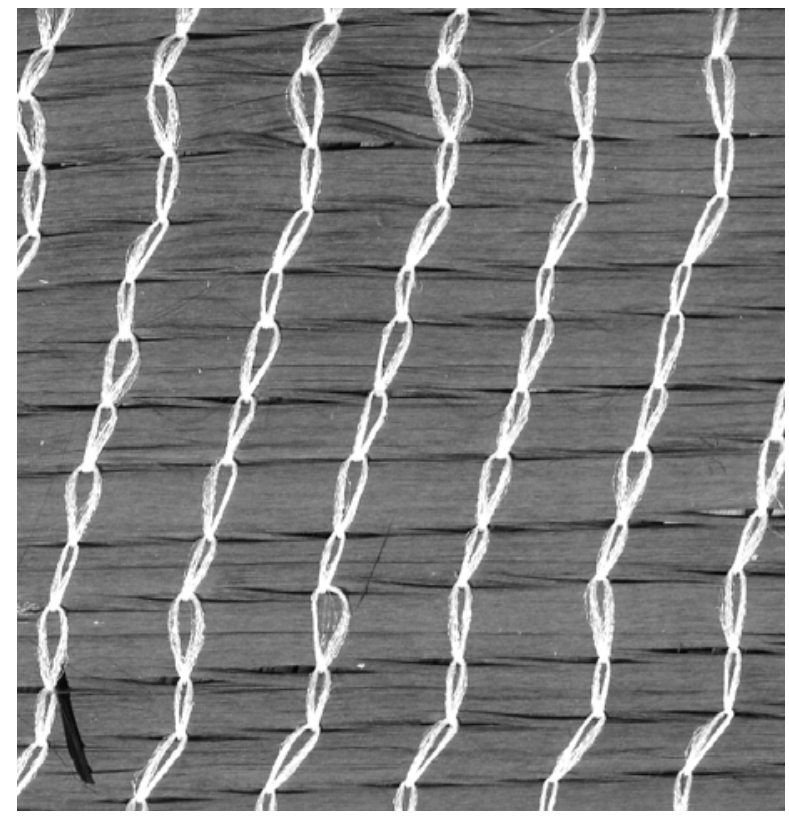

c)

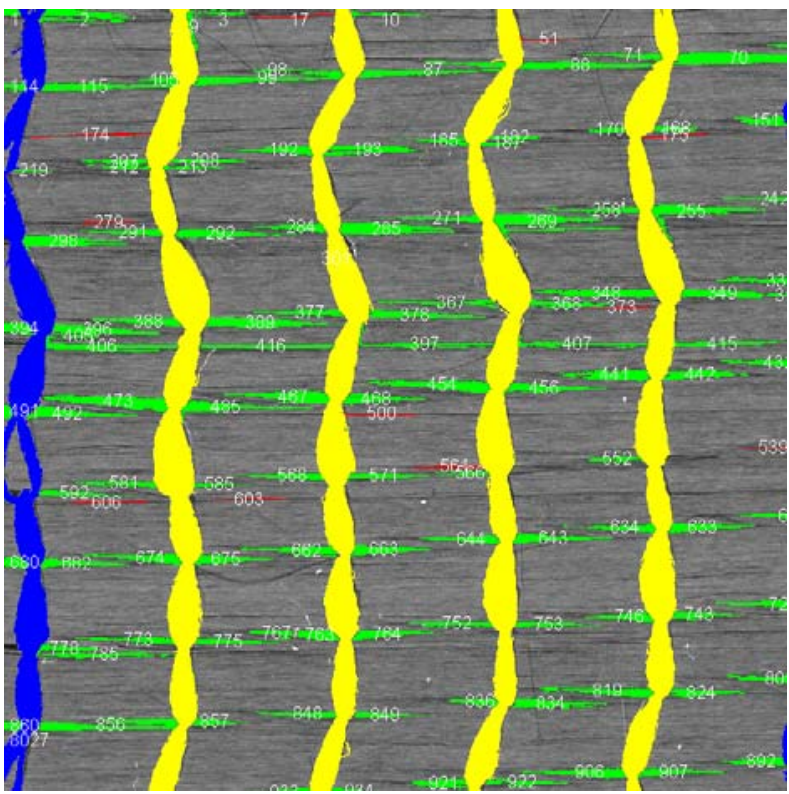

b)

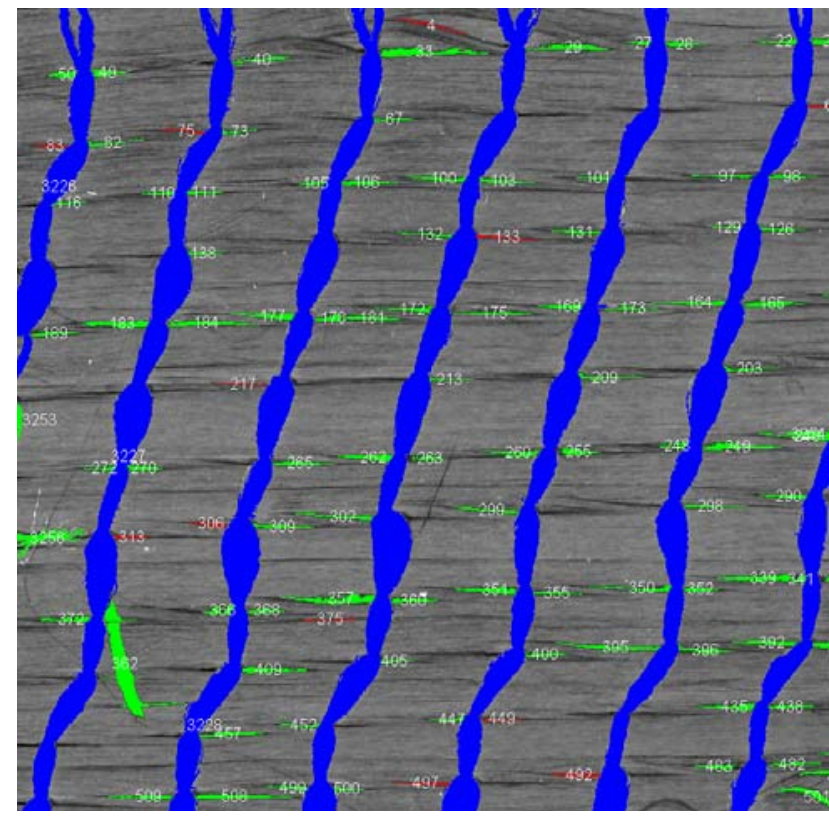

d)

Figure 57: Textile 11, Clear Identifiable "Fisheyes" a) Original Textile b) Original Textile after Particle Analysis c) Sheared Original Textile d) Sheared Original Textile after Particle Analysis 


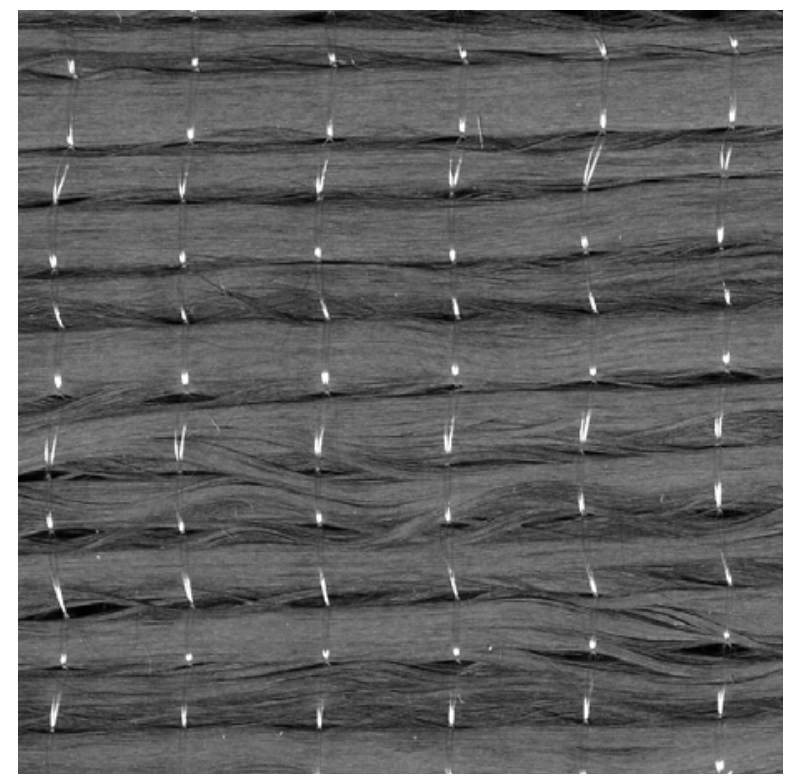

a)

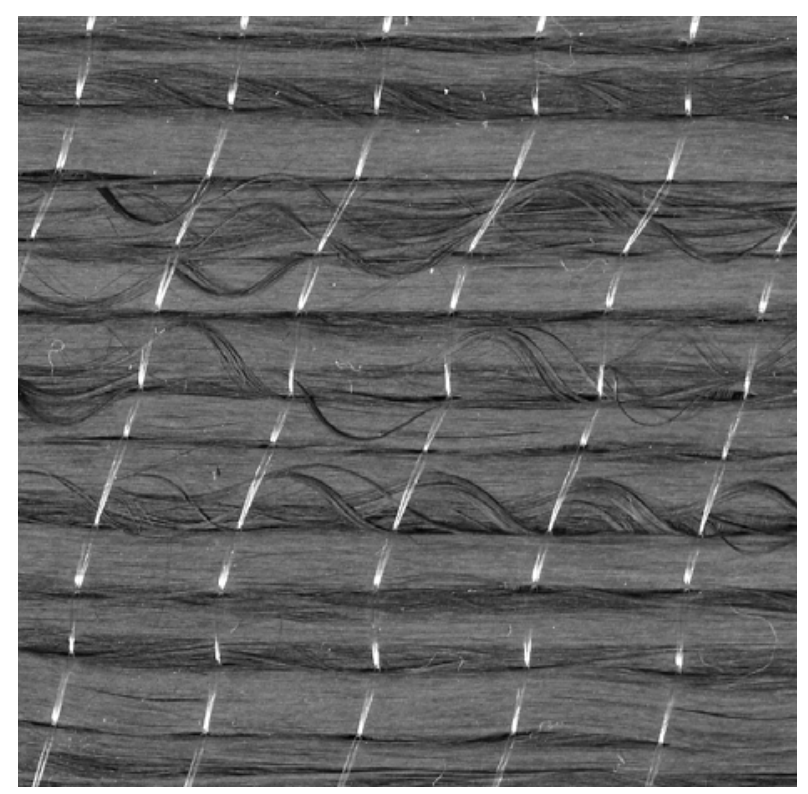

c)

Figure 58: Textile KMV, Not Clear Identifiable "Fisheyes" a) Original Textile b) Original Textile after Particle Analysis c) Sheared Original Textile d) Sheared Original Textile after Particle Analysis

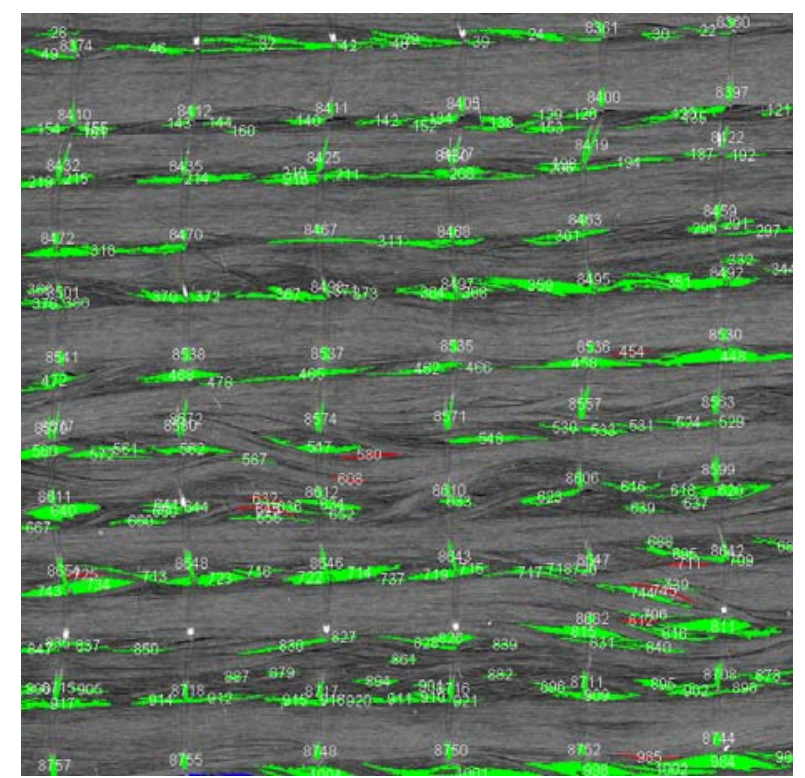

b)

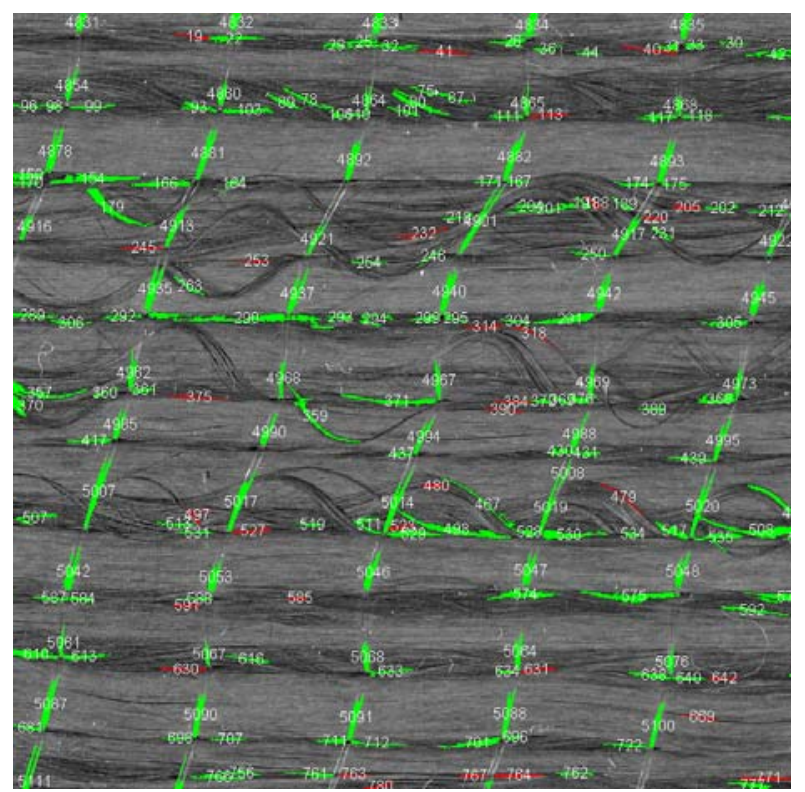

d)

The modified Excel VBA macro uses the value of Feret Min - Extension Interior Min (Ausdehnung Innen Min) as a filtering criterion. Feret Min is defined as "minimum distance of parallel tangents at particle borders." Extension Interior Min is defined as "the minimum of all angle specific, calculated length, which lie completely within the particle." Therefore, it can be hypothesized that the Feret Min will be larger than the Extension Interior Min for curved pieces of fibre that have been mistaken as fisheyes. In contrast with actual fisheyes, the Extension 
Interior Min and the Feret Min should be the same, with local differences due the pixelation of the acquired fisheye particle, as shown in Figure 59.

\section{Expected shape of "fisheye"}

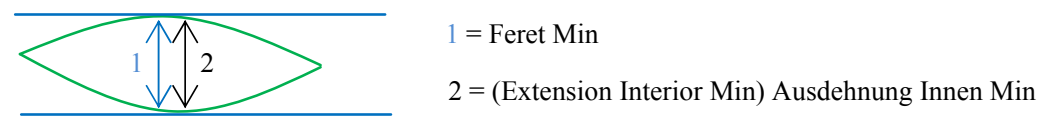

\section{Pixelation of the acquired "fisheye" (reality)}

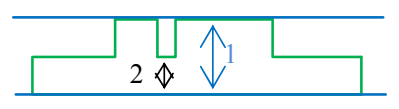

\section{Curved Fibres}

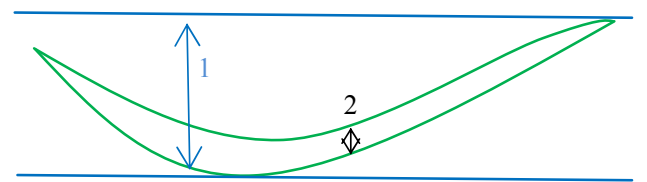

Figure 59: Feret Min - Extension Interior Min (Ausdehnung Innen Min)

In the case with curved fibres due to shearing, Feret Min - Extension Interior Min results in a positive value for erroneous "fisheye" classifications. This is particularly true for curved fibres, where the difference will be greater than straight fibres. Therefore, an additional if-then statement is written such that the difference between Feret Min and Extension Interior Min must be less than a particular value, in order for a particle to be considered to be a "fisheye." Since the "threshold value" may vary on the textile, the value may need to be adjusted accordingly. In this study, this "threshold value" was found by analysing a scatter plot and a cumulative histogram of the values of Feret Min - Extension Interior Min of all of the particles originally classified as "fisheyes." The "threshold value" was taken as the value that encompasses roughly 80 to $90 \%$ of all of the particles, thus successfully removing extreme differences. The Feret Min Extension Interior Min values for the textiles are listed in the Table 10. 


\begin{tabular}{|c|c|c|c|}
\hline \multirow[t]{2}{*}{ Textile } & \multicolumn{2}{|c|}{ Diameter Max } & \multirow{2}{*}{$\begin{array}{l}\text { Feret Min - Extension Interior } \\
\text { Min less than (mm) }\end{array}$} \\
\hline & $\operatorname{Min}(\mathrm{mm})$ & $\operatorname{Max}(\mathrm{mm})$ & \\
\hline K1-1 (DS) & 0.7 & 5 & 0.2 \\
\hline K1-1 (US) & 1 & 20 & 0.2 \\
\hline K1-2 (DS) & 0.7 & 5 & 0.1 \\
\hline K without Trikot 1 (DS) & 1 & 5 & 0.1 \\
\hline $\mathrm{K}$ without Trikot 2 (DS) & 1 & 5 & 0.1 \\
\hline K with Trikot 1 (DS) & 1.5 & 5 & 0.15 \\
\hline K with Trikot 2 (DS) & 1 & 5 & 0.1 \\
\hline 14-1 (DS) & 0.9 & 5 & 0.15 \\
\hline 14-1 (US) & 0.9 & 5 & 0.15 \\
\hline $14-2(\mathrm{DS})$ & 1.2 & 5 & 0.1 \\
\hline $14-2$ (US) & 1 & 5 & 0.1 \\
\hline $11-1(\mathrm{DS})$ & 1.5 & 5 & 0.2 \\
\hline $11-1$ (US) & 1.2 & 5 & 0.1 \\
\hline $11-2$ (DS) & 1.7 & 5 & 0.15 \\
\hline $11-2$ (US) & 1.7 & 5 & 0.2 \\
\hline SQ No Yarn & 2 & 5 & 0.05 \\
\hline SQ with Yarn & 1 & 3 & 0.09 \\
\hline 397 (DS) & 0.8 & 2.8 & 0.13 \\
\hline 397 (US) & 1 & 4 & 0.09 \\
\hline 399-1 (DS) & 0.9 & 3 & 0.1 \\
\hline 399-2 (DS) & 0.8 & 5 & 0.1 \\
\hline 400-1 (DS) & 0.8 & 2.5 & 0.1 \\
\hline $400-2$ (DS) & 0.9 & 3 & 0.13 \\
\hline $401-1$ (DS) & 0.9 & 3 & 0.13 \\
\hline $401-2$ (DS) & 0.8 & 3 & 0.13 \\
\hline K1 Sch (DS) & 1 & 5.5 & 0.2 \\
\hline 11-1 Sh (DS) & 1 & 5.5 & 0.16 \\
\hline 11-1 Sh (US) & 1 & 2.5 & 0.1 \\
\hline 14-1 Sh (DS) & 1 & 5.5 & 0.4 \\
\hline 14-1 Sh (US) & 1 & 2.5 & 0.1 \\
\hline 399-1 Sh (DS) & 1 & 5 & 0.1 \\
\hline $400-1 \mathrm{Sh}$ (DS) & 1 & 5 & 0.1 \\
\hline 401-1 Sh (DS) & 1 & 5 & 0.1 \\
\hline
\end{tabular}

*Some of the above textiles were not further analysed for compression strength

Table 10: Filters for using analySIS for "Fisheye" analysis

In addition to the Feret Min - Extension Interior Min criterion, two other criteria were also added as if-then conditions to avoid erroneous classifications: Diameter Max and Orientation. The maximum diameter of the "fisheyes" were analysed to find upper and lower limits that encompass $80 \%$ to $90 \%$ of all of the particles, a scatter plot, and a cumulative histogram. The 
particle orientation needs to be normalised by shifting it by $180^{\circ}$ if the measured orientation was less than $90^{\circ}$. This is necessary because all measured orientations will centre at either $0^{\circ}$ or $180^{\circ}$, and leaving a large empty range without any values. As a result, only particles with Feret MinExtension Interior Min value less than a threshold or the normalised orientation greater than $170^{\circ}$ and less than $270^{\circ}$ and are within a diameter range encompassing $80 \%$ to $90 \%$ of the particles are taken as "fisheyes."

\subsubsection{Using Fibre Image Analysis}

Fibre Image Analysis is also capable of analysing the scanned image for "fisheyes." This analysis was performed only for $720 \mathrm{dpi}$ images. This is accomplished by adjusting the following parameters, as found from the experience of the author: gap window size, gap threshold, gap global threshold, gap maximum angle difference, gap melt distance seam millimetre, gap melt distance filament millimetre, gap minimum width millimetre, gap maximum width millimetre, gap minimum length millimetre, and gap maximum length millimetre.

Just like mask window size and mask threshold that were previously mentioned in Section 3.4.2.1 in Chapter 3, the gap window size, gap threshold, and gap global threshold can be adjusted to detect the gaps or "fisheyes." By default, gap window size is set at 151 and gap threshold is set at 50. If the gap threshold value is changed to a smaller value, more pixels will be classified as a gap or "fisheye." Care needs to be given to selecting the window size. It should be selected with respect with the image resolution. If the window is too small, only the outline of the "fisheye" will be classified as "fisheye." Also, the gap global threshold value is used to compensate for small values of gap window size, which would result in small holes in a large gap region. Pixels that are darker and smaller than a threshold value are grouped together. The gap global threshold value is set at a default of 0 . However, it is better to set it at 10 for scanned images.

Setting the default value at 10, the gap maximum angle difference is the maximum angular deviation between the fibre orientation and a straight line that approximates the orientation of the "fisheye."

The gap melt distance seam millimetre and gap melt distance filament millimetre parameters are used to determine the acceptable distances, in millimetres, to "melt" or include as "fisheye," when two "fisheyes" are separated by an object classified as a seam or sewing thread 
or a filament of fibre. By default, it is acceptable to "melt" two "fisheyes" together separated by a sewing thread thickness of $2 \mathrm{~mm}$ or a filament thickness of $0.2 \mathrm{~mm}$. Since some sewing threads are very thin, $2 \mathrm{~mm}$ may cause a very large number of erroneous "fisheyes" to be detected. It is also possible that the carbon fibre filament is thinner or thicker than $0.2 \mathrm{~mm}$. Therefore, this parameter would also need to be adjusted accordingly.

The gap minimum width millimetre, gap maximum width millimetre, gap minimum length millimetre, and gap maximum length millimetre parameters adjust the minimum and maximum width and length of particles classified as "fisheyes." By default, the minimum gap width is set at $0.6 \mathrm{~mm}$ and the maximum gap width is set at -1 , which means no limit. Also, the minimum gap length is set at $4 \mathrm{~mm}$ and the maximum gap length has no limit. The width is defined as the widest point of the particle and the length is measured parallel to the fibre. These default values have to be adjusted to better suit each textile.

The resulting text file lists the width and length of each "fisheye" particle found. Also, the average width and length of all "fisheye" particles are listed in an Excel file.

\subsubsection{Parametric Study with the Unit Cell Model}

As discussed in Section 3.2 in Chapter 3, the Unit Cell model (Heß, Roth, \& Himmel, 2007), as discussed in Section 2.4 in Chapter 2, a model that was developed by Institut für Verbundwerkstoffe (IVW) (Institute of Composite Materials) based at the TU Kaiserslautern (University of Kaiserslautern), is a FE-Model used to simulate the influence of stitching: the length, width, stitch distance, and stitch length of "fisheyes." A part in the Diploma thesis of Lam Pham (Lam, 2008) is devoted to the estimation of compression strength values through linear interpolation and extrapolation and cubic interpolation between known values found from the Unit Cell Model based on a FVC of $60 \%$. Lam's model was created in Matlab and is beneficial since it would eliminate time consuming FE simulations given that the database of known values has been carefully chosen. This model was not modified. The author only used this model and the software.

Lam's model uses four variables as input that results in four output values. The four input variables are: stitch distance $(s)$, stitch length $(p)$, average width of "fisheye" $(b)$, and average length of "fisheye" $(\ell)$. The four output values are: axial (in-fibre) modulus $\left(E_{l}\right)$, transverse 
(perpendicular) modulus $\left(E_{2}\right)$, axial shear modulus $\left(G_{12}\right)$, and compression strength (in-plane) $\left(R_{l}\right)$. Only the compression strength (in-plane) is needed for this study.

The stitch distance is defined here as the distance, in millimetres, between successive needle punctures into the textile, perpendicular to the fibre direction of the respective fibre ply. The stitch length is the distance in fibre direction of the respective ply as shown in Figure 60. The stitch distance and stitch length could be found in the textile manufacturer's files or through direct measurement with analySIS. The average width and length of a fisheye was found using analySIS or Fibre Image Analysis, as discussed previously.

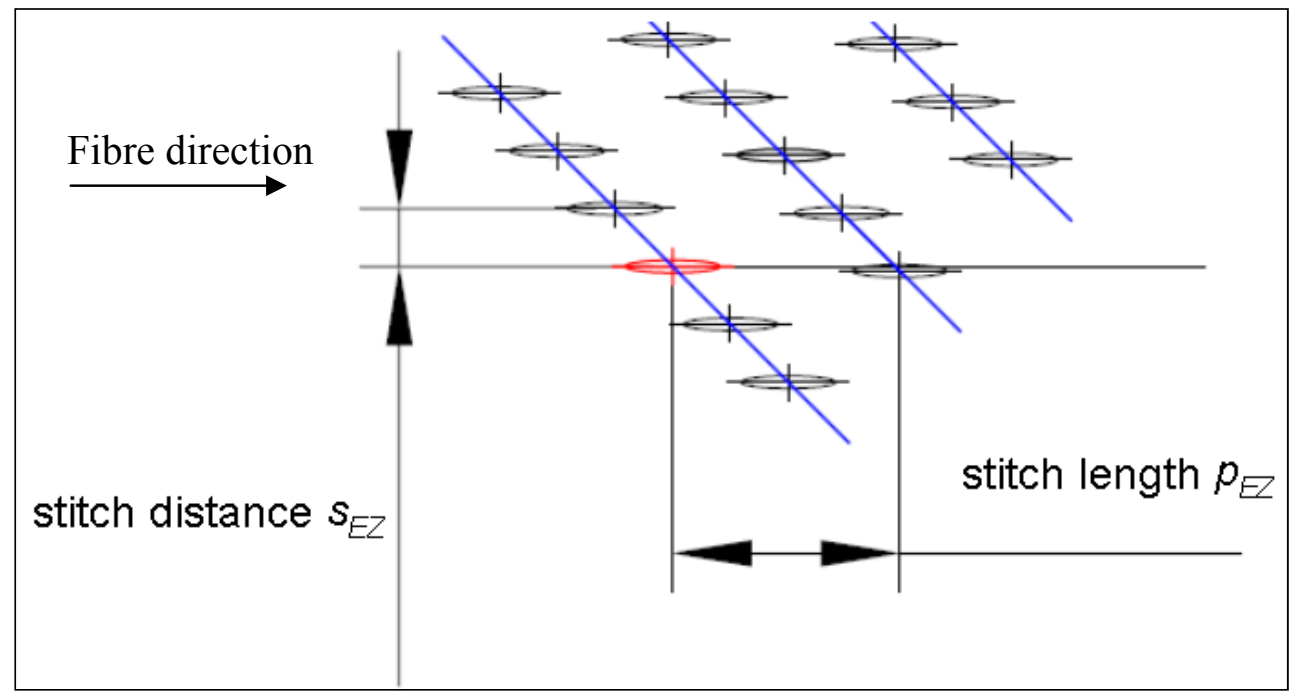

Figure 60: Parameters of Unit Cell Model (Rehbein, 2009)

The database of values created for interpolation and extrapolation is based on the stitch distance $(s)$ having values of $1.8,2.6,3.8$, or $5 \mathrm{~mm}$; the stitch length $(p)$ having values of 2.6, 3.8, 5 , or $7 \mathrm{~mm}$; the average "fisheye" width $(b)$ having values of $0.25,0.4,0.5$, or 0.8 ; the average "fisheye" length $(\ell)$ having values of 3 or $7 \mathrm{~mm}$. Each combination of these values results in a corresponding value for axial (in-fibre) modulus $\left(E_{1}\right)$, transverse (perpendicular) modulus $\left(E_{2}\right)$, axial shear modulus $\left(G_{12}\right)$, and compression strength (in-plane) $\left(R_{l}\right)$, found from the Unit Cell Model, creating a database of values. Also, the situation when $s, p, b$, and $\ell$ are all zero is included in the database. This situation occurs when the laminate is "undisturbed" or has the ideal configuration of perfectly straight fibres without any "fisheyes."

Given an input of $s, p, b$, and $\ell$ into the Matlab program, the program first performs computations with the input values to determine if the input makes sense (if the input is 
completely empty?) and if there are any missing values in the input and in the existing database matrix. Missing values in the input and missing input values in the existing database are linearly (polynomial) interpolated or extrapolated from existing values, and their corresponding missing output values: $E_{1}, E_{2}, G_{12}, R_{1}$ are found. As a result, a new database matrix is created that includes all missing values. With this new matrix, cubic interpolation is performed based on the input parameters to find the output values. It is also important to emphasize here that only one of the input values can be extrapolated. If there is more than one, then the program will return "NaN," meaning "Not a Number." This situation occurs when two of the input values are below the minimum values.

Using the compression strength (in-plane) $\left(R_{1}\right)$, it is divided by the compression strength of an undisturbed laminate, which would be greater than $R_{l}$. The resulting value, a decimal value between zero and one, is the "knockdown factor" due to "fisheyes." The knockdown factor is multiplied to the compression strength value predicted by the FMB-PMB model to take into account for the effect of "fisheyes" in the textile. The FMB-PMB model only takes into account the SFI, in-plane fibre misorientations, and the SFO, the out-of-plane waviness of the textile, which will be explained in the next Section, 3.5.

\subsection{SAMPLE STANDARD DEVIATION OF FIBRE OUT-OF-PLANE (SFO)}

The out-of-plane waviness of a textile can be by using a perthometer that runs a probe across the surface of the textile, and the deflections of the probe measured and recorded as shown in Figure 62. The method, devised by Kuntz (2010), involves taking a piece of MWK fabric and placing it on a metal base (caul) plate. Then, the plate and fabric assembly is enclosed it in a thin polyethylene vacuum foil sealed with mastic tape. Air is removed from the assembly with a pump until a pressure close to 0 bar is reached. As a result, the textile is pressed down, revealing the textile structure, and making it easy for the measurements to take place, as shown in Figure 61.

The probe is run across the surface of the compacted textile, on both sides of the textile: the Up Side and the Down Side. For each side, the probe is run in-fibre direction and also perpendicular to fibre direction, as depicted and Figure 62. On each side of the textile, 10 runs are made, where five were in the in-fibre direction, and five in the perpendicular to fibre direction. Runs in the $90^{\circ}$ direction are extra measurements taken for verification purposes. The 
runs were equally spaced from each other, with their starting points marked with a silver inked marker.

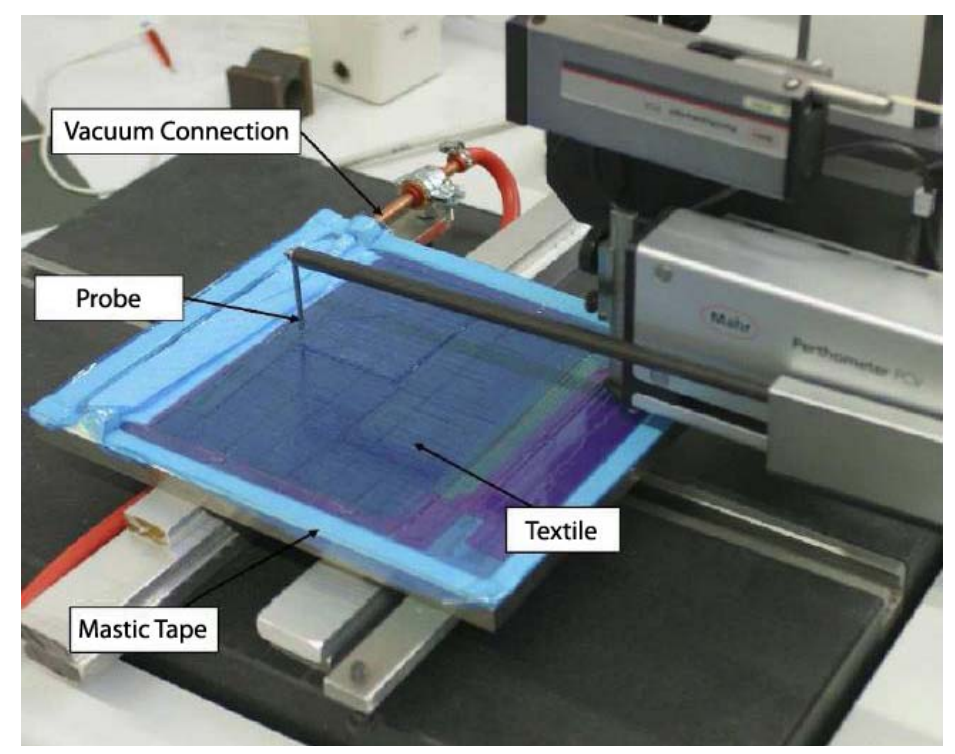

Figure 61: Measurement of Waviness using a Perthometer manufactured by Mahr

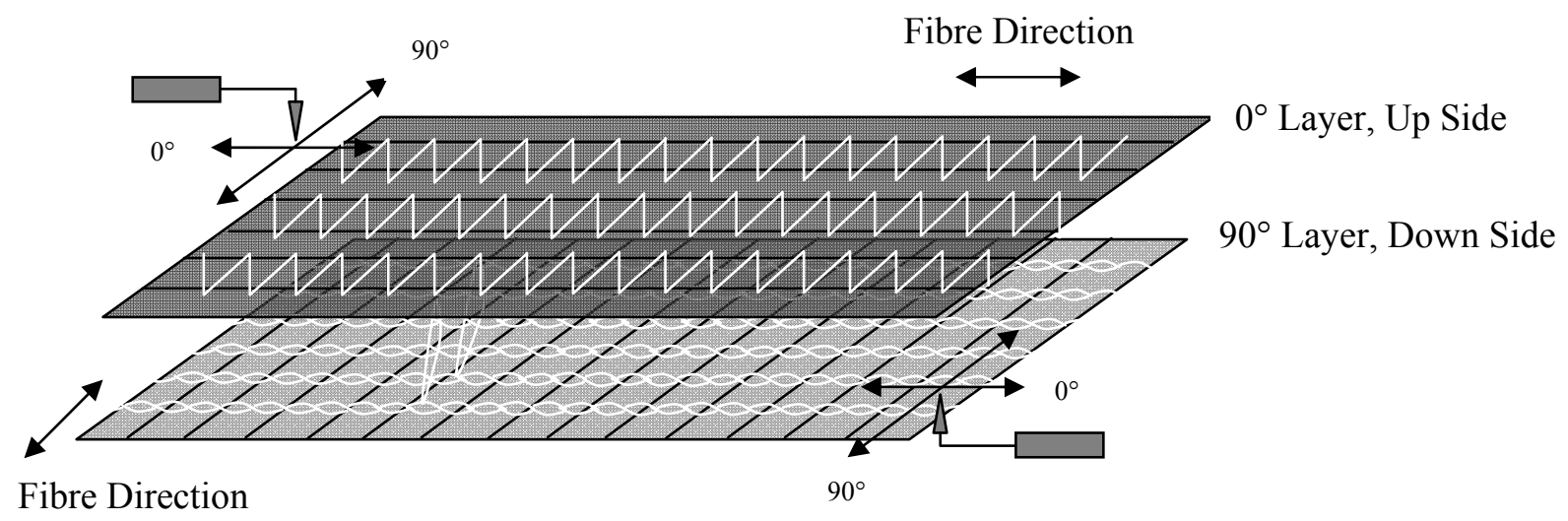

Figure 62: Measurement of SFO (Up Side, Down Side)

\subsubsection{Analysis of Collected Waviness Data}

The analysis was performed by the author, using an MS Excel file that contained the VBA code developed by Lehners (2008). The author modified the original VBA code to deal with lines of code that were skipped due to large file size and insufficient computer memory. 
For each run of the probe across the textile surface, the horizontal distance $(X)$ travelled and the vertical position $(Z)$ deflected are measured and recorded. These results are imported into an Excel spreadsheet and further analysis is performed using Excel VBA macros, using Fast Fourier Transform (FFT). According to Lehners (2008), the macros first convert the results, originally in micro-metres $(\mu \mathrm{m})$, into millimetres. Then, the $X$ values are normalised. See equation 3.6. This is necessary because there is an offset distance from the machine's zero level and the measurements.

$$
\mathrm{x}_{\mathrm{i}, \text { norm }}=\frac{\mathrm{x}_{\max }-\mathrm{x}_{\min }}{\mathrm{N}-1} \cdot \mathrm{i} \quad \text { For } \mathrm{i}=0,1, \ldots, \mathrm{N}-1
$$

Where, $\mathrm{x}_{\max }$ and $\mathrm{x}_{\min }$ are the maximum and minimum $x$ values, $N$ is the number of all measured points, and $i$ is the $i^{\text {th }} x$ value. The readings were taken at equally spaced intervals.

The offset between the machine zero level and the measurements were not only affected in the $X$ direction, but also in the $Z$ direction. Correspondingly, the $Z$ values are normalised according to equation 3.7. However, opposed to the original $X$ values, the original $Z$ values have a slight erroneous slope. Therefore, a regression line, similar to $m x+b$, is subtracted from the original $Z$ values.

$$
z_{i, \text { norm }}=z_{i}-\left(c_{R, 1} x_{i}+c_{R, 0}\right) \quad \text { For } \mathrm{i}=0,1, \ldots, \mathrm{N}-1
$$

The regression coefficients, $c_{R, 1}$ and $c_{R, 0}$ are calculated form least squares method, as defined by equations 3.8 and 3.9 .

$$
\begin{aligned}
c_{R, 1}= & \frac{\mathrm{N} \sum_{\mathrm{i}=0}^{\mathrm{N}-1} \mathrm{x}_{\mathrm{i}} \mathrm{z}_{\mathrm{i}}-\left(\sum_{\mathrm{i}=0}^{\mathrm{N}-1} \mathrm{x}_{\mathrm{i}}\right)\left(\sum_{\mathrm{i}=0}^{\mathrm{N}-1} \mathrm{z}_{\mathrm{i}}\right)}{\mathrm{N} \sum_{\mathrm{i}=0}^{\mathrm{N}-1} \mathrm{x}_{\mathrm{i}}^{2}-\left(\sum_{\mathrm{i}=0}^{\mathrm{N}-1} \mathrm{x}_{\mathrm{i}}\right)^{2}}=\frac{\operatorname{cov}(\mathrm{x}, \mathrm{z})}{\operatorname{var}(\mathrm{x})} \\
c_{R, 0} & =\frac{\sum_{\mathrm{i}=0}^{\mathrm{N}-1} \mathrm{z}_{\mathrm{i}}}{\mathrm{N}}-c_{R, 1} \frac{\sum_{i=0}^{N-1} x_{i}}{N}=\bar{z}-c_{R, 1} \bar{x}
\end{aligned}
$$


Next, a Fast Fourier Transform (FFT) is used to detect and to remove periodic "noise" signals and to identify the origins of the textile waviness. Excel has an in-built FFT data analysis function. However, this function can only analyse up to 4096 sampling points $(F)$ and total data points must be in the power of two. Since the number of measurements is around 25000 measurement points depending on the length of the textile measured, the measured data is down sampled according to equations 3.10 and 3.11 .

$$
\begin{aligned}
& \mathrm{x}_{\mathrm{i}, \mathrm{al}}=\mathrm{x}_{\mathrm{a} \cdot \mathrm{i}, \mathrm{norm}} \\
& \mathrm{z}_{\mathrm{i}, \mathrm{al}}=\mathrm{z}_{\mathrm{a} \cdot \mathrm{i} \text {,norm }} \quad \text { For } \mathrm{i}=0,1, \ldots, \mathrm{F}-1
\end{aligned}
$$

Here, $F$ is the maximum number of sampling points, used in the FFT function in Excel, which is 4096 , and " $a$ " is the aliasing factor. All $i^{\text {th }} x$ and $z$ values are omitted except every $a^{\text {th }}$ value starting at $a=0$. The result is a filtered profile, a profile with smoother peaks, of the graph that represents the deflections of the probe over the distance travelled.

Assuming that the analysed data is periodic, the Fourier coefficients, in the complex form of $a+b i$, are:

$$
\hat{\mathrm{z}}_{\mathrm{i}}=\sum_{\mathrm{k}=0}^{\mathrm{F}-1} \mathrm{z}_{\mathrm{k}, \mathrm{al}} \cdot \mathrm{e}^{-\mathrm{j} \frac{2 \pi}{\mathrm{F}} \mathrm{ki}} \quad \text { For } \mathrm{i}=0,1, \ldots, \mathrm{F}-1 \text { and } \mathrm{j}^{2}=-1
$$

Where, $1+F / 2$ independent coefficients are obtained.

Next, equation 3.12 is used in the following equation, an approximation of the real data for an interval of $\left[0, \mathrm{x}_{\mathrm{F}-1, \mathrm{al}}\right]$ :

$$
\mathrm{z}(\mathrm{x})=\frac{\mathrm{Z}_{0}}{2}+\sum_{\mathrm{i}=1}^{\mathrm{F} / 2} \mathrm{Z}_{\mathrm{i}} \cos \left(\frac{2 \pi \mathrm{i}}{\mathrm{x}_{\mathrm{F}-1, \mathrm{al}}} \mathrm{x}+\varphi_{\mathrm{i}}\right)
$$

Where, 


$$
\begin{gathered}
Z_{i}=\frac{2}{F}\left|\hat{z}_{i}\right| \\
\varphi_{i}=\arg \left(\hat{\mathrm{z}}_{\mathrm{i}}\right) \quad \text { For } \mathrm{i}=0,1, \ldots, \mathrm{F} / 2 \\
\lambda_{i}=\frac{1}{f_{i}}=\frac{\mathrm{x}_{\mathrm{F}-1, \mathrm{al}}}{i}
\end{gathered}
$$

The Fourier coefficients in equation 3.12 can be interpreted as frequencies or wavelengths, which is referring to equation 3.16, with phase $\varphi_{i}$ and amplitude $Z_{i}$. Also from equation 3.16, it can be derived that the wavelength is dependent on the interval chosen, $\mathrm{x}_{\mathrm{F}-1, \mathrm{al}}$, which is dependent on the aliasing factor, $a$. Therefore, the aliasing factor has to be chosen such that it will not render the wavelength to be too large, thus loosing resolution in the calculations. Also, it must not be too small, going below the FFT data point limit of 4096 .

Through a plot of amplitude versus wavelength, it is possible to observe at which wavelength majority of the data is located. This can be used to identify the origin of the waviness. For example, the sewing thread that is used to keep the textile together occurs at regular intervals, which can be interpreted as wavelengths. From this plot, we can observe that majority of the data are located with a wavelength less than the stitch length, the distance between two rows of sewing thread. It can also be concluded from a plot of the distance travelled and the amplitude of the probe that at the short wavelength of the sewing thread, many steep amplitudes are produced. Therefore, the waviness caused by the sewing threads needs to be removed from the results to find the waviness of the fibres. In order to remove the influence of the sewing threads, a low pass filter is used. This filter removes all Fourier coefficients having a wavelength shorter than a particular value. With inverse transformation, equation 3.17, a smoothed curve is generated when used in equation 3.13.

$$
\mathrm{Z}_{\mathrm{i}, \mathrm{fil}}=\frac{1}{\mathrm{~F}} \sum_{\mathrm{k}=0}^{\mathrm{F}-1} \hat{z}_{k} \cdot \mathrm{e}^{\mathrm{j} \frac{2 \pi}{\mathrm{F}} \mathrm{ki}} \quad \text { For } \mathrm{i}=0,1, \ldots, \mathrm{F}-1 \text { and } \mathrm{j}^{2}=-1
$$

Where $\hat{z}_{k}$ are the filtered Fourier coefficients. 
The local angle of the fibre in the vertical $x z$-plane is:

$$
\alpha_{i, z x}=\arctan \left(\frac{z_{i+1, f i l}-z_{i, f i l}}{x_{i+1, n o r m}-x_{i, n o r m}}\right) \quad \text { For } i=0,1, \ldots, F-2
$$

The distribution of local fibre angles is used as a measure of the waviness of a textile. This distribution is found through "binning" or placing the angles into different angle ranges, with an interval of $0.1^{\circ}$. The count of angles that belong to each of these ranges is used to create a histogram, with frequency versus angle. The standard deviation of this frequency distribution is the SFO. Given that there are five in-fibre measurements and five SFO values, the average of these five values is taken as the SFO value for the entire textile. For additional in-fibre measurements, the SFO was found by the average of the standard deviation values. 


\section{CHAPTER 4: RELIABILITY MODEL FOR COMPOSITES}

\section{OUTLINE OF CHAPTER 4}

- The probability density functions (PDF) used for the model will be described.

- The background behind the "continuum damage model," used in the described reliability model, will be discussed.

- The graphical procedure to calculate $R$, reliability, will be discussed, as well as a proposed numerical procedure applied to the current situation, along with an example of calculating $R$ using the proposed procedure.

The proposed reliability model, developed by the author, is based on the "Strength Based Reliability and Interference Theory" described in Section 2.5 in Chapter 2. This model uses the fibre orientation found from Fibre Image Analysis, Result file 1, and the compression testing results from the laboratory as input data. Also, the formula used for calculating the compression strength of a ply based on the continuum damage model, in the Section on "prediction of failure," equation 2.23 , is also employed in this model.

In order to calculate the reliability, $R$, a tool is needed to facilitate tedious calculations. MATLAB software has been the chosen tool. The next Sections, 4.1 to 4.5 , describe how MATLAB's distribution fitting GUI and K-S (Kologorov-Smirnov) test has been used to calculate $R$. The data for the sensitivity curve, equation 2.23, and the fibre angle matrix found using Fibre Image Analysis, Section 3.4.1.2 in Chapter 3, are used "input data" in the model. However, before precise steps are given, some background on probability density functions (PDF), equation 2.23, and Rao’s (1992) Graphical Procedure are described.

\subsection{PROBABILITY DENSITY FUNCTION (PDF) FITTING}

MATLAB has a probability density function fitting tool, with a GUI interface. This interface can be called by typing, "dfittool." This tool has a number of built-in distributions that can be used to fit the data. The distributions that have been used to fit the data are: normal, lognormal, logistic, log-logistic, extreme value, and generalised extreme value distribution. These distributions were chosen from trial and error. They can have a skewed or symmetric "bell" shape. The formulas for each of the distributions are (MathWorks help, 1984-2011): 
Normal PDF:

$$
y=f(x \mid \mu, \sigma)=\frac{1}{\sigma \sqrt{2 \pi}} e^{\frac{-(x-\mu)^{2}}{2 \sigma^{2}}}
$$

Lognormal PDF:

$$
y=f(x \mid \mu, \sigma)=\frac{1}{x \sigma \sqrt{2 \pi}} e^{\frac{-(\ln x-\mu)^{2}}{2 \sigma^{2}}}
$$

Logistic PDF:

$$
y=f(x \mid \mu, \sigma)=\frac{e^{\frac{x-\mu}{\sigma}}}{\sigma\left(1+e^{\frac{x-\mu}{\sigma}}\right)^{2}}
$$

Log-logistic PDF (Meeker \& Escobar, 1998):

$$
y=f(x \mid \mu, \sigma)=\frac{1}{\sigma x} \frac{\exp \left(\frac{\log x-\mu}{\sigma}\right)}{\left[1+\exp \left(\frac{\log x-\mu}{\sigma}\right)\right]^{2}} \quad \text { For } \mathrm{x}>0
$$

Extreme Value PDF:

$$
y=f(x \mid \mu, \sigma)=\sigma^{-1} \exp \left(\frac{x-\mu}{\sigma}\right) \exp \left(-\exp \left(\frac{x-\mu}{\sigma}\right)\right)
$$

Generalised Extreme Value PDF:

For the shape parameter, $k \neq 0$

$$
y=f(x \mid k, \mu, \sigma)=\left(\frac{1}{\sigma}\right) \exp \left(-\left(1+k \frac{(x-\mu)}{\sigma}\right)^{-\frac{1}{k}}\right)\left(1+k \frac{(x-\mu)}{\sigma}\right)^{-1-\frac{1}{k}}
$$


Where

$1+k \frac{(x-\mu)}{\sigma}>0$

$k>0$ is Type II Case

$k<0$ is Type III Case

If $k=0$, then

$$
y=f(x \mid 0, \mu, \sigma)=\left(\frac{1}{\sigma}\right) \exp \left(-\exp \left(-\frac{(x-\mu)}{\sigma}\right)-\frac{(x-\mu)}{\sigma}\right)
$$

Where, $\mu$ is the location parameter and $\sigma$ is the scale parameter for all equations except equations 4.1 and 4.2 , in which case $\mu$ is the mean and $\sigma$ is the standard deviation.

Inverse Gaussian PDF:

$$
y=f(x \mid \mu, \lambda)=\sqrt{\frac{\lambda}{2 \pi x^{3}}} \exp \left\{-\frac{\lambda}{2 \mu^{2} x}(x-\mu)^{2}\right\}
$$

Where, $\mu$ is the mean, and $\lambda$ is the shape parameter.

\subsection{COMPRESSION STRENGTH OF A PLY BASED ON CONTINUUM DAMAGE MODEL}

The Continuum Damage Model by E.J. Barbero (1998) is based on the idea that fibres that have not buckled under compression loading share the load. The result is a curve that describes this failure process, with a maximum that indicates the maximum compression strength of the ply, the maximum compression loading that the ply can withstand. The compression strength of the composite can be found by equation 4.9. This equation is the same as equation 2.23, except that it is written differently.

$$
\sigma_{\mathrm{c}}=\max _{\alpha}\left[\sigma(\alpha) \int_{-\alpha}^{\alpha} \mathrm{f}\left(\alpha^{\prime}\right) \mathrm{d} \alpha^{\prime}\right]
$$


Inside the integral, the $\alpha$ values represent a folded distribution, since the CDF is only for half of the distribution $f$. This is the reason why there is an apostrophe beside $\alpha$.

$\sigma(\alpha)$ from equation 4.9 is found by:

$\sigma_{\mathrm{eq}}(\alpha, \gamma)=\frac{\tau_{\mathrm{u}}}{2(\gamma+\alpha)} \cdot \frac{(\sqrt{2}-1)\left(\mathrm{e}^{\sqrt{2} \mathrm{~g}}-\mathrm{e}^{2 \mathrm{~g}}\right)+(\sqrt{2}+1)\left(\mathrm{e}^{(2+\sqrt{2}) \mathrm{g}}-1\right)}{1+\mathrm{e}^{2 \mathrm{~g}}-\mathrm{e}^{\sqrt{2} \mathrm{~g}}+\mathrm{e}^{(2+\sqrt{2}) \mathrm{g}}}$

Where, $g=\gamma G_{L T} / \tau_{u}$

The plot of equation 4.10 (compression) over the same range of shear strain, $\gamma$, for different fibre misalignments, creates a stacked line graph as shown in Figure 63.

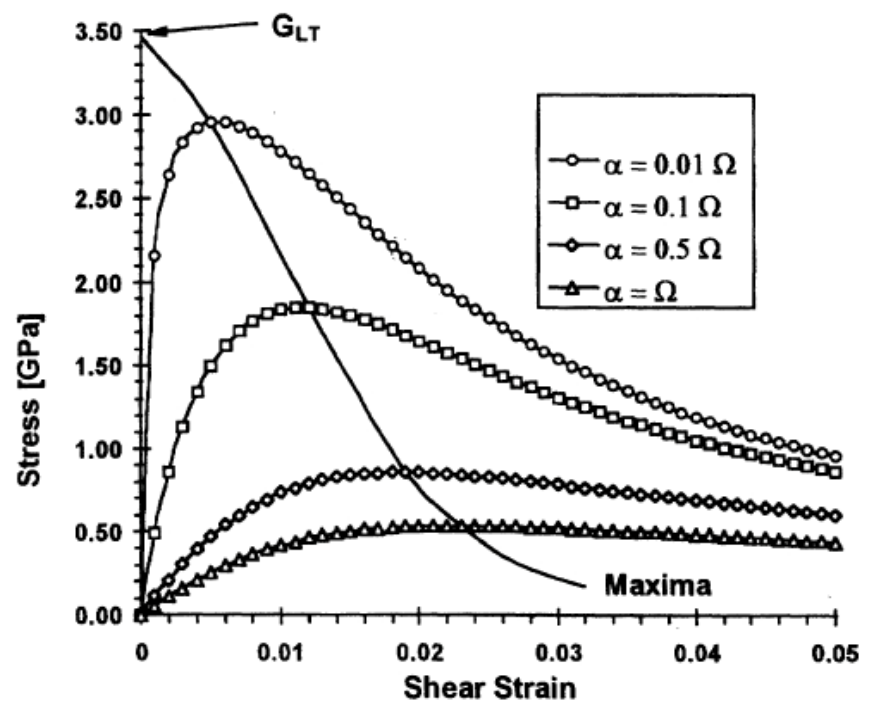

Figure 63: Equilibrium Stress for Various Fibre Misalignment Angles (a) (Barbero, 1998)

The maximum of each curve, which represents a different fibre misalignment, is plotted against the fibre misalignment to create the imperfection sensitivity curve, $\sigma(\alpha)$, as shown in Figure 64. 


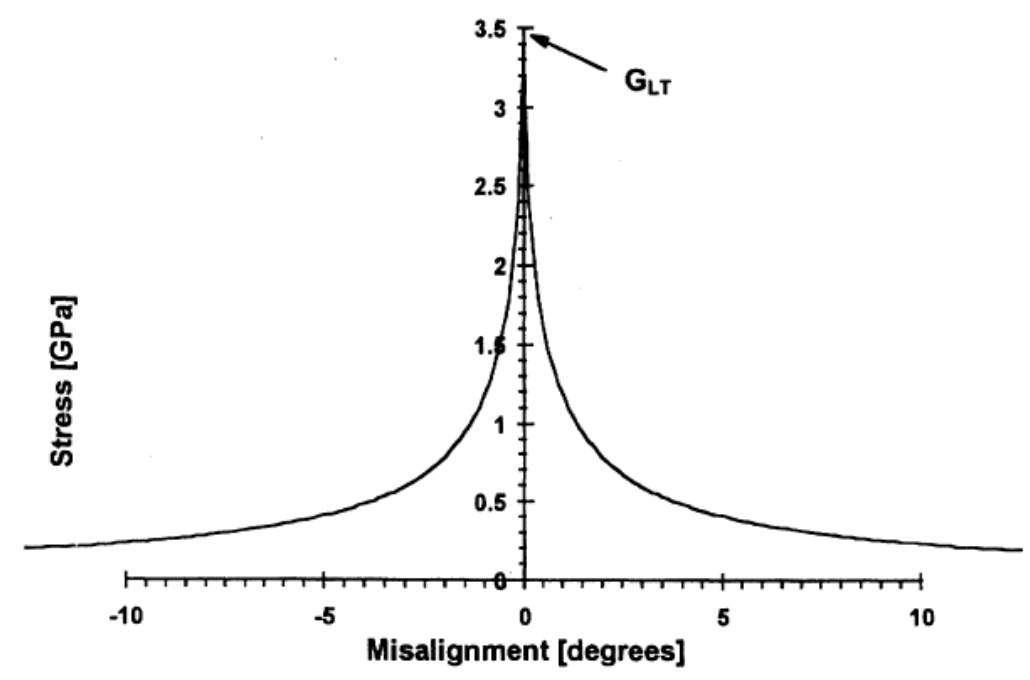

Figure 64: Imperfection Sensitivity (Barbero, 1998)

In Barbero's work, $\int_{-\alpha}^{\alpha} f\left(\alpha^{\prime}\right) \mathrm{d} \alpha^{\prime}$ from equation 4.10 is assumed to have a normal distribution, written as:

$$
f(\alpha)=\frac{1}{\Omega \sqrt{2 \pi}} e^{\frac{-\alpha^{2}}{2 \Omega^{2}}}
$$

Where, $\alpha$ is the misalignment angle (continuous random variable), and $\Omega$ is the standard deviation, both in $\left[^{\circ}\right]$.

\subsection{GRAPHICAL PROCEDURE}

The approach used here is based on S.S. Rao's "graphical procedure" (Rao, 1992) for finding the $R$-value with experimentally determined distributions of $S$ and $L$. Due to the complicated nature of the equations of $R$, equations 2.35 and 2.38, the use of either one of these equations to find $R$ is either through simulation, i.e. Monte Carlo simulation, where the parameter $l$ is set at a certain value prior to evaluating $R$, or by approximating the probability of failure (1-R) with first-order stochastic approximation (FORM), which uses first-order Taylor expansion (Wasserman, 2003).

The graphical method that S.S. Rao (Rao, 1992) used to find $R$ is as follows. It is important to emphasize that for steps 1 and 2, do not find the formulaic expression, just keep a note of the 
points for the curve. The $y$-axis should be probability, with 1 being the maximum number, and the $x$-axis should be stress/strength in the units of force per area.

1. Find $F_{S}(s)$. This is the cumulative distribution of the strength curve.

2. Find $F_{L}(l)$. This is the cumulative distribution of the load curve.

3. Define $X$ and $Y$ :

$$
\begin{aligned}
& X=\int_{0}^{l} f_{L}\left(l^{\prime}\right) d l^{\prime}=F_{L}(l) \\
& Y=\int_{l}^{\infty} f_{S}(s) d s=1-F_{S}(l)
\end{aligned}
$$

Find $X$ and $Y$ numerically for a corresponding stress/strength value. Plot $Y$ against $X$.

4. From equation $2.35, R=\int_{-\infty}^{\infty} f_{L}(l)\left[1-F_{S}(l)\right] d l$

Therefore,

$$
R=\int_{0}^{1} Y d X
$$

The range is chosen to be from 0 to 1 because $X$ goes from 0 to 1 . From equation 4.14 , it can be clearly seen that the area under the $Y$ versus $X$ graph is the reliability.

\subsection{PROPOSED NUMERICAL PROCEDURE, APPLIED TO CURRENT PROBLEM}

For the problem at hand, the strength, $S$, is chosen to be the compression strength results from the lab. These results represent the maximum compression loading that a sample of the material can take. After a laminate is made from the MWK textile, it can be cut into coupon samples for compression testing. Usually about six coupons can be cut from a laminate plate. Although sample size is small, these six results are directly fitted to find $f_{S}(s)$.

The load, $L$, is chosen to be the fibre misalignment angles. Variations in these angles would have an effect on ability for the fibres to bear load. The distribution of these angles can be 
found through analysing images of laminate samples using optical microscopy. However, in this study, the angle distribution is found from the "angle matrix" in the text file that results after analysing a scanned image with Fibre Image Analysis, as discussed in Section 3.4.1.2 in Chapter 3. This distribution is used to find the compression strength of the ply, corresponding to $f(\alpha)$ in equation 4.9. Still, the imperfection sensitivity curve, $\sigma(\alpha)$, needs to be found. This can be found through performing iterative laboratory tests set at a different $\alpha$ to find the distributions that satisfy equation 4.10, as shown in Figure 63. The maximum of each curve in Figure 63 is then graphed, this time with stress versus $\alpha$, to find the imperfection sensitivity curve, $\sigma(\alpha)$, as shown in Figure 64. Now, equation 2.23 can be numerically evaluated point by point to create a curve that represents the compression strength of the ply. However, in this study, the imperfection sensitivity curve was found using Patran, in the FMB analysis, discussed in Section 2.3 in Chapter 2. The curve that results from $\mathrm{f}(\alpha)$ and $\sigma(\alpha), \sigma_{\mathrm{c}}$, has the units of force per area for the $y$-axis and degrees of misalignment for the $x$-axis. Since $f_{L}(l)$ is a probability density function, with units of probability ( 0 to 1 ) for the $y$-axis and force per area for the $x$-axis, the $\sigma_{\mathrm{c}}$ curve needs to be fitted using only the force per area data to find $f_{L}(l)$.

Matlab has been extensively used to process the data collected for this research. Using Matlab's probability density function fitting tool, as mentioned in Section 4.1 in Chapter 4, the probability density function for load, $f_{L}(l)$, and for strength, $f_{S}(s)$, can be found. Using the same Matlab tool, specific cumulative function values for a specific force per area value can be found for the distribution that best describes the raw data. The distribution that best describes the raw data can be found by the $p$-value that is closest to 1 (Banks, Carson II, Nelson, \& Nicol, 2005). The Kolmogorov-Smirknov (K-S) test can be used to find the $p$-value (Banks, Carson II, Nelson, $\&$ Nicol, 2005). This test in Matlab also makes use of the cumulative function values.

Next, the equations 4.12 and 4.13 can be applied to find $X$ and $Y$. Aligning the $X$ and $Y$ values for a specific force per area value creates a point in the graph that represents $d R$. Collecting the $X$ and $Y$ values over a range of force per area values creates the entire graph. The area under the graph can now be approximated through the trapezoidal numerical integration function in Matlab, $\operatorname{trapz}(X, Y)$.

The precise steps are summarised as follows:

1. Find $f_{S}(s)$. Using the six compression testing samples: 
a. Use Matlab's probability density function fitting tool to fit the compression testing results to a PDF. Type "dfittool" in Matlab to open the GUI. Pick a variable name, enter equal, and paste in the 6 results. Save the variable as a ".mat" file for use in the dfittool GUI by typing "save" and the variable name. Click on "New Fit" to start fitting the data. Select the variable name as data source. Click on "Manage Fits" to manage the PDF's used to fit the data. Select the PDF's in Section 4.1 in Chapter 4 to fit the data.

b. Then, use K-S test to find the PDF with the highest $p$-value. To perform this test, the raw data's CDF values needs to be compared with the fitted distribution's $\mathrm{CDF}$ values. The CDF values of the fitted function can be found by clicking "Evaluate." Choose range that the CDF should be evaluated. This should correspond with your raw data's minimum and maximum values. The step interval should also be carefully chosen. Click on "Export to workspace" and choose an appropriate name. Then, the exported data needs to be split into $x$ (first column) and $y$ values (second column), where the $x$ represents the force per area data and the $y$ represents the cumulative probability. Here, the significance level for the data is set at 0.05 . Use $[h, p, k, c]=\operatorname{kstest}($ variable name in part $1 a,[x, y], 0.05$ ) to find the $p$-value.

c. Take the distribution with the highest $p$-value. Find the corresponding CDF values for this distribution, as shown in step $b$.

2. Find $f_{L}(l)$.

a. Use the FMB Patran model described in Section 2.3 in Chapter 2 to find $\sigma(\alpha)$, the imperfection sensitivity curve.

b. Use Fibre Image Analysis, as discussed in Section 3.4.1.2 in Chapter 3, to find the fibre misalignment angles. An Excel macro would need to be used to further process the fibre angle data: to assemble the data in a single column for easy processing using MATLAB, to remove empty cells (no fibre angle measurement) that represent masked domain regions or regions that contain defects, and to shift all angles below $90^{\circ}$ by $+180^{\circ}$ to ensure that the resulting angle distribution is "bell" shaped (not two halves of a "bell") and centres around $180^{\circ}$. Next, fit the angles using dfittool to find $f(\alpha)$. Evaluate $f(\alpha)$ at chosen $\alpha$ values and multiply it 
with the corresponding $\sigma(\alpha)$ value. Take the collection of resulting $\sigma_{\mathrm{c}}$ values and fit it with the dfittool, take the distribution with the highest $p$-value.

c. Find the CDF values for the resulting distribution.

3. Find $X$ and $Y$ according to equations 4.12 and 4.13. Insert the resulting $X$ and $Y$ values into Matlab. Use $\operatorname{trapz}(X, Y)$ to find the area under curve. The result is the $R$-value.

\subsection{AN EXAMPLE USING THE RELIABILITY MODEL}

The following hypothetical rounded data in Table 11 has been gathered for a piece of laminate:

\begin{tabular}{|l|l|}
\hline Coupon 1 & $500 \mathrm{MPa}$ \\
\hline Coupon 2 & $600 \mathrm{MPa}$ \\
\hline Coupon 3 & $610 \mathrm{MPa}$ \\
\hline Coupon 4 & $580 \mathrm{MPa}$ \\
\hline Coupon 5 & $590 \mathrm{MPa}$ \\
\hline Coupon 6 & $580 \mathrm{MPa}$ \\
\hline
\end{tabular}

Table 11: Hypothetical Compressing Testing Results

As Table 12 illustrates, the extreme value PDF has the highest $p$-value. Therefore, it should be taken as the best fit. This is $f_{S}(s)$, with $\mu=591.367$ and $\sigma=21.5514$. If the function is $\log$-logistic, then $\mu=\exp (a), \sigma=\exp (b)$, where $b$ and are the values found in Matlab. This is required to transform the found values back to the units of the raw data. Then, find the CDF values.

Fitting the data in Table 11 using the dfittool would result in the following $p$-values:

\begin{tabular}{|l|l|}
\hline Distribution & $p$-value \\
\hline Extreme Value & $\mathbf{0 . 6 4 5 8}$ \\
\hline Generalized Extreme Value & 0.3537 \\
\hline Inverse Gaussian & 0.2720 \\
\hline Log-logistic & 0.6162 \\
\hline Lognormal & 0.2847 \\
\hline Normal & 0.3131 \\
\hline Logistic & Not available \\
\hline
\end{tabular}

Table 12: $P$-values for Fitted PDF's for Hypothetical Compression Testing Results

Due to the large number of raw data values for finding $f(\alpha)$, it has not been included here. The procedure would be the same as to find $f_{S}(s)$ as previously explained. However, it is known that the it is a log-logistic distribution with $\mu=\operatorname{abs}(180-\exp (5.19447))=0.2725$ and 
$\sigma=\exp (0.00557111)=1.0056$. For the resulting values, the "exp" is needed to turn the values back into original units of measurement (MPa for $\mu$ in $f_{S}(s)$ and ${ }^{\circ}$ for $\mu$ here). In addition, $\mu$ for $\mathrm{f}(\alpha)$ requires 180 subtracted because $180^{\circ}$ means the same as $0^{\circ}$. Also, the negative sign is removed because $f(\alpha)$ is symmetric and would have the same probability value no matter the $\alpha$ is positive or negative. However, the log-logistic formula is sensitive to the data used in order for it to return meaningful results.

In Table 13, the distribution $\sigma_{\mathrm{c}}$ has been numerically found, see last column. The function $\mathrm{f}\left(\alpha^{\prime}\right)$, as previously found, has been evaluated at the $\alpha$ as shown in column 1 . Column 2 , which is the imperfection sensitivity curve, has been found to have the values in column 2 with the corresponding $\alpha$. It is important to note that Table 13 has some values omitted for clarity. The fibre misalignment angle, $\alpha$, had a finer resolution, a step size of 0.05 . As a result of this, there were interpolated values for $\sigma_{\mathrm{c}}$ and $\mathrm{f}\left(\alpha^{\prime}\right)$ was evaluated at those finer $\alpha$ values. This would enable the calculation of values in column $4, \int_{-\alpha}^{\alpha} \mathrm{f}\left(\alpha^{\prime}\right) \mathrm{d} \alpha^{\prime}$. In column 4 , the previous value is added to the current value. The current value is calculated by finding the result to the previous $\mathrm{f}\left(\alpha^{\prime}\right)$ plus current $\mathrm{f}\left(\alpha^{\prime}\right)$ divided by 2 and then multiplying this to the result of subtracting the previous $\alpha$ from the current $\alpha$ and divided by 2. Finally, the values in the last column, column 5 is found by multiplying the result from column 2 and 4 . Then, the result in column 5 is placed into Matlab's dfittool to find the PDF and the CDF, with the $p$-value results in Table 14.

\begin{tabular}{|l|l|l|l|l|}
\hline 1 & 2 & 3 & 4 & 5 \\
\hline \multicolumn{1}{|c|}{$\sigma(\alpha)$} & \multicolumn{1}{|c|}{$\mathrm{f}\left(\alpha^{\prime}\right)$} & $\int_{-\alpha}^{\alpha} \mathrm{f}\left(\alpha^{\prime}\right) \mathrm{d} \alpha^{\prime}$ & $\sigma(\alpha) \int_{-\alpha}^{\alpha} \mathrm{f}\left(\alpha^{\prime}\right) \mathrm{d} \alpha^{\prime}$ \\
\hline 0.1 & & & 0.36982 & 632.39007 \\
\hline 0.2 & 1710 & 1.70662 & 0.62961 & 1074.1067 \\
\hline 0.4 & 1700 & 0.99279 & 0.92350 & 1569.9545 \\
\hline 0.8 & 1500 & 0.55726 & 1.24597 & 1868.9539 \\
\hline 1.6 & 1100 & 0.15520 & 1.58606 & 1744.6619 \\
\hline 3.2 & 700 & 0.07666 & 1.92952 & 1350.6660 \\
\hline 4.5 & 600 & 0.05331 & 2.09510 & 1257.0570 \\
\hline 5 & 500 & 0.04755 & 2.14543 & 1072.7141 \\
\hline 6 & 400 & 0.03892 & 2.23138 & 892.55069 \\
\hline
\end{tabular}

Table 13: Finding $\sigma_{c}$ 


\begin{tabular}{|l|l|}
\hline Distribution & $p$-value \\
\hline Extreme Value & 0.3124 \\
\hline Generalized Extreme Value & $\mathbf{0 . 3 9 8 4}$ \\
\hline Inverse Gaussian & Not available \\
\hline Log-logistic & Not available \\
\hline Lognormal & Not available \\
\hline Normal & 0.0835 \\
\hline Logistic & 0.0998 \\
\hline
\end{tabular}

Table 14: P-values for Fitted PDF's for Results in "Column 5" of Table 13

The resulting generalised extreme value PDF has $\mu=902.182, \sigma=606.091$, and $k=-$ 0.542775 .

Table 15 illustrates some values for the $\mathrm{CDF}$ of $f_{L}(l)$ and $f_{S}(s)$ for the corresponding compression. Inserting the $X$ and $Y$ values into Matlab, under variables of the same name, the area of the graph that is created by $X$ and $Y$ can be found. To do this, enter the trapezoidal numerical integration function, $\operatorname{trapz}(X, Y)$. The resulting area of the graph, $R$, has been found to be 0.7974 . The calculated reliability, $R$, the probability of success, can have a value in the range [0-1], where a higher number would be represent a more ideal situation. The described process can be repeated for other MWK fabrics and the $R$ value will result which can facilitate the choosing of a textile to be used.

\begin{tabular}{|c|c|c|c|}
\hline $\begin{array}{l}\text { Compression } \\
{[\mathrm{MPa}]}\end{array}$ & $\begin{array}{l}\quad X=\int_{0}^{l} f_{L}\left(l^{\prime}\right) d l^{\prime}=F_{L}(l) \\
\text { CDF for Compression } \\
\text { Testing Results }\end{array}$ & $\begin{array}{l}\quad F_{S}(l) \\
\text { CDF for } \\
\text { fitted } \\
\text { "Column 5" }\end{array}$ & $\begin{array}{l}Y=\int_{l}^{\infty} f_{S}(s) d s=1-F_{S}(l) \\
\text { 1-CDF for fitted "Column } 5 \text { " } \\
\text { of Table } 13\end{array}$ \\
\hline$\overline{0}$ & 0 & 0.0509 & 0.9491 \\
\hline 333 & 0 & 0.1181 & 0.8819 \\
\hline 666 & 1 & 0.2407 & 0.7593 \\
\hline 999 & 1 & 0.4291 & 0.5709 \\
\hline 1332 & 1 & 0.6647 & 0.3353 \\
\hline 1665 & 1 & 0.8866 & 0.1134 \\
\hline 1896 & 1 & 0.983 & 0.017 \\
\hline
\end{tabular}

Table 15: $X$ and $Y$ 


\section{CHAPTER 5: RESULTS AND CONCLUSIONS}

\section{OUTLINE OF CHAPTER 5:}

- The results will be given first, and then a discussion of the results, discussion of possible future work, and finally the conclusion is given.

\subsection{RESULTS}

As mentioned in the previous Chapter, laboratory tests were performed using laminates. These results were compared to the predicted compression strength found using the combined FMB-PMB and Unit Cell model described in Chapter 3. These laboratory tests were performed on laminates made with the Vacuum Infusion technique, described in Chapter 1, using carbon fibre textiles infused with epoxy resin. These finished laminates were then tested using ultrasonic testing (C-Scan) for areas that contain defect and then the appropriate "cutting plan" is developed, where six coupons are cut. These coupons then undergo compression testing, as mentioned in Section 2.2.2.2 in Chapter 2, and the maximum compression that the coupon can withstand before failure is recorded. The average of the six maximum compression values that each coupon can withstand is taken as the compression strength for a material.

Also, the PMB model described in Section 2.3 in Chapter 2, Figure 29, involves a "unit cell" of a 0/45/90/135/0/135/90/45/0 layup of textiles. For the textiles analysed, the layup is $\left[(0 / 90)_{4}\right]_{s}$, as illustrated in Figure 42 in Section 3.3 in Chapter 3. Since the compression strength results are found from the middle $0^{\circ}$ layer in the PMB model, a "unit cell" needed to be created to represent the $0 / 90$ textile. Therefore, in the PMB "unit cell" was modified to 0/90/90/90/0/90/90/90/0. Here, the three consecutive $90^{\circ}$ layers together have the same height as the centre $0^{\circ}$ layer, and the two $0^{\circ}$ layers at the end together also have the same height as the centre $0^{\circ}$ layer. This means that $50 \%$ of the "unit cell" is comprised of $0^{\circ}$ layers and $50 \%$ is comprised of $90^{\circ}$ layers. The height, in millimetres, is calculated from the areal weight of the textile, finding $\mathrm{t}$ in equation 3.2 with $m_{a}=0, n=1$, and not multiplying by $100 \%$. For the PMB model, if the Up Side is being simulated, with the centre being $0^{\circ}$ in a layup of 0/90/90/90/0/90/90/90/0, then the elastic modulus corresponds with the height. For example, the elastic modulus property of $0^{\circ}$ layers corresponds to a height for $0^{\circ}$ layers, and the same goes with $90^{\circ}$ layers. If the Down Side is being simulated, then this is reversed. The centre is $90^{\circ}$ in a 
layup of $90 / 0 / 0 / 0 / 90 / 0 / 0 / 0 / 90$, the elastic modulus is reversed with the height. Here, the height for the $90^{\circ}$ layer corresponds with the elastic modulus property for the $0^{\circ}$ layer. This reversal is necessary because the actual compression tests in the laboratory are always tested in the direction of the fibre. So, for tests on the Down Side, the $90^{\circ}$ layer, the applied compressive force will be $0^{\circ}$ from the fibre and would therefore have the properties of a $0^{\circ}$ layer. See Figure 65 . The black lines here represent the orientation of the fibres.

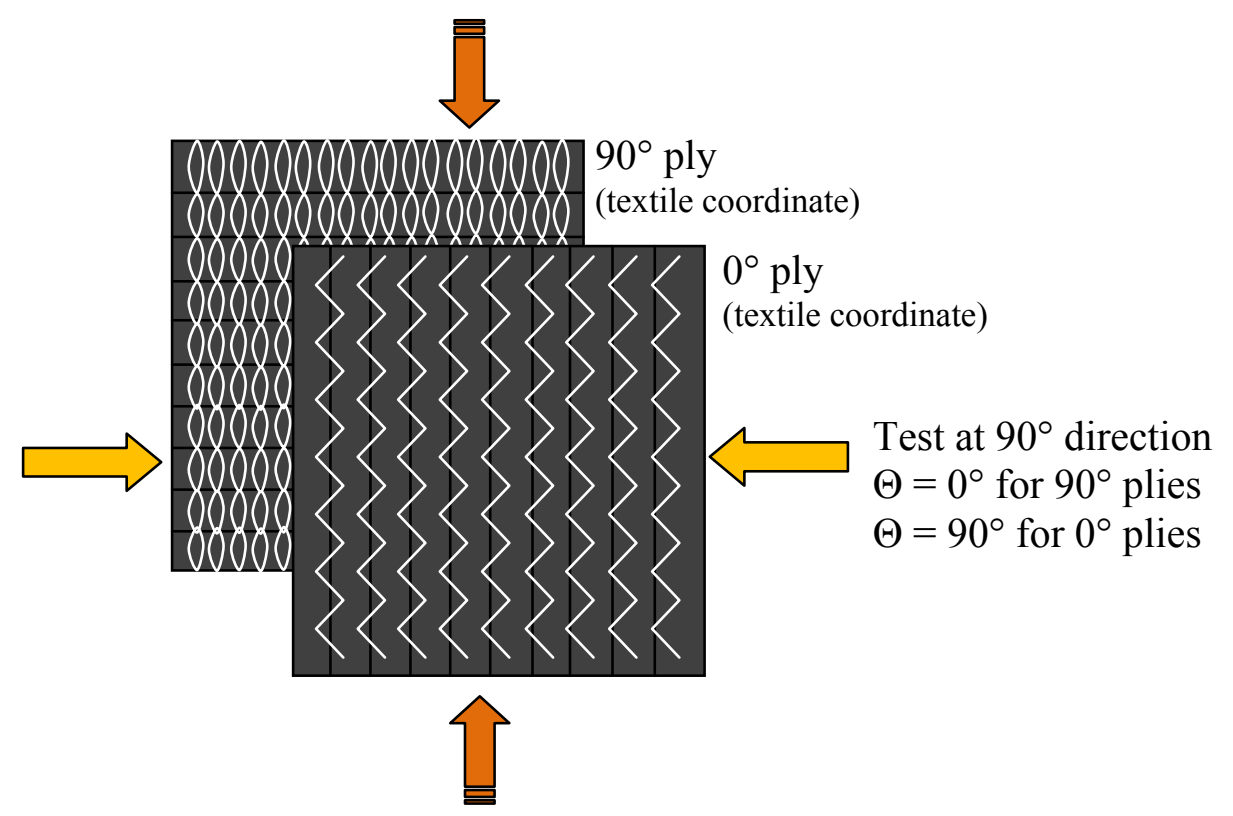

Test at $0^{\circ}$ direction

$\Theta=0^{\circ}$ for $0^{\circ}$ plies

$\Theta=90^{\circ}$ for $90^{\circ}$ plies

Figure 65: 0/90 laminates, Interpreting Angle $\Theta$

Since some of the 0/90 textiles were sheared, some adjustments also need to be made in the PMB model due to shearing. For the laminates made with sheared textiles, they were tested in the $0^{\circ}$ and the $75^{\circ}$ direction, as opposed to the $0^{\circ}$ and the $90^{\circ}$ direction, as previously mentioned. See Figure 66. However, $75^{\circ}$ to $90^{\circ}$ was assumed to have a negligible difference on the elastic modulus property. Therefore, the PMB model was not modified to account for this difference. However, this difference is taken into account during "normalisation" calculations. Normalisation is necessary because the results from the PMB model were only for the middle layer, an UD ply, and there were eight layers in the 0/90 layup. After the compression strength 
predictions have been obtained from the FMB-PMB Unit Cell model, the results need to be "normalised" to the thickness of the entire laminate, according to Classical Lamination Theory (CLT). The steps for this are illustrated in Appendix C.

In addition to 0/90 laminates and sheared laminates, 0/75 laminates, one 135/0/45/90/45/0/135 laminate was analysed. The PMB model was accordingly adjusted to account for the correct height and elastic modulus of each layer, according to the areal weight and the test direction, as shown in Figure 67. However, since it used a different type of carbon fibre than the other textiles, and it was the only of its kind that was analysed, a "correction factor," could not be calculated. Therefore, the final results for this type of textile have not been pursued to the end.

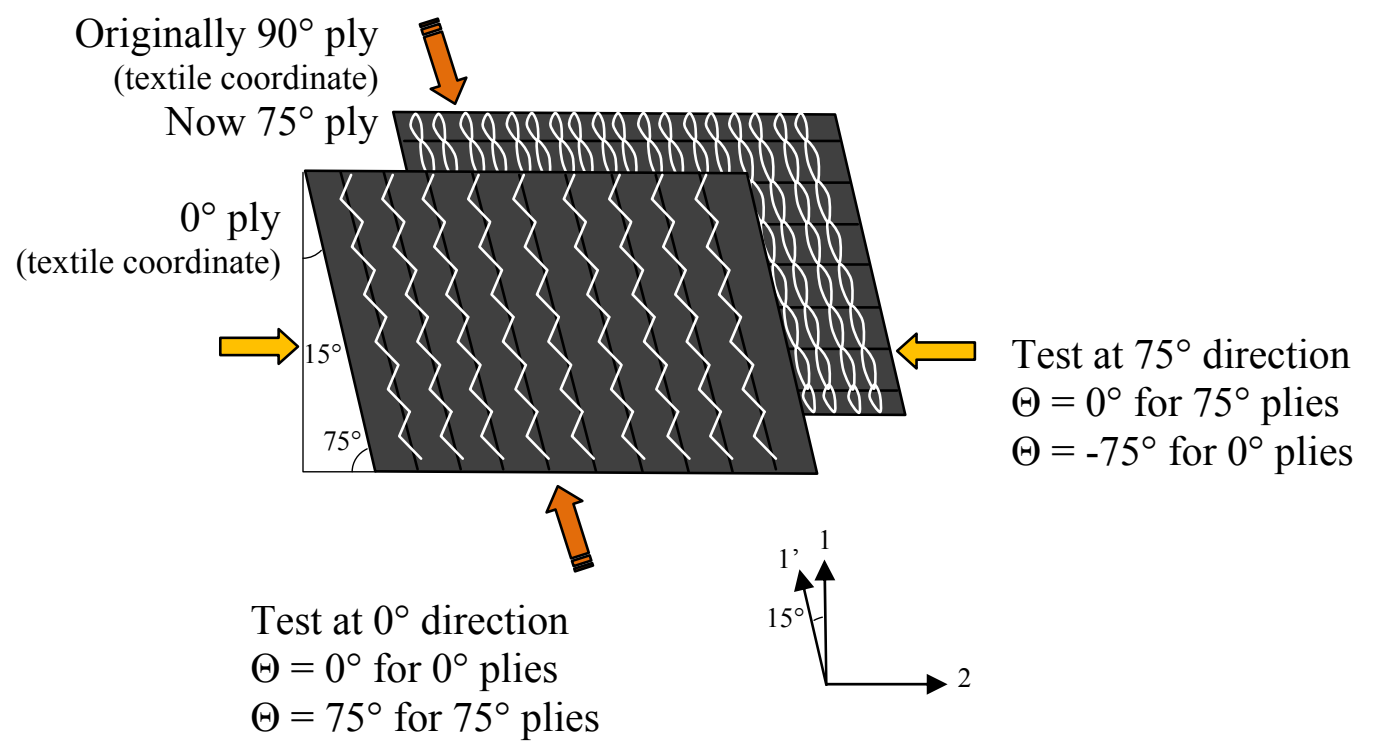

Figure 66: 0/75 laminates, Interpreting Angle $\Theta$

The "correction factor" was found using the Least Squares Method. Here, the "correction factor" is the number, when multiplied to the normalised predicted compression strength, is the minimum of the sum of squares. The sum of squares is the sum of the square of the difference between the actual compression strength value and the corrected and normalised predicted compression strength value. This correction value can be found using MS Excel's solver function. This was done to find the relative differences between the predictions and the actual results. 


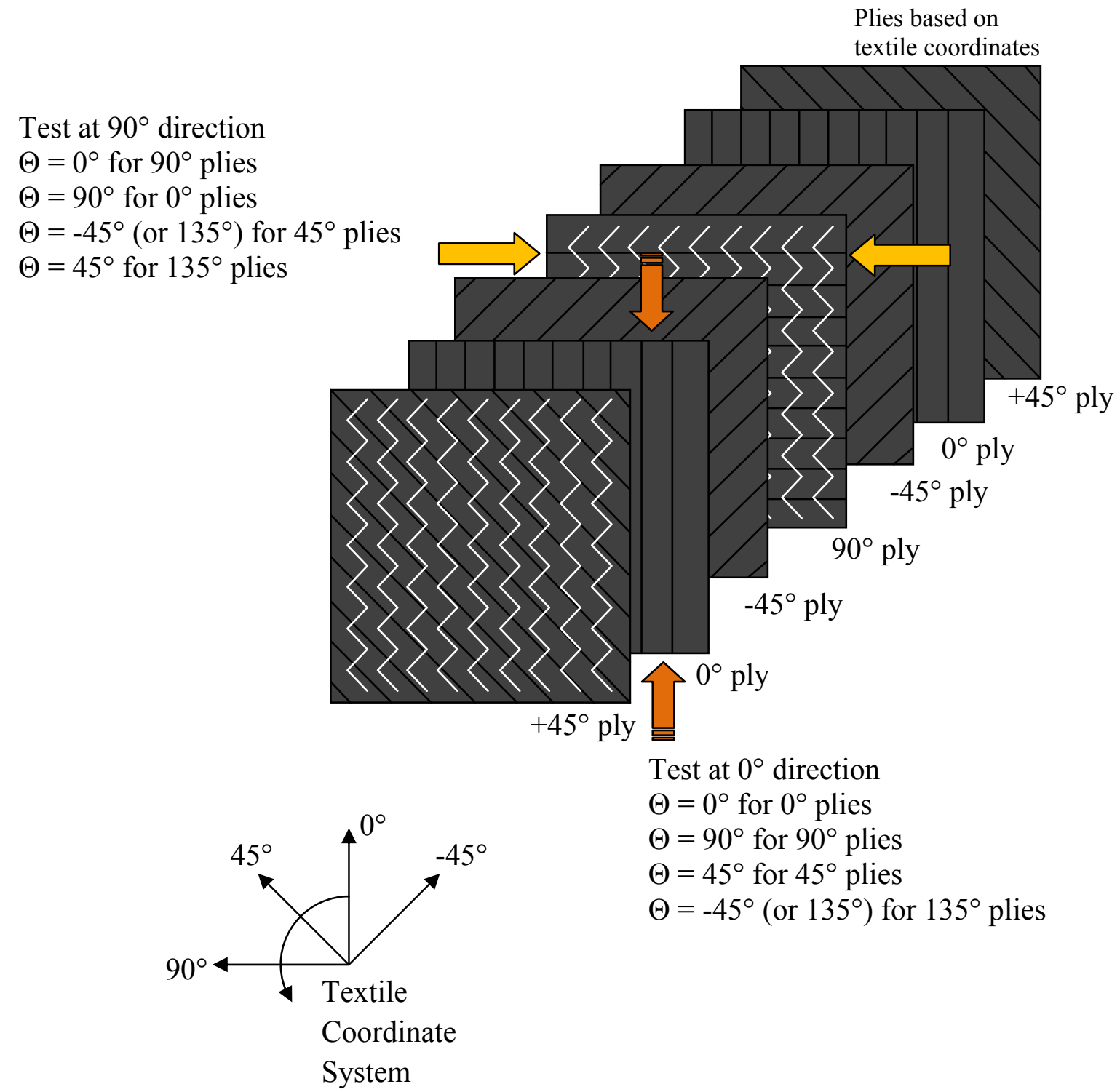

Figure 67: 135/0/45/90/45/0/135 laminates, Interpreting Angle $\Theta$

The following pages give the results of the study, in tables and figures. It is important to note that the SFI values for 2400 dpi images are not given in the following tables because given the small domain size analysed (50 x 50 pixels) using Fibre Image Analysis, the SFI value was very large. When encountered with large SFI values, values greater than about three, the FMB model would always return the same result. Equation 2.23 would always have a result of 491.371456457143 MPa. Therefore, only the SFI values from 720 dpi images were used. These scanned images were also analysed with a domain size of 50 x 50 pixels using Fibre Image Analysis. 


\begin{tabular}{llll}
\hline & $\begin{array}{l}\text { analySIS } \\
{[\mathbf{M P a}]}\end{array}$ & $\begin{array}{l}\text { Fibre Image Analysis } \\
{[\mathbf{M P a}]}\end{array}$ & $\begin{array}{l}\text { Actual Results, Average } \\
{[\mathrm{MPa}]}\end{array}$ \\
\hline K1-1 Sh (DS) & 521.58 & 442.34 & 532.50 \\
K1-2 (DS) & 539.09 & 432.35 & 529.72 \\
\hline $\mathbf{1 4 - 2}$ (DS) & 615.45 & 556.63 & 597.92 \\
$\mathbf{1 4 - 2}$ (US) & 646.75 & 674.74 & 507.46 \\
$\mathbf{1 1 - 1}$ Sh (DS) & 709.44 & $*$ & 596.52 \\
$\mathbf{1 1 - 2}$ (DS) & 693.79 & 565.35 & 562.31 \\
$\mathbf{1 1 - 2}$ (US) & 724.76 & 670.12 & 647.31 \\
$\mathbf{4 0 0 - 1}$ Sh (DS) & 516.55 & 450.16 & 509.03 \\
$\mathbf{4 0 0 - 2}$ (DS) & 498.23 & 440.75 & 533.17 \\
$\mathbf{4 0 1}$ Sh (DS) & 551.27 & 471.63 & 560.42 \\
401-2 (DS) & 470.27 & 430.62 & 731.21 \\
397 (DS) & 566.38 & 434.53 & 603.41 \\
397 (US) & 628.56 & 658.14 & 549.19 \\
\hline censored data & & &
\end{tabular}

Table 16: (Normalised and Corrected, with Correction Factor $=\mathbf{2 . 6 4 1 3 0 4 5 6 4 8 0 4 9 )}$ Predicted Compression Results from analySIS and Fibre Image Analysis, Compared to Laboratory Results

The result for 11-1 Sh (DS) using Fibre Image Analysis has been censored because there has been an error with finding the sensitivity curve from $\tilde{\sigma}_{11}^{c u, F M B}$, equation 2.29 , after $\tilde{\sigma}_{11}^{c u, F M B}$ has been used to create the sensitivity curve for FMB PMB on basis of SFO, as described in Section 3.1 in Chapter 3. The predicted compression strength value was very high for this textile as a result of this error.

\begin{tabular}{ll}
\hline & R value (Ch. 4) \\
\hline K1-1 Sh (DS) & 0.00011973 \\
K1-2 (DS) & 0.1283 \\
$\mathbf{1 4 - 2}$ (DS) & 0.000008465 \\
$\mathbf{1 4 - 2}$ (US) & 0.7841 \\
$\mathbf{1 1 - 1}$ Sh (DS) & 0.7826 \\
$\mathbf{1 1 - 2}$ (DS) & 0.6893 \\
$\mathbf{1 1 - 2}$ (US) & 0.738 \\
$\mathbf{4 0 0 - 1}$ Sh (DS) & 0.8143 \\
$\mathbf{4 0 0 - 2}$ (DS) & 0.8024 \\
$\mathbf{4 0 1}$ Sh (DS) & 0.1599 \\
$\mathbf{4 0 1 - 2}$ (DS) & 0.1034 \\
$\mathbf{3 9 7}$ (DS) & 0.2894 \\
$\mathbf{3 9 7}$ (US) & 0.332 \\
\hline
\end{tabular}


The percent difference is calculated:

Percent Difference $=\frac{\mid \text { Actual Value }- \text { Experimental Value } \mid}{\text { Actual Value }} \cdot 100 \%$

\begin{tabular}{llc}
\hline & \multicolumn{2}{l}{ Percent Difference between Actual Results } \\
\hline K1-1 Sh (DS) & 2.05 & 16.93 \\
K1-2 (DS) & 1.77 & 18.38 \\
$\mathbf{1 4 - 2}$ (DS) & 2.93 & 6.91 \\
$\mathbf{1 4 - 2}$ (US) & 27.45 & 32.96 \\
$\mathbf{1 1 - 1}$ Sh (DS) & 18.93 & $*$ \\
$\mathbf{1 1 - 2}$ (DS) & 23.38 & 0.54 \\
$\mathbf{1 1 - 2}$ (US) & 11.97 & 3.52 \\
$\mathbf{4 0 0 - 1}$ Sh (DS) & 1.48 & 11.56 \\
$\mathbf{4 0 0 - 2}$ (DS) & 6.55 & 17.33 \\
$\mathbf{4 0 1}$ Sh (DS) & 1.63 & 15.84 \\
$\mathbf{4 0 1 - 2}$ (DS) & 35.69 & 41.11 \\
$\mathbf{3 9 7}$ (DS) & 6.14 & 27.99 \\
$\mathbf{3 9 7}$ (US) & 14.45 & 19.84 \\
& $\mathbf{m i n}$ & $\mathbf{1 . 4 8}$ \\
\hline *consored data & $\mathbf{3 5 . 6 9}$ & $\mathbf{4 1 . 1 1}$ \\
\hline
\end{tabular}

Table 18: Percent Difference between Actual Results 


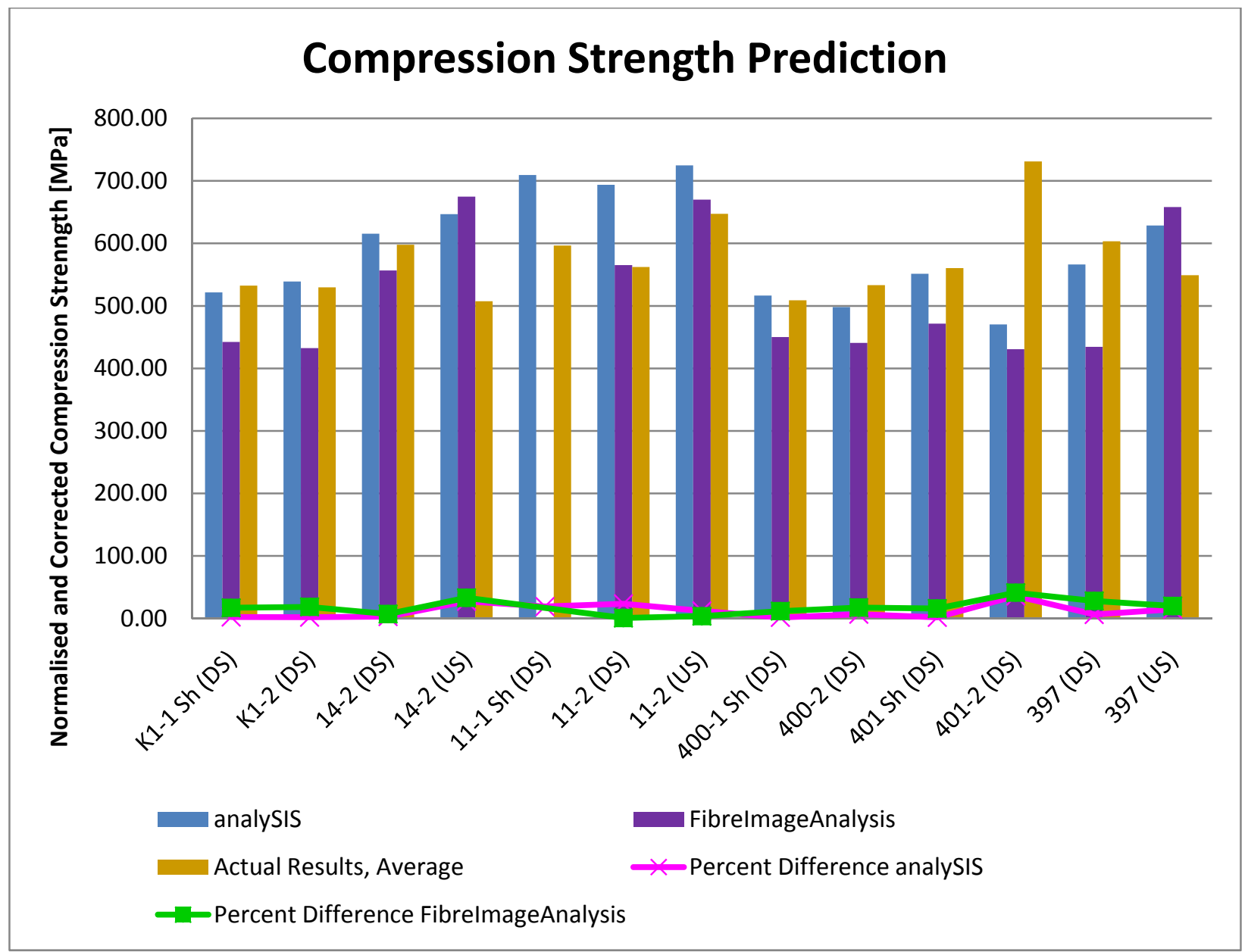

Figure 68: (Normalised and Corrected) Predicted Compression Strength vs. Textile, Plot of Table 16 and Table 18 
Anova: Two-Factor Without Replication

\begin{tabular}{lllll}
\hline SUMMARY & Count & Sum & Average & Variance \\
\hline K1-1 Sh (DS) & 3 & 1496.418 & 498.806 & 2421.466 \\
K1-2 (DS) & 3 & 1501.162 & 500.3873 & 3493.438 \\
$14-2$ (DS) & 3 & 1769.989 & 589.9963 & 911.957 \\
$14-2$ (US) & 3 & 1828.953 & 609.6509 & 8028.092 \\
$11-2$ (DS) & 3 & 1821.448 & 607.1492 & 5631.663 \\
$11-2$ (US) & 3 & 2042.187 & 680.7291 & 1584.417 \\
$400-1$ Sh (DS) & 3 & 1475.731 & 491.9102 & 1321.478 \\
$400-2$ (DS) & 3 & 1472.155 & 490.7182 & 2177.479 \\
401 Sh (DS) & 3 & 1583.319 & 527.7729 & 2385.342 \\
$401-2$ (DS) & 3 & 1632.104 & 544.0346 & 26668.83 \\
397 (DS) & 3 & 1604.311 & 534.7704 & 7879.657 \\
397 (US) & 3 & 1835.888 & 611.9626 & 3174.162 \\
& & & & \\
analySIS & 12 & 6972.657 & 581.0547 & 6437.385 \\
$\begin{array}{l}\text { FibreImageAnalysis } \\
\text { Actual Results, }\end{array}$ & 12 & 6227.361 & 518.9468 & 10132.2 \\
Average & & & & \\
\hline
\end{tabular}

\begin{tabular}{|c|c|c|c|c|c|c|}
\hline Source of Variation & $S S$ & $d f$ & $M S$ & $F$ & $P$-value & $F$ crit \\
\hline Rows & 124326.9 & 11 & 11302.45 & 2.382877 & 0.039949 & 2.258518 \\
\hline Columns & 27005.71 & 2 & 13502.86 & 2.846786 & 0.079521 & 3.443357 \\
\hline Error & 104350.2 & 22 & 4743.193 & & & \\
\hline Total & 255682.9 & 35 & & & & \\
\hline Total & 297853.4 & 38 & & & & \\
\hline
\end{tabular}

A detailed discussion of the concepts behind ANOVA (Analysis of Variance) can be obtained from Montgomery (2005) and ANOVA can be easily performed with MS Excel, under "Data Analysis Tools."

It can be seen from Table 19 that the $p$-value for rows is below 0.05 and for columns is over 0.05 , where 0.05 is the value of alpha used. Therefore, we reject the null hypothesis for the rows (textiles) and fail to reject the null hypothesis for the columns (analySIS and Fibre Image Analysis). The textiles are not the same $(p$-value $=\mathbf{0 . 0 3 9 9 4 9})$ and analySIS and Fibre Image Analysis give practically the same results $(p$-value $=\mathbf{0 . 0 7 9 5 2 1})$. 


\begin{tabular}{|c|c|c|c|c|c|c|c|c|c|}
\hline \multicolumn{7}{|c|}{ Pearson Product-Moment CORRELATION Coefficient, $r$ : } & \multicolumn{2}{|c|}{$\begin{array}{l}\text { Percent difference } \\
\text { between Actual } \\
\text { Results } \\
\end{array}$} & \multirow[b]{2}{*}{$\begin{array}{l}R \\
\text { value } \\
\text { (Ch. 5) }\end{array}$} \\
\hline & $S F I\left[^{\circ}\right]$ & $\begin{array}{l}\text { Comp. } \\
\text { analySIS } \\
{[M P a]}\end{array}$ & $\begin{array}{l}S F I \\
{\left[{ }^{\circ}\right]}\end{array}$ & $\begin{array}{l}\text { Comp. } \\
\text { Fibre } \\
\text { Image } \\
\text { Analysis } \\
{[\mathrm{MPa}]}\end{array}$ & $\begin{array}{l}\text { Compression } \\
\text { Actual } \\
\text { Results, } \\
\text { Average } \\
\text { [MPa] }\end{array}$ & $\begin{array}{l}S F O \\
{\left[{ }^{\circ}\right]}\end{array}$ & analySIS & $\begin{array}{l}\text { Fibre } \\
\text { Image } \\
\text { Analysis }\end{array}$ & \\
\hline $\begin{array}{l}\text { SFI } \\
\text { analySIS } \\
\left.{ }^{\circ}\right]\end{array}$ & $\begin{array}{l}1 \\
-0.79\end{array}$ & 1.00 & & & & & & & \\
\hline & 0.33 & -0.59 & 1.00 & & & & & & \\
\hline $\begin{array}{l}\text { Fibre } \\
\text { Image } \\
\text { Analysis } \\
\text { [MPa] } \\
\text { Actual } \\
\text { Results, } \\
\text { Average } \\
\text { [MPa] }\end{array}$ & -0.09 & 0.09 & -0.10 & -0.12 & 1.00 & & & & \\
\hline SFO [ [ & -0.59 & 0.31 & 0.32 & 0.21 & -0.72 & 1.00 & & & \\
\hline analySIS & -0.38 & 0.44 & -0.53 & 0.08 & 0.36 & $\begin{array}{r}- \\
0.07\end{array}$ & 1.00 & & \\
\hline $\begin{array}{l}\text { Fibre } \\
\text { Image } \\
\text { Analysis }\end{array}$ & 0.09 & -0.24 & -0.14 & 0.08 & 0.25 & 0.28 & 0.54 & 1.00 & \\
\hline $\begin{array}{l}\text { R value } \\
\text { (Ch. 5) }\end{array}$ & -0.13 & 0.44 & -0.27 & -0.06 & -0.26 & 0.11 & 0.32 & -0.05 & 1.00 \\
\hline
\end{tabular}

Table 20: Correlation between R value, SFI, SFO, and Compression Strength Values, Data from Table 16, Table 17, Table 18, Table 21, and Table 22

Correlation calculations can be easily performed with MS Excel, under "Data Analysis Tools." Correlation values range between -1 to +1 , where a 0 value means that the two data do not vary with each other. A negative value means that when one variable increases, the other decreases and vice versa. A positive value means that when one variable increases, the other increases, and when one decreases, the other decreases.

According to Table 20, a fairly strong positive correlation can be observed between the $R$ value and the compression strength prediction from analySIS (0.44), and the percent difference between analySIS and the laboratory compression values (0.32). A small positive correlation between the $R$ value (Ch. 5) and the SFO can be seen (0.11). 
A negative correlation can be observed between the $R$ value (Ch. 5) and the SFI found with analySIS (-0.13), the SFI found with Fibre Image Analysis (-0.27), the compression results from Fibre Image Analysis (-0.06), the compression results from the laboratory $(-0.26)$, and the percent difference between Fibre Image Analysis and the laboratory compression values (-0.05).

\begin{tabular}{l|ll|ll}
\hline & SFI $^{\circ}{ }^{\circ}$ & analySIS [MPa] & SFI $\left.^{\circ}{ }^{\circ}\right]$ & Fibre Image Analysis [MPa] \\
\hline K1-1 Sh (DS) & 1.668 & 521.58 & 6.266 & 442.34 \\
K1-2 (DS) & 1.509 & 539.09 & 4.711 & 432.35 \\
$\mathbf{1 4 - 2}$ (DS) & 1.504 & 615.45 & 2.008 & 556.63 \\
$\mathbf{1 4 - 2}$ (US) & 1.357 & 646.75 & 2.792 & 674.74 \\
$\mathbf{1 1 - 1}$ Sh (DS) & 1.336 & 709.44 & 2.52 & $*$ \\
$\mathbf{1 1 - 2}$ (DS) & 1.542 & 693.79 & 2.304 & 565.35 \\
$\mathbf{1 1 - 2}$ (US) & 1.520 & 724.76 & 3.083 & 670.12 \\
$\mathbf{4 0 0 - 1}$ Sh (DS) & 1.951 & 516.55 & 5.589 & 450.16 \\
$\mathbf{4 0 0 - 2}$ (DS) & 2.098 & 498.23 & 3.111 & 440.75 \\
$\mathbf{4 0 1}$ Sh (DS) & 1.921 & 551.27 & 4.571 & 471.63 \\
$\mathbf{4 0 1 - 2}$ (DS) & 2.105 & 470.27 & 3.293 & 430.62 \\
$\mathbf{3 9 7}$ (DS) & 1.945 & 566.38 & 3.196 & 434.53 \\
397 (US) & 1.424 & 628.56 & 2.869 & 658.14 \\
\hline
\end{tabular}

*censored data

Table 21: SFI values for analySIS and Fibre Image Analysis

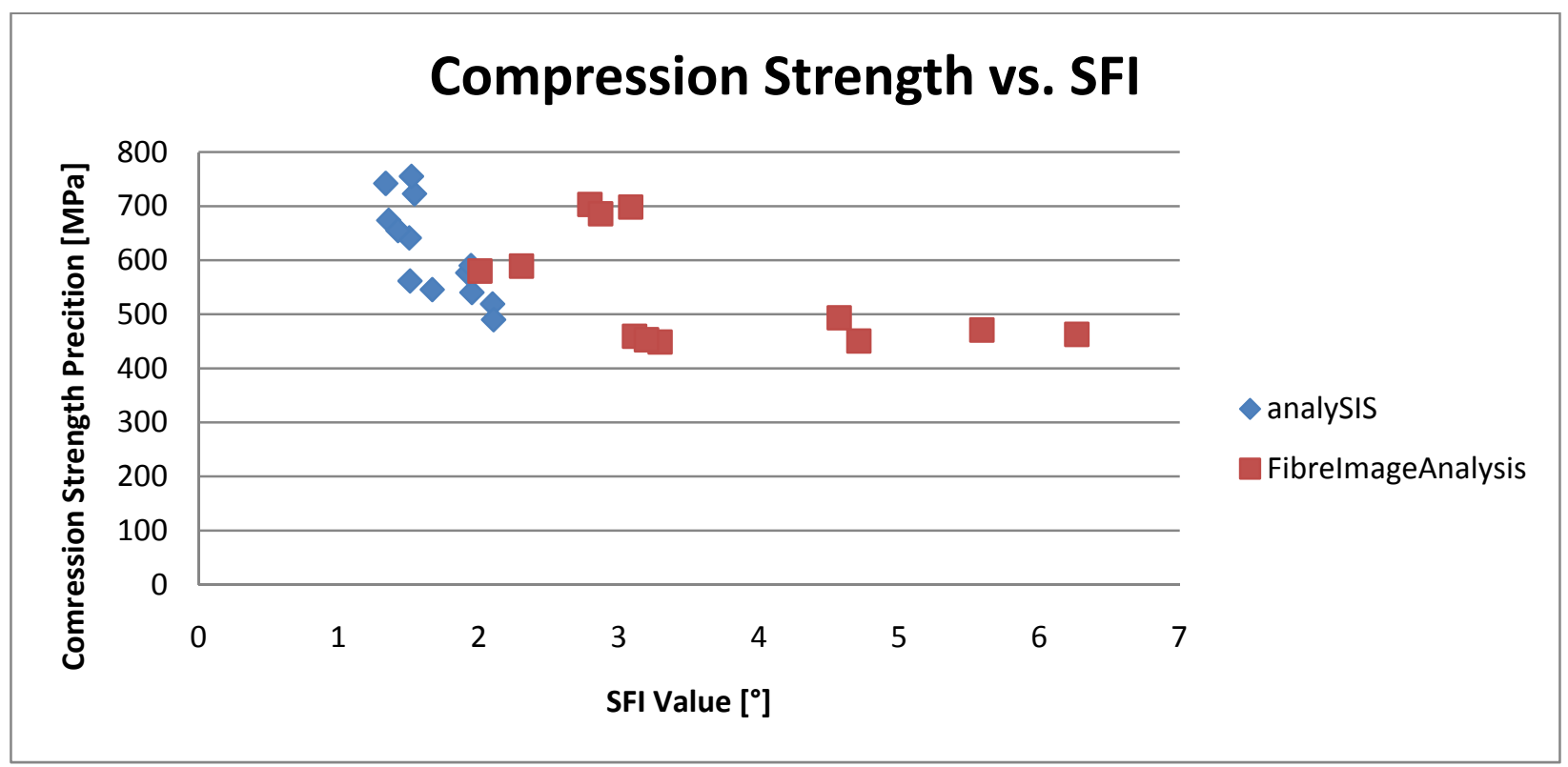

Figure 69: Plot of Table 21, Predicted Compression Strength versus SFI 


\begin{tabular}{l|l|ll}
\hline & SFO [ $\left.{ }^{\circ}\right]$ & analySIS [MPa] & Fibre Image Analysis [MPa] \\
\hline K1-1 Sh (DS) & 2.500 & 521.58 & 442.34 \\
K1-2 (DS) & 2.226 & 539.09 & 432.35 \\
$\mathbf{1 4 - 2}$ (DS) & 1.945 & 615.45 & 556.63 \\
$\mathbf{1 4 - 2}$ (US) & 2.499 & 646.75 & 674.74 \\
$\mathbf{1 1 - 1}$ Sh (DS) & 2.077 & 709.44 & $*$ \\
$\mathbf{1 1 - 2}$ (DS) & 2.051 & 693.79 & 565.35 \\
$\mathbf{1 1 - 2}$ (US) & 2.024 & 724.76 & 670.12 \\
$\mathbf{4 0 0 - 1}$ Sh (DS) & 2.253 & 516.55 & 450.16 \\
$\mathbf{4 0 0 - 2}$ (DS) & 1.664 & 498.23 & 440.75 \\
$\mathbf{4 0 1}$ Sh (DS) & 2.409 & 551.27 & 471.63 \\
$\mathbf{4 0 1 - 2}$ (DS) & 1.027 & 470.27 & 430.62 \\
$\mathbf{3 9 7}$ (DS) & 1.149 & 566.38 & 434.53 \\
397 (US) & 2.492 & 628.56 & 658.14 \\
\hline
\end{tabular}

*censored data

Table 22: SFO versus Compression Strength

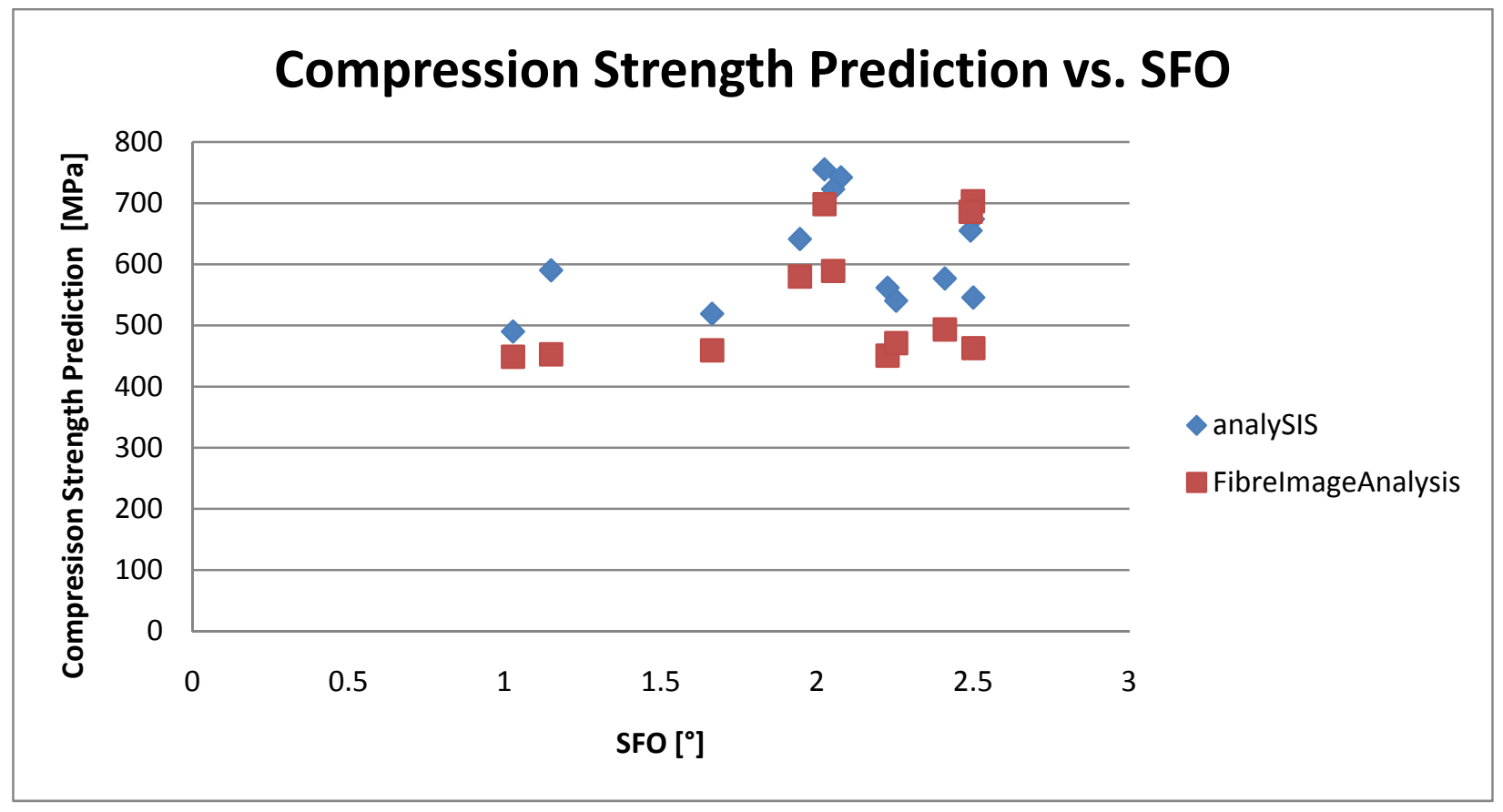

Figure 70: Plot of Table 22, Predicted Compression Strength versus SFO 


\begin{tabular}{llll}
\hline & width, b [mm] & length, $\mathbf{l}[\mathbf{m m}]$ & analySIS [MPa] \\
\hline K1-1 Sh (DS) & 0.1975 & 3.8135 & 521.58 \\
K1-2 (DS) & 0.2131 & 3.5047 & 539.09 \\
14-2 (DS) & 0.3041 & 7.2983 & 615.45 \\
14-2 (US) & 0.2336 & 4.3441 & 646.75 \\
11-1 Sh (DS) & 0.1762 & 5.7751 & 709.44 \\
11-2 (DS) & 0.1647 & 7.1579 & 693.79 \\
11-2 (US) & 0.1659 & 5.0660 & 724.76 \\
400-1 Sh (DS) & 0.1764 & 3.4589 & 516.55 \\
400-2 (DS) & 0.2582 & 3.7804 & 498.23 \\
401 Sh (DS) & 0.1707 & 5.0427 & 551.27 \\
401-2 (DS) & 0.2241 & 3.8891 & 470.27 \\
397 (DS) & 0.2354 & 3.6364 & 566.38 \\
397 (US) & 0.1933 & 4.1117 & 628.56 \\
\hline
\end{tabular}

Table 23: Fisheye Width and Length Versus Compression Strength from analySIS

\begin{tabular}{llll}
\hline & width, b [mm] & length, $\mathbf{l}$ [mm] & Fibre Image Analysis [MPa] \\
\hline K1-1 Sh (DS) & 0.2179 & 3.3294 & 442.34 \\
K1-2 (DS) & 0.2495 & 3.6269 & 432.35 \\
14-2 (DS) & 0.2074 & 4.9242 & 556.63 \\
14-2 (US) & 0.1319 & 5.1625 & 674.74 \\
11-1 Sh (DS) & 0.1675 & 5.6070 & $*$ \\
11-2 (DS) & 0.1701 & 5.7699 & 565.35 \\
11-2 (US) & 0.1721 & 6.8148 & 670.12 \\
400-1 Sh (DS) & 0.2556 & 4.5545 & 450.16 \\
400-2 (DS) & 0.2933 & 3.4372 & 440.75 \\
401 Sh (DS) & 0.1724 & 3.3464 & 471.63 \\
401-2 (DS) & 0.2960 & 3.5106 & 430.62 \\
397 (DS) & 0.2910 & 3.2446 & 434.53 \\
397 (US) & 0.2621 & 7.7038 & 658.14 \\
\hline
\end{tabular}

*censored data

Table 24: Fisheye Width and Length versus Compression Strength from Fibre Image Analysis 


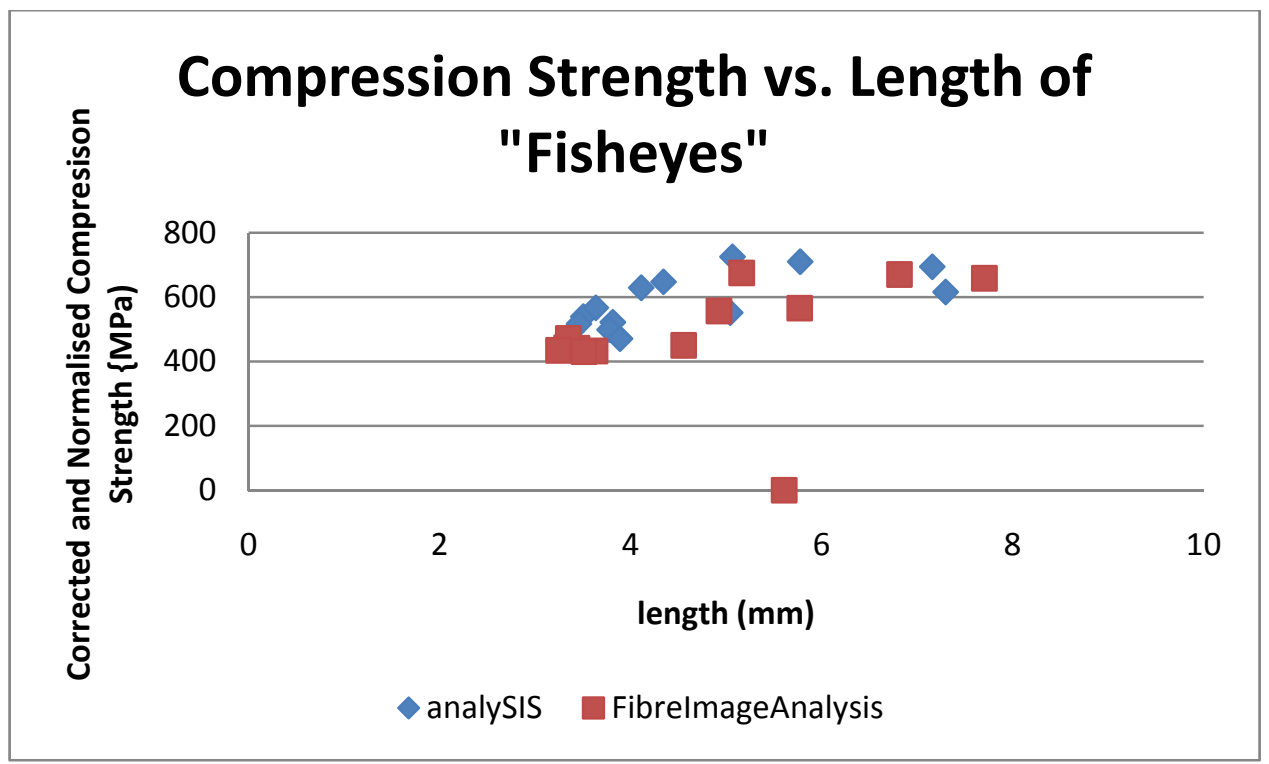

Figure 71: Scatter of Data from Table 23 and Table 24, Compression Strength versus Length of "Fisheyes"

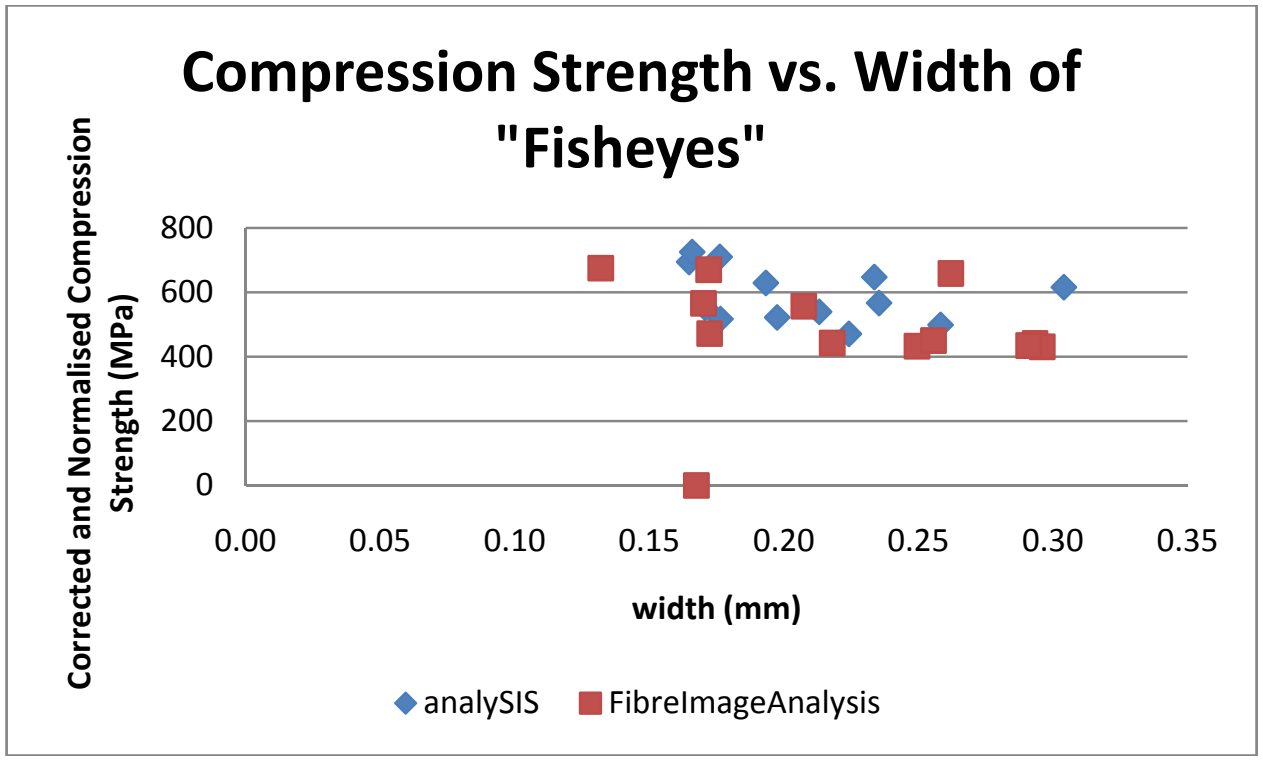

Figure 72: Scatter of Data from Table 23 and Table 24, Compression Strength versus Width of "Fisheyes" 


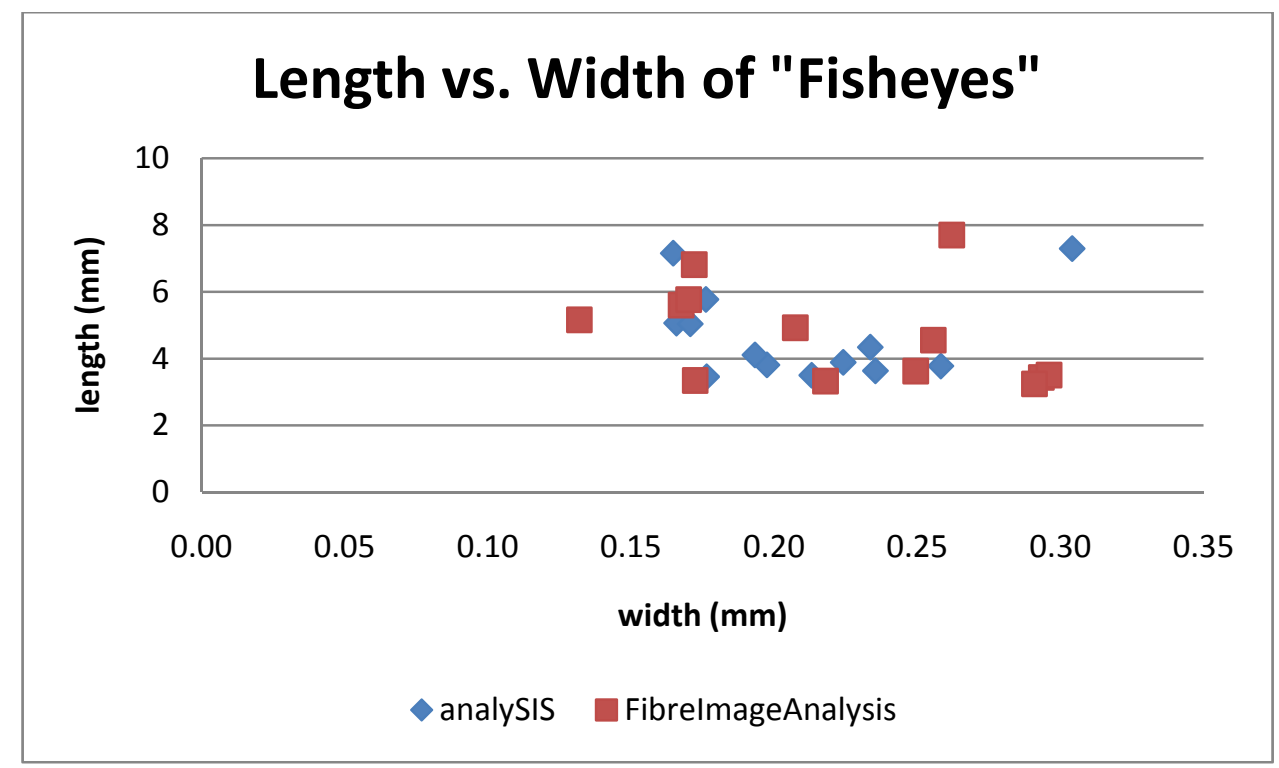

Figure 73: Scatter of Data from Table 23 and Table 24, Length versus Width of "Fisheyes"

\begin{tabular}{|c|c|c|c|c|c|c|c|}
\hline \multirow[t]{2}{*}{ CORRELATION: } & \multicolumn{3}{|c|}{ From analySIS } & \multicolumn{3}{|c|}{ From Fibre Image Analysis } & \multirow[b]{2}{*}{$\begin{array}{l}R \\
\text { value } \\
\text { (Ch. 5) }\end{array}$} \\
\hline & $\begin{array}{l}\text { width, } b \\
{[\mathrm{~mm}]}\end{array}$ & $\begin{array}{l}\text { length, } l \\
{[\mathrm{~mm}]}\end{array}$ & $\begin{array}{l}\text { analySIS } \\
\text { (Comp.) } \\
{[M P a]}\end{array}$ & $\begin{array}{l}\text { width, } b \\
\text { (FIA) } \\
{[\mathrm{mm}]}\end{array}$ & $\begin{array}{l}\text { length, } l \\
(F I A) \\
{[\mathrm{mm}]}\end{array}$ & $\begin{array}{l}\text { Fibre Image } \\
\text { Analysis } \\
\text { (Comp.) } \\
{[\mathrm{MPa}]}\end{array}$ & \\
\hline width, b [mm] & 1.00 & & & & & & \\
\hline length, 1 [mm] & 0.05 & 1.00 & & & & & \\
\hline $\begin{array}{l}\text { analySIS (Comp.) } \\
\text { [MPa] }\end{array}$ & -0.32 & 0.64 & 1.00 & & & & \\
\hline width, b (FIA) [mm] & 0.34 & -0.58 & -0.72 & 1.00 & & & \\
\hline length, I (FIA) [mm] & -0.32 & 0.38 & 0.78 & -0.38 & 1.00 & & \\
\hline $\begin{array}{l}\text { Fibre Image Analysis } \\
\text { (Comp.) [MPa] }\end{array}$ & 0.12 & 0.01 & 0.09 & -0.09 & 0.33 & 1.00 & \\
\hline$R$ value (Ch. 5) & -0.33 & 0.04 & 0.44 & -0.29 & 0.40 & -0.06 & 1.00 \\
\hline
\end{tabular}

Table 25: Correlation between R value, Compression Strength, Length, and Width of Fisheyes found with analySIS and Fibre Image Analysis, Data from Table 17, Table 23, and Table 24

From Table 25, it can be observed that there is a low positive correlation between the $R$ value (Ch. 5) and the length of "fisheyes" found with analySIS (0.04), a fairly high positive correlation found with the compression strength prediction from Fibre Image Analysis (0.44), and a fairly high positive correlation found with the length of "fisheyes" from Fibre Image Analysis 
(0.40). Also, like in Table 20, a fairly high positive correlation is observed between the $R$ value (Ch. 5) and the compression strength predictions with analySIS.

A fairly low negative correlation can be observed between the $R$ value (Ch. 5) and the width of "fisheyes" found with analySIS (-0.33) and with Fibre Image Analysis (-0.29).

\begin{tabular}{|c|c|c|c|}
\hline & \multicolumn{2}{|c|}{ Percent difference between Actual Results } & \\
\hline & analySIS & Fibre Image Analysis & $\begin{array}{l}\text { Std Deviation of Actual } \\
\text { Results [MPa] }\end{array}$ \\
\hline K1-1 Sh (DS) & 2.05 & 16.93 & 43.43 \\
\hline K1-2 (DS) & 1.77 & 18.38 & 59.83 \\
\hline 14-2 (DS) & 2.93 & 6.91 & 48.45 \\
\hline 14-2 (US) & 27.45 & 32.96 & 38.25 \\
\hline 11-1 Sh (DS) & 18.93 & $*$ & 8.13 \\
\hline 11-2 (DS) & 23.38 & 0.54 & 32.77 \\
\hline 11-2 (US) & 11.97 & 3.52 & 35.85 \\
\hline 400-1 Sh (DS) & 1.48 & 11.56 & 20.34 \\
\hline 400-2 (DS) & 6.55 & 17.33 & 21.85 \\
\hline $401 \mathrm{Sh}$ (DS) & 1.63 & 15.84 & 47.56 \\
\hline 401-2 (DS) & 35.69 & 41.11 & 19.37 \\
\hline 397 (DS) & 6.14 & 27.99 & 82.56 \\
\hline 397 (US) & 14.45 & 19.84 & 29.93 \\
\hline $\min$ & 1.48 & 0.54 & \\
\hline $\max$ & 35.69 & 41.11 & \\
\hline
\end{tabular}

*censored data

Table 26: Comparison between Percent Differene and Standard Deviation between Actual Compression Testing Results\

\begin{tabular}{|c|c|c|c|}
\hline & \multicolumn{2}{|c|}{$\begin{array}{l}\text { Percent difference between Actual } \\
\text { Results }\end{array}$} & \multirow[b]{2}{*}{$\begin{array}{l}\text { Std Deviation of Actual } \\
\text { Results }\end{array}$} \\
\hline & analySIS & $\begin{array}{l}\text { Fibre Image } \\
\text { Analysis }\end{array}$ & \\
\hline analySIS & 1.00 & & \\
\hline Fibre Image Analysis & 0.36 & 1.00 & \\
\hline $\begin{array}{l}\text { Std Deviation of Actual } \\
\text { Results [MPa] }\end{array}$ & -0.44 & 0.22 & 1.00 \\
\hline
\end{tabular}

Table 27: Correlation between Percentage Difference and Standard Deviation of Actual Results

A fairly high negative correlation between the standard deviation of the actual compression results and the percentage difference between analySIS and the actual compression results can be seen in Table $27(-0.44)$, whereas a fairly high correlation can be observed for Fibre Image Analysis (0.22). 


\subsection{DISCUSSION OF RESULTS}

Figure 68 shows the normalised and corrected compression strength predictions using SFI values from analySIS and SFI values from Fibre Image Analysis, compared to the laboratory results. Also, the percent difference can be seen on the same graph. A high percent difference can be observed with both analySIS and Fibre Image Analysis with the same textiles: 14-2 US and 401-2 DS. Also, a moderate percent difference can be observed for 397 (US) for both analySIS and Fibre Image Analysis. To some degree, these high percent differences can be explained. The names of these textiles are in "red" because they presented difficulties with Fibre Image Analysis and required "re-runs." These difficulties came in the form of really small and/or irregular "fisheyes," making the "fisheyes" hard to detect. There are other textiles that have moderate percent differences but they do not apply to both analySIS and Fibre Image Analysis at the same time.

Using two-factor ANOVA without replication, it can be seen from Table 19 that there is a difference between the textiles, with a $p$-value of 0.039949 , which is less than the significance level of 5\%. Also, the difference between the results analySIS and Fibre Image Analysis are moderate, given a significance level of 5\%. The $p$-value corresponding to the latter statement is larger than 0.05 . The $p$-value is 0.079521 , indicating a moderate level of truth in the statement.

The calculated $R$ value (Ch. 4) has a fairly strong positive correlation with the compression strength predictions with analySIS, as shown in Table 20. This indicates that the $R$ value (Ch. 4) is high when the prediction compression strength is high. Therefore, the R value (Ch. 4) is a good indicator of high compression strength predictions when using analySIS. However, a mild negative correlation can be seen between the $R$ value (Ch. 4) and the compression strength predictions using Fibre Image Analysis. Interestingly, a positive correlation can be seen between the $R$ value (Ch. 4) and the SFO. However, this cannot be seen when compared with the actual compression results from the laboratory. A fairly high negative correlation can be observed with respect with the $R$ value (Ch. 4) and the actual compression testing results. This means that a high $R$ value (Ch. 4) corresponds to low compression strength of the coupon. Also, a negative correlation can be seen between the $R$ value (Ch. 4) and the SFI values from both analySIS and Fibre Image Analysis. This makes sense because a high standard deviation of fibre misalignment angles corresponds to low reliability. 
From Figure 69, it can be seen that the SFI values have a large range of values, over a range of predicted compression strength values. It can be seen that Fibre Image Analysis tends to find higher SFI values, when compared to analySIS, as illustrated by the overwhelming number of red dots to the right of the graph.

In contrast with the SFI values, the SFO values and predicted compression strength, in Figure 70, show another pattern. Since the SFO values are the same for both analySIS and Fibre Image Analysis, the points representing Fibre Image Analysis are usually below those points representing analySIS. This means that Fibre Image Analysis usually has lower compression strength predictions. This conclusion can also be seen in Figure 68.

Figure 71 and Figure 73 show a scatter plot of the length and width of "fisheyes" compared to the predicted compression strength. It can be seen that the "fisheye" dimensions found using analySIS and Fibre Image Analysis are very dispersed amongst each other over the range of predicted compression strength and do not exhibit differences between analySIS and Fibre Image Analysis. In general, however, it can be seen that a very narrow width is often paired with a very long length, and a very wide width is paired with a very short length. This is noticeable in Figure 73 , where there are a large number of points only on the lower triangular end of the plot.

Table 25 shows that there is a fairly strong correlation between the lengths of "fisheyes" found using Fibre Image Analysis and the $R$ value (Ch. 4), and a mild positive correlation using analySIS. This means that a long "fisheye" length corresponds to a high $R$ value (Ch. 4). A fairly strong negative correlation between the widths of "fisheyes" and the $R$ value (Ch. 4 ) can be observed. This means that a wider "fisheye" corresponds to a low $R$ value (Ch. 4). Since the "fisheyes" are generally paired with a wide width and short length or a narrow width and a long length, as discussed in the previous paragraph, and that high $R$ values (Ch. 4) correspond with high compression strength predictions, it can be concluded that the "fisheyes" are long and narrow will have higher compression strength values, as can be seen in Figure 71.

The percentage difference between the predicted compression strength and the actual compression strength cannot be explained by corresponding error in the laboratory, as can be determined by the standard deviation of the compression strength of the coupons. This is shown in Table 26 and Table 27. A fairly high negative correlation can be observed between the 
standard deviation of the actual compression results and the percentage difference for analySIS and a fairly positive correlation can be observed for Fibre Image Analysis.

\subsection{SUMMARY OF CONTRIBUTIONS}

- Studied MWK CFRP textiles and composites made from these textiles. There were eight carbon fibre textiles: six 0/90 (four of six were sheared), one 135/0/45/90, and one 45/0/135.

- SFI, SFO, and width of length of "fisheyes" for each textile were found.

- SFI and "fisheye" values came from two sources: analySIS software or Fibre Image Analysis software.

- SFI with analySIS: increased sample size.

- "Fisheyes" with analySIS: modified VBA macros to deal with curved carbon fibre particles.

- Fibre Image Analysis software was created by the Faserinstitut Bremen (FIBRE) e.V. (Bremen Fibre Institute) at the University of Bremen in Germany and was never used before for compression strength predictions.

- The compression strength was found using a finite element model created in MSC Patran (modified FMB-PMB model).

- A reliability model was created to find the reliability, $R$, of each textile, based on the angle matrix used to find the SFI with Fibre Image Analysis and on the distribution of actual compression results.

\subsection{FUTURE WORK}

Future work can be done to better improve how the SFI value used in the compression strength prediction. As indicated in Section 5.1 in Chapter 5, a SFI value that is approximately over three would yield the same result in the FMB model. Despite that a high level of resolution of the possible SFI values can be obtained through Fibre Image Analysis using 2400 dpi images, where the resolution of SFI values describes the quality of the textile, this higher resolution often means a higher SFI value, a value over three that cannot be analysed using the FMB model.

Also, it can also be observed that depending on the size of the domain chosen in Fibre Image Analysis and the sharpness of the scanned image, the SFI value could have a range of values. For this study, the domain size was fixed for all analysed images, fixed at $50 \times 50$ pixels 
with Fibre Image Analysis and fixed at 80 x 80 pixels for analySIS for consistency in the results. However, a method or a rule that can be used to determine the optimal domain size for microscope images and scanned images would greatly assist further study.

In order for the SFI and "fisheye" dimensions to be used as part of an on-line quality control process, a way of reducing processing requirements or increasing computer processing speed would be main determining factor of success. Some rudimentary work has been done in this study on processing speed, but a way to properly understand what affects these processing requirements would need to be further researched. Also, the three-dimensional waviness factor, SFO, has not been studied with respect to on-line quality control. Therefore, a way to include it would also be beneficial.

For "fisheyes," the interpolation model in "Parametric Study with Unit Cell Model" can also be improved if it accepted a larger range of values.

For the reliability model, the $R$ value (Ch. 4) currently only uses the angle matrix, used to find the SFI, from Fibre Image Analysis and the actual compression strength found from the coupons. A way to expand this model to include the three-dimensional waviness factor, SFO, may make the $R$ value (Ch. 4) calculation more accurate. A GUI that could tie all of the calculations in MATLAB would also speed up calculations. Since the $R$ value (Ch. 4) is currently being calculated using actual compression strength results, thus destructive testing would be required; the current model cannot be used as an on-line quality assessment tool. A way to bypass this problem would be to determine a probability density function (PDF) that simulates the actual compression results given the combination of fibre and resin. Therefore, the only "input" data would be the scanned area of the textile. Further study would be required to determine this PDF for a particular combination of fibre and resin.

As described in Chapter 5, the predicted compression strength predictions from the FMBPMB Unit Cell Model was "normalised" to the thickness of the laminate, according to Classical Laminate Theory (CLT). It must be noted here that formula for the effective transverse modulus, $E_{2}^{*}$, would result in an under estimation of the compression strength. This is a limitation of this study and future improvements would involve using the correct transverse modulus value. 


\subsection{CONCLUSION}

The historical and theoretical background behind MWK CFRP textiles and composites has been thoroughly described with respect to defect and the calculation of the compression strength. For this study, a select number of textiles have been studied, finding the SFI, SFO, and width of length of "fisheyes" for each textile. The SFI and "fisheye" values came from two sources: analySIS software or Fibre Image Analysis. This data was then used to find the compression strength using a finite element model created in MSC Patran and a Unit Cell Model. A reliability model was created to find the reliability, $R$ (Ch. 4), of each textile, based on the angle matrix used to find the SFI with Fibre Image Analysis and based on the distribution of actual compression results.

It has been found that the predicted compression strength from analySIS and Fibre Image Analysis yielded very similar results and that the $R$ value (Ch. 4) was a good predictor of high predicted compression strength with analySIS. However, there is a large percentage of difference between the predicted and the actual compression strength for some textiles. This can be attributed to the inherent lack of regularity for some of the examined textiles, variability in determining the SFI and "fisheye" parameters, and limitations with the FMB-PMB and Unit Cell Models

Future improvements would involve devising rules and methods to determine the SFI and "fisheye" parameters, modifying the FMB-PMB and Unit Cell Models, and making the analysis process faster and more applicable for on-line quality process control. 


\section{REFERENCES}

Argon, A. S. (1972). Fracture of composites. In H. Herman, Treatise on materials science and technology (Vol. 1, pp. 79-114). New York: Academic Press.

Armstrong, K. B., \& Barrett, R. T. (1998). Care and repair of advanced composites. Warendale, PA, U.S.A.: Society of Automotive Engineers, Inc.

Banks, J., Carson II, J. S., Nelson, B. L., \& Nicol, D. M. (2005). Discrete-event system simulation (4 ed.). Upper Saddle River, New Jersey: Pearson Prentice Hall.

Barbero, E. J. (2008). Finite element analysis of composite materials. Boca Raton, FL, U.S.A.: Taylor \& Francis Group.

Barbero, E. J. (1998). Prediction of compression strength of unidirectional polymer matrix composites. Journal of Composite Materials , 32 (5), 483-502.

Berg, C. A., \& Salama, M. (1973). Fatigue of graphite fiber-reinforced epoxy in compression. Fiber Sci. Tech. , 6 (2), 79-113.

Bibo, G. A., Hogg, P. J., \& Kemp, M. (1997). Mechanical characterisation of glass- and carbonfibre-reinforced composites made with non-crimp fabrics. Composites Science and Technology, $57(9-10), 1221-1241$.

Brönnimann, R., Nellen, P. M., \& Sennhauser, U. (2000). Reliability monitoring of cfrp structural elements in bridges with fiber optic bragg grating sensors. Journal of Intelligent Material Systems and Structures , 10 (4), 322-329.

Budiansky, B., \& Fleck, N. A. (1993). Compressive failure of fibre composites. Journal of the Mechanics and Physics of Solids , 41 (1), 183-211.

Chaplin, C. R. (1977). Compressive fracture in unidirectional glass reinforced plastics. J. Mat. Sci. , 12 (2), 347-352.

Creighton, C. J., Sutcliffe, M. P., \& Clyne, T. W. (2001). A multiple field image analysis procedure for characterisation of fibre alignment in composites. Composites Part A: Applied Science and Manufacturing , 32 (2), 221-229.

Daniel, I. M., \& Ishai, O. (2006). Engineering mechanics of composite materials (2 ed.). New York: Oxford University Press.

Evans, A. G., \& Adler, W. F. (1978). Kinking as a mode of structural degradation in carbon fiber composites. Acta Metallurgica , 26 (5), 725-738.

Herakovich, C. T. (1998). Mechanics of fibrous composites. John Wiley \& Sons, Inc. 
Heß, H., Roth, Y. C., \& Himmel, N. (2007). Elastic constants estimation of stitched NCF CFRP laminates based on a finite element unit-cell model. Composites Science and Technology, 67 (6), 1081-1095.

Hörsting, K., Wulhorst, B., Franzke, G., \& Offermann, P. (1993). New types of textile fabrics for fiber composites. SAMPE 1. 29, pp. 7- 12. SAMPE.

Hu, J., \& Xin, B. (2004). Image based modeling and analysis of textile materials. In D. Zhang, M. Kamel, \& G. Baciu, Integrated image and graphics technologies (pp. 283-307). Kowloon, Hong Kong: Springer Netherlands.

Kuntz, J. (2010, September 20). Waviness (SFO). Applied methods for textile characterization . Bremen, Germany.

Lam, P. N. (2008). Entwicklung eines rechentools zur abschätzung der mechanischen eigenschaften von laminaten aus multiaxialgelegen unter einbeziehung verschiedener abschätzungsmethoden zum einfluss von inhomogenitäten im textilen halbzeug. Diploma thesis, Institut für Verbundwerkstoffe, Technische Universität Kaiserslautern.

Leaver, H. R., \& Thomas, T. R. (1974). Analysis and presentation of experimental results. New York: The Macmillian Press Ltd.

Lehners, F. (2008). Zusammenhang zwischen inhomogenitäten in trockenen textilen halbzeugen und welligkeiten im faserverbundlaminat. Diploma thesis, Institut für Flugzeugbau und Leichtbau Technische Universität Braunschweig, Bremen, Germany.

Madhukar, M. S., \& Drzal, L. T. (1992). Fiber-matrix adhesion and its effect on composite mechanical properties. III. Longitudinal (0 degree) compressive properties of graphite/epoxy composites. J. Comp. Mat. , 26 (3), 310-333.

Mallick, P. K. (1997). Composites engineering handbook. New York: Marcel Dekker, Inc.

MathWorks help. (1984-2011). (MathWorks) Retrieved January 9, 2011, from MathWorks web site: http://www.mathworks.com/help/techdoc/ref/f16-6011.html

Meeker, W. Q., \& Escobar, L. A. (1998). Statistical methods for reliability data. New York: Wiley.

Miene, A., Herrmann, A. S., \& Göttinger, M. (2008). Quality assurance by digital image analysis for the preforming and draping process of dry carbon fiber material. SAMPE Europe 29th SEICO Conference. Paris, France: SAMPE.

Miller, A. J. (1996). The effect of microstructural parameters on the mechanical properties of non-crimp fabric composites. M.Phil thesis, Cranfield University. 
Montgomery, D. C. (2005). Design and analysis of experiments (6 ed.). United States of America: John Wiley \& Sons, Inc.

Naik, N. K., \& Kumar, R. S. (1999). Compressive strength of unidirectional composites: evaluation and comparison of prediction models. Composite Structures , 46 (3), 299-308.

Pansart, S. (2008). A comprehensive explanation of compression strength differences between various CFRP materials through a novel micro-meso model. Otto-von-Guericke-Universität Magdeburg. Köln: DLR, Dt. Zentrum für Luft- und Raumfahrt.

Pourdeyhimi, B., \& Kim, H. S. (2002). Measuring fiber orientation in nonwovens: the hough transform. Textile Research Journal , 72 (9), 803-809.

Pourdeyhimi, B., Dent, R., Jerbi, A., Tanaka, S., \& Deshpande, A. (1999). Measuring fiber orientations in nonwovens: part v: real webs. Textile Research Journal , 69 (3), 185-192.

Pourdeyhimi, B., Ramanathan, R., \& Dent, R. (1996a). Measuring fiber orientation in nonwovens: part I: simulation. Textile Research Journal , 66 (11), 713-722.

Pourdeyhimi, B., Ramanathan, R., \& Dent, R. (1996b). Measuring fibre orientation in nonwovens: part II: direct tracking. Textile Research Journal , 66 (12), 747-753.

Rabotnov, Y. N., Tupolev, A. A., Kut'inov, V. F., Kogaev, V. P., Berezin, A. V., \& Sul, V. V. (1982). Use of carbon fiber-reinforced plastics in aircraft construction. Plenum Publishing Corporation, 17 (4), 461-462.

Rantala, J., Wu, D., \& Busse, G. (1995). Amplitude-modulated lock-in vibrothermography for NDE of polymers and composites. Research in Nondestructive Evaluation , 7 (4), 215-228.

Rao, S. S. (1992). Reliability-based Design. USA: McGraw-Hill, Inc.

Rehbein, J. (2009). Charakteristika trockener multiaxialgelege in korrelation mit den mechanischen eigenschaften der daraus hergestellten faserverbundwerkstoffe für die luftfahrtindustrie. Diploma thesis, RWTH Aachen University, Bremen, Germany.

Rosen, B. W. (1965). Mechanics of composite strengthening. In Fiber composite materials. Metals Park, Ohio: ASM.

Schapery, R. A. (1991). Analysis of local buckling in viscoelastic composites. IUTAM Symposium on Local Mechanics Concepts for Composite Material Systems, (pp. 229-250). Blacksburg, Virginia, USA.

Schneider, M., Edelmann, K., \& Tiltmann, U. (2004). Quality analysis of reinforcement structures for composites by digital image processing. 25th International SAMPE Europe Conference. Paris, France: SAMPE. 
Schultheisz, C. R., \& Waas, A. M. (1996). Compressive failure of composites, part i testing and micromechanical theories. Progress in Aerospace Sciences , 32 (1), 1-42.

Spragg, C. J., \& Drzal, L. T. (1996). Fiber, matrix, and interface properties. West Conshocken, PA, U.S.A.: Americal Society for Testing and Materials.

Steif, P. S. (1990a). A model for kinking in fiber composites: Part I-Fiber breakage via microbuckling. Int. J. Solids Struct. , 26 (5-6), 549-561.

Steif, P. S. (1990b). A model for kinking in fiber composites: Part II-Kink band formation. Int. J. Solids Struct. , 26 (5-6), 563-569.

Tamura, H., Mori, S., \& Yamawaki, T. (1978). Textural features corresponding to visual perception. IEEE Transactions on Systems, Man, and Cybernetics , 8 (6), 460-473.

Thurstone, L. L. (1927). A law of comparative judgement. Psychol. Rev. , 34 (4), 273-286.

Todoroki, A. (2008). Delamination monitoring analysis of CFRP structures using multi-probe electrical method. Journal of Intelligent Material Systems and Structures , 19 (3), 291-298.

Todoroki, A., Hirai, N., \& Matsuzaki, R. (2008). Electrical resistance change method for delamination of CFRP plates: effect of plate scale. 6934. SPIE.

Wasserman, G. S. (2003). Reliability verification, testing, and analysis in engineering design. New York, NY: Marcel Dekker, Inc.

Weaver, C. W., \& Williams, J. G. (1975). Deformation of a carbon-epoxy composite under hydrostatic pressure. J. Mat. Sci. , 10 (8), 1323-1333.

Whitney, J. M., Daniel, I. M., \& Pipes, R. B. (1984). Experimental mechanics of fiber reinforced composite materials, revised edition. Society for Experimental Mechanics.

Wietgrefe, M. (2007). Finite-elemente-modellierung des druckversagens in kohlefaserverbundwerkstoffen. Diploma thesis, Technical University of Braunschweig, Bremen, Germany.

Wronski, A. S., \& Parry, T. V. (1982). Compressive failure and kinking in uniaxially aligned glass-resin composite under superposed hydrostatic pressure. J. Mat. Sci. , 17 (12), 3656-3662.

Yurgartis, S. W. (1987). Measurement of small angle fiber misalignments in continuous fiber composites. Composites Science and Technology, 30 (4), 279-293. 


\section{APPENDICES}

\section{A. FORMULA FOR THE SAMPLE STANDARD DEVIATION OF TWO INDEPENDENT SAMPLES}

The derivations were performed by the author of this thesis.

Independent sampling means that the samples which are selected have no effect on one another.

\section{Given:}

$N_{a} \quad$ Number of items in sample $A$

$N_{b} \quad$ Number of items in sample $B$

$N_{t} \quad=N_{a}+N_{b}$

$\Sigma a \quad$ Sum of items in sample $A$

$\Sigma b \quad$ Sum of items in sample $B$

$\Sigma a^{2} \quad$ Sum of squares of items in sample $A$

$\Sigma b^{2} \quad$ Sum of squares of items in sample $B$

$\mu_{a} \quad$ Sample mean of sample $A$

$\mu_{b} \quad$ Sample mean of sample $B$

$\mu_{t} \quad$ Sample mean of combined sample

$\sigma_{a} \quad$ Sample standard deviation of sample $A$

$\sigma_{b} \quad$ Sample standard deviation of sample $B$

$\sigma_{t} \quad$ Sample standard deviation of combined sample

$$
\begin{aligned}
& \Sigma t=\Sigma a+\Sigma b \\
& \Sigma \mathrm{t}^{2}=\Sigma \mathrm{a}^{2}+\Sigma \mathrm{b}^{2} \\
& \mu_{a}=\frac{\sum a}{N_{a}} \\
& \mu_{b}=\frac{\sum b}{N_{b}}
\end{aligned}
$$




$$
\mu_{t}=\frac{\sum t}{N_{t}}
$$

A.v

Derived from A.i,

$$
\mu_{t}=\frac{\left(N_{a} \bullet \mu_{a}+N_{b} \bullet \mu_{b}\right)}{N_{t}}
$$

Sample standard deviation, with a correction of $(N-1)$ for an unbiased estimator of variance is defined as (See note at the end of this document), where $B$ is the correction:

$$
\sigma_{a}{ }^{2}=B\left(\sum_{1}^{N} \frac{a^{2}}{N_{a}}-\mu_{a}^{2}\right) \quad \text { OR } \sigma_{b}^{2}=B\left(\sum_{1}^{N} \frac{b^{2}}{N_{b}}-\mu_{b}^{2}\right)
$$

Finding $\Sigma a^{2}$

$$
\begin{aligned}
& \frac{\sigma_{a}^{2}}{B}=\left(\sum_{1}^{N} \frac{a^{2}}{N_{a}}-\mu_{a}^{2}\right) \\
& \frac{\sigma_{a}^{2}}{B} N_{a}=\sum_{1}^{N} a^{2}-N_{a} \mu_{a}^{2} \\
& \sum_{1}^{N} a^{2}=\frac{\sigma_{a}^{2}}{B} N_{a}+N_{a} \mu_{a}^{2} \\
& \sum_{1}^{N} a^{2}=\sigma_{a}{ }^{2}\left(N_{a}-1\right)+N_{a} \mu_{a}^{2}
\end{aligned}
$$

This process can be repeated for $b$. Therefore,

$$
\sum_{1}^{N} b^{2}=\sigma_{b}^{2}\left(N_{b}-1\right)+N_{b} \mu_{b}^{2}
$$

From A.i, A.ii, A.viii, and A.ix:

$$
\begin{aligned}
\Sigma t^{2} & =\sigma_{a}^{2}\left(N_{a}-1\right)+\sigma_{b}^{2}\left(N_{b}-1\right)+N_{a} \mu_{a}^{2}+N_{b} \mu_{b}^{2} \\
\sigma_{t}^{2} & =B\left(\sum_{1}^{N} \frac{t^{2}}{N_{t}}-\mu_{t}^{2}\right) \\
& =\frac{B}{N_{t}}\left(\sigma_{a}{ }^{2}\left(N_{a}-1\right)+\sigma_{b}^{2}\left(N_{b}-1\right)+N_{a} \mu_{a}^{2}+N_{b} \mu_{b}^{2}\right)-B \mu_{t}^{2} \\
& =\frac{1}{N_{t}-1}\left(\sigma_{a}{ }^{2}\left(N_{a}-1\right)+\sigma_{b}{ }^{2}\left(N_{b}-1\right)+N_{a} \mu_{a}^{2}+N_{b} \mu_{b}^{2}\right)-N_{t} \frac{\mu_{t}^{2}}{N_{t}-1}
\end{aligned}
$$




$$
\sigma_{t}^{2}=\frac{\sigma_{a}^{2}\left(N_{a}-1\right)+\sigma_{b}^{2}\left(N_{b}-1\right)+N_{a} \mu_{a}^{2}+N_{b} \mu_{b}^{2}-N_{t} \mu_{t}^{2}}{N_{t}-1}
$$

A.x

NOTE (Leaver \& Thomas, 1974):

Equation A.vii is from

$$
\sigma^{2}=\frac{(x-\bar{x})^{2}}{N}
$$

This is multiplied by $N /(N-1)$ because the degree of freedom is $N-1$ in a sample size of $N$.

$$
\sigma^{2}=\frac{\sum(x-\bar{x})^{2}}{N-1}
$$

Where,

$$
\begin{aligned}
& \sum_{1}^{n}(x-\bar{x})^{2} \\
& =\sum_{1}^{N}\left(x^{2}-2 x \bar{x}+\bar{x}^{2}\right) \\
& \quad=\sum_{1}^{N} x^{2}-\sum_{1}^{N} 2 x \bar{x}+\sum_{1}^{N} \bar{x}^{2} \\
& \quad=\sum_{1}^{N} x^{2}-2 \bar{x} \sum_{1}^{N} x+\sum_{1}^{N} \bar{x}^{2} \\
& \frac{\sum_{1}^{N} x}{N}, \\
& \quad=\sum_{1}^{N} x^{2}-2 \bar{x}(\bar{x} N)+N \bar{x}^{2} \\
& \quad=\sum_{1}^{N} x^{2}-2 N \bar{x}^{2}+N \bar{x}^{2} \\
& \quad=\sum_{1}^{n} x^{2}-N \bar{x}^{2}
\end{aligned}
$$

From $\bar{x}=\frac{\sum_{1}^{N} x}{N}$,

Therefore,

$$
\begin{aligned}
\sigma^{2} & =\frac{\sum(x-\bar{x})^{2}}{N}\left(\frac{N}{N-1}\right) \\
& =\frac{\sum_{1}^{n} x^{2}-N \bar{x}^{2}}{N}\left(\frac{N}{N-1}\right)
\end{aligned}
$$




$$
=\left(\sum_{1}^{N} \frac{x^{2}}{N}-\bar{x}^{2}\right)\left(\frac{N}{N-1}\right)
$$

Let $\mathrm{B}=\mathrm{N} /(\mathrm{N}-1)$,

$$
=\left(\sum_{1}^{N} \frac{x^{2}}{N}-\bar{x}^{2}\right) B
$$




\section{B. MODIFIED FORMULAS FOR THE SAMPLE STANDARD DEVIATION OF TWO INDEPENDENT SAMPLES}

\section{MICROSCOPE IMAGES}

The derivations were performed by the author of this thesis. The following is an example.

\begin{tabular}{|c|c|c|c|c|}
\hline & $\begin{array}{l}\text { Angle Matrix } \\
\text { Size }\end{array}$ & $\begin{array}{l}\text { Count of Actual Values, } \\
N\end{array}$ & $\begin{array}{l}\text { Average Angle, } \\
\mu\end{array}$ & $\begin{array}{l}\text { Standard Deviation, } \\
\sigma\end{array}$ \\
\hline 11-2_1_DS & $28 \times 21$ & 0 & 0 & 0 \\
\hline 11-2_1_DS_trial2 & $28 \times 21$ & 63 & 47.178 & 1.652 \\
\hline $11-2 \_2 \_D S$ & $28 \times 21$ & 14 & 43.675 & 2.96 \\
\hline 11-2_2_DS_trial2 & $28 \times 21$ & 1 & 46.331 & 0 \\
\hline 11-2_3_DS & $28 \times 21$ & 220 & 43.518 & 2.842 \\
\hline 11-2_3_DS_trial2 & $28 \times 21$ & 2 & 46.349 & 1.613 \\
\hline 11-2_4_DS & $28 \times 21$ & 581 & 47.21 & 1.591 \\
\hline 11-2_4_DS_trial2 & $28 \times 21$ & 38 & 48.015 & 1.774 \\
\hline 11-2_5_DS & $28 \times 21$ & 19 & 45.392 & 1.493 \\
\hline 11-2_5_DS_trial2 & $28 \times 21$ & 8 & 45.607 & 1.898 \\
\hline 11-2_6_DS & $28 \times 21$ & 9 & 46.316 & 1.759 \\
\hline 11-2_6_DS_trial2 & $28 \times 21$ & 34 & 45.281 & 1.908 \\
\hline 11-2_7_DS & $28 \times 21$ & 21 & 48.706 & 2.654 \\
\hline 11-2_7_DS_trial2 & $28 \times 21$ & 108 & 44.95 & 1.899 \\
\hline
\end{tabular}

*Bold numbers represent the microscope image number. Seven microscope images were taken for each trial and there were two trials.

Domain size is 50x50 pixels for all

\section{$\underline{\text { Only Trial } 1}$}

$$
\begin{aligned}
& \mu \mathrm{t}: \quad 46.1997 \\
& \mu_{t}=\frac{\left(N_{a} \bullet \mu_{a}+\ldots+N_{g} \bullet \mu_{g}\right)}{N_{t}} \\
& \text { SFI, } \\
& \sigma_{\mathrm{t}}^{2}: \quad 6.279452 \\
& \sigma_{t}^{2}=\frac{\sigma_{a}^{2}\left(N_{a}-1\right)+\ldots+\sigma_{g}{ }^{2}\left(N_{g}-1\right)+N_{a} \mu_{a}^{2}+\ldots+N_{g} \mu_{g}{ }^{2}-N_{t} \mu_{t}^{2}}{N_{t}-1}
\end{aligned}
$$

SFI,

$\sigma_{\mathrm{t}}: \quad 2.505883$

\section{Only Trial 2}

$\mu \mathrm{t}: \quad 46.04261$

$$
\mu_{t}=\frac{\left(N_{a} \bullet \mu_{a}+\ldots+N_{g} \bullet \mu_{g}\right)}{N_{t}}
$$


$\begin{array}{ll}\mathrm{SFI}, & \sigma_{t}{ }^{2}=\frac{\sigma_{a}{ }^{2}\left(N_{a}-1\right)+\ldots+\sigma_{g}{ }^{2}\left(N_{g}-1\right)+N_{a} \mu_{a}{ }^{2}+\ldots+N_{g} \mu_{g}{ }^{2}-N_{t} \mu_{t}{ }^{2}}{\sigma_{t}:}\end{array}$

SFI,

$\sigma_{\mathrm{t}}: \quad 2.17691$

All

$\mu \mathrm{t}: \quad 46.16401$

$\mu_{t}=\frac{\left(N_{a} \bullet \mu_{a}+\ldots+N_{n} \bullet \mu_{n}\right)}{N_{t}}$

SFI,

$\sigma_{\mathrm{t}}^{2}: \quad 6.418329$

$$
\sigma_{t}^{2}=\frac{\sigma_{a}^{2}\left(N_{a}-1\right)+\ldots+\sigma_{n}^{2}\left(N_{n}-1\right)+N_{a} \mu_{a}^{2}+\ldots+N_{n} \mu_{n}^{2}-N_{t} \mu_{t}^{2}}{N_{t}-1}
$$

SFI,

$\sigma_{t}: \quad \mathbf{2 . 5 3 3 4 4 2}$

\section{2400 DPI IMAGES}

For textile 11-2 DS:

A

First Sample (Top Left)

Number of Samples, Na:

Standard Deviation, бa:

Average, $\mu$ a:

\begin{tabular}{|l|}
\hline 5876 \\
\hline 4.32 \\
\hline 0.277 \\
\hline
\end{tabular}

B

Second Sample (Top Right)

Number of Samples, $\mathrm{Nb}$ :

Standard Deviation, $\sigma \mathrm{b}$ :

Average, $\mu \mathrm{b}$ :

\begin{tabular}{|l|}
\hline 5721 \\
\hline 4.492 \\
\hline 0.217 \\
\hline
\end{tabular}

C

Third Sample (Bottom Right)

Number of Samples, $\mathrm{N}_{\mathrm{c}}$ :

Standard Deviation, $\sigma c$ :

Average, $\mu_{\mathrm{c}}$ :

\begin{tabular}{|l|}
\hline 5847 \\
\hline 4.267 \\
\hline-0.459 \\
\hline
\end{tabular}

$179.541\left\{\begin{array}{l}\text { Original angle value is subtracted } \\ \text { from } 180 \text { to normalise angle values } \\ \text { to } 0^{\circ} .\end{array}\right.$

D

Fourth Sample (Bottom Left)

Number of Samples, $\mathrm{N}_{\mathrm{d}}$ :

Standard Deviation, od:

\begin{tabular}{|l|}
\hline 5668 \\
\hline 4.235 \\
\hline
\end{tabular}


Average, $\mu \mathrm{d}$ :

$\mu \mathrm{t}:$

\subsection{5}

$0.036222 \quad \mu_{t}=\frac{\left(N_{a} \bullet \mu_{a}+N_{b} \bullet \mu_{b}+N_{c} \bullet \mu_{c}+N_{d} \bullet \mu_{d}\right)}{N_{t}}$

SFI, $\sigma_{\mathrm{t}}^{2}$ :

18.82807

$\sigma_{t}^{2}=\frac{\sigma_{a}{ }^{2}\left(N_{a}-1\right)+\sigma_{b}{ }^{2}\left(N_{b}-1\right)+\sigma_{c}{ }^{2}\left(N_{c}-1\right)+\sigma_{d}{ }^{2}\left(N_{d}-1\right)+N_{a} \mu_{a}{ }^{2}+N_{b} \mu_{b}{ }^{2}+N_{c} \mu_{c}{ }^{2}+N_{d} \mu_{d}{ }^{2}-N_{t} \mu_{t}{ }^{2}}{N_{t}-1}$

SFI, $\sigma_{t}$ :

4.339133 


\section{NORMALISATION USING CLASSICAL LAMINATION THEORY}

The formulas below are from the book by Herakovich (1998). The numbers in brackets refer to the corresponding equation number in the book.

\section{Notation:}

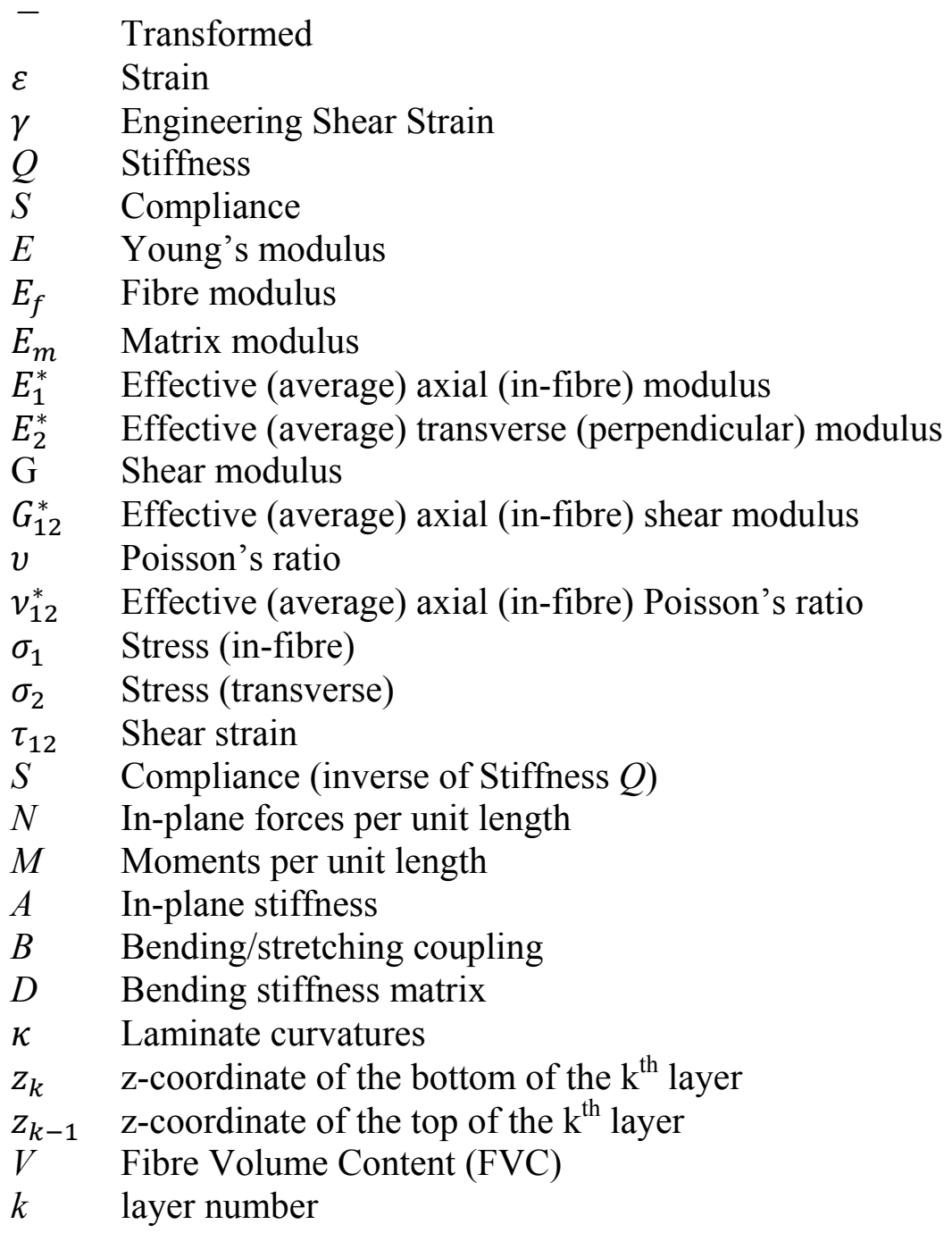

\section{Step 1}

$$
\left\{\begin{array}{c}
\varepsilon_{1} \\
\varepsilon_{2} \\
\gamma_{12}
\end{array}\right\}=\left[\begin{array}{ccc}
S_{11} & S_{12} & 0 \\
S_{12} & S_{22} & 0 \\
0 & 0 & S_{66}
\end{array}\right]\left\{\begin{array}{c}
\sigma_{1} \\
\sigma_{2} \\
\tau_{12}
\end{array}\right\}
$$

Let

$\sigma_{1}$ be the estimated compression value of an UD ply in fibre direction from the FMB-PMB Unit Cell model.

$\sigma_{2}=0$ because there is no transverse stress 
$\tau_{12}=0$ because there is no shear stress

Then let

$\left\{\begin{array}{c}\varepsilon_{x}^{0} \\ \varepsilon_{y}^{0} \\ \gamma_{x y}^{0}\end{array}\right\}=\left\{\begin{array}{c}\varepsilon_{1} \\ \varepsilon_{2} \\ \gamma_{12}\end{array}\right\}$

\section{Step 2}

$\left\{\begin{array}{c}N_{x} \\ N_{y} \\ N_{x y}\end{array}\right\}=\left[\begin{array}{lll}A_{11} & A_{12} & A_{16} \\ A_{12} & A_{22} & A_{26} \\ A_{16} & A_{26} & A_{66}\end{array}\right]\left\{\begin{array}{c}\varepsilon_{x}^{0} \\ \varepsilon_{y}^{0} \\ \gamma_{x y}^{0}\end{array}\right\}$

Here, the $A$ matrix has already accounted for rotation, $\Theta$, according to Figure 65 , Figure 66 , and Figure 67. How $A$ is calculated will be described later. Only $N_{x}$ is needed. It has units of force per unit length and corresponds to when fibres in the test direction fail and when the UD ply fails in the model.

\section{Step 3}

Find $X$

$X=\frac{N_{x}}{\text { nominal laminate thickness at } 60 \% \text { FVC (calculated thickness) }}$

The calculated thickness is according to equation 3.2 in Section 3.3 in Chapter 3, with $m_{a}=0$, $n=1$, and not multiplying by $100 \%$.

In Step 1,

$\mathrm{S}$ is found by:

$$
\left\{\begin{array}{c}
\varepsilon_{1} \\
\varepsilon_{2} \\
\gamma_{12}
\end{array}\right\}=\left[\begin{array}{ccc}
S_{11} & S_{12} & 0 \\
S_{12} & S_{22} & 0 \\
0 & 0 & S_{66}
\end{array}\right]\left\{\begin{array}{c}
\sigma_{1} \\
\sigma_{2} \\
\tau_{12}
\end{array}\right\}
$$

Where

$S_{11}=\frac{1}{E_{1}}$

$S_{12}=\frac{-v_{21}}{E_{2}}$

$S_{21}=\frac{-v_{12}}{E_{1}}$

$S_{22}=\frac{1}{E_{2}}$

$S_{66}=\frac{1}{G_{12}}$ 


\section{In Step 2,}

Matrix $A$ is found by:

$$
\left\{\begin{array}{c}
N_{x} \\
N_{y} \\
N_{x y} \\
M_{x} \\
M_{y} \\
M_{x y}
\end{array}\right\}=\left[\begin{array}{cccccc}
A_{11} & A_{12} & A_{16} & B_{11} & B_{12} & B_{16} \\
A_{12} & A_{22} & A_{26} & B_{12} & B_{22} & B_{26} \\
A_{16} & A_{26} & A_{66} & B_{16} & B_{26} & B_{66} \\
B_{11} & B_{12} & B_{16} & D_{11} & D_{12} & D_{16} \\
B_{12} & B_{22} & B_{26} & D_{12} & D_{22} & D_{26} \\
B_{16} & B_{26} & B_{66} & D_{16} & D_{26} & D_{66}
\end{array}\right]\left\{\begin{array}{c}
\varepsilon_{x}^{0} \\
\varepsilon_{y}^{0} \\
\gamma_{x y}^{0} \\
\kappa_{x} \\
\kappa_{y} \\
\kappa_{x y}
\end{array}\right\}
$$

However, since there is no bending and the laminate is symmetric, 5.23 reduces to:

$$
\left\{\begin{array}{c}
N_{x} \\
N_{y} \\
N_{x y}
\end{array}\right\}=\left[\begin{array}{lll}
A_{11} & A_{12} & A_{16} \\
A_{12} & A_{22} & A_{26} \\
A_{16} & A_{26} & A_{66}
\end{array}\right]\left\{\begin{array}{c}
\varepsilon_{x}^{0} \\
\varepsilon_{y}^{0} \\
\gamma_{x y}^{0}
\end{array}\right\}
$$

Where,

$$
[A]=\sum_{k=1}^{N}[\bar{Q}]^{k}\left(z_{k}-z_{k-1}\right)
$$

Where $\bar{Q}$ is the plane stress transformed reduced stiffness matrix from Section 4.3: 2D

Transformations about an Axis in Herakovich (1998). Here, $z_{k}-z_{k-1}$ is the thickness of the layer calculated from equation 3.2 in Section 3.3 of Chapter 3 of this thesis, with $m_{a}=0, n=1$, and not multiplying by $100 \%$.

$$
\bar{Q}=\left[T_{1}\right]^{-1}[Q]\left[T_{2}\right]
$$

$\left[T_{1}\right]$ is the transformation for stress

$\left[T_{2}\right]$ is the transformation for strain

However, it is known that:

$$
\begin{aligned}
& \bar{Q}_{11}=Q_{11} m^{4}+2\left(Q_{12}+2 Q_{66}\right) m^{2} n^{2}+Q_{22} n^{4} \\
& \bar{Q}_{12}=\left(Q_{11}+Q_{22}-4 Q_{66}\right) m^{2} n^{2}+Q_{12}\left(n^{4}+m^{4}\right) \\
& \bar{Q}_{22}=Q_{11} n^{4}+2\left(Q_{12}+2 Q_{66}\right) m^{2} n^{2}+Q_{22} m^{4} \\
& \bar{Q}_{16}=\left(Q_{11}-Q_{12}-2 Q_{66}\right) m^{3} n+\left(Q_{12}-Q_{22}+2 Q_{66}\right) n^{3} m \\
& \bar{Q}_{26}=\left(Q_{11}-Q_{12}-2 Q_{66}\right) m n^{3}+\left(Q_{12}-Q_{22}+2 Q_{66}\right) n m^{3} \\
& \bar{Q}_{66}=\left(Q_{11}+Q_{22}-2 Q_{12}-2 Q_{66}\right) m^{2} n^{2}+Q_{66}\left(n^{4}+m^{4}\right)
\end{aligned}
$$

Here,

$$
\begin{aligned}
& m=\cos \theta \\
& n=\sin \theta \\
& {[\bar{Q}]=\left[\begin{array}{lll}
\bar{Q}_{11} & \bar{Q}_{12} & \bar{Q}_{16} \\
\bar{Q}_{12} & \bar{Q}_{22} & \bar{Q}_{26} \\
\bar{Q}_{16} & \bar{Q}_{26} & \bar{Q}_{66}
\end{array}\right]}
\end{aligned}
$$

In principal material coordinates, $Q_{16}=Q_{26}=0$. 


$$
\begin{aligned}
Q_{11} & =\frac{E_{1}}{1-v_{12} v_{21}} \\
Q_{12} & =\frac{v_{12} E_{2}}{1-v_{12} v_{21}}=\frac{v_{21} E_{1}}{1-v_{12} v_{21}} \\
Q_{22} & =\frac{E_{2}}{1-v_{12} v_{21}} \\
Q_{66} & =G_{12}
\end{aligned}
$$

Rule of Mixture

$$
\begin{aligned}
& E_{1}^{*}=E_{f} \cdot V_{f}+E_{m} \cdot V_{m} \\
& \text { But } \\
& V_{f}+V_{m}=1 \\
& E_{2}^{*}=\frac{E_{m}}{\left[V_{f}\left(\frac{E_{m}}{E_{f}}-1\right)+1\right]} \\
& \frac{1}{G_{12}^{*}}=\frac{V_{f}}{G_{f}}+\frac{V_{m}}{G_{m}} \\
& v_{12}^{*}=V_{f}\left(v_{f}-v_{m}\right)+E_{m} \cdot v_{m} \\
& v_{21}^{*}=\frac{E_{2}^{*}}{E_{1}^{*}} v_{12}^{*}
\end{aligned}
$$

For this study, the carbon fibre and the epoxy resin usually have the following properties:

Fibre:

Elastic Modulus $11\left(E_{f}\right): \quad$ E11 $=\quad 240000 \mathrm{MPa}$

Resin:

Elastic Modulus $\left(E_{m}\right): \quad \quad E=\quad 3070 \mathrm{MPa}$ 